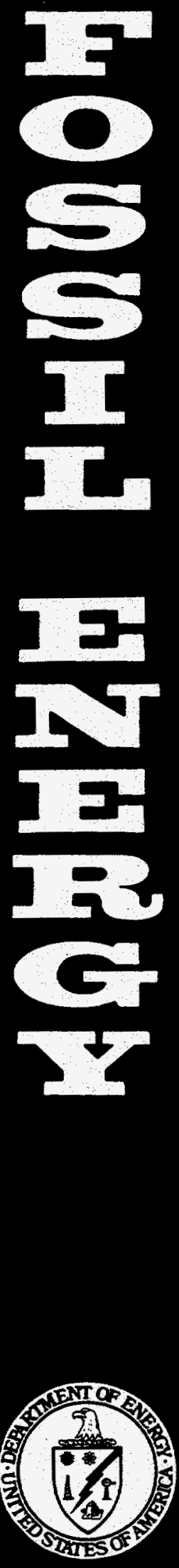

$$
\text { DOE/PC/ } / 91008 \text { - TI }
$$

CHARACTERIZATION OF FRACTURE RESERVOIRS USING STATIC AND DYNAMIC DATA: FROM SONIC AND 3D SEISMIC TO PERMEABILITY DISTRIBUTION

Annual Report

By

Jorge O. Parra, Ph.D.

Hughbert A. Collier, Ph.D.

Akhil Datta-Gupta, Ph.D.

Thomas E. Owen, Ph.D.

RECEIVED

JUL $1^{4} 1997$

0. 8.1

Sriram Peddibhotla

Pei-cheng Xu, Ph.D.

Brian J. Zook, Ph.D.

June 1997

PC

Performed Under Contract No. DE-AC22-94DC91008

Subcontract No. G4551 732

Southwest Research Institute

Instrumentation and Space Research Division

National Petroleum Technology Office

U. S. DEPARTMENT OF ENERGY

Tulsa, Oklahoma

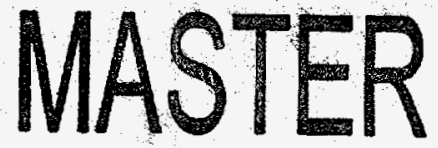

OSTRIBUTION OF THIS DOCUMENT IS UNLIMITED 


\section{DISCLAIMER}

This report was prepared as an account of work sponsored by an agency of the United States Government. Neither the United States Govemment nor any agency thereof, nor any of their employees, makes any warranty, expressed or implied, or assumes any legal liability or responsibility for the accuracy, completeness, or usefulness of any information, apparatus, product, or process disclosed, or represents that its use would not infringe privately owned rights. Reference herein to any specific commercial product, process, or service by trade name, trademark, manufacturer, or otherwise does not necessiarily constitute or imply its endorsement, recommendation, or favoring by the United States Government or any agency thereof. The views and opinions of authors expressed herein do not necessarily state or reflect those of the United States Government.

This report has been reproduced directly from the best available copy.

Available to DOE and DOE contractors from the Office of Scientific and Technical Information, P.O. Box 62, Oak Ridge, TN 37831; prices available from (615) 5768401.

Available to the public from the National Technical Information Service, U.S. Department of Commerce, 5285 Port Royal Rd., Springfield VA 22161 


$$
\text { DoE/PC/91008- Distribution Category }
$$

Characterization Of Fracture Reservoirs Using Static And Dynamic Data:

From Sonic And 3D Seismic To Permeability Distribution

Annual Report

By

Jorge O. Parra, Ph.D.

Hughbert A. Collier, Ph.D.

Akhil Datta-Gupta, Ph.D.

Thomas E. Owen, Ph.D.

Sriram Peddibhotla

Pei-cheng Xu, Ph.D.

Brain J. Zook, Ph.D.

June 1997

Work Performed Under Contract No. DE-AC22-94DC91008

Subcontract No. G4551 732

\author{
Prepared for \\ U.S. Department of Energy \\ Assistant Secretary for Fossil Energy \\ Robert E. Lemmon, Project Manager \\ National Petroleum Technology Office \\ P.O. Box 3628 \\ Tulsa, OK 74101
}

Prepared by:

Southwest Research Institute

Instrumentation and Space Research Division

DETRIBUTION OF THIS DOCUMENT IS UNLIMTED 


\section{DISCLAMIER}

Portions of this document may be illegible in electronic image products. Images are produced from the best available original document. 


\section{FOREWORD AND ACKNOWLEDGMENTS}

The work reported herein represents the first year work on the characterization of fracture reservoirs using static and dynamic data from sonic and 3D seismic to permeability distribution. The project coordination and supervision as well as the geophysical studies were performed by Dr. Jorge Parra from the Department of Electronic Systems, Instrumentation and Space Research Division, Southwest Research Institute. The petrophysical studies and the Technology Transfer plan were conducted by Dr. Hughbert Collier and Dr. Thomas Owen, respectively, both consultants to SwRI. The petroleum engineering work was performed by Dr. Akhil Datta-Gupta and Mr. Sriram Peddibhotla under a subcontract agreement between Southwest Research Institute and the University of Texas A\&M. Software development and computer assistance throughout the project were provided by Dr. Pei-cheng Xu and Dr. Brian Zook from Southwest Research Institute.

The assistance of Mr. Min Tham, project manager from BDM-Oklahoma is gratefully acknowledged. We thank Union Pacific Resources Company, in particular, Mr. James Peters, for his contribution of Twin Creek Reservoir data.

This report contains seven sections. Some individual sections contain their own list of references as well as appendices and conclusions when appropriate. The first section includes the introduction and a summary of the first-year project efforts. The next five sections describe the results of the project tasks: (1) Analysis of plane-harmonic waves in poroelastic and anisotropic layered media; (2) Analysis of an acoustic logging system in a fluid-filled borehole surrounded by an anisotropic poroelastic formation; (3) Semi-analytic approach to multiphase flow calculations; (4) Data collection and review, analysis and interpretation of existing data, and selection of study area; and (5) technology transfer. The last section includes general conclusions and accomplishments. 


\section{TABLE OF CONTENTS}

\section{$\underline{\text { Page }}$}

FOREWORD AND ACKNOWLEDGMENTS $\ldots \ldots \ldots \ldots \ldots \ldots \ldots \ldots \ldots$

I. INTRODUCTION AND SUMMARY OF PROJECT $\ldots \ldots \ldots \ldots \ldots \ldots \ldots$

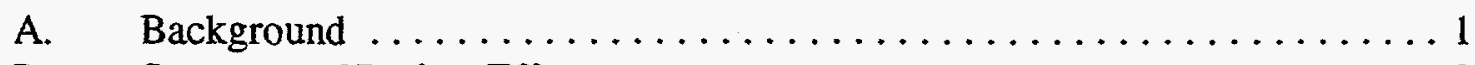

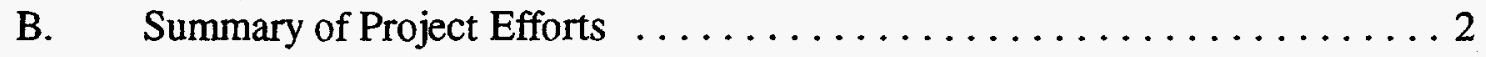

II. ANALYSIS OF PLANE-HARMONIC WAVES IN POROELASTIC AND ANISOTROPIC LAYERED MEDIA . . . . . . . . . . . . 4

A. The transversely isotropic poroelastic wave equation using Biot and squirt mechanism . . . . . . . . . . . . . . . . . . 4

B. Analysis of a layered transversely isotropic poroelastic medium (LTIPM) to incident plane waves . . . . . . . . . . . . . 31

C. Responses of seismic wave propagation in poroelastic media having azimuthal anisotropy $\ldots \ldots \ldots \ldots \ldots \ldots \ldots \ldots \ldots$

III. ANALYSIS OF AN ACOUSTIC LOGGING SYSTEM IN A FLUID-FILLED BOREHOLE SURROUNDED BY AN ANISOTROPIC POROELASTIC FORMATION

A. The response of an acoustic multipole in a fluid-filled borehole surrounded by a poroelastic formation $\ldots \ldots \ldots \ldots \ldots \ldots 76$

B. Acoustic waves in fluid-filled borehole surrounded by transversely isotropic formation with a horizontal axis of symmetry

IV. SEMIANALYTICAL APPROACH TO MULTIPHASE FLOW CALCULATIONS

A. Multiphase Streamline Modeling in Three Dimensions . . . . . . 107

B. Application of the theory to the La Cira field in Colombia . . . . . . . . . 124

V. SEISMIC SIGNATURES OF THE FRACTURED TWIN CREEK RESERVOIR, LODGEPOLE FIELD, UTAH-WYOMING OVERTHRUST BELT . . . . . . . . 129

A. Introduction . . . . . . . . . . . . . . . . . . . . . . 129

B. Geology . . . . . . . . . . . . . . . . . . . . . . . 129

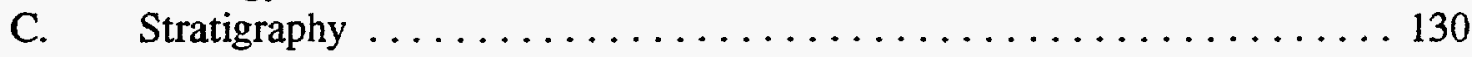

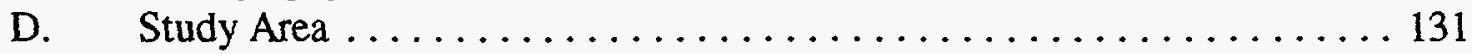

E. Geologic Controls on Fracture Distribution $\ldots \ldots \ldots \ldots \ldots \ldots \ldots$

F. Geologic Controls on Hydrocarbon Production ............. 134 


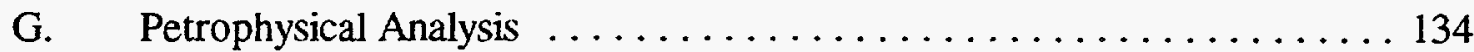

H. Integration of Surface Seismic and Well Log Data $\ldots \ldots \ldots \ldots \ldots 135$

VI. TECHNOLOGY TRANSFER $\ldots \ldots \ldots \ldots \ldots \ldots \ldots \ldots \ldots \ldots \ldots \ldots \ldots$

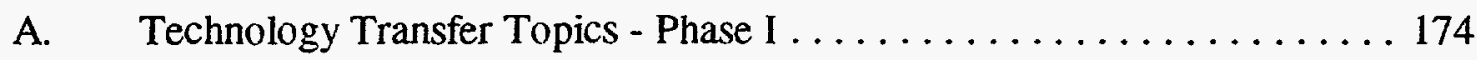

B. Industry Contacts and Application Interests $\ldots \ldots \ldots \ldots \ldots \ldots \ldots \ldots$

C. Project Newsletter .......................... 175

VII. CONCLUSIONS AND ACCOMPLISHMENTS $\ldots \ldots \ldots \ldots \ldots \ldots \ldots$ 


\section{A. Background}

In low porosity, low permeability zones, natural fractures are the primary source of permeability which affect both production and injection of fluids. The open fractures do not contribute much to porosity, but they provide an increased drainage network to any porosity. They also may connect the borehole to remote zones of better reservoir characteristics. An important approach to characterizing the fracture orientation and fracture permeability of reservoir formations is one based on the effects of such conditions on the propagation of acoustic and seismic waves in the rock.

The project is a study directed toward the evaluation of acoustic logging and 3Dseismic measurement techniques as well as fluid flow and transport methods for mapping permeability anisotropy and other petrophysical parameters for the understanding of the reservoir fracture systems and associated fluid dynamics. The principal application of these measurement techniques and methods is to identify and investigate the propagation characteristics of acoustic and seismic waves in the Twin Creek hydrocarbon reservoir owned by Union Pacific Resources (UPR) and to characterize the fracture permeability distribution using production data. This site is located in the overthrust area of Utah and Wyoming. UPR drilled six horizontal wells, and presently UPR has two rigs running with many established drill hole locations. In addition, there are numerous vertical wells that exist in the area as well as 3D seismic surveys. Each horizontal well contains full FMS logs and MWD logs, gamma logs, etc.

This 3-year research program combines advanced theoretical and numerical model studies with a balanced petrophysical and engineering program. Its aim is the development of advanced concepts of borehole seismic, surface seismic, and fluid flow dynamic methods that relate permeability anisotropy to acoustic and seismic signatures. Then one could understand the reservoir fracture system and to predict the permeability distribution throughout heterogeneous reservoirs using multiphase production data.

The topics of research for the first year of this project involve petrophysical analysis, and basic theoretical analysis in geophysics and petroleum engineering. Geophysical techniques include the analysis of plane-harmonic seismic waves in poroelastic and anisotropic layered media, as well as the theoretical development of an acoustic logging system in a fluid-filled borehole surrounded by an isotropic poroelastic formation and an anisotropic viscoelastic formation. Petroleum engineering techniques include the development of a semi-analytic approach to multiphase flow calculations and applications. The petrophysical analysis consists of evaluating the Twin Creek fractured reservoir in the overthrust area of Utah and Wyoming to characterize fractures and rock physical properties that will be used for the validation of theoretical advanced concepts developed in this project. 


\section{B. Summary of Project Efforts}

The first topic is devoted to the development of an analytical solution for planeharmonic seismic waves propagating in a poroelastic anisotropic media, including the Biot and squirt flow mechanisms. The solution was extended to simulate responses of a horizontally layered poroelastic medium including the second-rank tensor permeability and the second-rank tensor of the squirt-flow mechanism to simulate azimuthal anisotropy. After the theoretical work was completed, we developed software to calculate fluid pressure, the vector wavefield, and the displacement of the fluid relative to the solid. This software was used to conduct a parametric study of the angle of incidence, permeability anisotropy, and frequency content, allowing us to relate the permeability anisotropy to the dispersion and attenuation of seismic signatures. The second topic includes the development of an acoustic logging system in a fluid-filled borehole surrounded by an isotropic poroelastic medium. In addition, we developed the theoretical analysis of a multipole-acoustic source in a fluid-filled borehole surrounded by an anisotropic medium having axis of symmetry in the horizontal direction. In this case we implemented the boundary integral equation method of solution to calculate the acoustic logging response for a borehole surrounded by formations having azimuthal anisotropy.

The following topic is devoted to the development and testing of a three dimensional streamline simulator for modeling multiphase flow and transport in heterogeneous permeable media. The emphasis was on speed and accuracy so that the multiphase model can be embedded in an inversion scheme to derive fracture characteristics using production data. The streamline model was generalized to account for infill drilling, non-uniform initial conditions and mixed boundary conditions so that the model could be applied under a wide variety of field conditions. High resolution total variation diminishing schemes were used to solve the multiphase flow equations along streamlines to prevent numerical artifacts such as artificial dispersion. Novel mapping algorithms were used to account for changing well configurations during streamline simulation. The results from the streamline model were validated against commercial numerical simulators. The results indicate increased accuracy and significant savings in computation time.

The next topic discusses the integration of petrophysical data and 2D seismic data based on the data catalog of the Twin Creek reservoir owned by Union Pacific Resources in the Utah-Wyoming Overthrust belt. Several geological cross-sections were constructed and integrated with the migrated seismic data, the velocity inversion, and FMS data recorded in horizontal wells. The data integration with synthetics derived from well log information delineated the major geological units of interest in the Twin Creek reservoir. In addition the fractured density determined from an horizontal well correlates with a velocity anomaly observed in the migrated seismic data. In addition, the result of the integration has provided a twelve-layer model, including the fracture zones of interest containing the petrophysical information for each geological unit in the reservoir. This model was used to calculate synthetic seismic signatures for planning cross-well seismic surveys between wells spaced $2400 \mathrm{ft}$ apart at the Lodgepole field in the Twin Creek formation.

The last topic describes the initial efforts for transferring the concepts and results of the project to the oil and gas industry. Several contacts were made with interested companies engaged in oil and gas production to determine their specific interest and needs with respect to fractured 
reservoirs. Letters describing the technical objectives, the goals, and activities of this research project were distributed to each company. In fact, the project announcement letter and reply forms were sent to more than 100 petroleum geologists, geophysicists, and engineers in 26 oil companies. This announcement was well received by the industry as indicated by 60 percent positive return of replies expressing interest in receiving future project information and identifying their professional and technical expertise. 


\section{ANALYSIS OF PLANE-HARMONIC WAVES IN POROELASTIC AND ANISOTROPIC LAYERED MEDIA}

\section{A. The Transversely Isotropic Poroelastic Wave Equation Using Biot and Squirt Mechanisms}

The transversely isotropic poroelastic wave equation can be formulated to include the Biot and the squirt-flow mechanisms to yield a new analytical solution in terms of the elements of the squirt-flow tensor. The new model gives estimates of the vertical and the horizontal permeabilities as well as other measurable rock and fluid properties. In particular, the model estimates phase velocity and attenuation of waves traveling at different angles of incidence with respect to the principal axis of anisotropy. The attenuation and dispersion of the fast quasi-P wave and the quasi-SV wave are related to the vertical and the horizontal permeabilities. Modeling suggests that the attenuation of both the quasi-P wave and quasi-SV wave depend on the direction of permeability. For frequencies from 500 to $4500 \mathrm{~Hz}$, the quasi-P wave attenuation will be maximum in the direction of maximum permeability. To test the theory, interwell seismic waveforms, well logs, and hydraulic conductivity measurements (recorded in the fluvial Gypsy sandstone reservoir in Oklahoma) provide the material and fluid property parameters. For example, the analysis of petrophysical data suggests that the vertical permeability ( $1 \mathrm{md}$ ) is affected by the presence of mudstone and siltstone bodies, which are barriers to vertical fluid movement, and the horizontal permeability $(1640 \mathrm{md})$ is controlled by crossbedded and planar-laminated sandstones. The theoretical dispersion curves (based on measurable rock and fluid properties) and the phase velocity curve (obtained from seismic signatures) give the ingredients to evaluate the model. Theoretical predictions show the influence of the permeability anisotropy on the dispersion of seismic waves. These dispersion values derived from interwell seismic signatures are consistent with the theoretical model and with the direction of propagation of the seismic waves that travel parallel to the maximum permeability. This analysis with the new analytical solution is the first step toward a quantitative evaluation of the preferential directions of fluid flow in reservoir formation containing hydrocarbons. The results of the present work may lead to the development of algorithms to extract the permeability anisotropy from attenuation and dispersion data (derived from sonic logs and crosswell seismics) to map the fluid flow distribution in a reservoir.

The estimates of attenuation and dispersion of seismic waves are important for predicting the presence of fluids in a reservoir. Two important characteristics of reservoir rocks are porosity and permeability. Porosity can be determined from cores and well logs. Permeability can be measured on cores but is not measured directly from well logs. Permeability may differ from zone to zone, and it may vary directionally within a reservoir .

Although the in-situ permeability is considered a material property parameter difficult to predict, several researchers have developed methods for indirectly predicting permeability using full waveform sonic logs. Tang and Chelini (1993) developed high-resolution processing techniques using a simplified model to perform inversion of borehole Stonely waveform data to map the isotropic formation permeability. Schmitt (1989) addressed the attenuation and dispersion of the quasi P-waves and the quasi SV-wave in terms of a directional permeability, using the Biot and homogenization theories. 
It is well known that the Biot theory does not adequately describe the wave attenuation and dispersion in some reservoir rock in the range of frequency used on surface and crosswell seismic surveys. A different theory developed by O'Connell and Budiansky (1977) assumes that the passing wave in reservoir rock may cause pore fluid flow at a pore scale that they called "local flow" or squirt flow. This theory can adequately explain the large velocity dispersion in some rocks where the Biot theory fails. In fact, Dvorkin and Nur (1993) have shown that the squirt-flow mechanism results in much higher and realistic attenuation in saturated rocks than that predicted by the Biot mechanism.

A consistent theory dealing simultaneously with Biot and squirt-flow mechanisms has been presented by Dvorkin and Nur (1993). The theory includes those mechanisms by considering the fluid motion parallel (the Biot mechanism) and transverse (the squirt-flow mechanism) to the direction of a planar P-wave. The fluid is free to move not only parallel but also perpendicular to the direction of wave propagation. The Biot-squirt flow model relates the dynamic poroelastic behavior of a saturated rock to the poroelastic parameters, porosity, permeability, fluid compressibility and viscosity, and the characteristic squirt-flow length.

In this paper, the general theory of propagation of elastic waves in fluid-saturated porous media, based on the concept of a two-phase medium given by Biot(1962a), Schmitt( 1989), and Parra and Xu (1994), provides a framework to analyze and interpret the fluid flow mechanisms. The constitutive equations for anisotropic porous media originally given by Biot (1955) and Biot (1962a) have been formulated by Kazi-Azoual, et al., (1988) in accordance with the homogenization theory (Auriault, et al., 1985). The result of Auriault's work has lead to a macroscopic dynamics similar to that of Biot theory. In particular, the complex frequency-dependent permeability, namely, the generalized Darcy coefficient, is introduced by the homogenization theory. The formulation of the transversely isotropic wave equation including the Biot and squirt mechanisms is based on the constitutive equations given by Biot's theory and the homogenization theory. The latter theory provides the second-rank complex tensor permeability; the squirt-flow tensor is introduced in the constitutive stress equation associated with the pore fluid to yield a new partial differential equation that relates the fluid pressure to the dilatation of the rock matrix. Following this analysis, we formulated and derived the poroelastic wave equation including the Biot and the squirt mechanisms to obtain a dispersion equation of third degree. Its solution gives wavenumbers from which the phase velocity and attenuation of the quasi-P waves and the quasi-SV wave are determined. Numerical models are given to relate the directional attenuation and dispersion with anisotropy permeability.

To test the model, a field example provides the phase velocity for waves traveling between wells in the Gypsy sandstone reservoir in Oklahoma. This example illustrates the applicability of the solution derived in this paper as a potential modeling approach for solving practical reservoir characterization problems.

\section{The Poroelastic Wave Equation Including the Biot and the Squirt Mechanisms}

The formulation of the transversely isotropic poroelastic wave equation including the Biot and the squirt-flow mechanisms is based on the constitutive relations (i.e., the total stress tensor 
of the anisotropic porous medium, and the stress tensor in the pore fluid), the momentum balance equation for total stress, and the generalized Darcy's law, in the framework of Biot's theory. These equations in the frequency domain (assuming $\exp (-j \omega t)$ variation) are:

$$
\begin{aligned}
\underset{\sim}{\sigma} & =\underset{\sim}{\boldsymbol{\tau}} \cdot \underset{\sim}{\boldsymbol{e}}-\underset{\sim}{\alpha} p \\
\sigma_{i j}^{(f)} & =-\phi p \delta_{i j}=\frac{\phi}{\beta}[\underset{\sim}{\alpha} \cdot \underset{\sim}{\boldsymbol{e}}+\phi \nabla \cdot(U-\boldsymbol{u})] \delta_{i j}, \\
\frac{\partial \sigma_{i j}}{\partial x_{j}} & =\nabla \cdot \underset{\sim}{\sigma}=-\omega^{2}\left[\rho_{s}(1-\phi) \boldsymbol{u}+\phi \rho_{f} U\right] \\
\boldsymbol{w} & =\phi(U-\boldsymbol{u})=-\underset{\sim}{\boldsymbol{K}}(\omega)\left(\omega^{2} \rho \boldsymbol{\mu}-\nabla p\right) / j \omega .
\end{aligned}
$$

In these equations, $\mathbf{e}$ is the strain tensor of the porous medium; $\mathrm{p}$ is the fluid pressure; $\phi$ is the porosity; $u$ and $U$ are the particle displacement of the solid and the fluid, respectively, and $\rho_{\mathrm{s}}$ and $\rho_{\mathrm{f}}$ are the solid and fluid densities. In addition, $\tau$ is the solid-frame stiffness tensor containing five independent drained elastic coefficients (i.e., $c_{11}, c_{12}, c_{13}, c_{44}$, and $c_{33}$ ). The poroelastic coefficients of the effective stress of the second-rank tensor $\underset{\sim}{\alpha}$ (which are obtained at zero pore pressure) are given

$$
\alpha_{1}=1-\left(c_{11}+c_{12}+c_{13}\right) / 3 K_{s} \text {, }
$$

and

$$
\alpha_{3}=1-\left(2 c_{13}+c_{33}\right) / 3 K_{s}
$$

where $K_{s}$ is the bulk modulus of the grains. On the other hand, $\beta$ is the compressibility coefficient determined under undrained conditions, given by

$$
\beta=\phi / K_{f}+(1-\phi) / K_{s}-\left[2\left(c_{11}+c_{12}+2 c_{13}\right)+c_{33}\right] / 9 K_{s}^{2},
$$

where $K_{f}$ is the fluid bulk modulus. In the last equation (4), $w$ is the displacement of the fluid relative to solid, and $\mathbf{K}(\omega)$ is the second-rank complex tensor frequency-dependent permeability described by two permeability constants. After Biot (1956), Biot (1962a) and Schmitt (1989), the complex permeability elements for a low-frequency range are given by

$$
\mathrm{K}_{\mathrm{l}}(\omega)=\frac{\mathrm{j} \phi}{\omega \rho_{\mathrm{f}}}\left[\frac{\rho_{\mathrm{a}} / \rho_{\mathrm{f}}+\phi}{\phi}+\frac{\mathrm{j} \omega_{\mathrm{l}}}{\omega}\right]^{-1},
$$

where $\frac{\omega \ell}{\omega}=\frac{\eta \phi}{\tilde{k}_{\ell} \rho_{f} \omega}, \ell=1$ and 3 , and $\rho_{\mathrm{a}}$ is the additional density caused by the fluid (Biot, 1956). 
The parameter $\eta$ is the viscosity of the saturating fluid, and $\tilde{\mathrm{k}}_{\ell}$ is the intrinsic permeability along the principal direction $\ell$.

The squirt-flow mechanism is incorporated in the constitutive equation (2) by assuming a poroelastic medium axially symmetric about the $\mathrm{z}$-axis and stating that the permeability tensor and the porous matrix have the same plane propagation direction. In this case we define the squirt-flow tensor as

$$
\underset{\sim}{S}(\omega)=\left(\begin{array}{ccc}
s_{3}(\omega) & 0 & 0 \\
0 & s_{3}(\omega) & 0 \\
0 & 0 & s_{1}(\omega)
\end{array}\right) .
$$

To derive the elements of the squirt-flow tensor, we apply the method given in Dvorkin and Nur (1993) by assuming a cylindrical volume of rock geometries in the $\mathrm{x}, \mathrm{y}$, and $\mathrm{z}$ directions. For example, a sideways flow in the xy plane can be represented by a cylinder with its axis parallel to the wave motion in the $\mathrm{z}$-direction. The radius of this cylinder is equal to the average squirt-flow length $R_{1}$ which is associated with the horizontal permeability $\tilde{k}_{x}$ in the xy plane. In a similar manner, a vertical flow in the yz plane can be represented by a cylinder having its axis parallel to the wave motion in the $\mathrm{x}$-direction. In this case, the radius of the cylinder is equal to average squirt-flow length $\mathrm{R}_{3}$ associated with the vertical permeability $\tilde{\mathrm{k}}_{\mathrm{z}}$. Thus, the elements of the squirt-flow tensor are given

$$
s_{l}=1-\frac{2 J_{1}\left(\gamma_{l} R_{l}\right)}{\gamma_{l} R_{l} J_{0}\left(\gamma_{l} R_{l}\right)}
$$

where

$$
\gamma_{l}^{2}=\frac{\rho_{\mathrm{f}} \omega^{2}}{(\phi / \beta)}\left[\frac{\rho_{\mathrm{a}} / \rho_{\mathrm{f}}+\phi}{\phi}+\frac{j \omega_{l}}{\omega}\right]
$$

and

$$
\frac{\omega_{\ell}}{\omega}=\frac{\eta \phi}{\tilde{\mathrm{k}}_{\ell} \rho_{\mathrm{f}} \omega} \text {; for } \ell=1 \text { and } 3 \text {. }
$$

The total pressure for a 3-D wave motion including the Biot and the squirt-flow mechanisms in a transversely isotropic medium can be written as

$$
\mathrm{p}=-\frac{\phi}{\beta} s_{1}\left[\frac{\partial \mathrm{U}_{\mathrm{z}}}{\partial \mathrm{z}}+\frac{\left(\alpha_{3}-\phi\right)}{\phi} \frac{\partial \mathrm{u}_{\mathrm{z}}}{\partial \mathrm{z}}\right]-\frac{\phi}{\beta} s_{3}\left[\frac{\partial \mathrm{U}_{\mathrm{y}}}{\partial \mathrm{y}}+\frac{\left(\alpha_{1}-\phi\right)}{\phi} \frac{\partial \mathrm{u}_{\mathrm{y}}}{\partial \mathrm{y}}\right]-\frac{\phi}{\beta} s_{3}\left[\frac{\partial \mathrm{U}_{\mathrm{x}}}{\partial \mathrm{x}}+\frac{\left(\alpha_{1}-\phi\right)}{\phi} \frac{\partial \mathrm{u}_{\mathrm{x}}}{\partial \mathrm{x}}\right],
$$

where $\phi / \beta=1 /\left\{1 / \mathrm{K}_{\mathrm{f}}+\left[\left(2 \alpha_{1}+\alpha_{3}\right) / 3-\phi\right] / \mathrm{K}_{\mathrm{s}}\right\}$. This equation was determined using equations (5a), (5b), and (6). The first term in brackets of equation (10) represents the contribution to the total pressure of the squirt-flow mechanism associated with particle motion in the $\mathrm{z}$-direction. The last two terms represent the contribution to the fluid pressure of the squirt-flow mechanism associated with wave motion in $\mathrm{x}$ - and $\mathrm{y}$-directions. To give a more general physical interpretation 
to the fluid pressure and for further theoretical analysis, we express equation (10) in terms of the displacement of the fluid relative to the solid given by $\mathbf{w}=\phi(\mathbf{U}-\mathbf{u})$ to yield

$$
\mathbf{p}=-\frac{1}{\beta}\left[s_{3} \nabla \cdot \mathbf{w}+\left(s_{1}-s_{3}\right) \frac{\partial w_{z}}{\partial z}\right]-\frac{1}{\beta}\left[s_{3} \alpha_{1} \nabla \cdot \mathbf{u}+\left(s_{1} \alpha_{3}-s_{3} \alpha_{1}\right) \frac{\partial u_{z}}{\partial z}\right] \text {. }
$$

This equation is the modified constitutive equation (2); it describes the Biot and the squirt-flow mechanisms for transversely isotropic conditions. The first term in brackets represents the pressure associated with the fluid-flow relative to the solid and is mainly controlled by the Biot and the squirtflow mechanisms. The second term represents the pressure associated with the displacement of the porous matrix; the response is controlled by the squirt-flow mechanism coupled to the stiffness constants of the rock matrix. The special case of waves traveling in a poroelastic isotropic medium characterized by the Biot and the squirt-flow mechanisms can be derived by making $\alpha_{1}=\alpha_{3}=\alpha$ and $s_{1}=s_{3}=s$ in equations (10) and (11). These equations are reduced to

$$
\mathrm{p}=-\frac{\phi}{\bar{\beta}} s\left(\nabla \cdot \mathbf{U}+\frac{(\alpha-\phi)}{\phi} \nabla \cdot \mathbf{u}\right)
$$

and

$$
\mathrm{p}=-\frac{1}{\bar{\beta}} \mathrm{s}(\nabla \cdot \mathbf{w}+\alpha \nabla \cdot \mathbf{u})
$$

where the ratio $\phi / \beta$ becomes $\phi / \bar{\beta}=1 /\left[1 / \mathrm{K}_{\mathrm{f}}+(\alpha-\phi) / \phi \mathrm{K}_{\mathrm{s}}\right]$. This factor multiplied by s yields the coefficient $F_{s q}$ given in Dvorkin and Nur (1993). Equation (12a) corresponds to the constitutive equation (2) for isotropic conditions, which has been modified by introducing the squirt-flow mechanism. In equations (12), $\alpha$ is the poroelastic coefficient of effective stress: $\alpha=1-\mathrm{K} / \mathrm{K}_{\mathrm{s}}$, where $\mathrm{K}$ is the bulk modulus of the drained porous matrix.

A new partial differential equation for the fluid pressure and the displacement vector of the solid is constructed using the generalized Darcy's equation (4) and equation (11). Thus, after some algebraic development, this new coupled wave equation in terms of the Biot and the squirt-flow mechanisms is

$$
s_{3} \theta_{1} \nabla^{2} p-\beta p+\left(\theta_{3} s_{1}-s_{3} \theta_{1}\right) \frac{\partial^{2} p}{\partial z^{2}}-s_{3} \hat{\alpha}_{1} \nabla \cdot u-\left(s_{1} \dot{\alpha}_{3}-s_{3} \hat{\alpha}_{1}\right) \frac{\partial u_{z}}{\partial z}=0
$$

This equation for isotropic conditions and no squirt-flow mechanism, i.e. $\theta_{1}=\theta_{3}=\theta, s_{1}=s_{3}=1$ and $z_{1}=z_{3}=\&$ is reduced to

$$
\theta \nabla^{2} \mathrm{p}-\bar{\beta} \mathrm{p}-\& \nabla \cdot \mathbf{u}=0
$$


where $\theta=-K(\omega) / j \omega$ and $\alpha=\alpha+\rho_{f} \omega^{2} \theta$. Equation (13) is the same equation given in Parra (1991) and Parra and $\mathrm{Xu}$ (1994).

The poroelastic system of differential equations including the Biot and the squirt mechanisms associated with the displacement components $u_{x} \equiv u_{1}, u_{y} \equiv u_{2}, u_{z} \equiv u_{3}$, and the pressure $\mathrm{p}$, is obtained by eliminating the displacement vector of the liquid $U$ and the total stress tensor of the porous medium $\sigma$, between the constitutive equations (1), (3), and (4) and equation (13). This system of equations is reduced to one set of three coupled partial differential equations associated with two quasi compressional waves $\mathrm{qP}_{\text {fast }}$ and $\mathrm{qP} \mathrm{P}_{\text {slow }}$ and a quasi-vertical polarized shear wave $\mathrm{qSV}$, and a separate differential equation associated with waves polarized in the horizontal plane (SH-waves). The poroelastic system of equations is defined by the displacement components $u_{x}$ and $u_{z}$ and the pressure p:

$$
\begin{array}{r}
{\left[c_{11} \frac{\partial^{2}}{\partial x^{2}}+c_{44} \frac{\partial^{2}}{\partial z^{2}}+\omega^{2} \beta_{x}\right] u_{x}+\left(c_{13}+c_{44}\right) \frac{\partial^{2}}{\partial x \partial z} u_{z}-\hat{\alpha}_{x} \frac{\partial p}{\partial x}=0} \\
\left(c_{13}+c_{44}\right) \frac{\partial^{2} u_{x}}{\partial x \partial z}+\left[c_{44} \frac{\partial^{2}}{\partial x^{2}}+c_{33} \frac{\partial^{2}}{\partial z^{2}}+\omega^{2} \hat{\rho}_{z}\right] u_{z}-\alpha_{z} \frac{\partial p}{\partial z}=0 \\
-s_{3} \varepsilon_{x} \frac{\partial u_{x}}{\partial x}-s_{1} \varepsilon_{z} \frac{\partial u_{z}}{\partial z}+\left(s_{3} \theta_{x} \frac{\partial^{2}}{\partial x^{2}}+s_{1} \theta_{z} \frac{\partial^{2}}{\partial z^{2}}-\beta\right) p=0,
\end{array}
$$

where:

$$
\begin{aligned}
\alpha_{\ell} & =\alpha_{\ell}+\omega^{2} \rho_{\mathrm{f}} \theta_{\ell}, \\
\beta_{\ell} & =\rho+\omega^{2} \rho_{\mathrm{f}}^{2} \theta_{\ell}, \\
\rho & =\rho_{s}(1-\phi)+\phi \rho_{\mathrm{f}},
\end{aligned}
$$

and $\quad \theta_{\ell}=\frac{-K_{l}(\omega)}{j \omega} ; \quad$ for $\ell=x$ and $z$.

The uncoupled partial differential equation is defined in terms of the displacement component $\mathrm{u}_{\mathrm{y}}$ :

$$
\left[c_{66} \frac{\partial^{2}}{\partial x^{2}}+c_{44} \frac{\partial^{2}}{\partial z^{2}}+\omega^{2} \beta_{x}\right] u_{y}=0 \text {. }
$$

\section{Plane Wave Solution of the Wave Equation Containing the Biot and the Squirt Mechanisms}

To solve the coupled system of equations (15) and the uncoupled partial differential equation (16), a plane harmonic wave propagating in the $(\mathrm{x}, \mathrm{z})$ plane is assumed to be represented by

$$
\left(u_{x}, u_{y}, u_{z}, p\right)=\left(b_{x}, b_{y}, b_{z}, P\right) \exp [j(k x+z \xi)]
$$


This expression for a plane wave propagating in an anisotropic medium is substituted in the system of equations (15) to yield

$$
D(\xi, k)=\left(\begin{array}{ccc}
c_{11} k^{2}+c_{44} \xi^{2}-\beta_{x} \omega^{2} & \left(c_{13}+c_{44}\right) \xi k & \alpha_{x} j k \\
\left(c_{13}+c_{44}\right) \xi k & \left(c_{44} k^{2}+c_{33} \xi^{2}-\beta_{z} \omega^{2}\right) & \alpha_{z} j \xi \\
s_{3} \alpha_{x} j k & s_{1} \alpha_{z} j \xi & \left(s_{3} \theta_{x} k^{2}+s_{1} \theta_{z} \xi^{2}+\beta\right)
\end{array}\right) .
$$

The wavenumber $\xi$, given by the determinant, $D(k, \xi)=0$, are solutions of the dispersion equation of third degree in $\xi^{2}$, which is written as

$$
\xi^{6}+\mathrm{P}_{4} \xi^{4}+\mathrm{P}_{2} \xi^{2}+\mathrm{P}_{0}=0
$$

where

$$
\begin{aligned}
& \mathrm{P}_{4}=\frac{\varepsilon_{\mathrm{z}}^{2}}{\theta_{\mathrm{z}} \mathrm{c}_{33}}+\mathrm{f}^{2}+\mathrm{v}^{2}+\chi^{2}+\mathrm{q}^{2}, \\
& \mathrm{P}_{2}=\varepsilon_{\mathrm{z}}^{2}\left(\mathrm{k}^{2} \epsilon^{2}-\frac{\omega^{2} \beta_{\mathrm{x}}}{\mathrm{c}_{44}}\right) / \theta_{\mathrm{z}} \mathrm{c}_{33}+\mathrm{p}^{2}+\mathrm{f}^{2}\left(v^{2}+\chi^{2}+\mathrm{q}^{2}\right),
\end{aligned}
$$

and $\quad P_{0}=\left(\frac{s_{3}}{s_{1}}\right) \varepsilon_{x}^{2} k^{2}\left(k^{2}-\omega^{2} \beta_{z} / c_{44}\right) / \theta_{z} c_{33}+f^{2} p^{2}$,

in which: $v^{2}=\mathbf{k}^{2}-\omega^{2} \tilde{\rho}_{\mathbf{z}} / \mathrm{c}_{33}$,

$$
\begin{aligned}
& \chi^{2}=\mathrm{k}^{2}-\omega^{2} \beta_{\mathrm{x}} / \mathrm{c}_{44}, \\
& \mathrm{f}^{2}=\left(\frac{\mathrm{s}_{3}}{\mathrm{~s}_{1}}\right) \frac{\theta_{\mathrm{x}}}{\theta_{\mathrm{z}}} \mathrm{k}^{2}+\frac{\beta}{\theta_{\mathrm{z}} \mathrm{s}_{1}},
\end{aligned}
$$

$\epsilon^{2}=c_{11} / c_{44}+c_{33}\left(\frac{s_{3}}{s_{1}}\right) \alpha_{x}^{2} / c_{44} \alpha_{z}^{2}-\alpha_{x}\left(1+\frac{s_{3}}{s_{1}}\right)\left(c_{13}+c_{44}\right) / \alpha_{z} c_{44}$

$$
q^{2}=k^{2}\left[c_{33}\left(c_{11}-2 c_{44}\right)-c_{13}\left(c_{13}+2 c_{44}\right)\right] / c_{33} c_{44}
$$

and

$$
\mathrm{p}^{2}=\left(c_{11} / \mathrm{c}_{33}\right)\left(\mathrm{k}^{2}-\beta_{\mathrm{z}} \omega^{2} / \mathrm{c}_{44}\right)\left(\mathrm{k}^{2}-\beta_{\mathrm{x}} \omega^{2} / \mathrm{c}_{11}\right)
$$

The solution of equation (19) gives three independent complex wavenumbers (i.e., $\xi_{\mathrm{j}}, \mathrm{i}=1,3$ ) associated with the quasi $P$-waves (fast and slow compressional waves) and the quasi SV-wave. These waves are called quasi waves because they approach the fast $\mathrm{P}$-wave, slow $\mathrm{P}$-wave, and the SV-wave as the degree of anisotropy of the permeability tensor, the squirt-flow tensor, and the 
porous matrix stiffness tensor vanish. The phase velocities and attenuation of the quasi compressional (fast and slow) and the quasi SV-waves can be obtained directly from the complex wavenumber roots $\left(\xi_{\mathrm{i}}, \mathrm{i}=1,3\right)$ as

$$
c_{i}(\omega)=\omega / \text { real }\left[\left(\xi_{j}^{2}+k^{2}\right)^{1 / 2}\right]
$$

and

$$
\mathrm{Q}_{\mathrm{i}}^{-1}=2 \mathrm{imag}\left[\left(\xi_{\mathrm{i}}^{2}+\mathrm{k}^{2}\right)^{1 / 2}\right] / \operatorname{real}\left[\left(\xi_{\mathrm{i}}^{2}+\mathrm{k}^{2}\right)^{1 / 2}\right],
$$

where $\mathrm{k}=\omega \sin (\delta) / \mathrm{v}$, in which $\mathrm{v}$ is the phase velocity of the plane wave traveling in the $(\mathrm{x}, \mathrm{z})$ plane making an angle $\delta$ with the $\mathrm{z}$ axis. On the other hand, for a horizontal polarized shear wave motion the propagation characteristics are obtained directly from the wavenumber given by

$$
\xi_{\mathrm{SH}}=\left[-\frac{c_{66}}{c_{44}} k^{2}+\frac{\omega^{2} \beta_{x}}{c_{44}}\right]^{1 / 2},
$$

where $\beta_{\mathrm{x}}=\rho_{\mathrm{s}}(1-\phi)+\rho_{\mathrm{f}} \phi+\omega^{2} \rho_{\mathrm{f}}^{2} \theta_{\mathrm{x}}$. That is, the attenuation and the phase velocity of the SH-wave depend on only the horizontal permeability.

As a check on the derivation of equation (19), phase velocities associated with wave propagation through an isotropic poroelastic medium can be obtained directly from equation (19). This can be done by replacing in the equations for the coefficients $\mathrm{P}_{0}, \mathrm{P}_{2}$, and $\mathrm{P}_{4}$,

$$
\begin{aligned}
& c_{13}=\lambda ; c_{44}=c_{66}=\mu ; c_{11}=c_{33}=\lambda+2 \mu ; \theta_{x}=\theta_{z}=\theta ; d_{x}=\alpha_{z}=\& ; \\
& \beta_{\mathrm{x}}=\beta_{\mathrm{z}}=\beta ; \tilde{\mathrm{k}}_{\mathrm{h}}=\tilde{\mathrm{k}}_{\mathrm{v}}=\hat{\mathrm{k}} ; \text { and } \mathrm{s}_{3}=\mathrm{s}_{1}=\mathrm{s} .
\end{aligned}
$$

In this case, $\lambda=(1-\phi) \bar{\lambda}$, and $\mu=(1-\phi) \bar{\mu}$, in which $\bar{\lambda}$ and $\bar{\mu}$ are the Lamé constants of the rock matrix. The substitution given in (22) yields the following third degree equation in $\xi^{2}$

$$
\left(\xi^{2}+\mathbf{k}^{2}-\mathbf{k}_{s}^{2}\right)\left(\xi^{2}+\mathbf{k}^{2}-\lambda_{1}^{2}\right)\left(\xi^{2}+\mathbf{k}^{2}-\lambda_{2}^{2}\right)=0,
$$

where $\lambda_{1}$ and $\lambda_{2}$ are the wavenumbers associated with fast and slow compressional waves traveling in a fluid-filled isotropic porous medium characterized by the Biot and the squirt mechanisms, and $\mathrm{k}_{s}$ is the wavenumber associated with the vertical polarized shear wave. These wave parameters are summarized as follows

$$
\begin{aligned}
& \lambda_{1}=[(\mathrm{b}+\Delta) / 2]^{1 / 2}, \\
& \lambda_{2}=[(\mathrm{b}-\Delta) / 2]^{1 / 2}, \\
& \mathrm{k}_{s}=\left[\omega^{2} \beta / \mu\right]^{1 / 2},
\end{aligned}
$$


where

$\begin{array}{lll}\Delta & =\left[\mathrm{b}^{2}+4 \mathrm{k}_{\mathrm{p}}^{2} \beta / \mathrm{s} \theta\right]^{1 / 2} \\ \text { and } \quad \mathrm{k}_{\mathrm{p}}^{2} & =\omega^{2} \beta /(\lambda+2 \mu) .\end{array}$

The squirt-flow parameter $s$ is given by equation (9) for $s_{1}=s_{2}=s$, and the parameters $\&$ and $\bar{\beta}$ are those recalled to describe equations (10) and (12). In this case, the phase velocities and attenuations of the compressional waves (fast and slow) and the shear wave are given by

and

$$
\begin{array}{lll}
\mathrm{v}_{\text {fast }} & =\omega / \text { real }\left(\lambda_{1}\right), \\
\mathrm{Q}_{\text {fast }}^{-1} & =2 \text { imag }\left(\lambda_{1}\right) / \text { real }\left(\lambda_{1}\right), \\
\mathrm{v}_{\text {slow }} & =\omega / \text { real }\left(\lambda_{2}\right), \\
\mathrm{Q}_{\text {slow }}^{-1} & =2 \text { imag }\left(\lambda_{2}\right) / \text { real }\left(\lambda_{2}\right), \\
\mathrm{v}_{\mathrm{sv}} & =\omega / \text { real }\left(\mathrm{k}_{\mathrm{s}}\right), \\
\mathrm{Q}_{\mathrm{sv}}^{-1} & =2 \text { imag }\left(\mathrm{k}_{\mathrm{s}}\right) / \text { real }\left(\mathrm{k}_{\mathrm{s}}\right) .
\end{array}
$$

The solution of the cubic dispersion equation (19) gives the wavenumbers, from which the phase velocity and attenuation of the quasi $P$-waves and the quasi $S V$-wave are determined to analyze a transversely isotropic medium characterized by its permeability tensor, its squirt-flow tensor and its porous matrix tensor. The elements of the squirt-flow tensor are coupled through the coefficients of the cubic equation, namely, $\mathrm{P}_{0}, \mathrm{P}_{2}$, and $\mathrm{P}_{4}$. This suggests that the phase velocity and attenuation of the three quasi waves will be influenced by the elements of the squirt-flow tensor. On the other hand, the wavenumbers associated with the poroelastic isotropic wave equation (23) show that the quasi-P waves depend of the squirt-flow element $s$ and the SV-wave is independent of $s$. In this case, the SV-wave depends on permeability only through the Biot mechanism.

\section{Extensions of the Present Solution and Potential Applications}

The wavenumbers determined from the solution of the cubic dispersion equation (19) can be used to construct expressions for the vector displacement components and the fluid pressure. This can be done by expressing these wavefields in terms of unknown wave coefficients multiplied by plane waves containing the quasi-wavenumbers. Such expressions will lead to the stressdisplacement matrix in which elements have the same characteristics as in a Green tensor. Once this is developed, seismic waveforms can be calculated to predict fluid-pressure seismic signatures as well as particle velocity (or particle displacement) signatures. In addition, this type of wave-field representation can be applied, for example, to develop the boundary value problems of a plane wave reflecting at a layer boundary (which separates two half-spaces of different anisotropy permeability), and a plane wave propagating in a multilayered medium.

An application could be to examine a poroelastic anisotropic formation in which fluid saturation and fluid contact boundaries (e.g., oil and water) are considered. The analysis can be aimed at evaluating how acoustic waves and their reflection coefficients at the boundaries are affected by the degree of anisotropy of both porous matrix and the complex tensor permeability (as a function of different angles of incidence between the direction of propagation and the principal axis of anisotropy) when the Biot and the squirt-flow mechanisms are considered. In this case, it is reasonable to expect that there can be fluid exchange motions across the oil and water interface when 
seismic wave motions are present. The interaction may affect the compressional waves and shear wave velocity and the amplitude of the waves. This basic theoretical treatment represents the first step toward a quantitative evaluation of similar effects in layered reservoir formations containing hydrocarbons.

In addition, the present theoretical analysis can be extended to treat the boundary value problem of a fluid-filled borehole surrounded by a poroelastic anisotropic formation which may be represented by fractures oriented perpendicular or parallel to the borehole. This solution can be used to produce full waveforms of wide-band multipole source excitations for acoustic signal frequencies capable of providing high resolution fracture reservoir structure. Stonely waves and flexure waves can be calculated when the squirt-flow mechanism is included in the solution to study the influence of the permeability anisotropy on the phase velocity and attenuation of acoustic logging signatures. This analysis may lead to the development of new algorithms to extract anisotropy permeability from dispersion and attenuation data derived from sonic logs (dipole and monopoles).

\section{Numerical Examples}

The main purpose of the present analytical study is to relate the tensor permeability to attenuation and dispersion of seismic waves for future developments of a Green's tensor to simulate seismic waves propagating in heterogeneous poroelastic media. The first step in the numerical calculations is to check the solution for the attenuation and dispersion equations given by equation (20), when the Biot and the squirt-flow mechanisms are included. In this case, we select the same material property and fluid property parameters used in Dvorkin and Nur (1993). These parameters together with $\mathrm{P}$-wave and S-wave velocities of the porous rock matrix are given in Table 1. At the same time as we check the solution, we analyze the effect of frequency on the attenuation and phase velocity curves using the horizontal permeabilities $\tilde{\mathrm{k}}_{\mathrm{h}}=1.25,2.5,3.75$ and 5 $\mathrm{md}$, and the vertical permeability of $\tilde{\mathrm{k}}_{\mathrm{v}}=1.25 \mathrm{md}$. Figure 1 shows that as the horizontal permeability decreases, the attenuation peak is shifted to the lower frequency. In addition, when $\tilde{\mathrm{k}}_{\mathrm{h}}=\tilde{\mathrm{k}}_{\mathrm{v}}=1.25$ $\mathrm{md}$, the attenuation and dispersion curves correspond to that of an isotropic poroelastic medium which is the same example given in Dvorkin and Nur (1993) for $\tilde{\mathrm{k}}=1.25 \mathrm{md}$. In general, both results are practically identical with the exception that Figure 1 shows the phase velocity and attenuation curves intercepting their corresponding vertical axis slightly higher than those intercepted in the Dvorkin and Nur example. Fortunately, the phase velocity at lower frequency can be checked directly using an equation given by Dvorkin and Nur (1993);

$$
\mathrm{V}_{\mathrm{po}}=\left[\mathrm{M} /\left((1-\phi) \rho_{\mathrm{s}}+\phi \rho_{\mathrm{f}}\right)\right]^{1 / 2}
$$

where $\mathrm{M}$ is the uniaxial modulus. Thus, substituting the corresponding physical property parameters given in Table 1 into equation (26) the resulting velocity $V_{p o}=3.84 \mathrm{~km} / \mathrm{s}$ is the value given in Figure 1 for $\mathrm{M}=35.48 \mathrm{GPa}$ and $\rho_{s}=2,650 \mathrm{~kg} / \mathrm{m}^{3}$.

In the next example, we analyze the effect of attenuation of the quasi $P$-wave and the quasi SV-wave on the directional permeability as a function of the angle of propagation with respect to the vertical, which varies from $0^{\circ}$ to $90^{\circ}$. The horizontal permeability is $1000 \mathrm{md}$ and vertical the 
permeability is $100 \mathrm{md}$. In Figure 2, the different curves correspond to values of the frequency that vary from $500 \mathrm{~Hz}$ to $4500 \mathrm{~Hz}$ by steps of $1000 \mathrm{~Hz}$. In Figure $2 \mathrm{a}$, the curves show that the quasi-P wave attenuation (fast wave) is maximum at all frequencies for the direction of propagation perpendicular to the vertical permeability $\tilde{\mathrm{k}}_{\mathrm{v}}=100 \mathrm{md}$. This suggests that the attenuation depends on the direction of the propagation; the quasi P-wave attenuation will be maximum in the direction of maximum permeability. Alternatively, the quasi SV-wave attenuation is maximum at all frequencies when the wave propagation is perpendicular to the direction of maximum permeability (see Figure 2b). In this case, the quasi-SV wave attenuation is controlled by the maximum permeability. These examples suggest that variations of attenuation of both the quasi $\mathrm{P}$-wave and the quasi SV-wave depend on the direction of the permeability of the formation.

In the final numerical example, we analyze the effect of attenuation of the quasi-P waves on the variation of the vertical and horizontal permeability, and the angles of propagation for a frequency of $10 \mathrm{kHz}$ and a squirt-flow length of $2 \mathrm{~mm}$. The attenuation curves in Figures $3 \mathrm{a}-\mathrm{c}$, were produced for angles of propagation of $30^{\circ}, 45^{\circ}$, and $60^{\circ}$. Each set of curves was produced by varying the horizontal permeability from $1 \mathrm{md}$ to $100 \mathrm{md}$ with steps of $1 \mathrm{md}$, and for vertical permeability $\tilde{\mathrm{k}}_{\mathrm{v}}=1,10$ and $20 \mathrm{md}$.

In this example, the curves show an increase of the attenuation to a maximum of $2 \mathrm{md}$ and a decrease of the attenuation as the horizontal permeability increases. In addition, the peaks of these attenuation curves are sensitive to the vertical permeability and the angle of propagation. In general, for the range of angles of propagation that we are considering, when the vertical permeability increases, the peaks of the attenuation curves become smaller. In the case of angles of propagation approaching the direction of the horizontal permeability, the atteruation is increased for values of $\tilde{\mathrm{k}}_{\mathrm{h}}$ greater than $10 \mathrm{md}$. For example, for this horizontal permeability value of $10 \mathrm{md}$ and $\tilde{\mathrm{k}}_{\mathrm{v}}=1 \mathrm{md}$, the attenuations are 0.026 and 0.028 for the angles of $30^{\circ}$ and $60^{\circ}$, respectively, and for values of $\tilde{\mathrm{k}}_{\mathrm{h}}$ near or equal to $2 \mathrm{md}$, the relation between the angle of propagation and attenuation is more difficult to predict. However, for practical situations in which the horizontal permeability is much greater than the vertical permeability, the attenuation will be maximum for angles of propagation perpendicular to the direction of vertical permeability. The example below describes a practical situation in which the seismic waves propagate parallel to the direction of maximum permeability between wells.

\section{Dispersion of Seismic Waves in a Fluvial Sandstone Formation in Oklahoma}

To demonstrate the applicability of the present poroelastic solution, interwell seismic waveforms recorded at the Gypsy test site, Oklahoma, were analyzed (Parra et al., 1994a). The geology and the petrophysics of the Gypsy sandstone formation have been reported by Doyle and Sweet (1995) and Parra, et al., (1994b). Most of the petrophysical analysis given in Doyle and Sweet (1995) was based on the Gypsy outcrop site which is located west of Tulsa. A brief description about the Gypsy sandstone reservoir properties that may affect the seismic wave propagation in the Gypsy sandstone is given below (Collier, 1994).

The Gypsy sandstone interval shown in Figure 4 is the strata between the regional unconformity on the Tallant marine sandstone and the flooding surface at the top of the Gypsy interval (about a depth of $910 \mathrm{ft}(280 \mathrm{~m})$ in well $7-7)$. The Gypsy sandstone zone is composed of stacked 
channel-fill point bar sandstones, mudstone, and siltstone bodies. The Gypsy sandstone exhibits

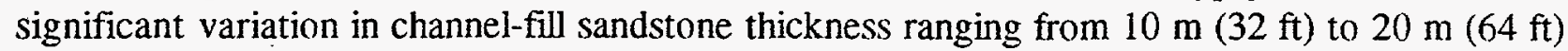
within a well separation of $150 \mathrm{~m}$ (Doyle and Sweet, 1995), and the mudstone and siltstone bodies constitute the architectural elements on a scale comparable to those defining the channel sandstone bodies. The well logs and core alone are not sufficient for demonstrating the degree of continuity of those zones defining the channel sandstone bodies, and thereby the extent to which they are barriers to vertical movement of fluids. However, pulse well-test data have demonstrated a good pressure communication between sand bodies and no communication across the mudstone zones. An average horizontal permeability of about $1640 \mathrm{md}$ was determined from a Gypsy pilot-site hydraulic conductivity study.

The results of the work of Doyle and Sweet (1995) have showed that the internal arrangement of porosity and permeability within channel sand bodies is controlled by lithofacies architectures, and differences in permeability among these lithofacies are related in part to depositional fabric. Specifically, cross-bedded and planar-laminated sandstones have the highest average permeability, whereas the average permeability of the mudclast and ripple-laminated sandstone is much lower. For example, in the Gypsy sandstone outcrop, the average permeability of the sandstone is three order of magnitude greater than the average permeability for the mudstone and siltstones, which are approximately $0.33 \mathrm{md}$. Since mudstones have very low permeabilities, they potentially exert a significant effect on the vertical permeability. The average vertical permeability $\left(\tilde{\mathrm{k}}_{\mathrm{v}}\right)$ is much smaller than the horizontal permeability $\left(\tilde{\mathrm{k}}_{\mathrm{h}}\right)$ in the Gypsy sandstone.

To relate the permeability to seismic wave propagation characteristics, interwell seismic signatures recorded for source-independent seismic attenuation and dispersion studies were analyzed (Parra, et al., 1994a). Two seismic traces were selected for this analysis; the first trace in Figure 5a was recorded in the receiver well $7-11$ at $75 \mathrm{~m}$ from the source well $7-1$, and the second trace in Figure $5 \mathrm{~b}$ was recorded in the receiver well 7-7 at $150 \mathrm{~m}$ from the source well $7-1$. The source and the two receiver wells are located in the same vertical plane. The receiver well 7-11 is between the source well 7-1 and the second receiver well 7-7. Both seismic receivers were placed at a depth of $296 \mathrm{~m}(970 \mathrm{ft})$ for a source placed at a depth of $300 \mathrm{~m}(984 \mathrm{ft})$. The spectra of the seismic signatures are given in Figure 6. Energy loss can be observed in the spectra and in the phase velocity dispersion curve which was calculated using the spectral ratio method given by Ganley and Kanasewich (1980).

An example of a dispersion curve represented by a solid line is shown in Figure 7. This curve is plotted together with synthetic phase velocity curves that were produced using the modeling solution developed in this work. The material property and saturant parameters of the Gypsy sandstone are given in Table 1. These parameters were obtained from well logs, interwell seismic data, and core information (Collier, 1994; Parra, et al., 1994a; Doyle, 1992; and Brown, 1995). In general, the Gypsy interval is thick compared to the wavelength used in the crosswell seismic experiments. At the Gypsy sandstone interval, the wavefronts seem to have an average value which is characteristic of a thick interval. For this sand, there is not a big difference between the sonic log data and the crosswell data. The Gypsy interval has an average velocity ( determined from cross well data) close to the average velocity obtained by integrating the sonic log through the sandstone interval. The velocity variations of these logs may be caused by the presence of heterogeneities or 
scatters having dimensions less than or equal to the wavelength used in the cross-well seismic survey.

In Figure 7, the theoretical curves were produced for vertical permeability values of $\tilde{\mathrm{k}}_{\mathrm{v}}=1 \mathrm{md}$, and $5 \mathrm{md}$, respectively, and $\mathrm{a} \tilde{\mathrm{k}}_{\mathrm{h}}=1640 \mathrm{md}$. The analysis of the petrophysic data suggests that the vertical permeability $(1 \mathrm{md})$ is caused by the presence of mudstone and siltstone bodies, which are barriers to vertical fluid movement, and the horizontal permeability (1640 md) is controlled by cross-bedded and planar-laminated sandstone. The dispersion response calculated for $5 \mathrm{md}$ is used as a reference curve to show how sensitive the dispersion is to vertical permeability. The theoretical predictions show the influence of permeability anisotropy on the dispersion of seismic waves. Since the seismic waves propagate parallel to the directions of maximum permeability between wells, the dispersion values derived from seismic signatures are consistent with the theoretical model. The differences between the dispersion data and the model data may be due to the presence of heterogeneities captured by the phase velocity data derived from the seismic signatures. Also, these differences may be caused by events (that are interfering with the direct P-wave event) such as reflection and transmission effects produced by the presence of boundaries. The present solution can be described as a first-order method in determining the phase velocity distribution associated with permeability anisotropy in the Gypsy sandstone formation.

\section{CONCLUSIONS}

The analysis of elastic wave propagation in transversely poroelastic isotropic media including the Biot and the squirt-flow mechanisms has provided a new partial differential equation that relates the fluid pressure to the dilatation of the rock matrix and the elements of the squirt-flow tensor. The solution of the new coupled poroelastic wave equation was used to demonstrate how the attenuation and dispersion of the quasi-P and quasi-SV waves can be related to the permeability anisotropy. Model results show the sensitivity of the directional permeability on the attenuation and dispersion, in particular the decrease in attenuation when the wave travels perpendicular to the direction of maximum permeability. A field example of wave propagation in the fluvial Gypsy sandstone reservoir shows the behavior of the vertical permeability on the dispersion curves which explains the fluid flow characteristics between the wells at the site. The analytical solution predicted the dispersion of the seismic waves traveling in the sandstone reservoir and predicted how dispersion relates to permeability anisotropy. The solution can be easily extended to determine the pressure and the vector wave field by simulating synthetic seismograms and to develop the boundary value problem of a plane wave propagating in a multilayer medium. These extensions are presently under development and will be reported in the near future. 
Table 1. Formation and Saturant Parameters

Example

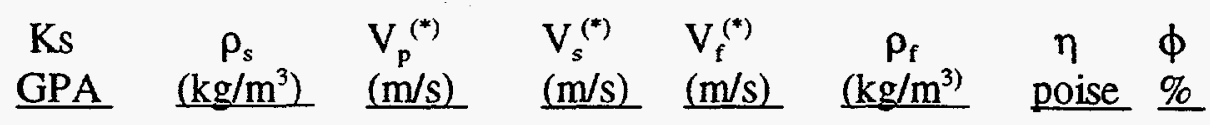

Dvorkin and

$\begin{array}{lllllllll}\text { Nur Model (1993) } & 38.0 & 2650 & 3969 & 2547 & 1500 & 1000 & 0.01 & 15\end{array}$

$\begin{array}{lllllllll}\text { Gypsy Model } & 37.9 & 2750 & 3149 & 1683 & 1500 & 1000 & 0.01 & 23\end{array}$

*The parameters $\mathrm{Vp}, \mathrm{V}_{\mathrm{s}}$ and $\mathrm{V}_{\mathrm{f}}$ are the compressional and shear wave velocity of the rock matrix, and the compressional wave velocity of the fluid, respectively.

\section{CAPTIONS}

Figure 1. The effect of frequency. a) Phase velocity; b) attenuation $\left(\mathrm{Q}^{-1}\right)$ for horizontal permeability, 1.25, 2.5, 3.75, and $5 \mathrm{md}$; and vertical permeability, $1.25 \mathrm{md}$. Squirt-flow length, $\mathrm{R}_{1}=\mathrm{R}_{3}=1 \mathrm{~mm}$.

Figure 2. The effect of angle of propagation. a) Quasi-P wave attenuation, and b) quasi-SV wave attenuation for frequencies between $500 \mathrm{~Hz}-4500 \mathrm{~Hz}$. The horizontal permeability $\tilde{\mathrm{k}}_{\mathrm{h}}=1000 \mathrm{md}$, and the vertical permeability $\tilde{\mathrm{k}}_{\mathrm{v}}=100 \mathrm{md}$. Squirt-flow length, $\mathrm{R}_{1}=\mathrm{R}_{3}=1 \mathrm{~mm}$..

Figure 3. The effect of the tensor permeability. Attenuation versus horizontal permeability for vertical permeability $\tilde{\mathrm{k}}_{\mathrm{v}}=1,10$ and $20 \mathrm{md}$ and angle of propagation of a) $30^{\circ}$; b) $45^{\circ}$; and c) $60^{\circ}$. Squirt-flow length, $R_{1}=R_{3}=2 \mathrm{~mm}$.

Figure 4. Well log display for well 7-7, Gypsy site, Oklahoma: core permeability (PERM), gamma ray (GR CORR), p-wave velocity (SONIC VE), density-neutron crossplot porosity correlated for shale volume (DNPHISHC) and core porosity (CORE PHI).

Figure 5. Common-source waveforms between wells (a) 7-1 and 7-11; (b) 7-1 and 7-7. Source at a depth of $300 \mathrm{~m}$ and detectors at $296 \mathrm{~m}$.

Figure 6. Spectra of the common source waveforms given in Figure 5.

Figure 7. Comparison between observed and calculated phase velocity curves. Observed dispersion curve determined from the spectra given in Figure 6. Squirt-flow length, $R_{1}=R_{3}=5 \mathrm{~mm}$.

\section{REFERENCES}

Auriault, J.L., Borne, L., and Chambon, R., 1985, Dynamics of porous saturated media, checking of the generalized law of Darcy: J. Acoust. Soc. Am. 77, 1641-1650. 
Biot, M.A., 1955, Theory of elasticity and consolidation for a porous anisotropic solid: J. Appl. Phys., 26, 182-185.

Biot, M.A., 1956, Theory of propagation of elastic waves in fluid-saturated porous rock: I. Low-frequency range: J. Acoust. Soc. Am., 28, 167-178.

Biot, M.A., 1962a, Mechanics of deformations and acoustic propagation in porous media: J. Appl. Phys., 33, 1482-1498.

Biot, M.A., 1962b, Generalized theory of acoustic propagation in porous dissipative media: J. Acoust. Soc. Am., 34, 1254-1264.

Brown, R.L., 1995, Integrating and coordinating GRI's continuity logging project at the Gypsy site: Oklahoma Geol. Sur. Final Report, Gas Research Institute, Contract No. 5093-260-2600.

Collier, H.G., 1994, Using guided waves to characterize gas reservoir continuity phase I: catalog of geological and petrophysical data from the Gypsy test site: Topical Report, Gas Research Institute, Report No. GRI-94/0384.4.

Doyle, J.D., and Sweet, M.L., 1995, Three-dimensional distribution of lithofacies, bounding surfaces, porosity and permeability in a fluvial sandstone, Gypsy sandstone of Northern Oklahoma: Am. Assn. Petr. Geol. Bulletin, 79, 70-96.

Doyle, J.D., 1992, Summary of petrophysical measurements made at the outcrop and subsurface sites: BP Exploration Report No. H0920074, 96.

Dvorkin, J., and Nur, A. 1993, Dynamic poroelasticity: A unified model with the squirt and the Biot mechanisms: Geophysics, 58, 523-533.

Ganley, D.C., and Kanasewich, E.R. 1989, Measurements of absorption and dispersion from check shot surveys: J. Geophys. Res., 85, 5219-5226.

Kazi-Aoual, M., 1988, Green's functions in an infinite transversely isotropic saturated poroelastic medium: J. Acoust. Soc. Am., 84, 1883-1889.

O'Connell, R.J., Budiansky, 1977, Viscoelastic properties of fluid-saturated cracked solids: J. Geophys. Res., 82, 5719-5736.

Parra, J.O., 1991, Analysis of elastic wave propagation in stratified fluid-filled porous media for interwell seismic applications: J. Acoust. Soc. Am., 90, 2557-2575.

Parra, J.O. and Xu, P.-C., 1994, Dispersion and attenuation of acoustic guided waves in layered fluid-filled porous media: J. Acoust. Soc. Am., 95, 91-98.

Parra, J.O., Zook, B.J., and Sturdivant, V.R., 1994a, Analysis and evaluation of interwell seismic logging techniques for hydrocarbon reservoir characterization: Final Report, Bartlesville Project Office, U.S. Department of Energy, Bartlesville, Oklahoma, Contract No. DE-AC22-90BC14649.

Parra, J.O., Zook, B.J., and Collier, H., 1994b, Seismic-guided waves for reservoir continuity at the Gypsy test site, Oklahoma: Presented at the 64th Ann. Internat. Mtg., Soc. Expl. Geophys. Expanded Abstracts, 755-759.

Schmitt, D.P., 1988, Effects of radial layering when logging in saturated porous formations: J. Acoust. Soc. Am., 84, 2200-2214.

Schmitt, D.P., 1989, Acoustic multipole logging in transversely isotropic poroelastic formations: J. Acoust. Soc. Am., 86, 2397-2421.

Tang, X.M., and Chelini, V., 1993, Fast inversion of formation permeability from Stoneley wave logs using simplified Biot-Rosenbaum model: Presented at the 63rd Ann. Internat. Mtg., Soc. Expl. Geophys., Expanded Abstracts, 80-83. 


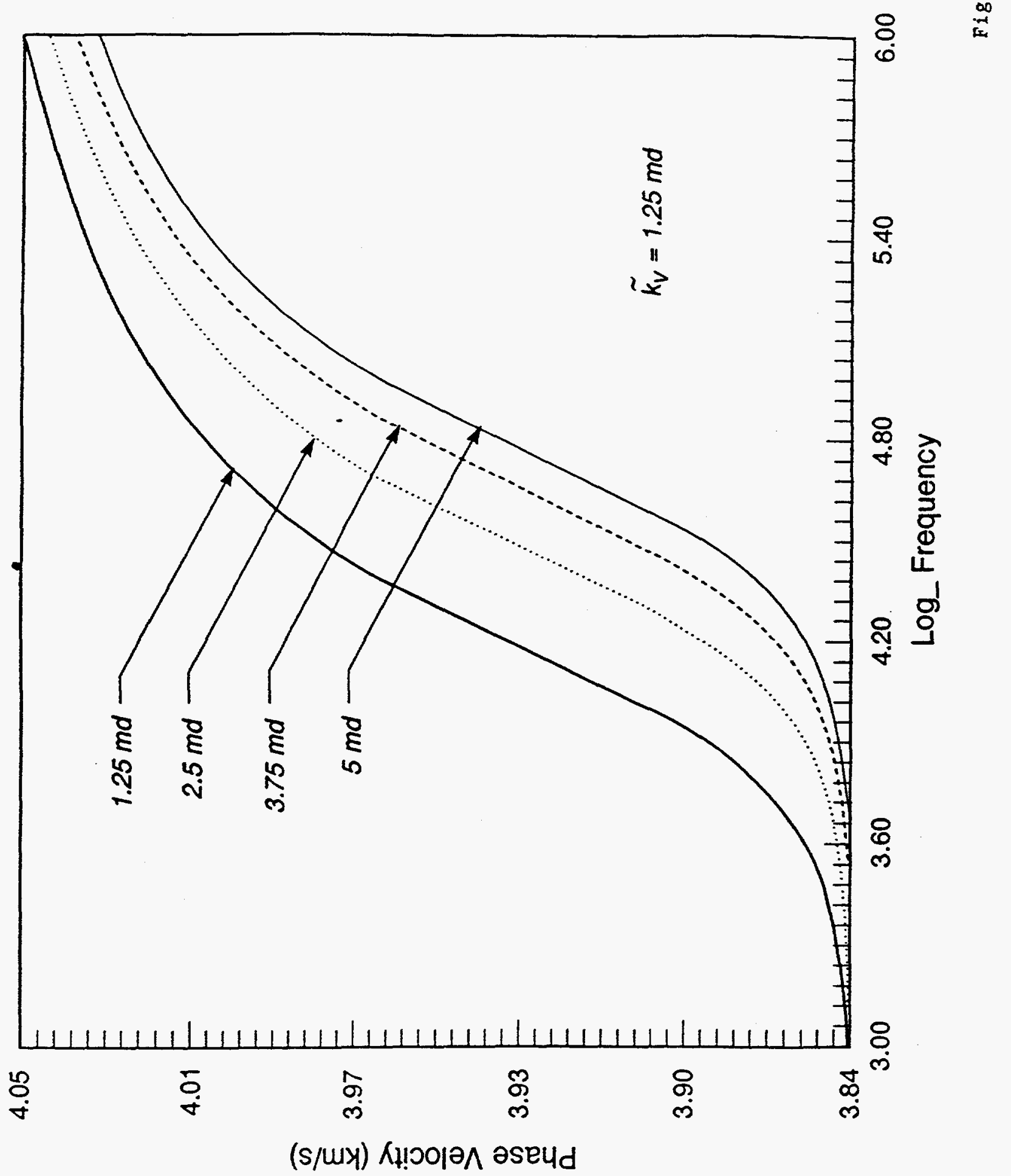




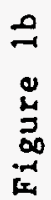

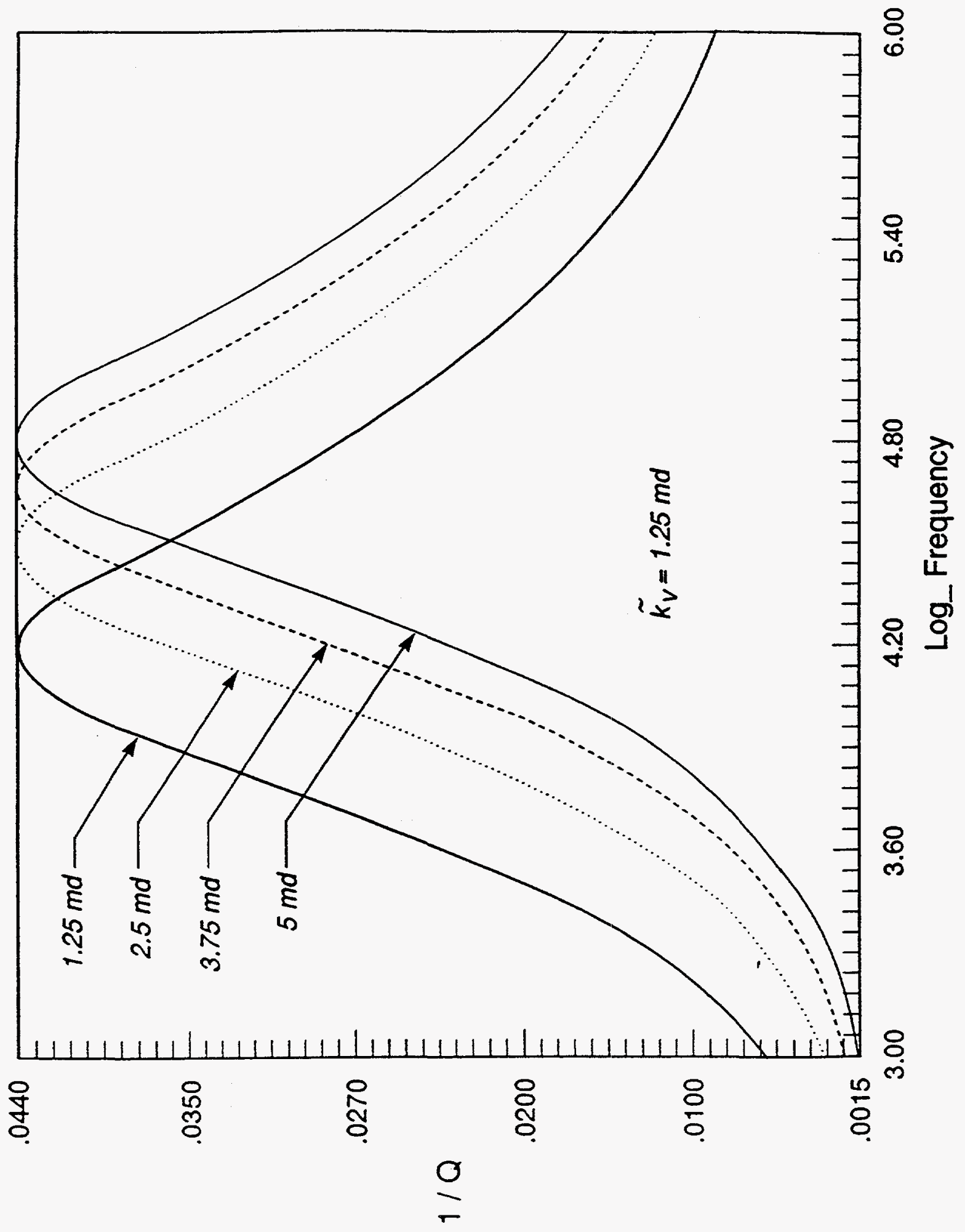




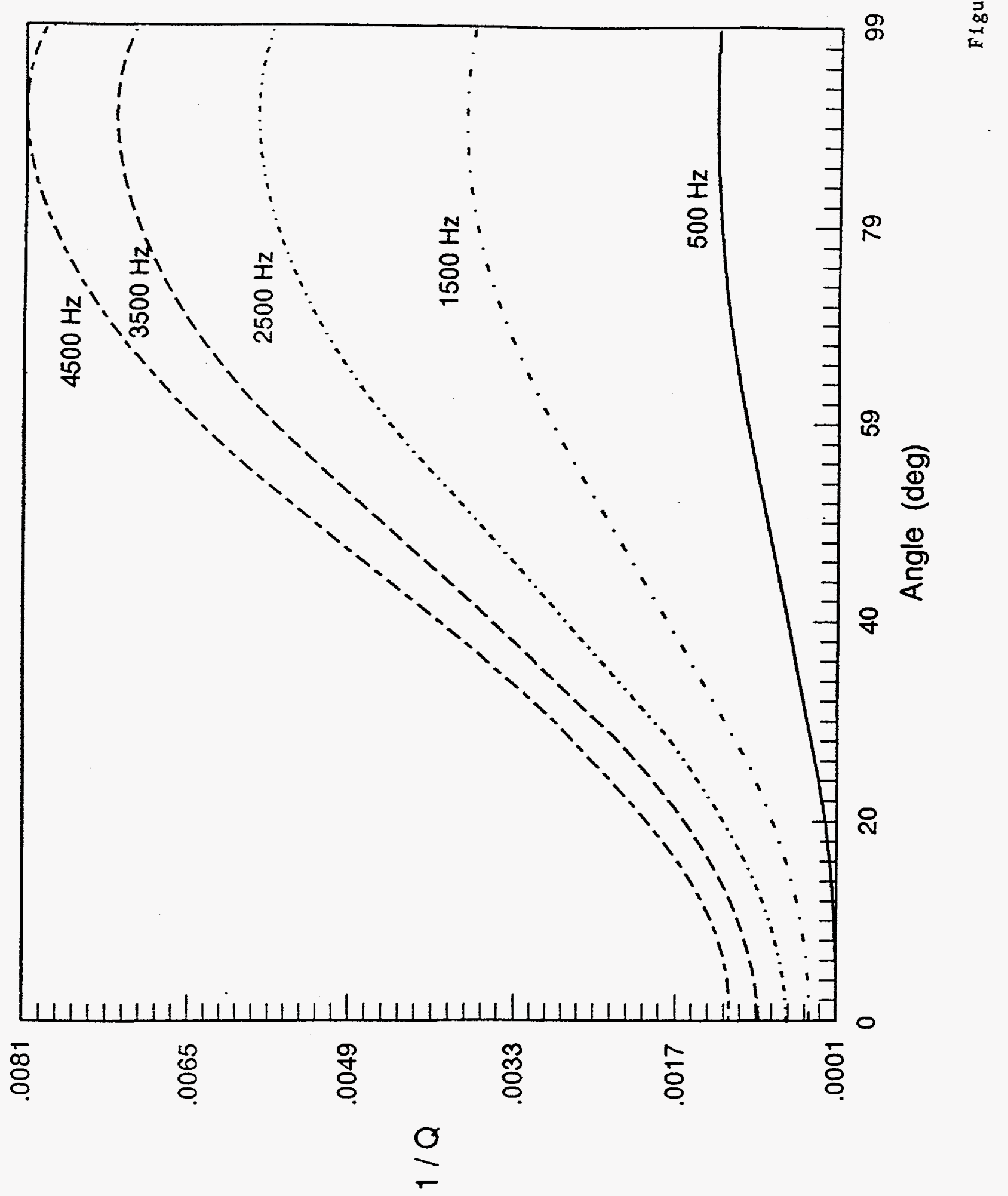




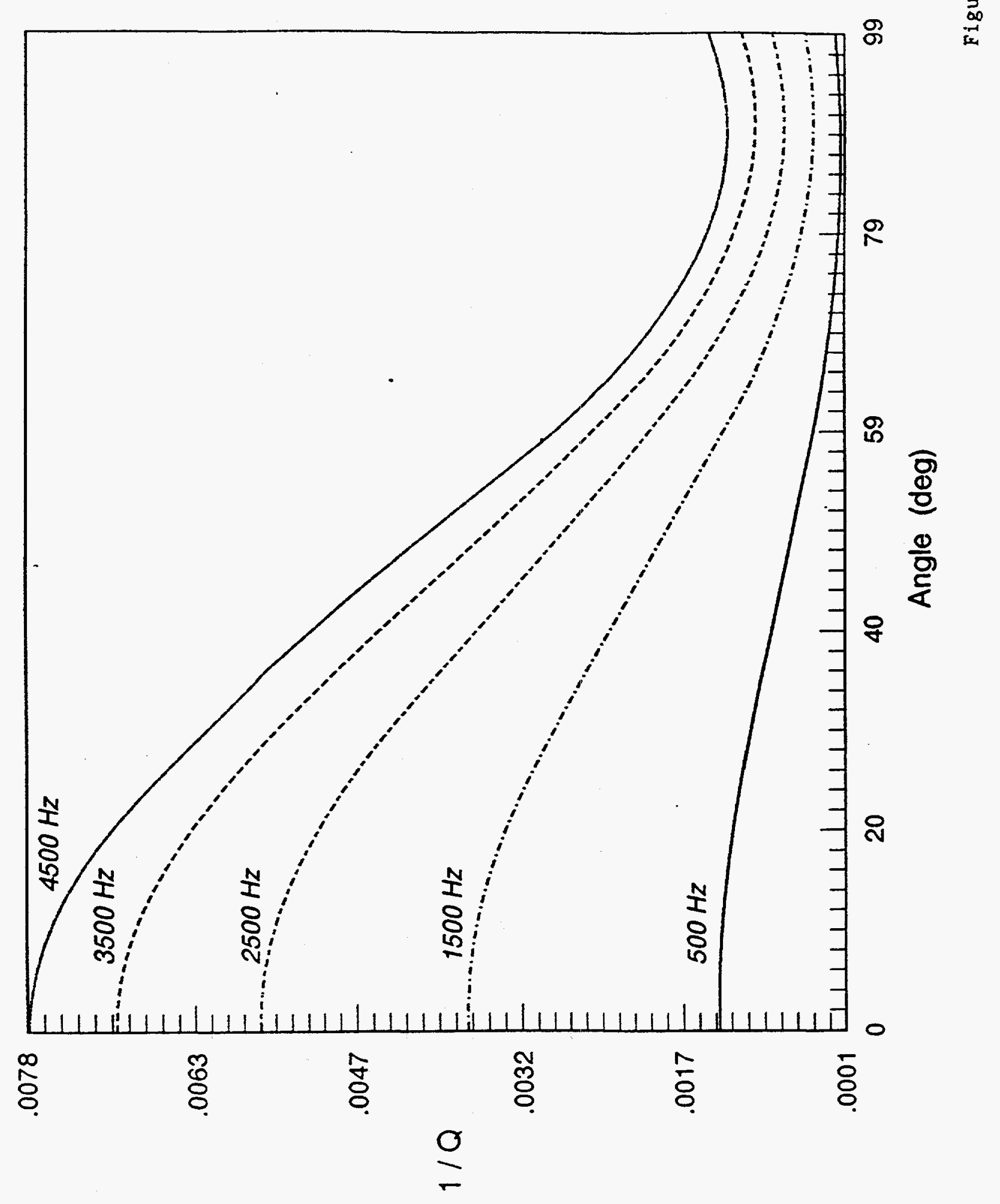




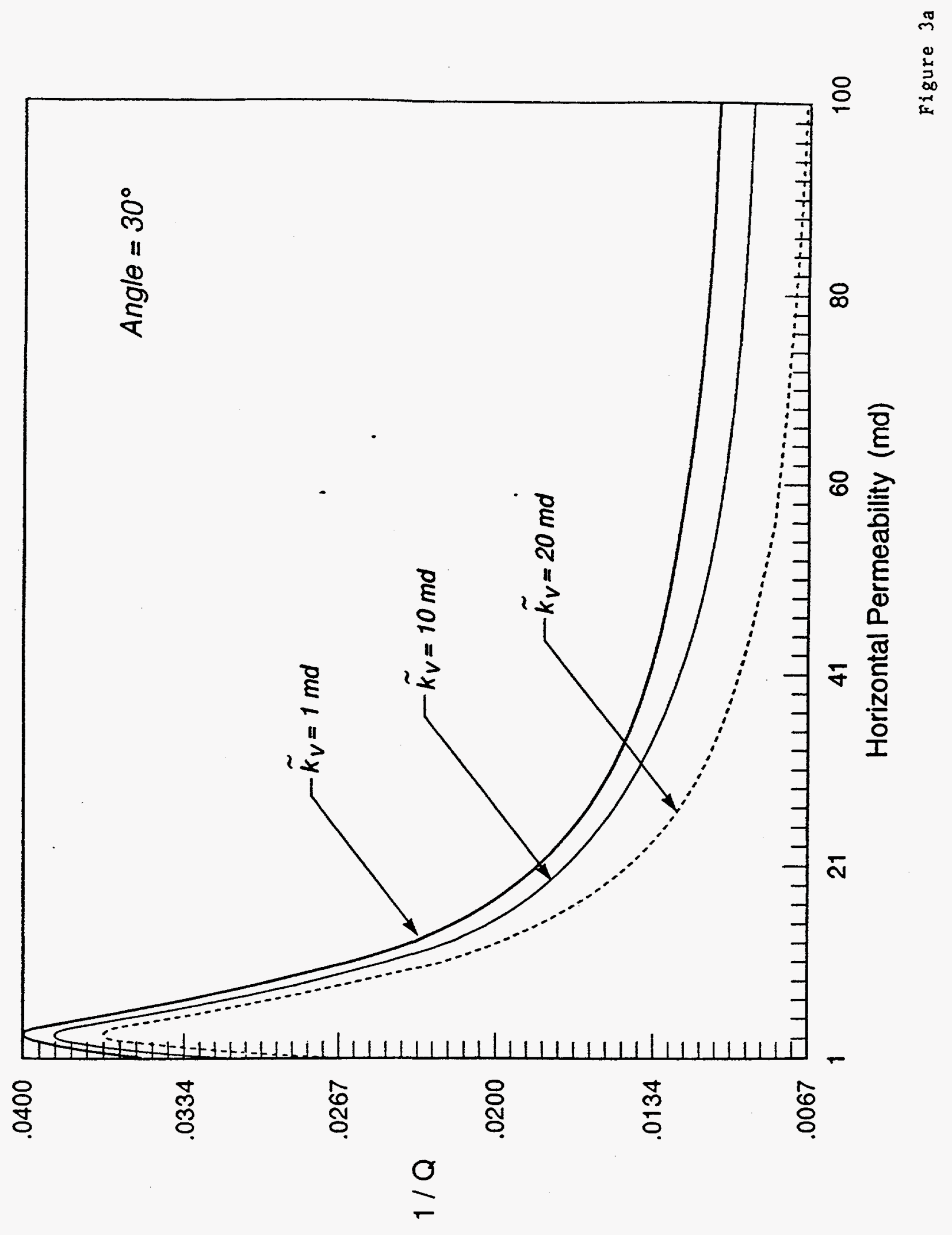




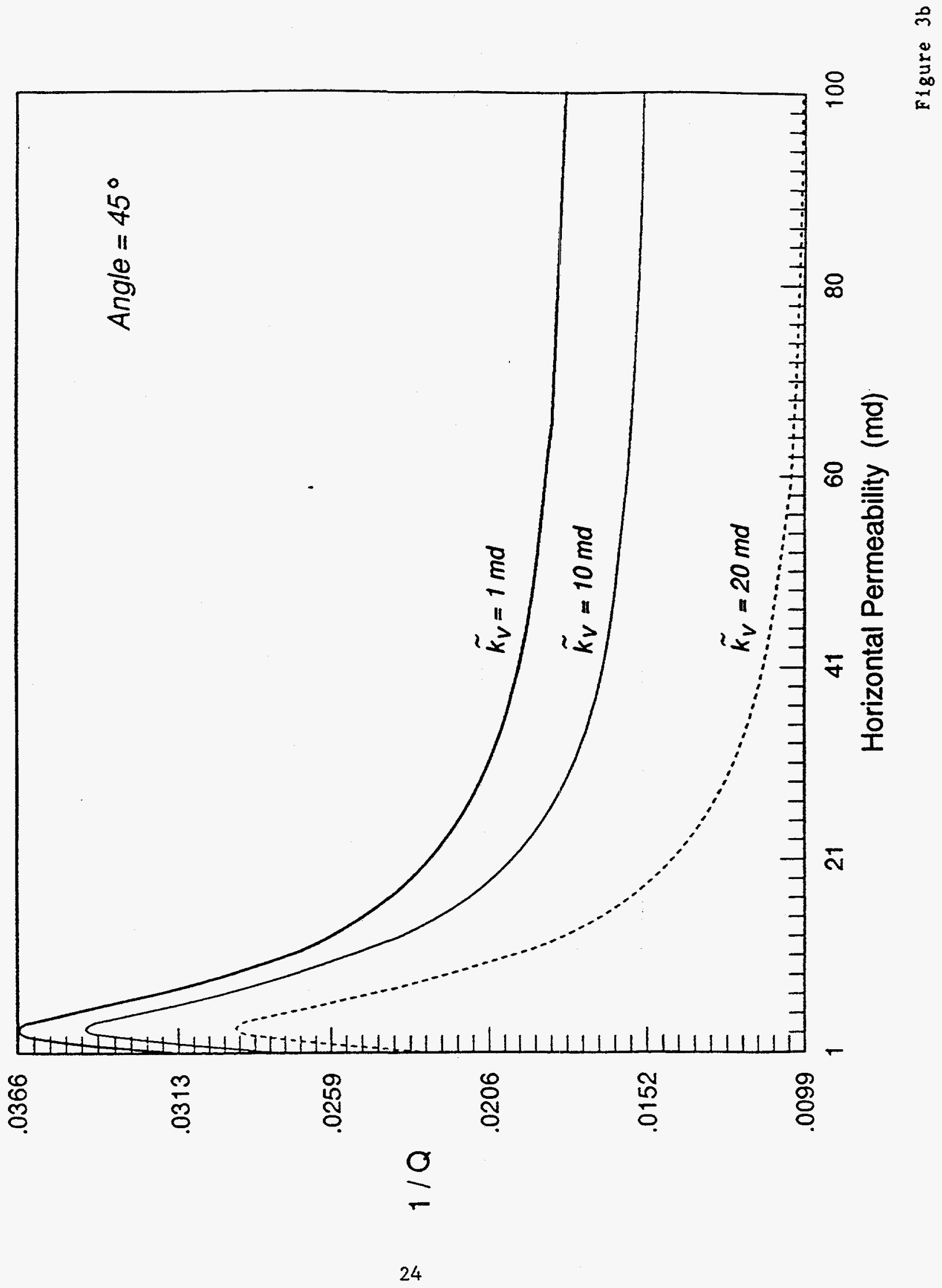




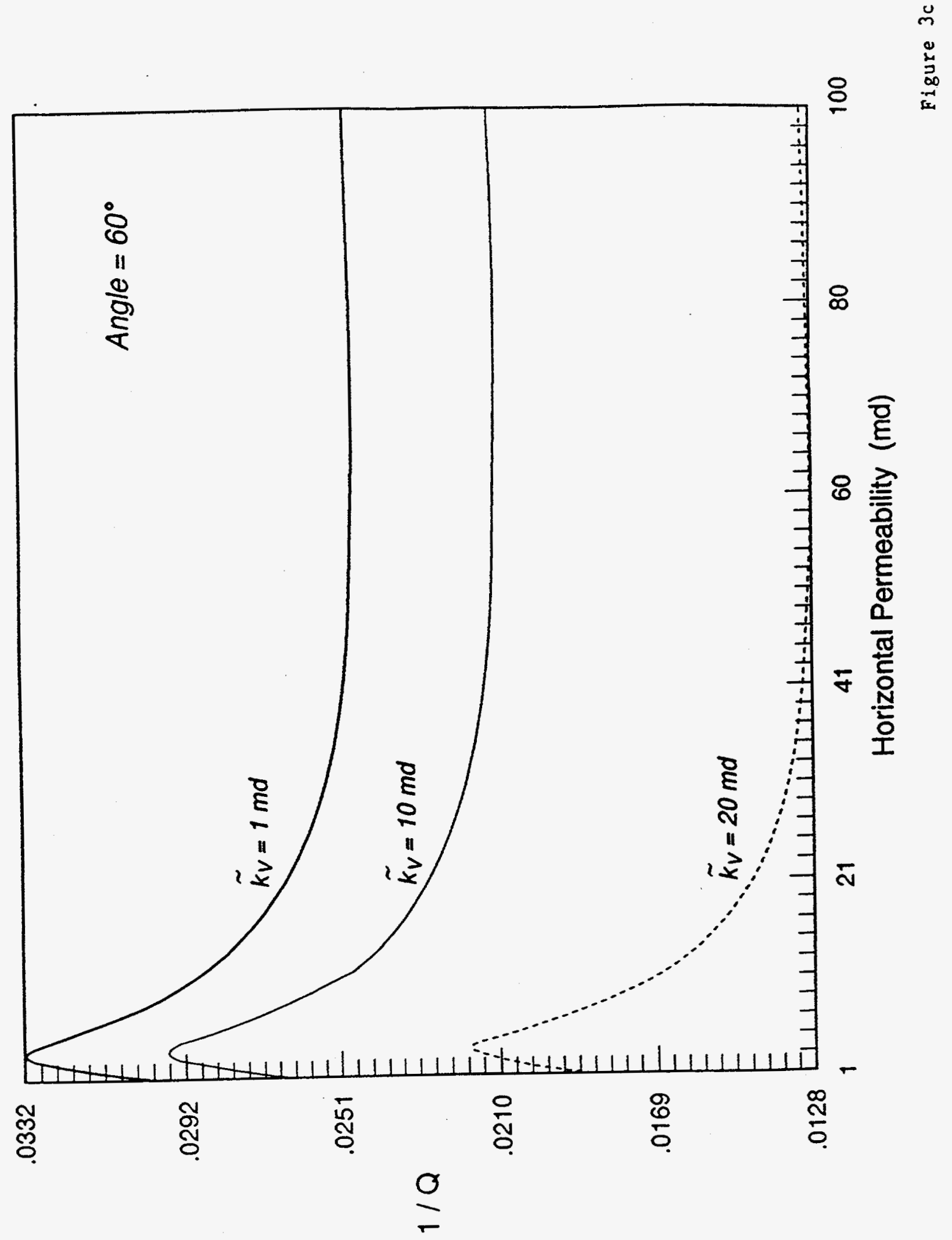


Well Name: DALLAS $7-7$

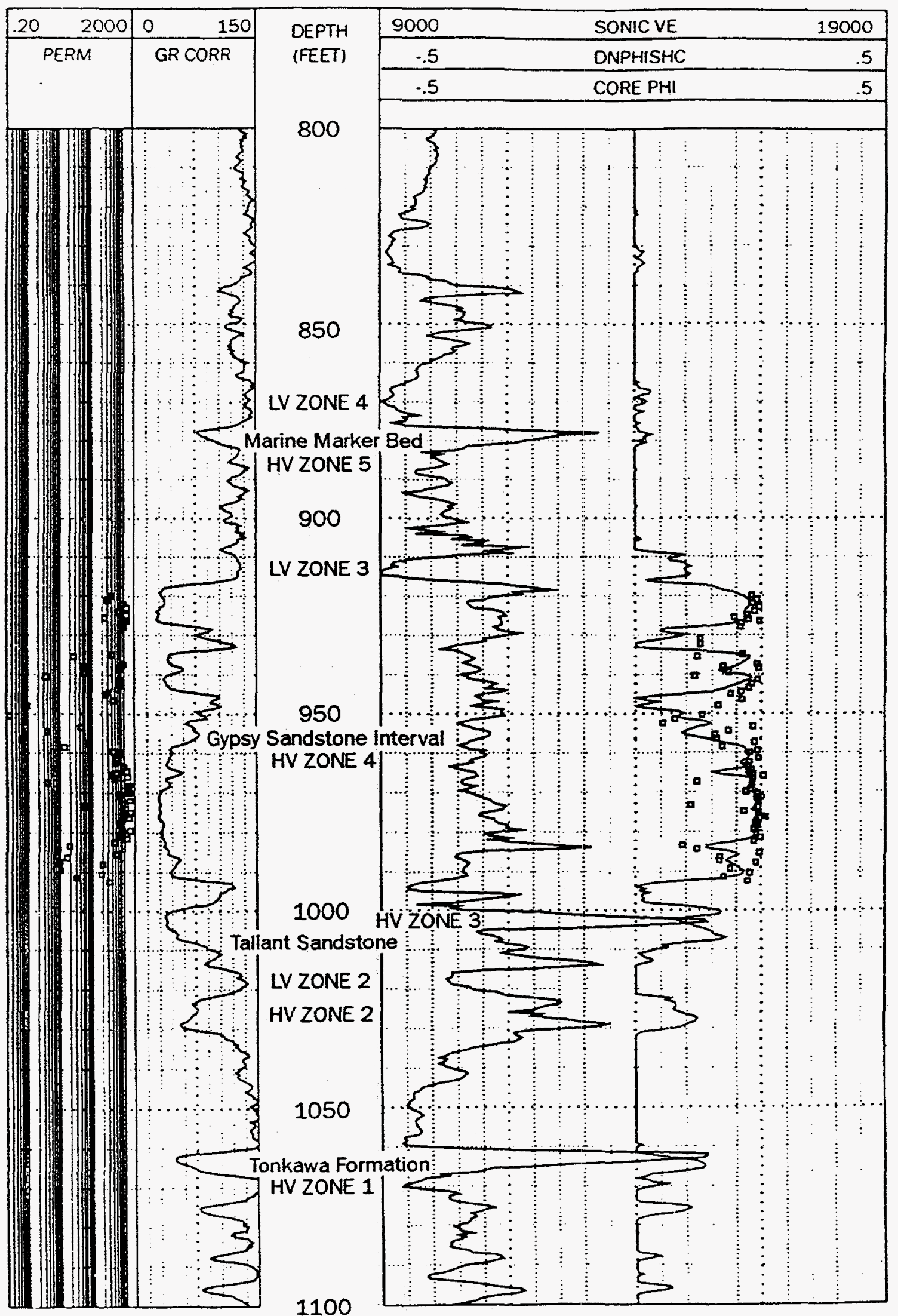




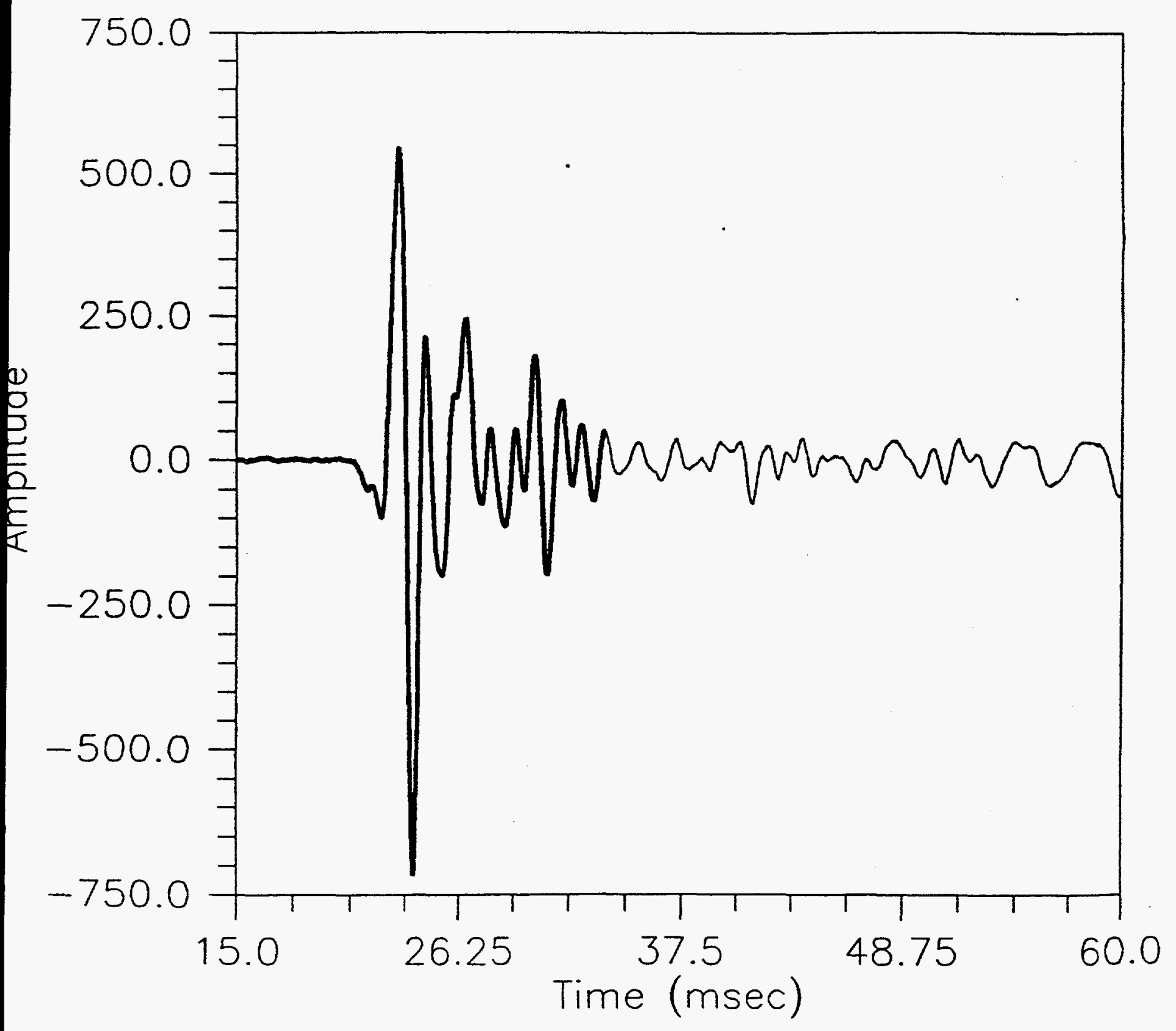

Figure Sa. 


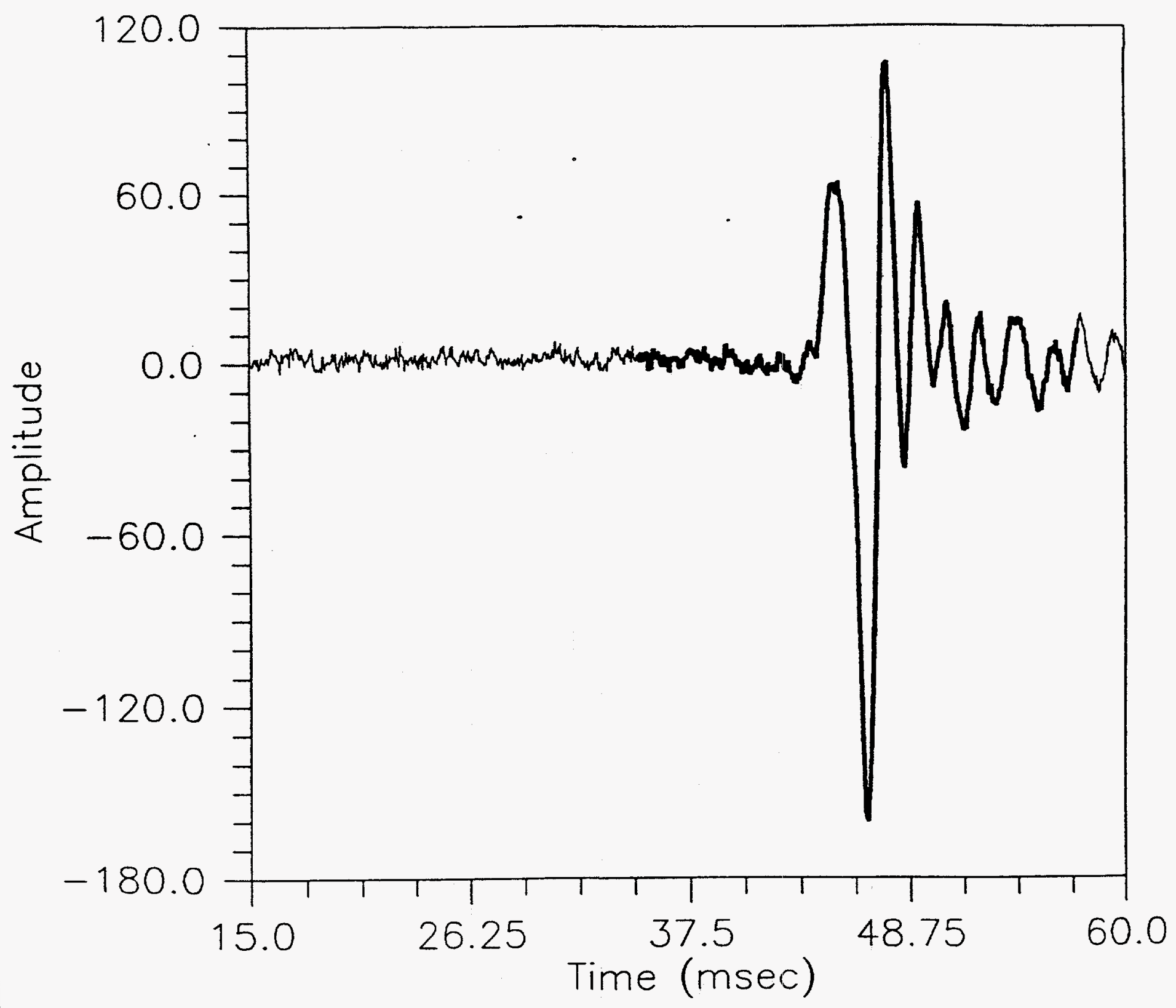




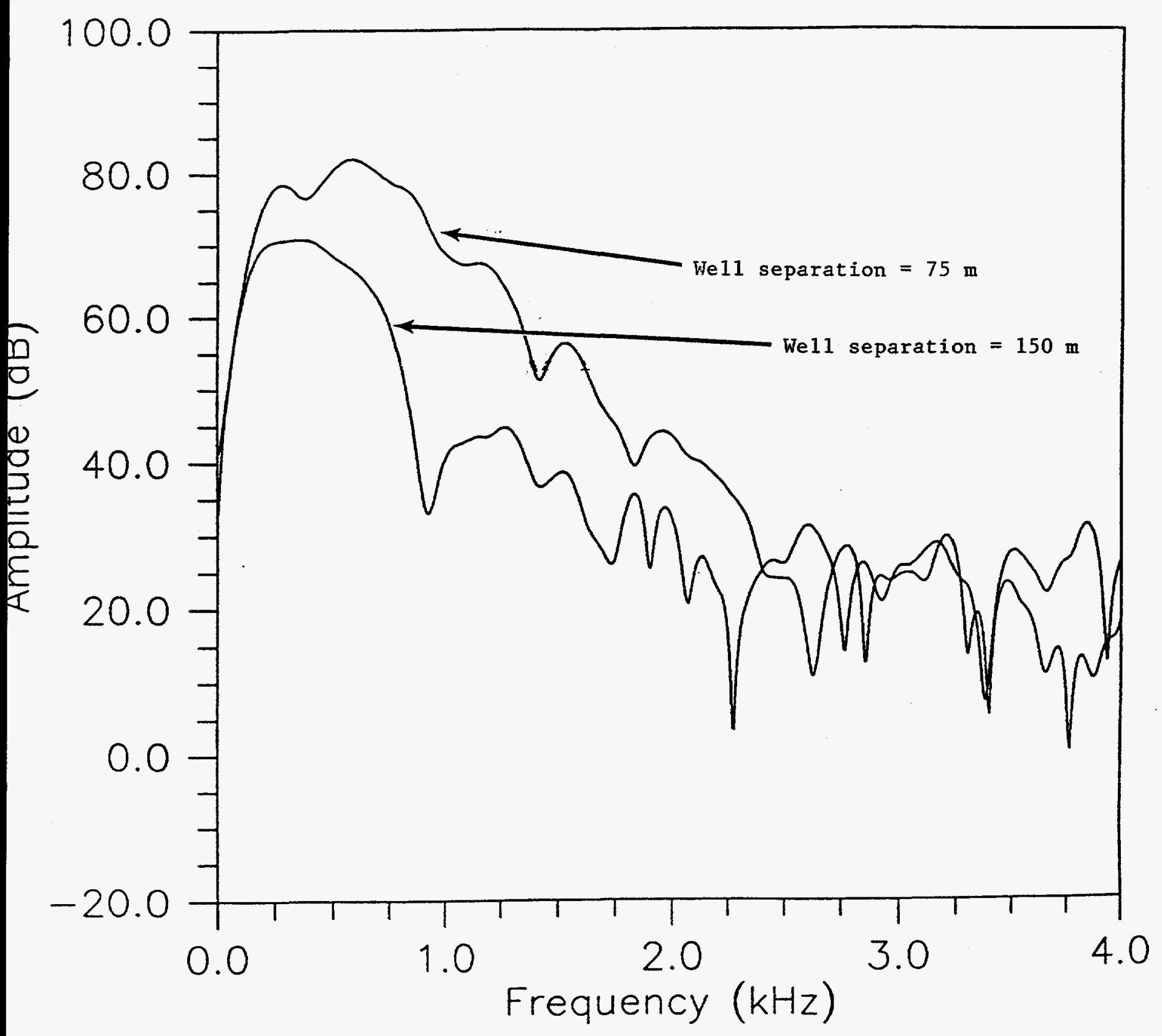

Figure 6 


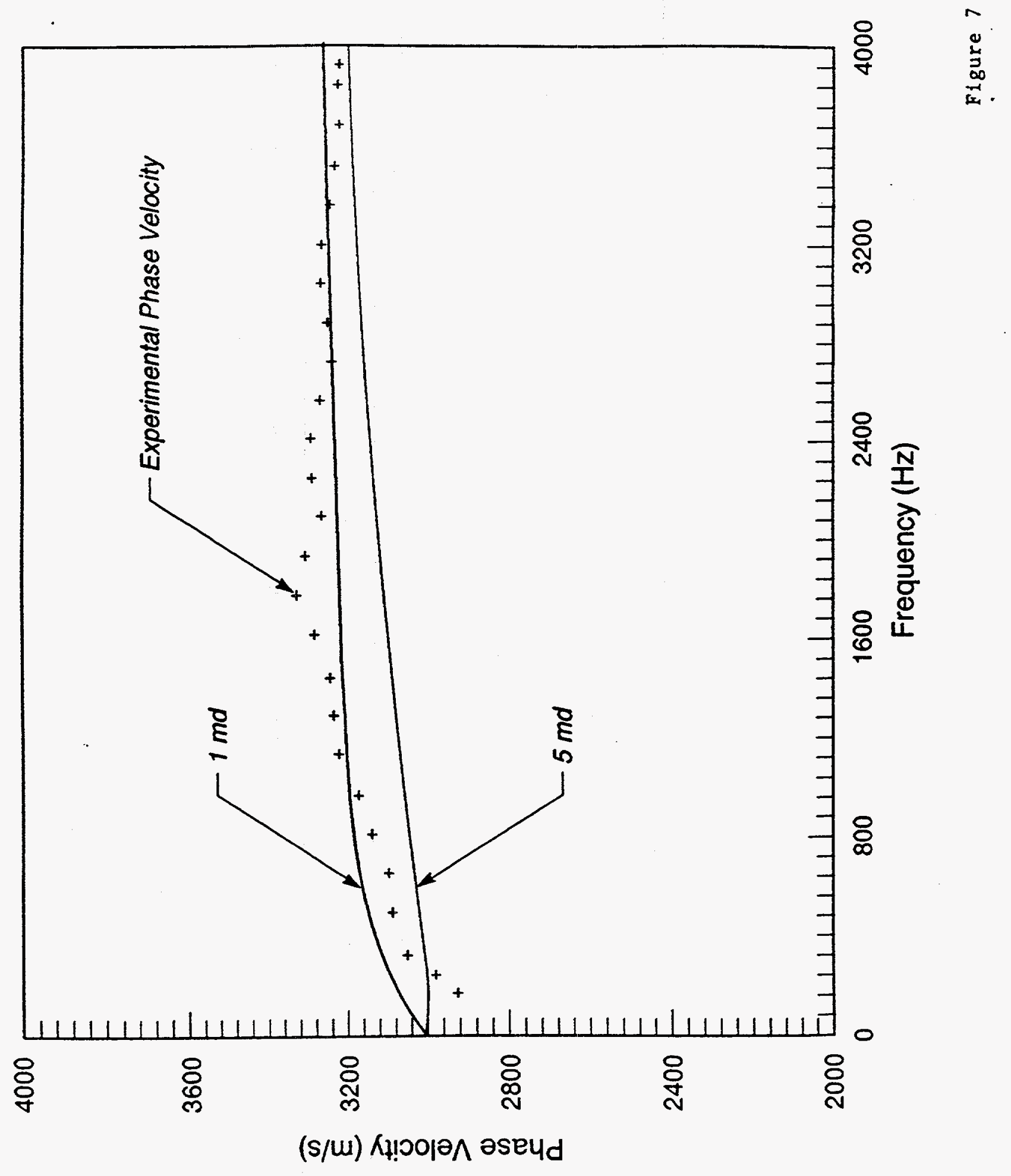




\section{B. Analysis of a Layered Transversely Isotropic Poroelastic Medium (LTIPM) to Incident Plane Waves}

The theory of propagation of elastic waves in fluid-filled saturated anisotropic porous media has been modified in accordance with the homogenized theory by Kazi-Azoual ${ }^{[3]}$, et al. 1988 , which introduces a second rank complex tensor permeability. The constitutive relations and balance equations of the modified Biot theory in the frequency-domain are:

\section{Classical Biot Equations}

\section{Constitutive Stress-Displacement Laws}

(i) Total stress in the porous medium

$$
\tau=\mathrm{Ce}-\alpha p
$$

(ii) Stress in the pore fluid

$$
\tau_{\mathrm{ij}}=-\phi p \delta_{\mathrm{ij}}=\frac{\phi}{\beta}[\alpha \mathbf{e}+\phi \nabla \cdot(\mathbf{U}-\mathbf{u})] \delta_{\mathrm{ij}}
$$

Momentum Balance Equation for total stress

$$
\frac{\partial \tau_{\mathrm{ij}}}{\partial \mathrm{x}_{\mathrm{j}}}=\nabla \cdot \tau=-\omega^{2}\left[\rho_{s}(1-\phi) \mathbf{u}+\phi \rho_{\mathrm{f}} \mathbf{U}\right]
$$

\section{Generalized Darcy Law}

$$
\mathbf{w}=\phi(\mathbf{U}-\mathbf{u})=-\frac{\mathbf{K}(\omega)}{i \omega}\left[\omega^{2} \rho_{\mathrm{f}} \mathbf{u}-\nabla \mathrm{p}\right]
$$

where

$$
\begin{array}{ll}
\mathbf{e} & =\text { strain tensor of porous medium } \\
\tau & =\text { stress tensor of porous medium } \\
\mathbf{C} & =\text { solid frame stiffness tensor } \\
\boldsymbol{\alpha} & =\text { second-rank elastic tensor } \\
\mathbf{p} & =\text { fluid pressure } \\
\beta & =\text { compressibility coefficient } \\
\mathbf{u} & =\text { displacement vector in the solid frame } \\
\mathbf{U} & =\text { displacement vector in the pore fluid } \\
\phi & =\text { porosity } \\
\rho_{\mathrm{s}} & =\text { solid density }
\end{array}
$$


$\rho_{\mathrm{f}} \quad=$ fluid density

$\mathbf{K}(\omega)=$ second-rank complex tensor of permeability

\section{Basic Equations for TIPM with $z$ Being Axis of Symmetry}

In this case, $C$ is given by

$$
\left[\begin{array}{cccccc}
C_{11} & C_{12} & C_{13} & 0 & 0 & 0 \\
C_{12} & C_{11} & C_{13} & 0 & 0 & 0 \\
C_{13} & C_{13} & C_{33} & 0 & 0 & 0 \\
0 & 0 & 0 & C_{44} & 0 & 0 \\
0 & 0 & 0 & 0 & C_{44} & 0 \\
0 & 0 & 0 & 0 & 0 & C_{66}
\end{array}\right]
$$

where we have defined

$$
\mathrm{C}_{66}=\frac{1}{2}\left(\mathrm{C}_{11}-\mathrm{C}_{12}\right)
$$

In the rest of the text, we use alternative notations $\{x, y, z\}$ for $\left\{x_{1}, x_{2}, x_{3}\right\}$, and $\left\{u_{x}, u_{y}, u_{z}\right\}$ for $\left\{u_{1}, u_{2}, u_{3}\right\}$.

The coupled system of equations for $u_{\mathrm{x}}, u_{\mathrm{z}}$ and $p$ is

$$
\begin{array}{r}
{\left[\mathrm{C}_{11} \frac{\partial^{2}}{\partial \mathrm{x}^{2}}+\mathrm{C}_{44} \frac{\partial^{2}}{\partial \mathrm{z}^{2}}+\omega^{2} \overline{\rho_{1}}\right] \mathrm{u}_{\mathrm{x}}+\left(\mathrm{C}_{13}+\mathrm{C}_{44}\right) \frac{\partial^{2}}{\partial \mathrm{x} \partial \mathrm{z}} \mathrm{u}_{\mathrm{z}}-\overline{\alpha_{1}} \frac{\partial \mathrm{p}}{\partial \mathrm{x}}=0} \\
\left(\mathrm{C}_{13}+\mathrm{C}_{44}\right) \frac{\partial^{2} \mathrm{u}_{\mathrm{x}}}{\partial \mathrm{x} \partial \mathrm{z}}+\left[\mathrm{C}_{44} \frac{\partial^{2}}{\partial \mathrm{x}^{2}}+\mathrm{C}_{33} \frac{\partial^{2}}{\partial \mathrm{z}^{2}}+\omega^{2} \bar{\rho}_{3}\right] \mathrm{u}_{\mathrm{z}}-\overline{\alpha_{3}} \frac{\partial \mathrm{p}}{\partial \mathrm{z}}=0 \\
-\bar{\alpha}_{1} \frac{\partial \mathrm{u}_{\mathrm{x}}}{\partial \mathrm{x}}-\overline{\alpha_{3}} \frac{\partial \mathrm{u}_{\mathrm{z}}}{\partial \mathrm{z}}+\left[\theta_{1} \frac{\partial^{2}}{\partial \mathrm{x}^{2}}+\theta_{3} \frac{\partial^{2}}{\partial \mathrm{z}^{2}}-\beta\right] \mathrm{p}=0
\end{array}
$$

where 


$$
\begin{aligned}
\overline{\alpha_{1}} & =\alpha_{1}+\omega^{2} \rho_{\mathrm{f}} \theta_{1} \\
\overline{\rho_{1}} & =\rho+\omega^{2} \rho_{\mathrm{f}}^{2} \theta_{1} \\
\rho & =\rho_{s}(1-\phi)+\phi \rho_{\mathrm{f}} \\
\theta_{1} & =-\frac{K_{1}(\omega)}{i \omega} \\
1 & =1,3
\end{aligned}
$$

The uncoupled equation for $u_{\mathrm{y}}$ is

$$
\left[C_{66} \frac{\partial^{2}}{\partial x^{2}}+C_{44} \frac{\partial^{2}}{\partial z^{2}}+\omega^{2} \overline{\rho_{1}}\right] u_{y}=0
$$

\section{Plane Wave Solution for TIPM with $z$ Being Axis of Symmetry}

Assume that

$$
\left\{\begin{array}{llll}
U_{x} & U_{y} & U_{z} & P
\end{array}\right\}^{T}=\left\{\begin{array}{llll}
u_{x} & u_{y} & u_{z} & p
\end{array}\right\}^{T} \exp \{i \xi[x \sin \phi+z \cos \phi-c t]\}
$$

where $c=\omega / \xi$ is the phase velocity and $k=\xi \sin \phi$ is the wavenumber in $x$-direction. Factor $\exp (-i \omega t)$ will be omitted in the rest of the text.

The above expression satisfies the equation of motion in $\mathrm{x}_{1}-\mathrm{x}_{3}$ plane if

$$
c^{6}+c^{4} \omega^{2} f_{2}(\omega, \phi)+c^{2} \omega^{4} f_{1}(\omega, \phi)+\omega^{6} f_{0}(\omega, \phi)=0
$$

where $f_{0}(\omega, \phi), f_{1}(\omega, \phi)$ and $f_{2}(\omega, \phi)$ are given in Appendix I. The three roots of $c^{2}$ defined by the above equation are denoted by $\mathrm{c}_{\delta}^{2}, \mathrm{c}_{\epsilon}{ }^{2}$, and $\mathrm{c}_{\gamma}{ }^{2}$.

For wave motion in $x_{2}$ direction, the phase velocity is given by

$$
c=c_{v}=\sqrt{\frac{C_{66} \sin ^{2} \phi+C_{44} \cos ^{2} \phi}{\overline{\rho_{x}}}}
$$

where

$$
\overline{\rho_{1}}=\rho_{s}(1-\phi)+\rho_{f} \phi+\omega^{2} \rho_{f}^{2} \theta_{1}
$$

The propagation wavenumbers associated with the quasi-P waves (fast and slow compressional waves), quasi-SV wave, and quasi-SH wave are then obtained as 


$$
\begin{aligned}
& \delta=\sqrt{\mathrm{k}^{2}-\omega^{2} / \mathrm{c}_{\delta}^{2}} \\
& \epsilon=\sqrt{\mathrm{k}^{2}-\omega^{2} / \mathrm{c}_{\epsilon}^{2}} \\
& \gamma=\sqrt{\mathrm{k}^{2}-\omega^{2} / \mathrm{c}_{\gamma}^{2}} \\
& \nu=\sqrt{\mathrm{k}^{2}-\omega^{2} / \mathrm{c}_{v}^{2}}
\end{aligned}
$$

In the isotropic case, Eq. (11) is reduced to

$$
\lambda_{1}^{2} \lambda_{2}^{2} \mathrm{k}_{s}^{2} \mathrm{c}^{6}-\mathrm{c}^{4} \omega^{2}\left[\lambda_{1}^{2} \lambda_{2}^{2}+\left(\lambda_{1}^{2}+\lambda_{2}^{2}\right) \mathrm{k}_{s}^{2}\right]+c^{2} \omega^{4}\left(\lambda_{1}^{2}+\lambda_{2}^{2}+\mathrm{k}_{s}^{2}\right)-\omega^{6}=0
$$

which can be further reduced to

$$
\left(k_{s}^{2} c^{2}-\omega^{2}\right)\left(\lambda_{1}^{2} c^{2}-\omega^{2}\right)\left(\lambda_{2}^{2} c^{2}-\omega^{2}\right)=0
$$

where

$$
\begin{aligned}
\lambda_{1}^{2}+\lambda_{2}^{2} & =\mathrm{k}_{\mathrm{p}}^{2}-\frac{\overline{\alpha^{2}}}{\theta(\lambda+2 \mu)}-\frac{\beta}{\theta} \\
\lambda_{1}^{2} \lambda_{2}^{2} & =-\frac{\mathrm{k}_{\mathrm{p}}^{2} \beta}{\theta} \\
\mathrm{k}_{s}^{2} & =\frac{\omega^{2} \bar{\rho}}{\mu} \\
\mathrm{k}_{\mathrm{p}}^{2} & =\frac{\omega^{2} \bar{\rho}}{\lambda+2 \mu}
\end{aligned}
$$

and

$$
\begin{aligned}
\lambda & =\mathrm{C}_{13} \\
\mu & =\mathrm{C}_{44}=\mathrm{C}_{66} \\
\lambda+2 \mu & =\mathrm{C}_{11}=\mathrm{C}_{33} \\
\theta & =\theta_{1}=\theta_{3} \\
\bar{\alpha} & =\overline{\alpha_{1}}=\overline{\alpha_{3}} \\
\bar{\rho} & =\overline{\rho_{1}}=\overline{\rho_{3}}
\end{aligned}
$$

Equations (1-18) can be found in Ref. [1]. 


\section{Basic Equations for TIPM with $x$ Being Axis of Symmetry}

For wave motion in transversely isotropic, poroelastic media with $x$-axis being the axis of symmetry, the basic equations may be obtained similarly. In the following, label 3 in the elastic and poroelastic constants remains associated with the symmetry axis of transverse isotropy of the material. However, this axis of symmetry coincides with the $x$-axis of the coordinate system. In this case, $C$ is rewritten as

$$
\left[\begin{array}{cccccc}
C_{33} & C_{13} & C_{13} & 0 & 0 & 0 \\
C_{13} & C_{11} & C_{12} & 0 & 0 & 0 \\
C_{13} & C_{12} & C_{11} & 0 & 0 & 0 \\
0 & 0 & 0 & C_{66} & 0 & 0 \\
0 & 0 & 0 & 0 & C_{44} & 0 \\
0 & 0 & 0 & 0 & 0 & C_{44}
\end{array}\right]
$$

The coupled system of equations for $u_{x}, u_{z}$ and $p$ is now given by

$$
\begin{array}{r}
{\left[\mathrm{C}_{33} \frac{\partial^{2}}{\partial \mathrm{x}^{2}}+\mathrm{C}_{44} \frac{\partial^{2}}{\partial \mathrm{z}^{2}}+\omega^{2} \overline{\rho_{3}}\right] \mathrm{u}_{\mathrm{x}}+\left(\mathrm{C}_{13}+\mathrm{C}_{44}\right) \frac{\partial^{2}}{\partial \mathrm{x} \partial \mathrm{z}} \mathrm{u}_{\mathrm{z}}-\overline{\alpha_{3}} \frac{\partial \mathrm{p}}{\partial \mathrm{x}}=0} \\
\left(\mathrm{C}_{13}+\mathrm{C}_{14}\right) \frac{\partial^{2} \mathrm{u}_{\mathrm{x}}}{\partial \mathrm{x} \partial \mathrm{z}}+\left[\mathrm{C}_{44} \frac{\partial^{2}}{\partial \mathrm{x}^{2}}+\mathrm{C}_{11} \frac{\partial^{2}}{\partial \mathrm{z}^{2}}+\omega^{2} \overline{\rho_{1}}\right] \mathrm{u}_{\mathrm{z}}-\overline{\alpha_{1}} \frac{\partial \mathrm{p}}{\partial \mathrm{z}}=0 \\
-\bar{\alpha}_{3} \frac{\partial \mathrm{u}_{\mathrm{x}}}{\partial \mathrm{x}}-\overline{\alpha_{1}} \frac{\partial \mathrm{u}_{\mathrm{z}}}{\partial \mathrm{z}}+\left[\theta_{3} \frac{\partial^{2}}{\partial \mathrm{x}^{2}}+\theta_{1} \frac{\partial^{2}}{\partial \mathrm{z}^{2}}-\beta\right] \mathrm{p}=0
\end{array}
$$

which leads to

$$
\left[\begin{array}{ccc}
\mathrm{C}_{33} \mathrm{k}^{2}+\mathrm{C}_{44} \zeta^{2}-\omega^{2} \overline{\rho_{3}} & \left(\mathrm{C}_{13}+\mathrm{C}_{44}\right) \mathrm{k} \zeta & \mathrm{ik} \overline{\alpha_{3}} \\
\left(\mathrm{C}_{13}+\mathrm{C}_{44}\right) \mathrm{k} \zeta & \left(\mathrm{C}_{44} \mathrm{k}^{2}+\mathrm{C}_{11} \zeta^{2}-\omega^{2} \overline{\rho_{1}}\right) & \mathrm{i} \zeta \overline{\alpha_{1}} \\
\mathrm{ik} \overline{\alpha_{3}} & \mathrm{i} \zeta \overline{\alpha_{1}} & \theta_{3} \mathrm{k}^{2}+\theta_{1} \zeta^{2}+\beta
\end{array}\right]\left\{\begin{array}{l}
\mathrm{u}_{\mathrm{x}} \\
\mathrm{u}_{\mathrm{z}} \\
\mathrm{p}
\end{array}\right\}=\left\{\begin{array}{l}
0 \\
0 \\
0
\end{array}\right\}
$$


And the uncoupled equation for $u_{\mathrm{y}}$ is

$$
\left[C_{44} \frac{\partial^{2}}{\partial x^{2}}+C_{66} \frac{\partial^{2}}{\partial z^{2}}+\omega^{2} \overline{\rho_{3}}\right] u_{y}=0
$$

\section{Plane Wave Solution for TIPM with $x$ Being Axis of Symmetry}

Again, we assume a plane wave solution:

$$
\left\{U_{x} \quad U_{y} \quad U_{z} P\right\}^{T}=\left\{\begin{array}{llll}
u_{x} & u_{y} & u_{z} & p
\end{array}\right\}^{T} \exp \{i \xi[x \sin \phi+z \cos \phi-c t]\}
$$

similar to Eq. (11),

The above expression satisfies the equation of motion in the $x-z$ plane if,

$$
c^{6}+c^{4} \omega^{2} g_{2}(\omega, \phi)+c^{2} \omega^{4} g_{1}(\omega, \phi)+\omega^{6} g_{0}(\omega, \phi)=0
$$

where $g_{0}(\omega, \phi), g_{1}(\omega, \phi)$ and $g_{2}(\omega, \phi)$ are the same as $f_{0}(\omega, \phi), f_{1}(\omega, \phi)$ and $f_{2}(\omega, \phi)$ given in the Appendix $A$, except that $C_{11}$ is switched with $C_{33}$ in the elastic constants, and subscript 1 with 3 in the poroelastic constants, and vise versa. The three roots of $c^{2}$ defined by the above equation are again denoted by $\mathrm{c}_{\delta}{ }^{2}, \mathrm{c}_{\epsilon}{ }^{2}$, and $\mathrm{c}_{\gamma}{ }^{2}$.

Denote

$$
\begin{aligned}
& \delta=\left(\mathrm{k}^{2}-\frac{\omega^{2}}{\mathrm{c}_{\delta}{ }^{2}}\right)^{\frac{1}{2}} \\
& \epsilon=\left(\mathrm{k}^{2}-\frac{\omega^{2}}{\mathrm{c}_{\epsilon}{ }^{2}}\right)^{\frac{1}{2}} \\
& \gamma=\left(\mathrm{k}^{2}-\frac{\omega^{2}}{\mathrm{c}_{\gamma}{ }^{2}}\right)^{\frac{1}{2}}
\end{aligned}
$$

Then Eq. (23) can be rewritten such that these roots are expressed in terms of wavenumber $\mathrm{k}$ instead of $\phi$ :

$$
\mathrm{P}_{6}(\mathrm{k}) \zeta^{6}+\mathrm{P}_{4}(\mathrm{k}) \zeta^{4}+\mathrm{P}_{2}(\mathrm{k}) \zeta^{2}+\mathrm{P}_{0}(\mathrm{k})=0
$$


where $\zeta^{2}$ represents the three pairs of roots, $-\delta^{2},-\epsilon^{2}$, or $-\gamma^{2}$, and

$$
\begin{aligned}
& \mathrm{P}_{6}(\mathrm{k})=\theta_{1} \\
& P_{4}(k)=\frac{\overline{\alpha_{1}}}{C_{11}}+S^{2}+\theta_{1}\left(\sigma^{2}+\chi^{2}+q^{2}\right) \\
& \mathrm{P}_{2}(\mathrm{k})=\frac{\overline{\alpha_{1}^{2}}}{\mathrm{C}_{11}}\left(\mathrm{k}^{2} \eta^{2}-\frac{\omega^{2} \overline{\rho_{3}}}{\mathrm{C}_{44}}\right)+\theta_{1} \mathrm{p}^{2}+\mathrm{s}^{2}\left(\sigma^{2}+\chi^{2}+\mathrm{q}^{2}\right) \\
& \mathrm{P}_{0}(\mathrm{k})=\frac{\overline{\alpha_{3}}}{\mathrm{C}_{11}}\left(\mathrm{k}^{2}-\frac{\omega^{2} \overline{\rho_{1}}}{\mathrm{C}_{44}}\right)+\mathrm{s}^{2} \mathrm{p}^{2} \\
& \sigma^{2}=k^{2}-\frac{\omega^{2} \overline{\rho_{1}}}{C_{11}} \\
& \chi^{2}=\mathrm{k}^{2}-\frac{\omega^{2} \overline{\rho_{3}}}{\mathrm{C}_{44}} \\
& \mathrm{~s}^{2}=\theta_{3} \mathrm{k}^{2}+\beta \\
& \eta^{2}=\frac{\mathrm{C}_{33}}{\mathrm{C}_{44}}+\frac{\mathrm{C}_{11}}{\mathrm{C}_{44}}\left(\frac{\overline{\alpha_{3}}}{\overline{\alpha_{1}}}\right)^{2}-2 \frac{\overline{\alpha_{3}}}{\overline{\alpha_{1}}} \frac{\mathrm{C}_{13}+\mathrm{C}_{44}}{\mathrm{C}_{44}} \\
& \mathrm{q}^{2}=\mathrm{k}^{2}\left[\frac{\mathrm{C}_{33}\left(\mathrm{C}_{33}-2 \mathrm{C}_{44}\right)-\mathrm{C}_{13}\left(\mathrm{C}_{13}+2 \mathrm{C}_{44}\right)}{\mathrm{C}_{11} \mathrm{C}_{44}}\right. \\
& \mathrm{p}^{2}=\frac{\mathrm{C}_{33}}{\mathrm{C}_{11}}\left(\frac{\mathrm{k}^{2}-\omega^{2} \overline{\rho_{1}}}{\mathrm{C}_{44}}\right)\left(\mathrm{k}^{2}-\frac{\omega^{2} \overline{\rho_{3}}}{\mathrm{C}_{33}}\right)
\end{aligned}
$$

In the elastic case, all the poroelastic constants vanish and $\theta_{1}$ becomes a common factor of all four coefficients of the cubic equation. As a result, the cubic equation is reduced to a quadratic equation $\left(\mathrm{P}_{0}=0\right)$.

given by

For wave motion in the $y$ direction, the phase velocity and wavenumber are

$$
\begin{aligned}
& c_{v}=\sqrt{\frac{C_{44} \sin ^{2} \phi+C_{66} \cos ^{2} \phi}{\overline{\rho_{3}}}} \\
& v=\left(k^{2}-\frac{\omega^{2}}{c_{v}{ }^{2}}\right)^{\frac{1}{2}}
\end{aligned}
$$




\section{Propagation of Plane Waves within the $x-z$ Plane in LTIPM with $x$ Being the Axis of Symmetry and Interfaces Parallel to the $x-y$ Plane}

The plane wave assumption defined by Eq. (10) allows the following form of solution for the four primary response components in a layer of the LTIPM.

$$
\left\{\begin{array}{l}
u_{x} \\
u_{y} \\
u_{z} \\
p
\end{array}\right\}=\left[\begin{array}{cccccccc}
1 & 1 & 1 & 0 & 1 & 1 & 1 & 0 \\
0 & 0 & 0 & 1 & 0 & 0 & 0 & 1 \\
R_{1} & R_{2} & R_{3} & 0 & R_{5} & R_{6} & R_{7} & 0 \\
Q_{1} & Q_{2} & Q_{3} & 0 & Q_{5} & Q_{6} & Q_{7} & 0
\end{array}\right]\left\{\begin{array}{c}
A_{1} e^{-\delta z} \\
A_{2} e^{-\epsilon z} \\
A_{3} e^{-\gamma z} \\
A_{4} e^{-v z} \\
A_{5} e^{\delta z} \\
A_{6} e^{\epsilon z} \\
A_{7} e^{\gamma z} \\
A_{8} e^{v z}
\end{array}\right\} e^{i k x}
$$

In Eq. (29), $A_{j}$ is the unknown coefficient associated with the $j$-th wavenumber in the z- direction, $R_{j}=u_{z} / u_{x}$, and $Q_{j}=p / u_{x}$, where the subscripts, $j=1,2,3 \ldots 8$, are corresponding to the three pairs of roots for $\zeta^{2}$ and one pair of roots for $v^{2}$. In each pair, the negative sign represents down-going wave and the positive sign represents the up-going wave.

These two ratios, $R_{j}$ and $Q_{j}$, can be obtained from Eq. (21), namely,

$$
\mathrm{R}_{\mathrm{j}}=\frac{\Delta_{\mathrm{R}}}{\Delta} \quad \mathrm{Q}_{\mathrm{j}}=\frac{\Delta_{\mathrm{Q}}}{\Delta}
$$

where

$$
\begin{aligned}
\Delta & =a_{22} a_{33}-a_{23} a_{32} \\
\Delta_{R} & =a_{23} a_{31}-a_{21} a_{33} \\
\Delta_{Q} & =a_{21} a_{32}-a_{22} a_{31} \\
a_{22} & =C_{44} k^{2}+C_{11} \zeta^{2}-\omega^{2} \overline{\rho_{1}} \\
a_{23} & =i \alpha_{1} \zeta \\
a_{32} & =i \alpha_{1} \zeta \\
a_{33} & =\theta_{3} k^{2}+\theta_{1} \zeta^{2}+\beta \\
a_{21} & =\left(C_{13}+C_{44}\right) k \zeta \\
a_{31} & =i k \alpha_{3}
\end{aligned}
$$


The stress and volumetric flux components are defined by Eqs. (1) and (4) as functions of the above primary response components, $\left\{u_{x}, u_{y}, u_{z}, p\right\}$; hence they can be expressed in terms of the unknown coefficients $A_{j}, j=1,2, \ldots 8$. In obtaining these expressions, the following rules have been applied:

$$
\frac{\partial}{\partial x}=\mathrm{ik} \quad \frac{\partial}{\partial z}=\mathrm{i} \zeta=\mp \delta, \mp \epsilon, \mp \gamma, \mp \nu
$$

These expressions are given below.

$$
\left\{\begin{array}{c}
\tau_{x x} \\
\tau_{y y} \\
\tau_{z z} \\
\tau_{y z} \\
\tau_{x z} \\
\tau_{x y} \\
w_{x} \\
w_{y} \\
w_{z}
\end{array}\right\}=\left[\begin{array}{cccccccc}
P_{1} & P_{2} & P_{3} & 0 & P_{5} & P_{6} & P_{7} & 0 \\
0 & 0 & 0 & S_{4} & 0 & 0 & 0 & S_{8} \\
T_{1} & T_{2} & T_{3} & 0 & T_{5} & T_{6} & T_{7} & 0 \\
0 & 0 & 0 & U_{4} & 0 & 0 & 0 & U_{8} \\
V_{1} & V_{2} & V_{3} & 0 & V_{5} & V_{6} & V_{7} & 0 \\
0 & 0 & 0 & W_{4} & 0 & 0 & 0 & W_{8} \\
X_{1} & X_{2} & X_{3} & 0 & X_{5} & X_{6} & X_{7} & 0 \\
0 & 0 & 0 & Y_{4} & 0 & 0 & 0 & Y_{8} \\
Z_{1} & Z_{2} & Z_{3} & 0 & Z_{5} & Z_{6} & Z_{7} & 0
\end{array}\right]\left\{\begin{array}{l}
A_{1} e^{-\delta z} \\
A_{2} e^{-\epsilon z} \\
A_{3} e^{-\gamma z} \\
A_{4} e^{-v z} \\
A_{5} e^{\delta z} \\
A_{6} e^{\epsilon z} \\
A_{7} e^{\gamma z} \\
A_{8} e^{v z}
\end{array}\right\} e^{i k x}
$$

where 


$$
\begin{aligned}
& \mathrm{P}_{1}=\mathrm{C}_{33} \mathrm{ik}-\mathrm{C}_{13} \delta \mathrm{R}_{1}-\overline{\alpha_{3}} \mathrm{Q}_{1} \quad \mathrm{P}_{2}=\mathrm{C}_{33} \mathrm{ik}-\mathrm{C}_{13} \in \mathrm{R}_{2}-\overline{\alpha_{3}} \mathrm{Q}_{2} \quad \mathrm{P}_{3}=\mathrm{C}_{33} \mathrm{ik}-\mathrm{C}_{13} \gamma \mathrm{R}_{3}-\overline{\alpha_{3}} \mathrm{Q}_{3} \\
& \mathrm{P}_{5}=\mathrm{C}_{33} \mathrm{ik}+\mathrm{C}_{13} \delta \mathrm{R}_{5}-\overline{\alpha_{3}} \mathrm{Q}_{5} \quad \mathrm{P}_{6}=\mathrm{C}_{33} \mathrm{ik}+\mathrm{C}_{13} \in \mathrm{R}_{6}-\overline{\alpha_{3}} \mathrm{Q}_{6} \quad \mathrm{P}_{7}=\mathrm{C}_{33} \mathrm{ik}+\mathrm{C}_{13} \gamma \mathrm{R}_{7}-\overline{\alpha_{3}} \mathrm{Q}_{7} \\
& \mathrm{~T}_{1}=\mathrm{C}_{13} \mathrm{ik}-\mathrm{C}_{11} \delta \mathrm{R}_{1}-\overline{\alpha_{1}} \mathrm{Q}_{1} \quad \mathrm{~T}_{2}=\mathrm{C}_{13} \mathrm{ik}-\mathrm{C}_{11} \in \mathrm{R}_{2}-\overline{\alpha_{1}} \mathrm{Q}_{2} \quad \mathrm{~T}_{3}=\mathrm{C}_{13} \mathrm{ik}-\mathrm{C}_{11} \gamma \mathrm{R}_{3}-\overline{\alpha_{1}} \mathrm{Q}_{3} \\
& \mathrm{~T}_{4}=\mathrm{C}_{13} \mathrm{ik}+\mathrm{C}_{11} \delta \mathrm{R}_{5}-\overline{\alpha_{1}} \mathrm{Q}_{5} \quad \mathrm{~T}_{5}=\mathrm{C}_{13} \mathrm{ik}+\mathrm{C}_{11} \in \mathrm{R}_{6}-\overline{\alpha_{1}} \mathrm{Q}_{6} \quad \mathrm{~T}_{6}=\mathrm{C}_{13} \mathrm{ik}+\mathrm{C}_{11} \gamma \mathrm{R}_{7}-\overline{\alpha_{1}} \mathrm{Q}_{7} \\
& \mathrm{~V}_{1}=\mathrm{C}_{44}\left(-\delta+\mathrm{ikR}_{1}\right) \quad \mathrm{V}_{2}=\mathrm{C}_{44}\left(-\epsilon+\mathrm{ikR}_{2}\right) \quad \mathrm{V}_{3}=\mathrm{C}_{44}\left(-\gamma+\mathrm{ikR}_{3}\right) \\
& \mathrm{V}_{5}=\mathrm{C}_{44}\left(\delta+\mathrm{ikR}_{5}\right) \quad \mathrm{V}_{6}=\mathrm{C}_{44}\left(\epsilon+\mathrm{ikR}_{6}\right) \quad \mathrm{V}_{7}=\mathrm{C}_{44}\left(\gamma+\mathrm{ikR}_{7}\right) \\
& X_{1}=a_{3}+b_{3} i k Q_{1} \quad X_{2}=a_{3}+b_{3} i k Q_{2} \quad X_{3}=a_{3}+b_{3} i k Q_{3} \\
& \mathrm{X}_{5}=\mathrm{a}_{3}+\mathrm{b}_{3} \mathrm{ikQ}_{5} \quad \mathrm{X}_{6}=\mathrm{a}_{3}+\mathrm{b}_{3} \mathrm{ikQ}_{6} \quad \mathrm{X}_{7}=\mathrm{a}_{3}+\mathrm{b}_{3} \mathrm{ikQ}_{7} \\
& \mathrm{Z}_{1}=\mathrm{a}_{1} \mathrm{R}_{1}-\mathrm{b}_{1} \delta \mathrm{Q}_{1} \quad \mathrm{Z}_{2}=\mathrm{a}_{1} \mathrm{R}_{2}-\mathrm{b}_{1} \in \mathrm{Q}_{2} \quad \mathrm{Z}_{3}=\mathrm{a}_{1} \mathrm{R}_{3}-\mathrm{b}_{1} \gamma \mathrm{Q}_{3} \\
& \mathrm{Z}_{5}=\mathrm{a}_{1} \mathrm{R}_{5}+\mathrm{b}_{1} \delta \mathrm{Q}_{5} \quad \mathrm{Z}_{6}=\mathrm{a}_{1} \mathrm{R}_{6}+\mathrm{b}_{1} \in \mathrm{Q}_{6} \quad \mathrm{Z}_{7}=\mathrm{a}_{1} \mathrm{R}_{7}+\mathrm{b}_{1} \gamma \mathrm{Q}_{7} \\
& \mathrm{~S}_{4}=0 \quad \mathrm{~S}_{8}=0 \quad \mathrm{U}_{4}=-\mathrm{C}_{66} v \quad \mathrm{U}_{8}=\mathrm{C}_{66} v \\
& \mathrm{~W}_{4}=\mathrm{C}_{44} \mathrm{ik} \quad \mathrm{W}_{8}=\mathrm{C}_{44} \mathrm{ik} \quad \mathrm{Y}_{4}=\mathrm{a}_{1} \quad \mathrm{Y}_{8}=\mathrm{a}_{1}
\end{aligned}
$$

The definition for $a_{1}$ and $b_{1}, l=1,3$, can be found in Appendix $I$.

Alternately, we can also let $\mathrm{u}_{\mathrm{z}}$ instead of $\mathrm{u}_{\mathrm{x}}$ be the denominator of the ratios. In this case, the first and third rows of the matrix in Eq. (28) are exchanged. In addition, Eq. (30) should be revised accordingly. It should be pointed out that this is a better alternative when the amplitude of $u_{x}$ is small or vanishing, which will occur when $\mathrm{k}$ or $\phi$ is small.

\section{Propagation of Plane Waves Within an Arbitrary Plane in TIPM with $\boldsymbol{x}$ being the Axis of Symmetry}

of motion is given by

This is a three dimensional problem involving $\mathrm{u}_{\mathrm{x}}, \mathrm{u}_{\mathrm{y}}, \mathrm{u}_{\mathrm{z}}$ and $\mathrm{p}$. The equation 


$$
\begin{gathered}
\frac{\partial \tau_{x x}}{\partial x}+\frac{\partial \tau_{x y}}{\partial y}+\frac{\partial \tau_{x z}}{\partial z}-\overline{\alpha_{3}} \frac{\partial p}{\partial x}=\overline{\rho_{3}} \frac{\partial^{2} u_{x}}{\partial t^{2}} \\
\frac{\partial \tau_{x y}}{\partial x}+\frac{\partial \tau_{y y}}{\partial y}+\frac{\partial \tau_{y z}}{\partial z}-\overline{\alpha_{1}} \frac{\partial p}{\partial y}=\overline{\rho_{1}} \frac{\partial^{2} u_{y}}{\partial t^{2}} \\
\frac{\partial \tau_{x z}}{\partial x}+\frac{\partial \tau_{y z}}{\partial y}+\frac{\partial \tau_{z z}}{\partial z}-\bar{\alpha}_{1} \frac{\partial p}{\partial z}=\overline{\rho_{1}} \frac{\partial^{2} u_{z}}{\partial t^{2}} \\
-\bar{\alpha}_{3} \frac{\partial u_{x}}{\partial x}-\bar{\alpha}_{1} \frac{\partial u_{y}}{\partial y}-\overline{\alpha_{1}} \frac{\partial u_{z}}{\partial z}+\left[\theta_{3} \frac{\partial^{2}}{\partial x^{2}}+\theta_{1} \frac{\partial^{2}}{\partial y^{2}}+\theta_{1} \frac{\partial^{2}}{\partial z^{2}}-\beta\right] p=0
\end{gathered}
$$

which can be rewritten as

$$
\begin{array}{r}
{\left[C_{33} \frac{\partial^{2}}{\partial x^{2}}+C_{44}\left(\frac{\partial^{2}}{\partial y^{2}}+\frac{\partial^{2}}{\partial z^{2}}\right)+\omega^{2} \overline{\rho_{3}}\right] u_{x}+\left(C_{13}+C_{44}\right)\left(\frac{\partial^{2} u_{z}}{\partial x \partial z}+\frac{\partial^{2} u_{y}}{\partial x \partial y}\right)-\overline{\alpha_{3}} \frac{\partial p}{\partial x}=0} \\
\left(C_{13}+C_{44}\right) \frac{\partial^{2} u_{x}}{\partial x \partial y}+\left(C_{66}+C_{12}\right) \frac{\partial^{2} u_{z}}{\partial y \partial z}+\left[C_{44} \frac{\partial^{2}}{\partial x^{2}}+C_{11} \frac{\partial^{2}}{\partial y^{2}}+C_{66} \frac{\partial^{2}}{\partial z^{2}}+\omega^{2} \overline{\rho_{1}}\right] u_{y}-\overline{\alpha_{1}} \frac{\partial p}{\partial y}=0 \\
\left(C_{13}+C_{44}\right) \frac{\partial^{2} u_{x}}{\partial x \partial z}+\left(C_{12}+C_{66}\right) \frac{\partial^{2} u_{y}}{\partial y \partial z}+\left[C_{44} \frac{\partial^{2}}{\partial x^{2}}+C_{66} \frac{\partial^{2}}{\partial y^{2}}+C_{11} \frac{\partial^{2}}{\partial z^{2}}+\omega^{2} \overline{\rho_{1}}\right] u_{z}-\overline{\alpha_{1}} \frac{\partial p}{\partial z}=0 \\
-\overline{\alpha_{3}} \frac{\partial u_{x}}{\partial x}-\overline{\alpha_{1}} \frac{\partial u_{y}}{\partial y}-\overline{\alpha_{1}} \frac{\partial u_{z}}{\partial z}+\left[\theta_{3} \frac{\partial^{2}}{\partial x^{2}}+\theta_{1} \frac{\partial^{2}}{\partial y^{2}}+\theta_{1} \frac{\partial^{2}}{\partial z^{2}}-\beta\right] p=0
\end{array}
$$

Consider plane waves of the form

$$
\left\{\begin{array}{llll}
U_{x} & U_{y} & U_{z} & P
\end{array}\right\}^{T}=\left\{\begin{array}{lllll}
u_{x} & u_{y} & u_{z} & p
\end{array}\right\}^{T} \exp \left[i\left(\xi_{1} x+\xi_{2} y+\zeta z-c t\right)\right]
$$

Now that the incident wave is no longer in the $x-z$ plane, the roots of Eq. (25) are associated with wavenumber in the direction along the intersecting line formed by the plane of the incident ray and $x$-axis with the $y-z$ plane. However, owing to the isotropic nature of the $y-z$ plane, it can be shown that $\zeta^{2}$ in this section may be obtained from $\zeta^{2}$ solved from Eq. (25) by adding a term $\left(-\xi_{2}{ }^{2}\right)$. Similarly, Eq. (23) must be generalized to the following form

$$
c^{6}+c^{4} \omega^{2} h_{2}(\omega, \phi, \theta)+c^{2} \omega^{4} h_{1}(\omega, \phi, \theta)+\omega^{6} h_{0}(\omega, \phi, \theta)=0
$$

where $\theta$ is the azimuth angle. Coefficients of Eq. (36a) are given in Appendix $\Pi$.

Substituting Eq. (36) into Eq. (35) yields 


$$
\begin{aligned}
& {\left[\begin{array}{cccc}
\mathrm{C}_{33} \xi_{1}^{2}+\mathrm{C}_{44}\left(\xi_{2}^{2}+\zeta^{2}\right)-\omega^{2} \rho_{3} & \left(\mathrm{C}_{13}+\mathrm{C}_{44}\right) \xi_{1} \xi_{2} & \left(\mathrm{C}_{13}+\mathrm{C}_{44}\right) \xi_{1} \zeta & \mathrm{i} \xi_{1} \overline{\alpha_{3}} \\
\left(\mathrm{C}_{13}+\mathrm{C}_{44}\right) \xi_{1} \xi_{2} & \mathrm{C}_{44} \xi_{1}^{2}+\mathrm{C}_{11} \xi_{2}^{2}+\mathrm{C}_{66} \zeta^{2}-\omega^{2} \bar{\rho}_{1} & \left(\mathrm{C}_{12}+\mathrm{C}_{66}\right) \xi_{2} \zeta & \mathrm{i} \xi_{2} \overline{\alpha_{1}} \\
\left(\mathrm{C}_{13}+\mathrm{C}_{44}\right) \xi_{1} \zeta & \left(\mathrm{C}_{12}+\mathrm{C}_{66}\right) \xi_{2} \zeta & \mathrm{C}_{44} \xi_{1}^{2}+\mathrm{C}_{66} \xi_{2}^{2}+\mathrm{C}_{11} \zeta^{2}-\omega^{2} \overline{\rho_{1}} & \mathrm{i} \zeta \overline{\alpha_{1}} \\
\mathrm{i} \xi_{1} \overline{\alpha_{3}} & \mathrm{i} \xi_{2} \overline{\alpha_{1}} & \mathrm{i} \zeta \overline{\alpha_{1}} & \theta_{3} \xi_{1}^{2}+\theta_{1}\left(\xi_{2}^{2}+\zeta^{2}\right)+\beta
\end{array}\right]} \\
& x\left\{\begin{array}{llll}
u_{x} & u_{y} & u_{z} & p
\end{array}\right\}^{T}=\left\{\begin{array}{llll}
0 & 0 & 0 & 0
\end{array}\right\}^{T}
\end{aligned}
$$

Accordingly, $u_{x}, u_{y}, u_{z}$ and $p$ may be expressed in terms of eight wave coefficients:

$$
\left\{\begin{array}{l}
u_{x} \\
u_{y} \\
u_{z} \\
p
\end{array}\right\}=\left[\begin{array}{cccccccc}
1 & 1 & 1 & 0 & 1 & 1 & 1 & 0 \\
\kappa_{1} R_{1} & \kappa_{2} R_{2} & \kappa_{3} R_{3} & 1 & \kappa_{5} R_{5} & \kappa_{6} R_{6} & \kappa_{7} R_{7} & 1 \\
R_{1} & R_{2} & R_{3} & R_{4} & R_{5} & R_{6} & R_{7} & R_{8} \\
Q_{1} & Q_{2} & Q_{3} & 0 & Q_{5} & Q_{6} & Q_{7} & 0
\end{array}\right]\left\{\begin{array}{l}
A_{1} e^{-\delta z} \\
A_{2} e^{-\epsilon z} \\
A_{3} e^{-\gamma z} \\
A_{4} e^{-v z} \\
A_{5} e^{\delta z} \\
A_{6} e^{\epsilon z} \\
A_{7} e^{\gamma z} \\
A_{8} e^{v z}
\end{array}\right\} e^{i\left(\xi_{1} x+\xi_{2} y\right)}
$$

In Eq. (40) $\kappa_{j}, j=1,2,3,5,6,7$ are the ratio $u_{y} / u_{z}$ for fast and slow quasi-P waves and quasi-SV wave. $R_{4}, R_{8}$ are the ratio $u_{z} / u_{y}$ for the $S H$ wave, respectively. Because of the symmetry of the elastic and permeable properties in the $y$ - $z$ plane, we have

$$
\begin{array}{lll}
\kappa_{1}=-i \frac{\xi_{2}}{\delta} & \kappa_{2}=-i \frac{\xi_{2}}{\epsilon} \quad \kappa_{3}=-i \frac{\xi_{2}}{\gamma} \\
\kappa_{5}=-i \frac{\xi_{2}}{\delta} & \kappa_{6}=i \frac{\xi_{2}}{\epsilon} \quad \kappa_{1}=i \frac{\xi_{2}}{\gamma} \\
\mathrm{R}_{4}=-\frac{v}{i \xi_{2}} & \mathrm{R}_{8}=\frac{v}{i \xi_{2}}
\end{array}
$$


Coefficients $\left\{R_{i}, Q_{i}\right\}, i=1,2,3,5,6,7$ in Eq. (38) represent $\left\{u_{z} / u_{y}, p / u_{x}\right\}$. They can be obtained from (37), dividing the two sides of each equation by $u_{x}$ and substituting $\zeta$ by $i \delta, i \epsilon, i \gamma,-i \delta,-i \epsilon$, and -i $\gamma$, respectively.

$$
\mathrm{R}_{\mathrm{i}}=\frac{\Delta_{\mathrm{R}}}{\Delta} \quad \mathrm{Q}_{\mathrm{i}}=\frac{\Delta_{\mathrm{Q}}}{\Delta} \quad \mathrm{i}=1,2,3,5,6,7
$$

where

$$
\begin{aligned}
& \Delta=a_{30} a_{44}-a_{34} a_{40} \\
& \Delta_{R}=-a_{31} a_{44}+a_{41} a_{34} \\
& \Delta_{Q}=-a_{41} a_{30}+a_{31} a_{40} \\
& a_{30}=a_{33}+\kappa_{j} a_{32} \\
& a_{40}=a_{43}+\kappa_{j} a_{42} \\
& a_{31}=\left(C_{13}+C_{44}\right) \xi_{1} \zeta \\
& a_{32}=\left(C_{12}+C_{66}\right) \xi_{2} \zeta \\
& a_{33}=C_{44} \xi_{2}^{2}+C_{66} \xi_{2}^{2}+C_{11} \zeta^{2}-\omega^{2} \overline{\rho_{1}} \\
& a_{34}=i \zeta \alpha_{1} \\
& a_{41}=i \xi_{1} \\
& a_{42}=i \xi_{2} \\
& a_{43}=i \zeta \overline{\alpha_{1}} \\
& a_{44}=\theta_{3} \xi_{1}^{2}+\theta_{1}\left(\xi_{2}^{2}+\zeta^{2}\right)+\beta
\end{aligned}
$$

and $j=1,2,3,5,6,7$.

8. Propagation of Plane Waves Within an Arbitrary Plane in LTIPM with $\boldsymbol{x}$ being the Axis of Symmetry and Interfaces Parallel to the $x-y$ Plane

Denote the incident angle by $\phi$, azimuth angle by $\theta$, the angle between the incident wave and the symmetry axis of the transverse isotropy $(x)$ by $\phi_{0}$, and the angle between the plane of the incident ray and symmetry axis with $\mathrm{z}$-axis by $\psi$. Clearly

$$
\begin{aligned}
& \xi_{1}=\mathrm{k}_{0} \sin \phi \cos \theta \\
& \xi_{2}=\mathrm{k}_{0} \sin \phi \sin \theta
\end{aligned}
$$

by

It can also be shown that the relationships among these four angles are given

$$
\cos \phi_{0}=\sin \phi \cos \theta
$$


and

$$
\sin \psi=\sin \phi \sin \theta
$$

It should be noted that angle $\phi_{0}$ can be used to replace $\phi$ in Eq. (11) to obtain the phase velocities which are identical to the result given by Eq. (23a).

The general expressions of all stress and volumetric flux components in a three-dimensional case are given by

$$
\begin{aligned}
& \left\{\begin{array}{c}
\tau_{x x} \\
\tau_{y y} \\
\tau_{z z}
\end{array}\right\}=\left[\begin{array}{lll}
C_{33} & C_{13} & C_{13} \\
C_{13} & C_{11} & C_{12} \\
C_{13} & C_{12} & C_{11}
\end{array}\right]\left\{\begin{array}{l}
\frac{\partial u_{x}}{\partial x} \\
\frac{\partial u_{y}}{\partial y} \\
\frac{\partial u_{z}}{\partial z}
\end{array}\right\}-p\left\{\begin{array}{l}
\overline{\alpha_{3}} \\
\overline{\alpha_{1}} \\
\frac{\alpha_{1}}{\partial y}
\end{array}\right\} \\
& \left\{\begin{array}{c}
\tau_{y z} \\
\tau_{x z} \\
\tau_{x y}
\end{array}\right\}=\left[\begin{array}{ccc}
C_{66} & 0 & 0 \\
0 & C_{44} & 0 \\
0 & 0 & C_{44}
\end{array}\right]\left\{\begin{array}{l}
\frac{\partial u_{y}}{\partial z}+\frac{\partial u_{z}}{\partial y} \\
\frac{\partial u_{x}}{\partial z}+\frac{\partial u_{z}}{\partial x} \\
\frac{\partial u_{x}}{\partial y}+\frac{\partial u_{y}}{\partial x}
\end{array}\right\} \\
& \left\{\begin{array}{l}
w_{x} \\
w_{y} \\
w_{z}
\end{array}\right\}=\left\{\begin{array}{l}
a_{3} u_{x} \\
a_{1} u_{y} \\
a_{1} u_{z}
\end{array}\right\}+\left\{\begin{array}{l}
b_{3} \frac{\partial p}{\partial x} \\
b_{1} \frac{\partial p}{\partial y} \\
b_{1} \frac{\partial p}{\partial z}
\end{array}\right\}
\end{aligned}
$$

Substituting Eq. (38) into Eq. (45) provides the following: 


$$
\left\{\begin{array}{c}
\tau_{x x} \\
\tau_{y y} \\
\tau_{z z} \\
\tau_{y z} \\
\tau_{x z} \\
\tau_{x y} \\
w_{x} \\
w_{y} \\
w_{z}
\end{array}\right\}=\left[\begin{array}{cccccccc}
P_{1} & P_{2} & P_{3} & P_{4} & P_{5} & P_{6} & P_{7} & P_{8} \\
G_{1} & G_{2} & G_{3} & G_{4} & G_{5} & G_{6} & G_{7} & G_{8} \\
T_{1} & T_{2} & T_{3} & T_{4} & T_{5} & T_{6} & T_{7} & T_{8} \\
U_{1} & U_{2} & U_{3} & U_{4} & U_{5} & U_{6} & U_{7} & U_{8} \\
V_{1} & V_{2} & V_{3} & V_{4} & V_{5} & V_{6} & V_{7} & V_{8} \\
W_{1} & W_{2} & W_{3} & W_{4} & W_{5} & W_{6} & W_{7} & W_{8} \\
X_{1} & X_{2} & X_{3} & 0 & X_{5} & X_{6} & X_{7} & 0 \\
Y_{1} & Y_{2} & Y_{3} & Y_{4} & Y_{5} & Y_{6} & Y_{7} & Y_{8} \\
Z_{1} & Z_{2} & Z_{3} & Z_{4} & Z_{5} & Z_{6} & Z_{7} & Z_{8}
\end{array}\right]\left\{\begin{array}{l}
A_{1} e^{-\delta z} \\
A_{2} e^{-\epsilon z} \\
A_{3} e^{-\gamma z} \\
A_{4} e^{-v z} \\
A_{5} e^{\delta z} \\
A_{6} e^{\epsilon z} \\
A_{7} e^{\gamma z} \\
A_{8} e^{v z}
\end{array}\right\} e^{i\left(\xi_{1} x+x_{2} y\right)}
$$

where

$$
\begin{array}{cc}
P_{1}=C_{33} i \xi_{1}+C_{13}\left(i \xi_{2} \kappa_{1}-\delta\right) R_{1}-\overline{\alpha_{3}} Q_{1} & P_{2}=C_{33} i \xi_{1}+C_{13}\left(i \xi_{2} \kappa_{2}-\epsilon\right) R_{2} \overline{\alpha_{3}} Q_{2} \\
P_{3}=C_{33} i \xi_{1}+C_{13}\left(i \xi_{2} \kappa_{3}-\gamma\right) R_{3}-\overline{\alpha_{3}} Q_{3} & P_{5}=C_{33} i \xi_{1}+C_{13}\left(i \xi_{2} \kappa_{5}+\delta\right) R_{5}-\overline{\alpha_{3}} Q_{5} \\
P_{6}=C_{33} i \xi_{1}+C_{13}\left(i \xi_{2} \kappa_{6}+\epsilon\right) R_{6}-\overline{\alpha_{3}} Q_{6} & P_{7}=C_{33} i \xi_{1}+C_{13}\left(i \xi_{2} \kappa_{7}+\gamma\right) R_{7}-\overline{\alpha_{3}} Q_{7} \\
G_{1}=C_{13} i \xi_{1}+\left(C_{11} i \xi_{2} \kappa_{1}-C_{12} \delta\right) R_{1}-\overline{\alpha_{1}} Q_{1} & G_{2}=C_{13} i \xi_{1}+\left(C_{11} i \xi_{2} \kappa_{2}-C_{12} \epsilon\right) R_{2}-\overline{\alpha_{1}} Q_{2} \\
G_{3}=C_{13} i \xi_{1}+\left(C_{11} i \xi_{2} \kappa_{3}-C_{12} \gamma\right) R_{3}-\overline{\alpha_{1}} Q_{3} & G_{5}=C_{13} i \xi_{1}+\left(C_{11} i \xi_{2} \kappa_{5}+C_{12} \delta\right) R_{5}-\overline{\alpha_{1}} Q_{5} \\
G_{6}=C_{13} i \xi_{1}+\left(C_{11} i \xi_{2} \kappa_{6}+C_{12} \epsilon\right) R_{6}-\overline{\alpha_{1}} Q_{6} & G_{7}=C_{13} i \xi_{1}+\left(C_{11} i \xi_{2} \kappa_{7}+C_{12} \gamma\right) R_{7}-\overline{\alpha_{1}} Q_{7} \\
T_{1}=C_{13} i \xi_{1}+\left(C_{12} i \xi_{2} \kappa_{1}-C_{11} \delta\right) R_{1}-\overline{\alpha_{1}} Q_{1} & T_{2}=C_{13} i \xi_{1}+\left(C_{12} i \xi_{2} \kappa_{2}-C_{11} \epsilon\right) R_{2}-\overline{\alpha_{1}} Q_{2} \\
T_{3}=C_{13} i \xi_{1}+\left(C_{12} i \xi_{2} \kappa_{3}-C_{11} \gamma\right) R_{3}-\overline{\alpha_{1}} Q_{3} & T_{5}=C_{13} i \xi_{1}+\left(C_{12} i \xi_{2} \kappa_{5}+C_{11} \delta\right) R_{5}-\overline{\alpha_{1}} Q_{5} \\
T_{6}=C_{13} i \xi_{1}+\left(C_{12} i \xi_{2} \kappa_{6}+C_{11} \epsilon\right) R_{6}-\overline{\alpha_{1}} Q_{6} & T_{7}=C_{13} i \xi_{1}+\left(C_{12} i \xi_{2} \kappa_{7}+C_{11} \gamma\right) R_{7}-\overline{\alpha_{1}} Q_{7}
\end{array}
$$




$$
\begin{array}{ccc}
\mathrm{U}_{1}=2 \mathrm{C}_{66} \mathrm{i} \xi_{2} \mathrm{R}_{1} & \mathrm{U}_{2}=2 \mathrm{C}_{66} \mathrm{i} \xi_{2} \mathrm{R}_{2} & \mathrm{U}_{3}=2 \mathrm{C}_{66} \mathrm{i} \xi_{2} \mathrm{R}_{3} \\
\mathrm{U}_{5}=2 \mathrm{C}_{66} \mathrm{i} \xi_{2} \mathrm{R}_{5} & \mathrm{U}_{6}=2 \mathrm{C}_{66} \mathrm{i} \xi_{2} \mathrm{R}_{6} & \mathrm{U}_{7}=2 \mathrm{C}_{66} \mathrm{i} \xi_{2} \mathrm{R}_{7} \\
\mathrm{~V}_{1}=\mathrm{C}_{44}\left(-\delta+\mathrm{i} \xi_{1} \mathrm{R}_{1}\right) & \mathrm{V}_{2}=\mathrm{C}_{44}\left(-\epsilon+\mathrm{i} \xi_{1} \mathrm{R}_{2}\right) & \mathrm{V}_{3}=\mathrm{C}_{44}\left(-\gamma+\mathrm{i} \xi_{1} \mathrm{R}_{3}\right) \\
\mathrm{V}_{5}=\mathrm{C}_{44}\left(\delta+\mathrm{i} \xi_{1} \mathrm{R}_{5}\right) & \mathrm{V}_{6}=\mathrm{C}_{44}\left(\epsilon+\mathrm{i} \xi_{1} \mathrm{R}_{6}\right) & \mathrm{V}_{7}=\mathrm{C}_{44}\left(\gamma+\mathrm{i} \xi_{1} \mathrm{R}_{7}\right) \\
\mathrm{W}_{1}=\mathrm{C}_{44} \mathrm{i}\left(\xi_{2}+\xi_{1} \kappa_{1} \mathrm{R}_{1}\right) & \mathrm{W}_{2}=\mathrm{C}_{44} \mathrm{i}\left(\xi_{2}+\xi_{1} \kappa_{2} \mathrm{R}_{2}\right) & \mathrm{W}_{3}=\mathrm{C}_{44} \mathrm{i}\left(\xi_{2}+\xi_{1} \kappa_{3} \mathrm{R}_{3}\right) \\
\mathrm{W}_{5}=\mathrm{C}_{44} \mathrm{i}\left(\xi_{2}+\xi_{1} \kappa_{5} \mathrm{R}_{5}\right) & \mathrm{W}_{6}=\mathrm{C}_{44} \mathrm{i}\left(\xi_{2}+\xi_{1} \kappa_{6} \mathrm{R}_{6}\right) & \mathrm{W}_{7}=\mathrm{C}_{44} \mathrm{i}\left(\xi_{2}+\xi_{1} \kappa_{7} \mathrm{R}_{7}\right) \\
\mathrm{X}_{1}=\mathrm{a}_{3}+\mathrm{b}_{3} \mathrm{i} \xi_{1} \mathrm{Q}_{1} & \mathrm{X}_{2}=\mathrm{a}_{3}+\mathrm{b}_{3} \mathrm{i} \xi_{1} \mathrm{Q}_{2} & \mathrm{X}_{3}=\mathrm{a}_{3}+\mathrm{b}_{3} \mathrm{i} \xi_{1} \mathrm{Q}_{3} \\
\mathrm{X}_{5}=\mathrm{a}_{3}+\mathrm{b}_{3} \mathrm{i} \xi_{1} \mathrm{Q}_{5} & \mathrm{X}_{6}=\mathrm{a}_{3}+\mathrm{b}_{3} \mathrm{i} \xi_{1} \mathrm{Q}_{6} & \mathrm{X}_{7}=\mathrm{a}_{3}+\mathrm{b}_{3} \mathrm{i} \xi_{1} \mathrm{Q}_{7} \\
\mathrm{Y}_{1}=\mathrm{a}_{1} \mathrm{~K}_{1} \mathrm{R}_{1}+\mathrm{b}_{1} \mathrm{i} \xi_{2} \mathrm{Q}_{1} & \mathrm{Y}_{2}=\mathrm{a}_{1} \kappa_{2} \mathrm{R}_{2}+\mathrm{b}_{1} \mathrm{i} \xi_{2} \mathrm{Q}_{2} & \mathrm{Y}_{3}=\mathrm{a}_{1} \kappa_{3} \mathrm{R}_{3}+\mathrm{b}_{1} \mathrm{i} \xi_{2} \mathrm{Q}_{3} \\
\mathrm{Y}_{5}=\mathrm{a}_{1} \kappa_{5} \mathrm{R}_{5}+\mathrm{b}_{1} \mathrm{i} \xi_{2} \mathrm{Q}_{5} & \mathrm{Y}_{6}=\mathrm{a}_{1} \kappa_{6} \mathrm{R}_{6}+\mathrm{b}_{1} \mathrm{i} \xi_{2} \mathrm{Q}_{6} & \mathrm{Y}_{7}=\mathrm{a}_{1} \kappa_{7} \mathrm{R}_{7}+\mathrm{b}_{1} \mathrm{i} \xi_{2} \mathrm{Q}_{7} \\
\mathrm{Z}_{1}=\mathrm{a}_{1} \mathrm{R}_{1}-\mathrm{b}_{1} \delta \mathrm{Q}_{1} & \mathrm{Z}_{2}=\mathrm{a}_{1} \mathrm{R}_{2}-\mathrm{b}_{1} \in \mathrm{Q}_{2} & \mathrm{Z}_{3}=\mathrm{a}_{1} \mathrm{R}_{3}-\mathrm{b}_{1} \gamma \mathrm{Q}_{3} \\
\mathrm{Z}_{5}=\mathrm{a}_{1} \mathrm{R}_{5}+\mathrm{b}_{1} \delta \mathrm{Q}_{5} & \mathrm{Z}_{6}=\mathrm{a}_{1} \mathrm{R}_{6}+\mathrm{b}_{1} \in \mathrm{Q}_{6} & \mathrm{Z}_{7}=\mathrm{a}_{1} \mathrm{R}_{7}+\mathrm{b}_{1} \gamma \mathrm{Q}_{7}
\end{array}
$$

and

$$
\begin{array}{cc}
\mathrm{P}_{4}=\mathrm{C}_{13}\left(\mathrm{i} \xi_{2}-v R_{4}\right) & \mathrm{P}_{8}=\mathrm{C}_{13}\left(\mathrm{i} \xi_{2}+v R_{8}\right) \\
\mathrm{G}_{4}=\mathrm{C}_{11} \mathrm{i} \xi_{2}-\mathrm{C}_{12} \nu R_{4} & \mathrm{G}_{8}=\mathrm{C}_{11} \mathrm{i} \xi_{2}+\mathrm{C}_{12} \nu \mathrm{R}_{8} \\
\mathrm{~T}_{4}=\mathrm{C}_{12} \mathrm{i} \xi_{2}-\mathrm{C}_{11} v \mathrm{R}_{4} & \mathrm{~T}_{8}=\mathrm{C}_{12} \mathrm{i} \xi_{2}+\mathrm{C}_{11} v \mathrm{R}_{8} \\
\mathrm{U}_{4}=\mathrm{C}_{66}\left(-v+\mathrm{i} \xi_{2} \mathrm{R}_{4}\right) & \mathrm{U}_{8}=\mathrm{C}_{66}\left(v+\mathrm{i} \xi_{2} \mathrm{R}_{8}\right) \\
\mathrm{V}_{4}=\mathrm{C}_{44} \mathrm{i} \xi_{1} \mathrm{R}_{4} & \mathrm{~V}_{8}=\mathrm{C}_{44} \mathrm{i} \xi_{1} \mathrm{R}_{8} \\
\mathrm{~W}_{4}=\mathrm{C}_{44} \mathrm{i} \xi_{1} & \mathrm{~W}_{8}=\mathrm{C}_{44} \mathrm{i} \xi_{1} \\
\mathrm{Y}_{4}=\mathrm{a}_{1} & \mathrm{Y}_{8}=\mathrm{a}_{1} \\
\mathrm{Z}_{4}=\mathrm{a}_{1} \mathrm{R}_{4} & \mathrm{Z}_{8}=\mathrm{a}_{1} \mathrm{R}_{8}
\end{array}
$$

It is noted that the boundary conditions for waves in LTIPM require the use of $\left\{u_{x}, u_{y}, u_{z}, p, \tau_{x z}, \tau_{x z}, \tau_{x z}, w_{z}\right\}$. 


\section{Response of the LTIPM to Incident Plane Waves}

The above analysis provides the formulation for the wave motion in a layer of the LTIPM in terms of its eight coefficients. The response of the entire layered medium to incident plane waves poses a boundary value problem in which the following equation is satisfied:

$$
\begin{aligned}
& \left\{u_{x}^{(n+1)} u_{y}^{(n+1)} \quad u_{z}^{(n+1)} p^{(n+1)} \tau_{x z}^{(n+1)} \tau_{y z}^{(n+1)} \tau_{z z}^{(n+1)} w_{z}^{(n+1)}\right\}^{T}
\end{aligned}
$$

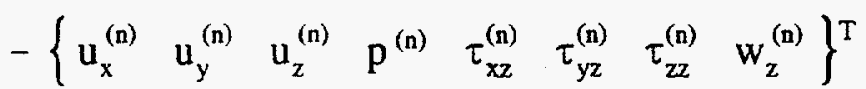

$$
\begin{aligned}
& =\left\{\begin{array}{llllllll}
0 & 0 & 0 & 0 & 0 & 0 & 0 & 0
\end{array}\right\}^{\mathrm{T}}
\end{aligned}
$$

where $n=1,2, \ldots N$ are labels of the layers. The matrix corresponding to these components is denoted by $[m]$.

Finally, this boundary value problem can be reduced to a linear system of equations (see Ref. [2])

$$
[\mathrm{M}]\{\mathrm{A}\}=\{\mathrm{F}\}
$$

where $\{A\}$ contains unknown coefficients $A_{j} j=1,2 \ldots 8$, from all layers and/or half spaces. Matrix $[M]$ is assembled from $[m]$ of all layers and/or half spaces. The right hand side vector, $\{F\}$, is given and is dependent upon the frequency, property of the source medium, type of indecent wave, and incident and azimuth angles.

\section{Scaling the Incident Plane Wave}

In the right hand side vector $\{F\}$ of Eq. (51), the discontinuity, if any, of the displacement and stress components at the top interface due to the incident wave must be provided, using the expression in Eq. (17) for a given non-zero $A_{1}$ (fast $P$ wave), $A_{3}$ (quasi-SV wave) or $A_{4}$ (quasi-SH wave) and vanishing $A_{2}, A_{5}, A_{6}, A_{7}$ and $A_{8}$. Clearly, the result of Eq. (51) is proportional to these constants. It is then necessary to scale the incident wave, i.e., define the displacement-stress field in a unbounded medium caused by the incident wave with a unit amplitude. To this end, each column of the matrices in Eqs. (38) and (46) can be multiplied by a specific factor such that when the medium is reduced to the isotropic case, the result should be identical to that for an isotropic case subjected to incident fast $P$, quasi-SV, or quasi-SH wave with a unit amplitude potential. The simplest way to accomplish this is to use factors $i \xi_{1}, i \xi_{1}, i \xi_{1} q_{13} / q_{33}$, and 1 for columns $1,2,3$, and 4 respectively, where

$$
\begin{aligned}
& q_{13}=\frac{C_{13}+C_{44}}{\rho}\left(-\gamma^{2}+\xi_{2}^{2}\right) \\
& q_{33}=\omega^{2}-\frac{C_{33}}{\rho} \xi_{1}^{2}-\frac{C_{44}}{\rho}\left(-\gamma^{2}+\xi_{2}^{2}\right)
\end{aligned}
$$


The same steps are also applied to columns 5,6,7 and 8. It is noted that under the isotropic condition and for $\theta=0, q_{13} / q_{33}=-\zeta_{3}{ }^{2} / \xi_{1}{ }^{2}$. As a result, it can be shown that the matrices in Eqs. (38) and (46) will be consistent with the standard form in the isotropic case when $\theta=0$.

\section{References}

[1] J. O. Parra, A Plane Wave Solution for the Anisotropic Poroelastic Wave Equation, Internal Report, Southwest Research Institute, January, 1993.

[2] P.-C. Xu, A. K. Mal and Y. Bar-Cohen, "Inversion of Leaky Lamb Wave Data to Determine Cohesive Properties of Bonds", Int. J. Engng. Sci., Vol. 28, No. 4,pp. 331-346, 1990.

[3] M. Kazi-Azoual, Green's functions in an infinite transversely isotropic saturated poroelastic medium, J. Acoust. Soc. Am., 84, 1883-1889, 1988. 


\section{APPENDIX I}

Coefficients of Eq. (11)

$$
\mathrm{f}_{0}(\omega)=\left[\frac{\theta_{1}}{\beta} \sin ^{2} \phi+\frac{\theta_{3}}{\beta} \cos ^{2} \phi\right] \mathrm{L}(\omega)
$$

$$
\begin{aligned}
& \mathrm{f}_{1}(\omega)=\mathrm{L}(\omega)-\frac{\theta_{1}}{\beta}\left[\frac{1}{\mathrm{k}_{11}^{2}}+\frac{1}{\mathrm{k}_{44 \mathrm{v}}^{2}}\right] \sin ^{4} \phi-\frac{\theta_{3}}{\beta}\left[\frac{1}{\mathrm{k}_{44 \mathrm{~h}}^{2}}+\frac{1}{\mathrm{k}_{33}^{2}}\right] \cos ^{4} \phi \\
& -\frac{\theta_{1}}{\beta}\left[\frac{1}{\mathrm{k}_{44 \mathrm{~h}}^{2}}+\frac{1}{\mathrm{k}_{33}^{2}}\right] \sin ^{2} \phi \cos ^{2} \phi-\frac{\theta_{3}}{\beta}\left[\frac{1}{\mathrm{k}_{11}^{2}}+\frac{1}{\mathrm{k}_{44 \mathrm{v}}^{2}}\right] \sin ^{2} \phi \cos ^{2} \phi \\
& +\frac{1}{\mathrm{k}_{11}^{2}} \frac{\theta_{1}}{\beta}\left[\frac{1}{\mathrm{k}_{44 \mathrm{v}}^{2}}-\frac{1}{\mathrm{k}_{\mathrm{b}}^{2}}\right] \frac{2 \overline{\alpha_{1} \alpha_{3}}}{\mathrm{C}_{11} \theta_{1}} \sin ^{2} \phi \cos ^{2} \phi \\
& +\frac{1}{\mathrm{k}_{11}^{2}} \frac{\theta_{1}}{\beta}\left[\sin ^{2} \phi \frac{1}{\mathrm{k}_{44 \mathrm{v}}^{2}}+\frac{\cos ^{2} \phi}{\mathrm{k}_{33}^{2}}\right] \frac{\overline{\alpha_{1}^{2}}}{\mathrm{C}_{11} \theta_{1}} \sin ^{2} \phi \\
& +\frac{1}{\mathrm{k}_{33}^{2}} \frac{\theta_{3}}{\beta}\left[\frac{\sin ^{2} \phi}{\mathrm{k}_{11}^{2}}+\frac{\cos ^{2} \phi}{\mathrm{k}_{44 \mathrm{~h}}^{2}}\right] \frac{{\overline{\alpha_{3}}}^{2}}{\mathrm{C}_{33} \theta_{3}} \cos ^{2} \phi
\end{aligned}
$$

$$
\begin{aligned}
& \mathrm{f}_{2}(\omega)=\frac{\theta_{1}}{\beta} \frac{1}{\mathrm{k}_{11}^{2} \mathrm{k}_{44 \mathrm{v}}^{2}}\left[\mathrm{k}_{11}^{2}\left(\mathrm{k}_{44 \mathrm{v}}^{2}-\frac{\beta}{\theta_{1}}\right)-\mathrm{k}_{44 \mathrm{v}}^{2}\left(\frac{\overline{\alpha_{1}^{2}}}{\theta_{1} \mathrm{C}_{11}}+\frac{\beta}{\theta_{1}}\right)\right] \sin ^{2} \phi \\
& +\frac{\theta_{3}}{\beta} \frac{1}{\mathrm{k}_{33}^{2} \mathrm{k}_{44 \mathrm{~h}}^{2}}\left[\mathrm{k}_{33}^{2}\left(\mathrm{k}_{44 \mathrm{~h}}^{2}-\frac{\beta}{\theta_{3}}\right)-\mathrm{k}_{44 \mathrm{~h}}^{2}\left(\frac{\overline{\alpha_{3}^{2}}}{\theta_{3} \mathrm{C}_{33}}+\frac{\beta}{\theta_{3}}\right)\right] \cos ^{2} \phi
\end{aligned}
$$

$$
\mathrm{L}(\omega)=\left[\frac{\sin ^{2} \phi}{\mathrm{k}_{1 \mathrm{l}}^{2}}+\frac{\cos ^{2} \phi}{\mathrm{k}_{44 \mathrm{~h}}^{2}}\right]\left[\frac{\sin ^{2} \phi}{\mathrm{k}_{44 \mathrm{v}}^{2}}+\frac{\cos ^{2} \phi}{\mathrm{k}_{33}^{2}}\right]-\left[\frac{1}{\mathrm{k}_{\mathrm{a}}^{2} \mathrm{k}_{\mathrm{b}}^{2}}+\frac{1}{\mathrm{k}_{44 \mathrm{~h}}^{2} \mathrm{k}_{44 \mathrm{v}}^{2}}-\frac{2}{\mathrm{k}_{44 \mathrm{~h}}^{2} \mathrm{k}_{\mathrm{b}}^{2}}\right] \cos ^{2} \phi \sin ^{2} \phi
$$




$$
\begin{aligned}
& \mathrm{k}_{11}^{2}=\frac{\overline{\rho_{1}} \omega^{2}}{\mathrm{C}_{11}} \quad \mathrm{k}_{44 \mathrm{~h}}^{2}=\frac{\overline{\rho_{1}} \omega^{2}}{\mathrm{C}_{44}} \quad \mathrm{k}_{\mathrm{a}}^{2}=\frac{\overline{\rho_{1}} \omega^{2}}{\mathrm{C}_{13}+2 \mathrm{C}_{44}} \\
& \mathrm{k}_{33}^{2}=\frac{\overline{\rho_{3}} \omega^{2}}{\mathrm{C}_{33}} \quad \mathrm{k}_{44 \mathrm{v}}^{2}=\frac{\overline{\rho_{3}} \omega^{2}}{\mathrm{C}_{44}} \quad \mathrm{k}_{\mathrm{b}}^{2}=\frac{\overline{\rho_{3}} \omega^{2}}{\mathrm{C}_{13}+2 \mathrm{C}_{44}} \\
& \overline{\rho_{1}}=\rho+\omega^{2} \rho_{\mathrm{f}}^{2} \theta_{1} \quad \overline{\rho_{3}}=\rho+\omega^{2} \rho_{\mathrm{f}}^{2} \theta_{3} \\
& \overline{\alpha_{1}}=\alpha_{1}+\omega^{2} \rho_{\mathrm{f}} \theta_{1} \quad \overline{\alpha_{3}}=\alpha_{3}+\omega^{2} \rho_{\mathrm{f}} \theta_{3} \\
& \theta_{1}=-\mathrm{K}_{1}(\omega) /(\mathrm{i} \omega) \quad \theta_{3}=-\mathrm{K}_{3}(\omega) /(\mathrm{i} \omega) \\
& a_{1}=\omega^{2} \rho_{f} \theta_{1} \quad a_{3}=\omega^{2} \rho_{f} \theta_{3} \\
& b_{1}=-\theta_{1} \quad b_{3}=-\theta_{3}
\end{aligned}
$$

where $K_{l}(\omega), l=1,3$ is defined in Ref. 1 .

$$
\begin{gathered}
R(q)=\frac{\left(C_{33}+C_{44}\right)\left(\theta_{1} k^{2}-\theta_{3} q^{2}+\beta\right)+\overline{\alpha_{1} \alpha_{3}}}{\left(-C_{44} k^{2}+C_{33} q^{2}+\omega^{2} \overline{\rho_{3}}\right)\left(\theta_{1} k^{2}-\theta_{3} q^{2}+\beta\right)+\overline{\alpha_{3} q^{2}}} \\
S(q)=\frac{\overline{\alpha_{1} k^{2}-\overline{\alpha_{3}} q^{2} k^{2} R(q)}}{\theta_{1} k^{2}-\theta_{3} q^{2}+\beta}
\end{gathered}
$$




\section{Coefficients of Eq. (36a)}

$$
\begin{aligned}
h_{0}(\omega, \phi, \theta) & =A_{6} \sin ^{6} \phi \cos ^{6} \theta+A_{4}\left(\sin ^{2} \phi \sin ^{2} \theta+\cos ^{2} \phi\right) \sin ^{4} \phi \cos ^{4} \theta \\
& +A_{2} \sin ^{2} \phi \cos ^{2} \theta\left(\sin ^{2} \phi \sin ^{2} \theta+\cos ^{2} \phi\right)^{2}+A_{0}\left(\sin ^{2} \phi \sin ^{2} \theta+\cos ^{2} \phi\right)^{3}
\end{aligned}
$$

where

$$
\begin{aligned}
& A_{6}=\frac{\overline{\theta_{3}}}{\beta} \frac{1}{\mathrm{k}_{33}^{2} \mathrm{k}_{44 \mathrm{~h}}^{2}} \\
& \mathrm{~A}_{4}=\frac{\overline{\theta_{1}}}{\beta} \frac{1}{\mathrm{k}_{33}^{2} \mathrm{k}_{44 \mathrm{~h}}^{2}}+\frac{\overline{\theta_{3}}}{\beta} \mathrm{W}(\omega) \\
& A_{2}=\frac{\overline{\theta_{3}}}{\beta} \frac{1}{k_{11}^{2} k_{44 v}^{2}}+\frac{\overline{\theta_{1}}}{\beta} W(\omega) \\
& A_{0}=\frac{\overline{\theta_{1}}}{\beta} \frac{1}{\mathrm{k}_{11}^{2} \mathrm{k}_{44 \mathrm{v}}^{2}} \\
& \mathrm{~W}(\omega)=\frac{1}{\mathrm{k}_{33}^{2} \mathrm{k}_{11}^{2}}-\frac{1}{\mathrm{k}_{\mathrm{a}}^{2} \mathrm{k}_{\mathrm{b}}^{2}}+\frac{2}{\mathrm{k}_{44 \mathrm{~h}}^{2} \mathrm{k}_{\mathrm{b}}^{2}} \\
& \mathrm{~h}_{1}(\omega, \phi, \theta)=\mathrm{B}_{4} \sin ^{4} \phi \cos ^{4} \theta+\mathrm{B}_{2} \sin ^{2} \phi \cos ^{2} \theta\left(\sin ^{2} \phi \sin ^{2} \theta+\cos ^{2} \phi\right) \\
& +\mathrm{B}_{0}\left(\sin ^{2} \phi \sin ^{2} \theta+\cos ^{2} \phi\right)^{2}
\end{aligned}
$$

where

$$
\begin{aligned}
\mathrm{B}_{4} & =\frac{1}{\mathrm{k}_{33}^{2} \mathrm{k}_{44 \mathrm{~h}}^{2}}+\frac{1}{\beta} \frac{\bar{\alpha}_{3}^{2}}{\mathrm{C}_{33}} \frac{1}{\mathrm{k}_{33}^{2} \mathrm{k}_{44 \mathrm{~h}}^{2}}-\frac{\bar{\theta}_{3}}{\beta}\left(\frac{1}{\mathrm{k}_{44 \mathrm{~h}}^{2}}+\frac{1}{\mathrm{k}_{33}^{2}}\right) \\
\mathrm{B}_{2} & =\frac{1}{\beta} \frac{\bar{\alpha}_{3}^{2}}{\mathrm{C}_{33}} \frac{1}{\mathrm{k}_{11}^{2} \mathrm{k}_{33}^{2}}+\frac{1}{\beta} \frac{\bar{\alpha}_{1}^{2}}{\mathrm{C}_{11} \mathrm{k}_{11}^{2} \mathrm{k}_{33}^{2}}+\frac{1}{\beta \mathrm{k}_{11}^{2}}\left(\frac{1}{\mathrm{k}_{44 \mathrm{v}}^{2}}-\frac{1}{\mathrm{k}_{\mathrm{b}}^{2}}\right) \frac{\alpha_{1} \bar{\alpha}_{3}+\bar{\alpha}_{1} \alpha_{3}}{\mathrm{C}_{11}} \\
& -\frac{\bar{\theta}_{1}}{\beta}\left(\frac{1}{\mathrm{k}_{33}^{2}}+\frac{1}{\mathrm{k}_{44 \mathrm{~h}}^{2}}\right)-\frac{\bar{\theta}_{3}}{\beta}\left(\frac{1}{\mathrm{k}_{11}^{2}}+\frac{1}{\mathrm{k}_{44 \mathrm{v}}^{2}}\right)+\mathrm{W}(\omega) \\
\mathrm{B}_{0} & =\frac{1}{\beta} \frac{\bar{\alpha}_{1}^{2}}{\mathrm{C}_{11} \mathrm{k}_{11}^{2} \mathrm{k}_{44 \mathrm{v}}^{2}}-\frac{1}{\beta}\left(\frac{1}{\mathrm{k}_{11}^{2}}+\frac{1}{\mathrm{k}_{44 \mathrm{v}}^{2}}\right)+\frac{1}{\mathrm{k}_{11}^{2} \mathrm{k}_{44 \mathrm{v}}^{2}}
\end{aligned}
$$




$$
h_{2}(\omega)=C_{2} \sin ^{2} \phi \cos ^{2} \theta+C_{0}\left(\sin ^{2} \phi \sin ^{2} \theta+\cos ^{2} \phi\right)
$$

where

$$
\begin{aligned}
& C_{2}=\frac{\bar{\theta}_{3}}{\beta} \frac{1}{k_{33}^{2} k_{44 \mathrm{~h}}^{2}}\left[k_{33}^{2}\left(k_{44 \mathrm{~h}}^{2}-\frac{\beta}{\bar{\theta}_{3}}\right)-k_{44 \mathrm{~h}}^{2}\left(\frac{\bar{\alpha}_{3}^{2}}{\mathrm{C}_{33} \theta_{3}}+\frac{\beta}{\bar{\theta}_{3}}\right)\right] \\
& \mathrm{C}_{0}=\frac{\bar{\theta}_{1}}{\beta} \frac{1}{\mathrm{k}_{11}^{2} \mathrm{k}_{44 \mathrm{v}}^{2}}\left[\mathrm{k}_{11}^{2}\left(\mathrm{k}_{44 \mathrm{v}}^{2}-\frac{\beta}{\bar{\theta}_{1}}\right)-\mathrm{k}_{44 \mathrm{v}}^{2}\left(\frac{\alpha_{1}^{2}}{\mathrm{C}_{11} \bar{\theta}_{1}}+\frac{\beta}{\bar{\theta}_{1}}\right)\right]
\end{aligned}
$$

where

$$
\begin{aligned}
& \bar{\theta}_{1}=s_{3} \theta_{1} \\
& \bar{\theta}_{3}=s_{1} \theta_{3} \\
& \bar{\alpha}_{1}=\alpha_{1} s_{3} \\
& \bar{\alpha}_{3}=\alpha_{3} s_{1}
\end{aligned}
$$




\section{Responses of Seismic Wave Propagation in Poroelastic Media Having Azimuthal Anisotropy}

The qualitative evaluation of the preferential directions of fluid flow in formations containing hydrocarbons is of great importance in the characterization of fractured reservoirs. Such preferential directions are related to the permeability anisotropy of the reservoir. Reservoirs are considered to be anisotropic when they possess significant variation in physical properties (porosity, permeability, wettability, etc.) in three dimensions. The permeability field at a given point in the rock can be treated as a second-order tensor in directions coincident with the principal permeability planes. In particular, the presence of vertical parallel cracks and fractures in an isotropic rock matrix leads to azimuthal anisotropy, which can be described by a transversely isotropic model with a horizontal axis of symmetry (Crampin, 1985). Azimuthal and incidence variations in P-wave attenuation and phase velocity from reverse VSP, cross-well seismic, and acoustic logging data have the potential to infer parameters associated with the fracture conditions of a reservoir. Recently, analytical studies to relate the tensor permeability to attenuation and dispersion of seismic waves has been conducted by Parra (1996). The results of Parra's work has lead to the development of an analytic solution that estimates the elements of the tensor permeability by modifiying the constitutive relation of the stress tensor in the pore fluid (Biot, 1955 and Biot, 1962). This constitutive equation was modified to describe the Biot and squirt-flow mechanisms for transversely isotropic poroelastic media. In addition, a field example was presented to test the model and to relate permeability with seismic waves propagating between wells at the Gypsy test site, Oklahoma (Collier, 1994; and Parra, et al., 1994). In this field example the horizontal permeability was controlled by cross-bedded and planarlaminated sandstones having an average permeability about three orders of magnitude greater than the average vertical permeability, which was associated with mudstones and siltstone bodies (permeability barriers). In this case the permeability anisotropy was described by a transversely isotropic model with vertical axis of symmetry.

In this paper, we present the theoretical solution of acoustic wave propagation in poroelastic media (including the Biot and squirt flow mechanisms based on the work of Dvorkin and Nur, 1993) to relate the directional dispersion and attenuation of $P$ and $S$-waves with azimuthal permeability anisotropy. In addition, numerical models are used to evaluate the sensitivity of attenuation and phase velocity to permeability anisotropy for several azimuthal variations and angles of incidence in the frequency range of cross-well seismic measurements and high-resolution reverse VSP (Owen and Parra, 1993, and Parra, 1995).

\section{Theory}

The formulation of the transversely isotropic poroelastic wave equation including the Biot and the squirt-flow mechanisms is based on the constitutive relations (i.e., the total stress tensor of the anisotropic porous medium and the stress tensor in the pore fluid), the momentum balance equation for total stress, and the generalized Darcy's law, in the framework of Biot's theory. These equations in the frequency-domain (assuming $\exp (-\mathrm{j} \omega \mathrm{t})$ variation) are (Kazi-Azoual, et al., 1988; Parra and Xu, 1994): 


$$
\begin{aligned}
\underset{\sim}{\sigma} & =\underset{c}{\mathbf{c}} \cdot \underset{\sim}{\mathbf{e}}-\underset{\sim}{\alpha} p, \\
\sigma_{\mathrm{ij}}^{(f)} & =-\phi p \delta_{\mathrm{ij}}=\frac{\phi}{\beta}[\underset{\sim}{\alpha} \cdot \underset{\sim}{\mathbf{e}}+\phi \nabla \cdot(\mathbf{U}-\mathbf{u})] \delta_{\mathrm{ij}}, \\
\frac{\partial \sigma_{i j}}{\partial x_{j}} & =\nabla \cdot \underset{\sim}{\sigma}=-\omega^{2}\left[\rho_{s}(1-\phi) \mathbf{u}+\phi \rho_{\mathrm{f}} \mathbf{U}\right], \\
\mathbf{w} & =\phi(\mathbf{U}-\mathbf{u})=-\underset{\sim}{\mathbf{K}}(\omega)\left(\omega^{2} \rho_{\mathrm{f}} \mathbf{u}-\nabla p\right) / j \omega .
\end{aligned}
$$

In these equations, $\underset{\sim}{\sigma}$ is the total stress tensor of the saturated porous medium; $\mathbf{e}$ is the strain tensor of the porous medium; $p$ is the fluid pressure; $\phi$ is the porosity; $\mathbf{u}$ and $U$ are the particle displacement of the solid and the fluid, respectively, $\rho_{\mathrm{s}}$ and $\rho_{\mathrm{f}}$ are the solid and fluid densities. In addition, $\underset{\mathbf{c}}{\mathbf{c}}$ is the solid-frame stiffness tensor containing five independent drained elastic coefficients (i.e., $c_{11}, c_{12}, c_{13}$, $\mathrm{c}_{44}$, and $\mathrm{c}_{33}$ ). The poroelastic coefficients of the effective stress of the second-rank tensor $\underset{\sim}{\alpha}$ (which are obtained at zero pore pressure) are given by

$$
\alpha_{1}=1-\left(c_{11}+c_{12}+c_{13}\right) / 3 K_{s} \text {, }
$$

and

$$
\alpha_{3}=1-\left(2 c_{13}+c_{33}\right) / 3 K_{s} \text {, }
$$

where $\mathrm{K}_{\mathrm{s}}$ is the bulk modulus of the grains. On the other hand, $\beta$ is the compressibility coefficient determined under undrained conditions, given by

$$
\beta=\phi / \mathrm{K}_{\mathrm{f}}+(1-\phi) / \mathrm{K}_{s}-\left[2\left(\mathrm{c}_{11}+\mathrm{c}_{12}+2 \mathrm{c}_{13}\right)+\mathrm{c}_{33}\right] / 9 \mathrm{~K}_{\mathrm{s}}^{2},
$$

where $\mathrm{K}_{\mathrm{f}}$ is the fluid bulk modulus. In the last equation (4), $w$ is the displacement of the fluid relative to solid, and $\mathbf{K}(\omega)$ is the frequency-dependent generalized Darcy's tensor described by two permeability constants. After Biot (1956), Biot (1962) and Schmitt (1989), the Darcy's elements, $\mathrm{K}_{\ell}(\omega)$, in terms of the complex permeability elements, $\kappa_{\ell}(\omega)$, for a low-frequency range are given by

$$
K_{l}(\omega)=\frac{K_{l}(\omega)}{\eta}=\frac{j \phi}{\omega \rho_{f}}\left[\frac{\rho_{\mathrm{a}} / \rho_{\mathrm{f}}+\phi}{\phi}+\frac{j \omega_{l}}{\omega}\right]^{-1}
$$

where $\frac{\omega \ell}{\omega}=\frac{\eta \phi}{\tilde{\mathrm{k}}_{\ell} \rho_{\mathrm{f}} \omega}$; for $\ell=1$ and 3 , and $\rho_{\mathrm{a}}$ is the additional density due to fluid (Biot, 1956). 
The parameter $\eta$ is the viscosity of the saturating fluid, and $\tilde{\mathrm{k}}_{l}$ is the intrinsic permeability along the principal direction $\ell$.

The total pressure for a 3D wave motion including the Biot and the squirt-flow mechanisms in a transversely isotropic medium in terms of the displacement of the fluid relative to the solid, $\mathbf{w}=\phi(\mathbf{U}-\mathbf{u})$, is given by (Parra, 1996)

$$
p=-\frac{1}{\beta}\left[s_{1} \nabla \cdot w+\left(s_{3}-s_{1}\right) \frac{\partial w_{x}}{\partial x}\right]-\frac{1}{\beta}\left[s_{1} \alpha_{3} \nabla \cdot u+\left(s_{3} \alpha_{1}-s_{1} \alpha_{3}\right) \frac{\partial u_{x}}{\partial x}\right] .
$$

where the elements of the squirt-flow tensor are given by

$$
\mathbf{s}_{\ell}=1-\frac{2 J_{1}\left(\gamma_{\ell} R_{l}\right)}{\gamma_{l} R_{l} J_{o}\left(\gamma_{l} R_{l}\right)}
$$

in which

$$
\begin{gathered}
\gamma_{\ell}^{2}=\frac{\rho_{\mathrm{f}} \omega^{2}}{(\phi / \beta)}\left[\frac{\rho_{\mathrm{a}} / \rho_{\mathrm{f}}+\phi}{\phi}+\frac{j \omega_{\ell}}{\omega}\right], \\
\frac{\omega_{\ell}}{\omega}=\frac{\eta \phi}{\tilde{\mathrm{k}}_{\ell} \rho_{\mathrm{f}} \omega} ; \text { for } \ell=1,3,
\end{gathered}
$$

and $R_{\ell}$ is the squirt-flow length for $\ell=1,3$.

For a transversely isotropic medium having the axis of anisotropy in the $x$-axis, the elements of the squirt tensor, $s_{3}$ and $s_{1}$ are oriented in the $x$ and $z$ directions, respectively.

\section{Plane Wave Solution of the Poroelastic Wave Equation}

The poroelastic system of differential equations associated with the vector wave displacement and the fluid pressure is obtained by eliminating the vector wave displacement of the fluid and the total stress tensor of the porous medium, using the constitutive equations (1), (3), and (4) and equation (8). This system of equations is reduced to one set of four coupled partial differential equations by assuming a poroelastic medium axially symmetric about the $\mathrm{x}$-axis: 


$$
\begin{array}{r}
{\left[c_{33} \frac{\partial^{2}}{\partial x^{2}}+c_{44}\left(\frac{\partial^{2}}{\partial y^{2}}+\frac{\partial^{2}}{\partial z^{2}}\right)+\omega^{2} \rho_{3}\right] u_{x}+\left(c_{13}+c_{44}\right)\left(\frac{\partial^{2} u_{z}}{\partial x \partial z}+\frac{\partial^{2} u_{y}}{\partial x \partial y}\right)-\alpha_{3} \frac{\partial p}{\partial x}=0} \\
\left(c_{13}+c_{44}\right) \frac{\partial^{2} u_{x}}{\partial x \partial y}+\left(c_{66}+c_{12}\right) \frac{\partial^{2} u_{z}}{\partial y \partial z}+\left[c_{44} \frac{\partial^{2}}{\partial x^{2}}+c_{11} \frac{\partial^{2}}{\partial y^{2}}+c_{66} \frac{\partial^{2}}{\partial z^{2}}+\omega^{2} \rho_{1}\right] u_{y}-\alpha_{1} \frac{\partial p}{\partial y}=0 \\
\left(c_{13}+c_{44}\right) \frac{\partial^{2} u_{x}}{\partial x \partial z}+\left(c_{12}+c_{66}\right) \frac{\partial^{2} u_{y}}{\partial y \partial z}+\left[c_{44} \frac{\partial^{2}}{\partial x^{2}}+c_{66} \frac{\partial^{2}}{\partial y^{2}}+c_{11} \frac{\partial^{2}}{\partial z^{2}}+\omega^{2} \rho_{1}\right] u_{z}-\alpha_{1} \frac{\partial p}{\partial z}=0 \\
-\alpha_{3} s_{1} \frac{\partial u_{x}}{\partial x}-\alpha_{1} s_{3} \frac{\partial u_{y}}{\partial y}-\alpha_{1} s_{3} \frac{\partial u_{z}}{\partial z}+\left[\theta_{3} s_{1} \frac{\partial^{2}}{\partial x^{2}}+\theta_{1} s_{3} \frac{\partial^{2}}{\partial y^{2}}+\theta_{1} s_{3} \frac{\partial^{2}}{\partial z^{2}}-\beta\right] p=0
\end{array}
$$

In order to solve the coupled system of equations (9), a plane harmonic wave in an arbitrary (x,y,z) plane is assumed to be represented by

$$
\left(u_{x}, u_{y}, u_{z}, p\right)=\left(U_{x}, U_{y}, U_{z}, P\right) \exp \left[j\left(\xi_{1} x+\xi_{2} y+\zeta z-c t\right)\right]
$$

This expression for a plane wave propagating in an anisotropic medium is substituted in equation (9) to yield

$$
\left[\begin{array}{cccc}
c_{33} \xi_{1}^{2}+c_{44}\left(\xi_{2}^{2}+\zeta^{2}\right)-\omega^{2} \overline{\rho_{3}} & \left(c_{13}+c_{44}\right) \xi_{1} \xi_{2} & \left(c_{13}+c_{44}\right) \xi_{1} \zeta & i \xi_{1} \overline{\alpha_{3}} \\
\left(c_{13}+c_{44}\right) \xi_{1} \xi_{2} & c_{44} \xi_{1}^{2}+c_{11} \xi_{2}^{2}+c_{66} \zeta^{2}-\omega^{2} \overline{\rho_{1}} & \left(c_{12}+c_{66}\right) \xi_{2} \zeta & i \xi_{2} \overline{\alpha_{1}} \\
\left(c_{13}+c_{44}\right) \xi_{1} \zeta & \left(c_{12}+c_{66}\right) \xi_{2} \zeta & c_{44} \xi_{1}^{2}+c_{66} \xi_{2}^{2}+c_{11} \zeta^{2}-\omega^{2} \overline{\rho_{1}} & \mathrm{i} \zeta \overline{\alpha_{1}} \\
\mathrm{i} \xi_{1} \overline{\alpha_{3}} & \mathrm{i} \xi_{2} \overline{\alpha_{1}} & \mathrm{i} \zeta \overline{\alpha_{1}} & \theta_{3} \xi_{1}^{2}+\theta_{1}\left(\xi_{2}^{2}+\zeta^{2}\right)+\beta
\end{array}\right]\left[\begin{array}{l}
\mathrm{U}_{\mathrm{x}} \\
\mathrm{U}_{\mathrm{y}} \\
\mathrm{U}_{z} \\
\mathrm{P}
\end{array}\right]=0
$$

The determinate of this system of equations (10) can be expressed by the product of two polynomials:

$$
\left(\mathrm{c}_{44} \xi_{1}^{2}+\mathrm{c}_{66} \Sigma^{2}-\omega^{2} \rho_{1}\right)\left[\mathrm{P}_{6}(\Sigma) \xi_{1}^{6}+\mathrm{P}_{4}(\Sigma) \xi_{1}^{4}+\mathrm{P}_{2}(\Sigma) \xi_{1}^{2}+\mathrm{P}_{\mathrm{o}}(\Sigma)\right]=0
$$

where 


$$
\begin{aligned}
\xi_{1}^{2} & =\frac{\omega^{2}}{\mathrm{c}^{2}} \sin ^{2} \Phi \cos ^{2} \theta \\
\xi_{2}^{2} & =\frac{\omega^{2}}{\mathrm{c}^{2}} \sin ^{2} \Phi \sin ^{2} \theta \\
\zeta^{2} & =\frac{\omega^{2}}{\mathrm{c}^{2}} \cos ^{2} \Phi \\
\text { and } \Sigma^{2} & =\zeta^{2}+\xi_{2}^{2}=\frac{\omega^{2}}{\mathrm{c}^{2}}\left(\cos ^{2} \Phi+\sin ^{2} \phi \sin ^{2} \theta\right)
\end{aligned}
$$

The first equation from (11) is given by

$$
\mathrm{C}_{44} \xi_{1}^{2}+\mathrm{C}_{66}\left(\xi_{2}^{2}+\zeta^{2}\right)-\omega^{2} \rho_{1}=0
$$

from which the propagation parameter associated with $\mathrm{qSH}$ is obtained,

$$
\xi_{\mathrm{qSH}}=\sqrt{\omega^{2} \rho_{1}-\frac{\left(c_{44} \xi_{1}^{2}+c_{66} \xi_{2}^{2}\right)}{c_{44}}}
$$

If we normalize the second dispersion equation by $\mathrm{P}_{6}(\Sigma)$, a third degree equation in $\xi_{1}^{2}$ can be written as follows:

$$
\xi_{1}^{6}+\mathrm{P}_{4} \xi_{1}^{4}+\mathrm{P}_{2} \xi_{1}^{2}+\mathrm{P}_{0}=0
$$

where

$$
\begin{gathered}
\mathrm{P}_{4}=\frac{\alpha_{3}^{2}}{\theta_{3} \mathrm{c}_{33}}+\mathrm{f}^{2}+\mathrm{v}^{2}+\chi^{2}+\mathrm{q}^{2} \\
\mathrm{P}_{2}=\alpha_{3}^{2}\left(\Sigma^{2} \epsilon^{2}-\frac{\omega^{2} \rho_{1}}{\mathrm{c}_{44}}\right) / \theta_{3} c_{33}+\mathrm{p}^{2}+\mathrm{f}^{2}\left(v^{2}+\chi^{2}+\mathrm{q}^{2}\right)
\end{gathered}
$$




$$
\text { and } P_{\circ}=\left(\frac{s_{3}}{s_{1}}\right) \alpha_{1}^{2} \Sigma^{2}\left(\Sigma^{2}-\omega^{2} \rho_{3} / c_{44}\right) / \theta_{3} c_{33}+f^{2} p^{2}
$$

in which

$$
\begin{aligned}
v^{2}=\Sigma^{2}-\omega^{2} \rho_{3} / c_{33} \\
\chi^{2}=\Sigma^{2}-\omega^{2} \rho_{1} / c_{44} \\
f^{2}=\left(\frac{s_{3}}{s_{1}}\right) \frac{\theta_{1}}{\theta_{3}} \Sigma^{2}+\beta / \theta_{3} s_{1} \\
\epsilon^{2}=c_{11} / c_{44}+c_{33}\left(\frac{s_{3}}{s_{1}}\right) \alpha_{1}^{2} / c_{44} \alpha_{3}^{2}-\alpha_{1}\left(1+\frac{s_{3}}{s_{1}}\right)\left(c_{13}+c_{44}\right) / \alpha_{3} c_{44} \\
q^{2}=\Sigma^{2}\left[c_{33}\left(c_{33}\left(c_{11}-2 c_{44}\right)-c_{13}\left(c_{13}+2 c_{44}\right)\right] / c_{33} c_{44}\right. \\
\text { and } p^{2}=\left(c_{11} / c_{33}\right)\left(\Sigma^{2}-\rho_{3} \omega^{2} / c_{44}\right)\left(\Sigma^{2}-\rho_{1} \omega^{2} / c_{11}\right)
\end{aligned}
$$

In order to determine the phase velocity and attenuation in terms of the angle of propagation $\Phi$ and the azimuthal angle $\theta$, the equations (12a) and (12b) are substituted in Equation (15), and Equation (12a) is substituted in the coefficients $P_{0}, P_{2}$ and $P_{4}$ given by Equations (16). The resulting dispersion equation is given by the third degree in $\mathrm{c}^{2}$ :

$$
c^{6}+c^{4} \omega^{2} h_{2}(\omega, \Phi, \theta)+c^{2} \omega^{4} h_{1}(\omega, \Phi, \theta)+\omega^{6} h_{o}(\omega, \Phi, \theta)=0
$$

where the coefficients $h_{o}(\Phi, \omega, \theta), h_{1}(\Phi, \omega, \theta)$ and $h_{2}(\Phi, \omega, \theta)$ are given in Appendix A.

Simple formulas can be deduced for a propagation angle of $\Phi=90^{\circ}$ and azimuth angle $\theta=0^{\circ}$, that is, when the motion is in the direction of the minimum permeability $\left(\tilde{\mathrm{k}}_{\mathrm{x}}\right)$ and the azimuthal axis is in the direction of the $x$-axis of anisotropy $\left(\theta=0^{\circ}\right)$. In this case the dispersion equation (17) is reduced to a biquadratic equation, which has a structure analogous to that of the isotropic case (Parra, 1991). The phase velocity of both qP waves (fast and slow) are solution of the bi-quadratic equation

$$
c^{6} k_{1}^{2} k_{2}^{2} k_{44 h}^{2}-\omega^{2} c^{4}\left\{k_{44 h}^{2}\left(k_{1}^{2}+k_{2}^{2}\right)+k_{1}^{2} k_{2}^{2}\right\}+c^{2} \omega^{4}\left(k_{1}^{2}+k_{2}^{2}+k_{44 h}^{2}\right)+\omega^{6}=0
$$

which can be reduced to 


$$
\left(k_{44 h}^{2} c^{2}-\omega^{2}\right)\left(k_{1}^{2} c^{2}-\omega^{2}\right)\left(k_{2}^{2} c^{2}-\omega^{2}\right)=0
$$

The solution of this equation is given by

$$
\mathrm{k}_{1,2}=\frac{1}{2}\left\{\left(\mathrm{k}_{\mathrm{p}}^{2}+\mathrm{k}_{\alpha}^{2}+\mathrm{k}_{\beta}^{2}\right) \pm \sqrt{\left(\mathrm{k}_{\mathrm{p}}^{2}+\mathrm{k}_{\alpha}^{2}+\mathrm{k}_{\beta}^{2}\right)^{2}-4 \mathrm{k}_{\mathrm{p}}^{2} \mathrm{k}_{\beta}^{2}}\right\},
$$

where

$$
\begin{aligned}
\mathrm{k}_{\mathrm{p}}^{2} & =\omega^{2} \rho_{3} / \mathrm{c}_{33} \\
\mathrm{k}_{\alpha}^{2} & =-\alpha_{3}^{2} / \theta_{3} c_{33} \\
\mathrm{k}_{\beta}^{2} & =-\beta / \theta_{3} \mathrm{~s}_{1} \\
\mathrm{k}_{44 \mathrm{~h}}^{2} & =\omega^{2} \rho_{1} / \mathrm{c}_{44} \\
\rho_{1} & =\rho+\rho_{\mathrm{f}}^{2} \omega^{2} \theta_{1} \\
\rho_{3} & =\rho+\rho_{\mathrm{f}}^{2} \omega^{2} \theta_{3} \\
\bar{\alpha}_{3} & =\alpha_{3}+\rho_{\mathrm{f}} \omega^{2} \theta_{3} \\
\rho & =(1-\phi) \rho_{s}+\phi \rho_{\mathrm{f}} \\
\text { and } \mathrm{s}_{1} & =1-2 \mathrm{~J}_{1}\left(\gamma_{1} \mathrm{R}_{1}\right) / \gamma_{1} \mathrm{R}_{1} \mathrm{~J}_{\mathrm{o}}\left(\gamma_{1} \mathrm{R}_{1}\right)
\end{aligned}
$$

In this case the $\mathrm{qP}$-wave velocity is controlled by the bulk modulus $\mathrm{c}_{33}$, and the attenuation is controlled by the squirt flow associated with the maximum permeability in the yz plane. In a similar manner, closed form expressions can be derived for a propagation angle of $\Phi=0^{\circ}$ and azimuth angle of $\theta=90^{\circ}$. In this case the wave motion is in the direction normal to the axis of anisotropy, and the azimuthal axis is parallel to the direction of maximum permeability $\left(\tilde{\mathrm{k}}_{\mathrm{z}}\right)$. The dispersion equation (17) is also reduced to a bi-quadratic equation similar to that given by Equation (18). This equation can be written as

$$
\left(k_{44 v}^{2} c^{2}-\omega^{2}\right)\left(k_{1}^{2} c^{2}-\omega^{2}\right)\left(k_{2}^{2} c^{2}-\omega^{2}\right)=0
$$


which has a solution of the same form as that of Equation (20). In this case, however, the wavenumbers are given by

$$
\begin{aligned}
\mathrm{k}_{\mathrm{p}}^{2} & =\omega^{2} \rho_{1} / c_{11} \\
\mathrm{k}_{\alpha}^{2} & =-\alpha_{1}^{2} / \theta_{1} c_{11} \\
\mathrm{k}_{\beta}^{2} & =-\beta / \theta_{1} s_{3} \\
\mathrm{k}_{44 \mathrm{v}}^{2} & =-\omega^{2} \rho_{3} / c_{11} \\
\rho_{1} & =\rho+\rho_{\mathrm{f}}^{2} \omega^{2} \theta_{1} \\
\alpha_{1} & =\alpha+\rho_{\mathrm{f}} \omega^{2} \theta_{1} \\
\rho & =(1-\phi) \rho_{\mathrm{s}}+\phi \rho_{\mathrm{f}} \\
\text { and } \mathrm{s}_{3} & =1-2 \mathrm{~J}_{1}\left(\gamma_{3} \mathrm{R}_{3}\right) / \gamma_{3} \mathrm{R}_{3} \mathrm{~J}_{\mathrm{o}}\left(\gamma_{3} \mathrm{R}_{3}\right) .
\end{aligned}
$$

The phase velocity for waves traveling parallel to the directional maximum permeability is controlled by the bulk modulus $c_{11}$, and the attenuation is controlled by the squirt-flow associated with the minimum permeability (horizontal permeability in the $\mathrm{x}$ direction).

\section{Numerical Results}

Attenuation and dispersion curves were produced for the model parameters given in Table I. The curves as a function of the azimuth angle $\theta$ (measured from the horizontal $\mathrm{x}$-axis as shown in Figure 1) and angle of propagation or incident angle (measured from the z-axis) were calculated (as illustrated in Figures 2-5) for a range of frequencies $500-2500 \mathrm{~Hz}$, in steps of $1000 \mathrm{~Hz}$. For angles of propagation $\Phi$ near vertical, as the azimuth angle varies between 0 to $90^{\circ}$, the attenuation and phase velocity change very little at each frequency. For example, for an angle of propagation of $15^{\circ}$ the phase velocity at $2500 \mathrm{~Hz}$ varies from $3117 \mathrm{~m} / \mathrm{s}$ at $\theta=0^{\circ}$ to $3149 \mathrm{~m} / \mathrm{s}$ at $\theta$ $=90^{\circ}$, i.e., about one percent. On the other hand, for an angle of propagation of $90^{\circ}$, the phase velocity at $2500 \mathrm{~Hz}$ varies from $2769 \mathrm{~m} / \mathrm{s}$ at $\theta=0^{\circ}$ to $3149 \mathrm{~m} / \mathrm{s}$ at $\theta=90^{\circ}$, i.e., about 14 percent and the attenuation increases more than 50 times at $\theta=90^{\circ}$. That is, the attenuation is minimum for angles of propagation in the direction parallel to the horizontal (minimum) permeability in the frequency range of $500-2500 \mathrm{~Hz}$. At any other angle of propagation the attenuation and phase velocity increases as the azimuth angle varies from 0 to $90^{\circ}$.

In the next example we analyze the effect of angle of propagation and azimuth on the attenuation and phase velocity for the selected frequency of $1500 \mathrm{~Hz}$ when the horizontal permeability is varied (see Figures 6-8). These figures show that as the incident angle $\Phi$ becomes perpendicular to the direction maximum permeability $\left(90^{\circ}\right)$, the attenuation is minimum [see the derivation of the special solution given by the bi-quadratic equation (18)]. In this case, the motion 
is controlled by the squirt-flow element, $s_{1}$, associated with the vertical permeability, $\tilde{\mathrm{k}}_{\mathrm{z}}=1000 \mathrm{md}$ as described by of Equations (20) and (21). For the vertical permeability of $1000 \mathrm{md}$, the attenuation peak corresponds to a frequency of about $100 \mathrm{kHz}$ and for the horizontal permeability, $\tilde{\mathrm{k}}_{\mathrm{x}}=2.5 \mathrm{md}$, the attenuation peak is at a frequency of about $1 \mathrm{kHz}$. Since the models that we are analyzing are in the range of $500-2500 \mathrm{~Hz}$, the attenuation associated with the vertical permeability of $1000 \mathrm{md}$ is negligible in this frequency range. This analysis suggests that it will require high frequency information to measure attenuation that is sensitive to the vertical permeability at the angle of propagation $\Phi=90^{\circ}$.

Even if the vertical permeability value can not be evaluated directly from the attenuation in the frequency range of either cross-well seismic measurements or acoustic logging, we can infer preferential directions of the fluid flow by analyzing dispersion and attenuations curves for different azimuth and incidence angles. For example, Figures $9 \mathrm{a}$ and $9 \mathrm{~b}$ show such curves produced in the frequency range of 50 to $10,000 \mathrm{~Hz}$ for the azimuth angles $0,30,60$, and 90 degrees and for incident angles of $45^{\circ}$ and $90^{\circ}$. The curves show that as the angle of incidence goes to $90^{\circ}$, there is a decrease on the attenuation for azimuthal angles less that $90^{\circ}$. In particular for $\Phi=90^{\circ}$ and $\theta$, $=0^{\circ}$ the attenuation is practically zero. Also the figures show that for $\theta=90^{\circ}$ the attenuation does not change when the angle of propagation varies from $45^{\circ}$ to $90^{\circ}$. In this case, the propagation is in the direction of maximum permeability $\left(\tilde{\mathbf{k}}_{\mathrm{z}}\right)$ coincident with the yz-plane

Alternatively, to predict the horizontal permeability, $\tilde{\mathrm{k}}_{\mathrm{x}}$, we need to understand the propagation characteristics at the angle of incidence, $\Phi=90^{\circ}$, i.e., when the azimuthal axis becomes in the plane parallel to the direction of maximum permeability or perpendicular to the axis of symmetry. This corresponds to maximum attenuation. In this case the attenuation is controlled by the horizontal squirt-flow element, $s_{3}$, which is associated with the horizontal permeability, $\tilde{\mathrm{k}}_{x}$. This suggests that processing techniques may be developed to extract the horizontal permeability from attenuation measurements at the azimuth of $90^{\circ}$ measured from the horizontal axis of symmetry.

Also, the horizontal permeability can be predicted for any azimuth when the angle of incidence approaches zero. In this case the effect of the azimuthal angle $\theta$ on the attenuation is negligible. Specifically, for $\Phi=0^{\circ}$ (normal incident) the attenuation and phase velocity is the same at all azimuthal angles. This can be proved analytical by making the angle $\Phi=0^{\circ}$ in the equations $A-$ 1, A-3, and A-5 in Appendix A. This condition reduces the functions $h_{0}, h_{1}$, and $h_{2}$ to the amplitude coefficients $A_{0}, B_{0}$, and $C_{0}$, respectively. These coefficients are given by equations $A-2, A-4$, and $A-6$ in the Appendix, and when they are replaced in equation (17) produce the same bi-quadratic equation given by equation (22). The form of this equation demonstrates that its solution [given by equation (20) with wavenumbers given by equation (23)] is independent of the azimuthal angle $\theta$.

The phase velocity curves shown in Figures $5 \mathrm{a}, 5 \mathrm{a}$, and $5 \mathrm{a}$ follow the same pattern as those of the attenuation. That is, as the azimuthal axis becomes parallel to the direction of maximum permeability, the phase velocity approaches the velocity of propagation parallel to the fracture plane, which corresponds to the maximum $\mathrm{qP}$-wave velocity associated with the parameter $\mathrm{c}_{11}$ [according to equation (22)]. On the other hand, as the azimuthal axis becomes parallel to the direction of horizontal permeability, the phase velocity approaches the velocity controlled by the stiffness constant $c_{33}$ [according to the equation (21)]. 
The curves shown in Figures 5-8 also show the effect of the horizontal permeability. The curves were calculated for horizontal permeability $\tilde{\mathrm{k}}_{\mathrm{h}}$ values of $2.5,7.5,12.5$, and $17.5 \mathrm{md}$. The attenuation decreases for horizontal permeabilities greater than $2.5 \mathrm{md}$. For example when the horizontal permeability increases from 2.5 to $7.5 \mathrm{md}$, the attenuation decreases about 30 percent for an angle of incidence of $15^{\circ}$ and azimuth $\theta=0^{\circ}$ and decreases about 90 percent for an angle of incidence of $90^{\circ}$. In this case the attenuation peak is shifted toward high frequencies; for a frequency of about $1500 \mathrm{~Hz}$, the attenuation values are in the low range of the attenuation curve.

TABLE I

Transversely isotropic poroelastic formation parameters.

\begin{tabular}{|c|c|c|}
\hline Parameter & Unit & Formation Values \\
\hline $\mathrm{K}_{\mathrm{s}}$ & $(\mathrm{GPa})$ & 37.9 \\
\hline$\rho_{s}$ & $\left(\mathrm{gr} / \mathrm{cm}^{3}\right)$ & 2.75 \\
\hline$v_{f}$ & $(\mathrm{~m} / \mathrm{s})$ & 1.5 \\
\hline$\rho_{\mathrm{f}}$ & $\left(\mathrm{gr} / \mathrm{cm}^{3}\right)$ & 1 \\
\hline$\eta$ & (poise) & 0.01 \\
\hline $\mathrm{c}_{11}$ & $(\mathrm{GPa})$ & 21 \\
\hline $\mathrm{c}_{13}$ & $(\mathrm{GPa})$ & 8.1 \\
\hline$c_{33}$ & $(\mathrm{GPa})$ & 18 \\
\hline $\mathrm{c}_{44}$ & $(\mathrm{GPa})$ & 5 \\
\hline$c_{66}$ & (GPa) & 6 \\
\hline$\phi$ & $\%$ & 23 \\
\hline$k_{h}$ & Darcy & 0.0025 \\
\hline $\mathrm{k}_{\mathrm{v}}$ & Darcy & 1 \\
\hline Sqh & $(\mathrm{mm})$ & 4 \\
\hline Sqv & $(\mathrm{mm})$ & 6 \\
\hline
\end{tabular}




\section{LIST OF CAPTIONS}

Figure 1. Three dimensional view of the anisotropic medium with its axis of symmetry perpendicular to the vertical z-axis, illustrating the orientation of the azimuthal angle $\theta$ and the angle of propagation $\Phi$ relative to Cartesian system of coordinates $(x, y, z)$.

Figure 2. The effect of azimuth angle and frequency for an angle of propagation of $\Phi=15^{\circ}$ in the range of frequencies $500-2500 \mathrm{~Hz}$. The horizontal permeability $k_{n}=2.5 \mathrm{md}$, and the vertical permeability $\mathrm{k}_{\mathrm{v}}=1000 \mathrm{md}$. (a) phase velocity, and (b) attenuation.

Figure 3. The effect of azimuth angle and frequency for an angle of propagation of $\Phi=30^{\circ}$ in the range of frequencies $500-2500 \mathrm{~Hz}$. The horizontal permeability $k_{b}=2.5 \mathrm{md}$, and the vertical permeability $\mathrm{k}_{\mathrm{v}}=1000 \mathrm{md}$. (a) phase velocity, and (b) attenuation.

Figure 4. The effect of azimuth angle and frequency for an angle of propagation of $\Phi=45^{\circ}$ in the range of frequencies $500-2500 \mathrm{~Hz}$. The horizontal permeability $k_{h}=2.5 \mathrm{md}$, and the vertical permeability $k_{v}=1000$ md. (a) phase velocity, and (b) attenuation.

Figure 5. The effect of azimuth angle and frequency for an angle of propagation of $\Phi=90^{\circ}$ in the range of frequencies $500-2500 \mathrm{~Hz}$. The horizontal permeability $k_{h}=2.5 \mathrm{md}$, and the vertical permeability $k_{v}=1000$ md. (a) phase velocity, and (b) attenuation.

Figure 6. The effect of azimuth angle and horizontal permeability for angle of propagation $\Phi=$ $15^{\circ}$ and a frequency of $1500 \mathrm{~Hz}$. (a) phase velocity, and (b) attenuation.

Figure 7. The effect of azimuth angle and horizontal permeability for angle of propagation $\Phi=$ $45^{\circ}$ and a frequency of $1500 \mathrm{~Hz}$. (a) phase velocity, and (b) attenuation.

Figure 8. The effect of azimuth angle and horizontal permeability for angle of propagation $\Phi=$ $90^{\circ}$ and a frequency of $1500 \mathrm{~Hz}$. (a) phase velocity, and (b) attenuation.

Figure 9. The effect of frequency and azimuth angles on the attenuation for incidence angles of (a) $45^{\circ}$, and (b) $90^{\circ}$. 


\section{REFERENCES}

Biot, M.A., 1955, Theory of elasticity and consolidation for porous anisotropic solid: J. Appl. Phys., 26, 182-185.

Biot, M.A., 1956, Theory of propagation of elastic waves in fluid-saturated porous rock: I. Lowfrequency range: J. Acoust. Soc. Am., 28, 167-178.

Biot, M.A., 1962, Mechanics of deformations and acoustic propagation in porous media: J. Appl. Phys., 33, 1482-1498.

Biot, M.A., 1962, Generalized theory of acoustic propagation i porous dissipative media: J. Acous. Soc. Am., 34, 1254-1264.

Collier, H.A., 1994, Using guided waves to characterize gas reservoir continuity Phase I: catalog of geological and petrophysical data from the Gypsy test site: Topical Report, Gas Research Institute, report no. GRI94/0384.4.

Crampin, S., 1985, Evidence for aligned cracks in the earth's crust: First Break, 3, 12-15.

Dvorkin, J. and Nur, A., 1993, Dynamic poroelasticity: a verified model with the squirt and the Biot mechanisms: geophysics, 58, 523-533.

Kazi-Azoual, M., 1988, Green's function in a infinite transversely isotropic saturated poroelastic medium: J. Acoust. Soc. Am., 84, 1883-1889.

Parra, J.O., 1991, Analysis of elastic wave propagation in stratified fluid-filled porous media for interwell seismic applications: Journal of the Acoustic Society of America, 90, 2557-2575.

Parra, J.O., Zook, B.J., and Sturdivant, V.R., 1994, Analysis and evaluation of interwell seismic logging techniques for hydrocarbon reservoir characterization: Final Report, Bartlesville Project Office, U.S. Dept. of Energy, Bartlesville, Oklahoma, Contract No. DE-AC2290BC14649.

Parra, J.O., and Xu, P.-C., 1994, Dispersive and attenuation of acoustic guided waves in layered fluid-filled porous media: J. Acoust. Soc. Am., 95, 91-98.

Parra, J.O., 1996, The transversely isotropic poroelastic wave equation including the Biot and the squirt mechanisms: theory and application: Geophysics, 62, 1-10.

Schmitt, 1989, D.P., 1988, Effects of radial layering when logging in saturated porous formations: J. Acoust. Soc. Am., 84, 220-2214. 


\section{APPENDIX A}

$$
\begin{aligned}
h_{0}(\omega, \Phi, \theta) & =A_{6} \sin ^{6} \Phi \cos ^{6} \theta+A_{4}\left(\sin ^{2} \Phi \sin ^{2} \theta+\cos ^{2} \Phi\right) \sin ^{4} \Phi \cos ^{4} \theta \\
& +A_{2} \sin ^{2} \Phi \cos ^{2} \theta\left(\sin ^{2} \Phi \sin ^{2} \theta+\cos ^{2} \Phi\right)^{2}+A_{0}\left(\sin ^{2} \Phi \sin ^{2} \theta+\cos ^{2} \Phi\right)^{3}
\end{aligned}
$$

where

$$
\begin{aligned}
\mathrm{A}_{6} & =\frac{\overline{\theta_{3}}}{\beta} \frac{1}{\mathrm{k}_{33}^{2} \mathrm{k}_{44 \mathrm{~h}}^{2}} \\
\mathrm{~A}_{4} & =\frac{\overline{\theta_{1}}}{\beta} \frac{1}{\mathrm{k}_{33}^{2} \mathrm{k}_{44 \mathrm{~h}}^{2}}+\frac{\overline{\theta_{3}}}{\beta} \mathrm{W}(\omega) \\
\mathrm{A}_{2} & =\frac{\overline{\theta_{3}}}{\beta} \frac{1}{\mathrm{k}_{11}^{2} \mathrm{k}_{44 \mathrm{v}}^{2}}+\frac{\overline{1}}{\beta} \mathrm{W}(\omega) \\
\mathrm{A}_{0} & =\frac{\overline{\theta_{1}}}{\beta} \frac{1}{\mathrm{k}_{11}^{2} \mathrm{k}_{44 \mathrm{v}}^{2}} \\
\mathrm{~W}(\omega) & =\frac{1}{\mathrm{k}_{33}^{2} \mathrm{k}_{11}^{2}}-\frac{1}{\mathrm{k}_{\mathrm{a}}^{2} \mathrm{k}_{\mathrm{b}}^{2}}+\frac{2}{\mathrm{k}_{44 \mathrm{~h}}^{2} \mathrm{k}_{\mathrm{b}}^{2}} \\
\mathrm{~h}_{1}(\omega, \phi, \theta)= & \mathrm{B}_{4} \sin ^{4} \Phi \cos ^{4} \theta+\mathrm{B}_{2} \sin ^{2} \Phi \cos ^{2} \theta\left(\sin ^{2} \Phi \sin ^{2} \theta+\cos ^{2} \Phi\right) \\
+ & \mathrm{B}_{0}\left(\sin ^{2} \Phi \sin ^{2} \theta+\cos ^{2} \Phi\right)^{2}
\end{aligned}
$$

where 


$$
\begin{aligned}
\mathrm{B}_{4} & =\frac{1}{\mathrm{k}_{33}^{2} \mathrm{k}_{44 \mathrm{~h}}^{2}}+\frac{1}{\beta} \frac{\bar{\alpha}_{3}^{2}}{\mathrm{c}_{33}} \frac{1}{\mathrm{k}_{33}^{2} \mathrm{k}_{44 \mathrm{~h}}^{2}}-\frac{\bar{\theta}_{3}}{\beta}\left(\frac{1}{\mathrm{k}_{44 \mathrm{~h}}^{2}}+\frac{1}{\mathrm{k}_{33}^{2}}\right) \\
\mathrm{B}_{2} & =\frac{1}{\beta} \frac{\overline{\alpha_{3}^{2}}}{\mathrm{c}_{33} \mathrm{k}_{11}^{2} \mathrm{k}_{33}^{2}}+\frac{1}{\beta} \frac{\overline{\alpha_{1}^{2}}}{\mathrm{c}_{11} \mathrm{k}_{11}^{2} \mathrm{k}_{33}^{2}}+\frac{1}{\beta \mathrm{k}_{11}^{2}}\left(\frac{1}{\mathrm{k}_{44 \mathrm{v}}^{2}}-\frac{1}{\mathrm{k}_{\mathrm{b}}^{2}}\right) \frac{\alpha_{1} \bar{\alpha}_{3}+\bar{\alpha}_{1} \alpha_{3}}{\mathrm{c}_{11}} \\
& -\frac{\bar{\theta}_{1}}{\beta}\left(\frac{1}{\mathrm{k}_{33}^{2}}+\frac{1}{\mathrm{k}_{44 \mathrm{~h}}^{2}}\right)-\frac{\bar{\theta}}{\beta}\left(\frac{1}{\mathrm{k}_{11}^{2}}+\frac{1}{\mathrm{k}_{44 \mathrm{v}}^{2}}\right)+\mathrm{W}(\omega) \\
\mathrm{B}_{0} & =\frac{1}{\beta} \frac{\overline{\alpha_{1}^{2}}}{\mathrm{c}_{11}} \frac{1}{\mathrm{k}_{11}^{2} \mathrm{k}_{44 \mathrm{v}}^{2}}-\frac{\bar{\theta}}{\beta}\left(\frac{1}{\mathrm{k}_{11}^{2}}+\frac{1}{\mathrm{k}_{44 \mathrm{v}}^{2}}\right)+\frac{1}{\mathrm{k}_{11}^{2} \mathrm{k}_{44 \mathrm{v}}^{2}} \\
\mathrm{~h}_{2}(\omega)= & \mathrm{C}_{2} \sin ^{2} \Phi \cos ^{2} \theta+\mathrm{C}_{0}\left(\sin ^{2} \Phi \sin ^{2} \theta+\cos ^{2} \Phi\right)
\end{aligned}
$$

where

$$
\begin{aligned}
& \mathrm{C}_{2}=\frac{\bar{\theta}_{3}}{\beta} \frac{1}{\mathrm{k}_{33}^{2} \mathrm{k}_{44 \mathrm{~h}}^{2}}\left[\mathrm{k}_{33}^{2}\left(\mathrm{k}_{44 \mathrm{~h}}^{2}-\frac{\beta}{\bar{\theta}_{3}}\right)-\mathrm{k}_{44 \mathrm{~h}}^{2}\left(\frac{\bar{\alpha}_{3}^{2}}{\mathrm{c}_{33} \theta_{3}}+\frac{\beta}{\bar{\theta}_{3}}\right)\right] \\
& \mathrm{C}_{0}=\frac{\bar{\theta}_{1}}{\beta} \frac{1}{\mathrm{k}_{11}^{2} \mathrm{k}_{44 \mathrm{v}}^{2}}\left[\mathrm{k}_{11}^{2}\left(\mathrm{k}_{44 \mathrm{v}}^{2}-\frac{\beta}{\bar{\theta}_{1}}\right)-\mathrm{k}_{44 \mathrm{v}}^{2}\left(-\frac{\alpha_{1}^{2}}{\mathrm{c}_{11} \bar{\theta}_{1}}+\frac{\beta}{\bar{\theta}_{1}}\right)\right]
\end{aligned}
$$

where

$$
\begin{aligned}
& \bar{\theta}_{1}=\mathrm{s}_{3} \theta_{1} \\
& \bar{\theta}_{3}=\mathrm{s}_{1} \theta_{3} \\
& \bar{\alpha}_{1}=\alpha_{1} s_{3} \\
& \bar{\alpha}_{3}=\alpha_{3} s_{1} \\
& k_{11}^{2}=\frac{\rho_{1} \omega^{2}}{c_{11}} ; k_{44 h}^{2}=\frac{\rho_{1} \omega^{2}}{c_{44}} \\
& k_{33}^{2}=\frac{\rho_{3} \omega^{2}}{c_{33}} ; k_{44 v}^{2}=\frac{\rho_{3} \omega^{2}}{c_{44}} \\
& k_{a}^{2}=\frac{\rho_{1} \omega^{2}}{c_{13}+2 c_{44}} ; k_{b}^{2}=\frac{\rho_{3} \omega^{2}}{c_{13}+2 c_{44}}
\end{aligned}
$$




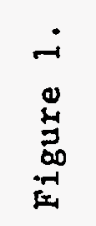

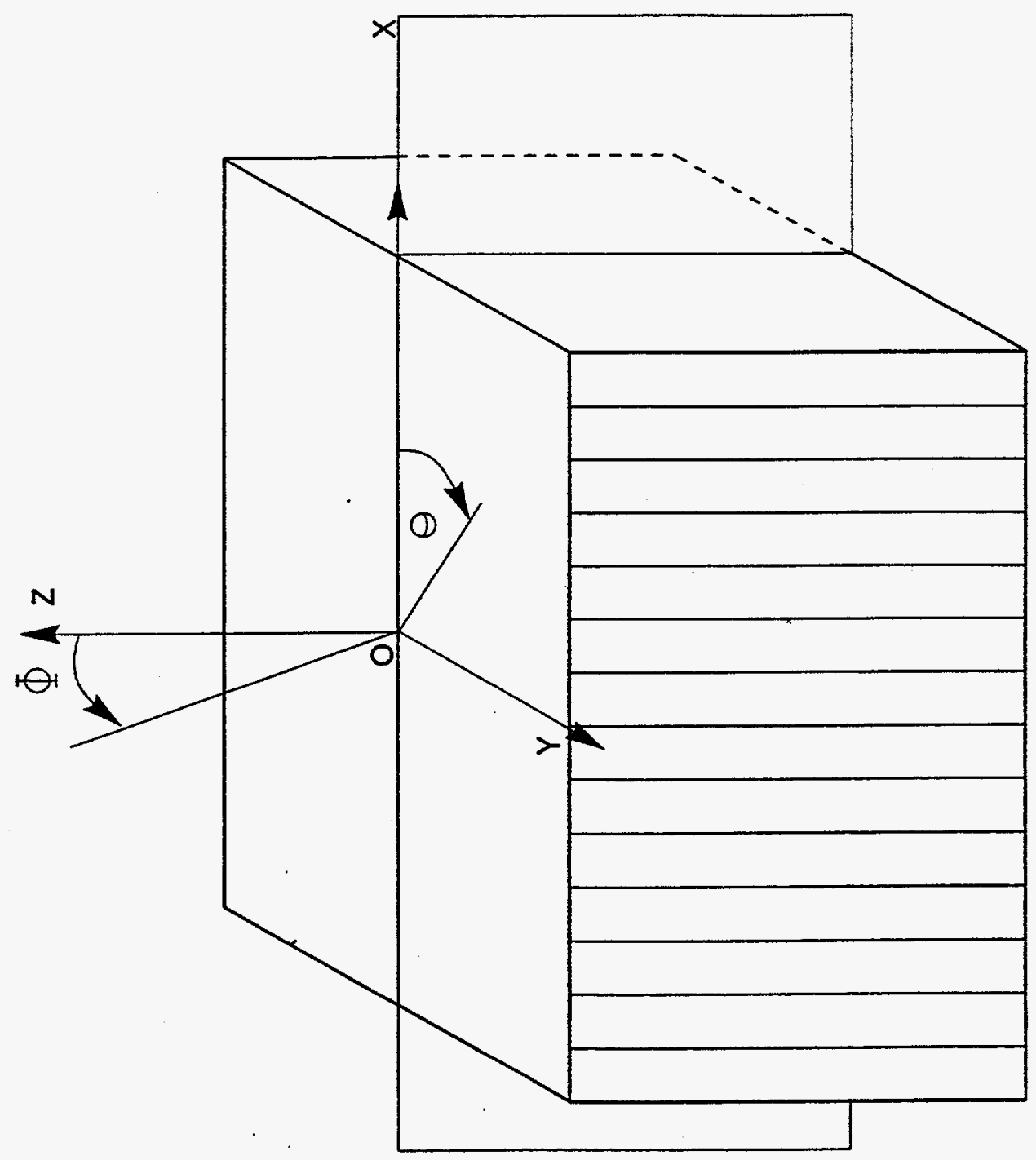




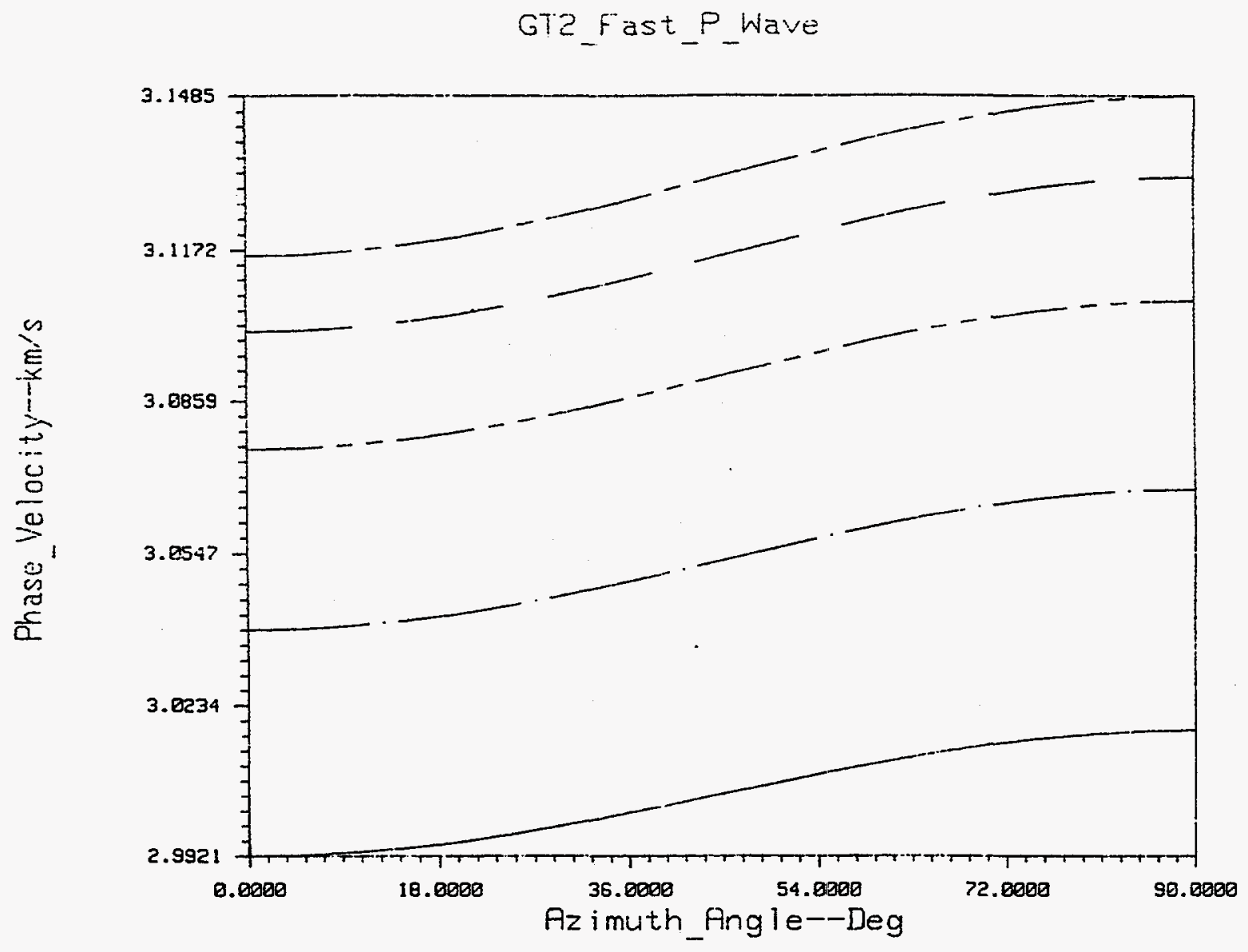

PHI $=15$ and $F=5801000150020002500$

$\mathrm{Kh}=$. BOeS and $\mathrm{KV}=1 \mathrm{D}$

Figure 2 a.

$$
\text { GT2_Fast_P_Wave }
$$

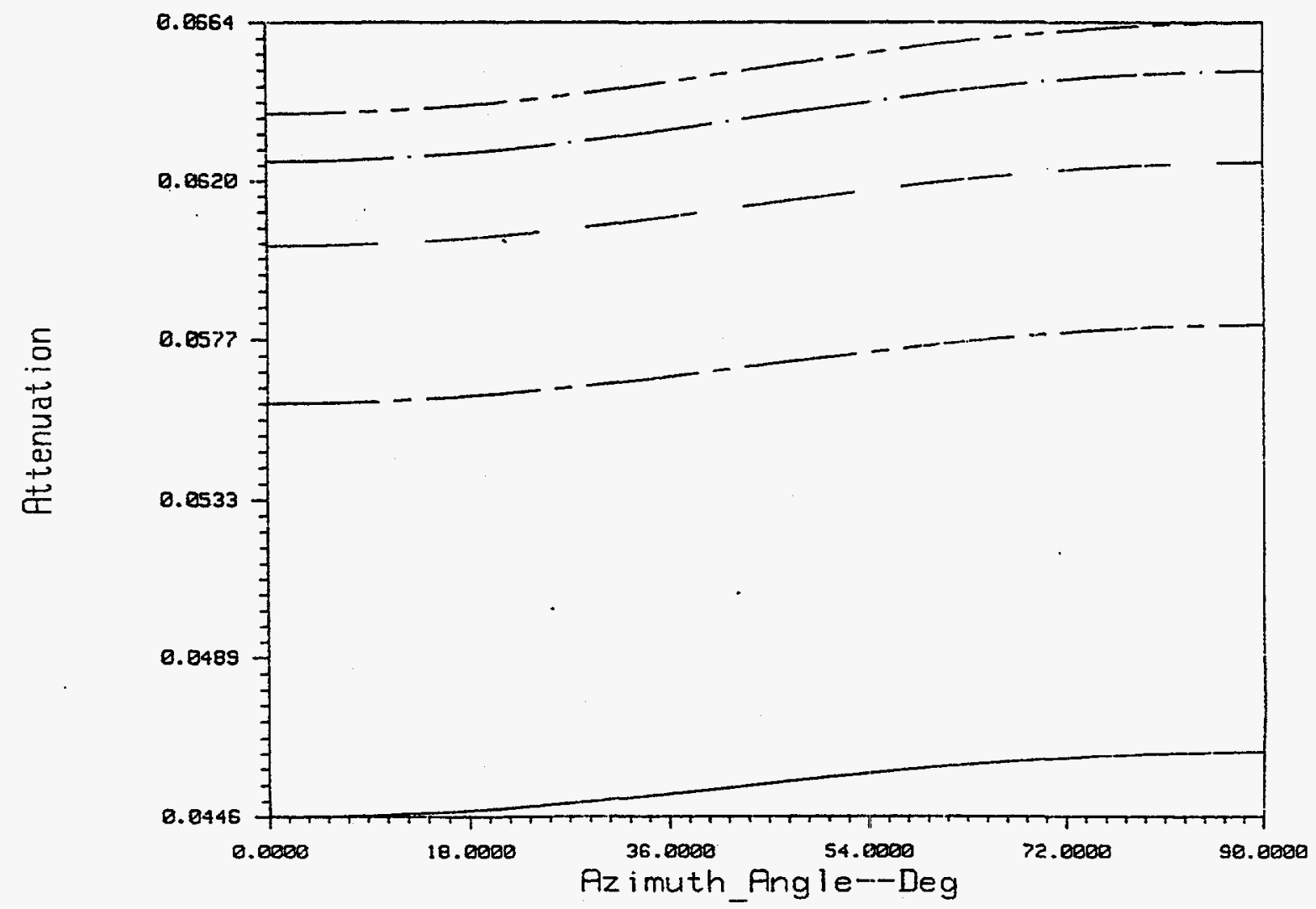




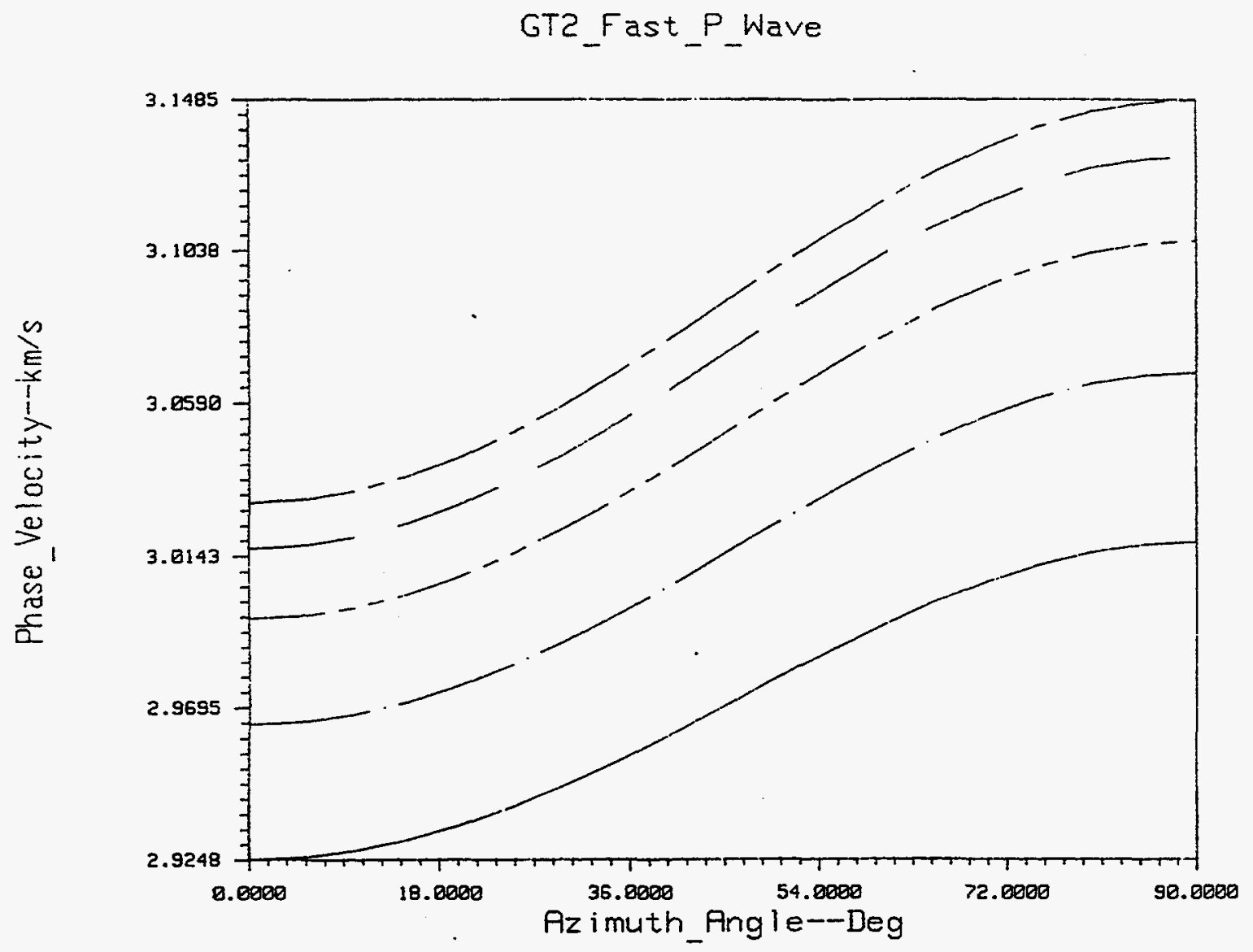

$P H I$ - 30 and $F$ - 5001800158028002500

$\mathrm{Kh}=.0025$ and $\mathrm{KV}_{\mathrm{V}}=1$. D

Figure 3a.

GT2_Fast_P_Wave

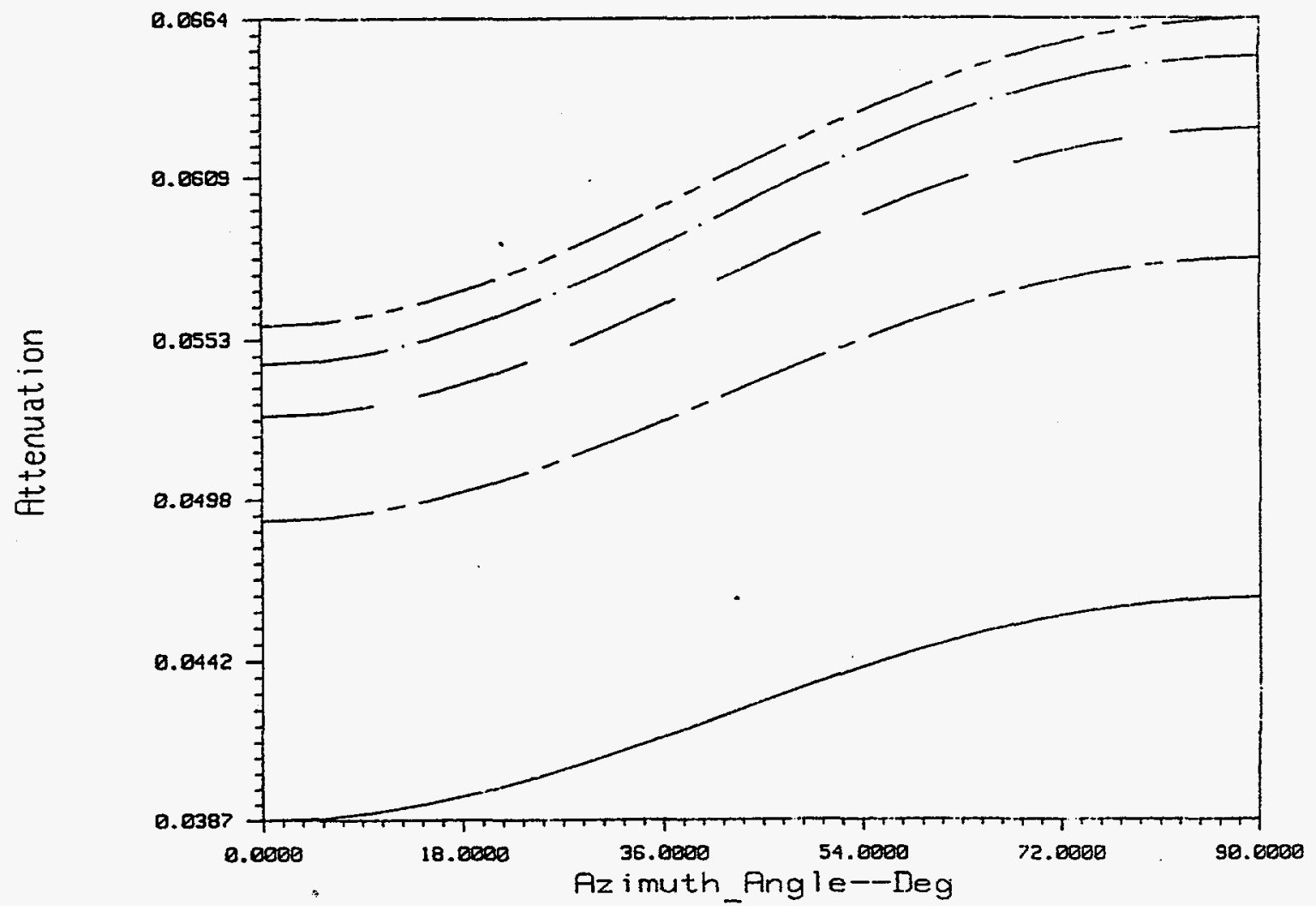

$\mathrm{PHI}=30$. and $F=5001000150020002500$

$k h=.8025$ and $K v=10$

Figure 3b. 
GT2_Fast_P_Wave

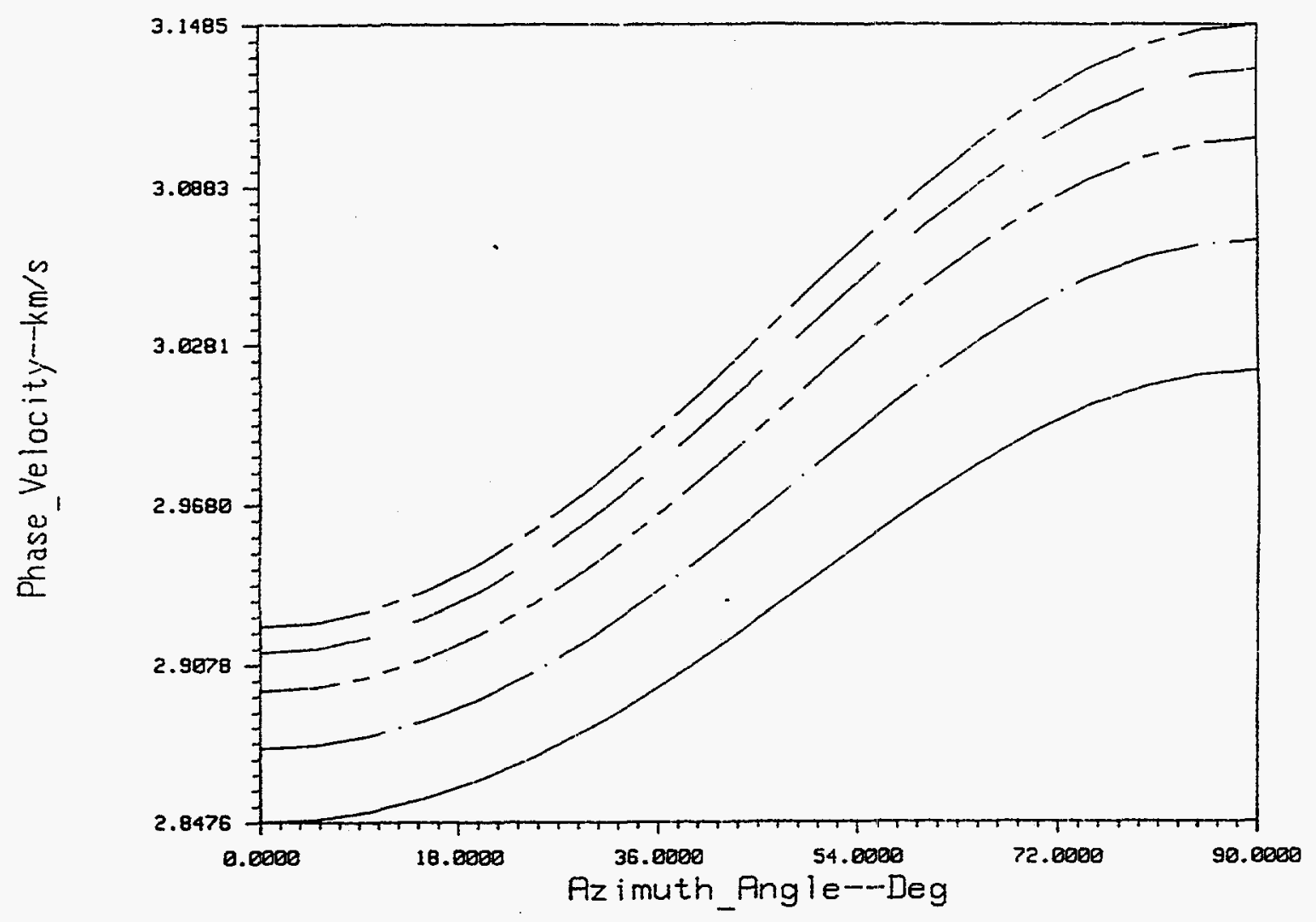

PHI $=45$ and $F=5601000150020002580$ $\mathrm{Kh}=.0025$ and $\mathrm{Kv}=1 \mathrm{D}$

Figure4a.

$$
\text { GT2_Fast_P_Wave }
$$

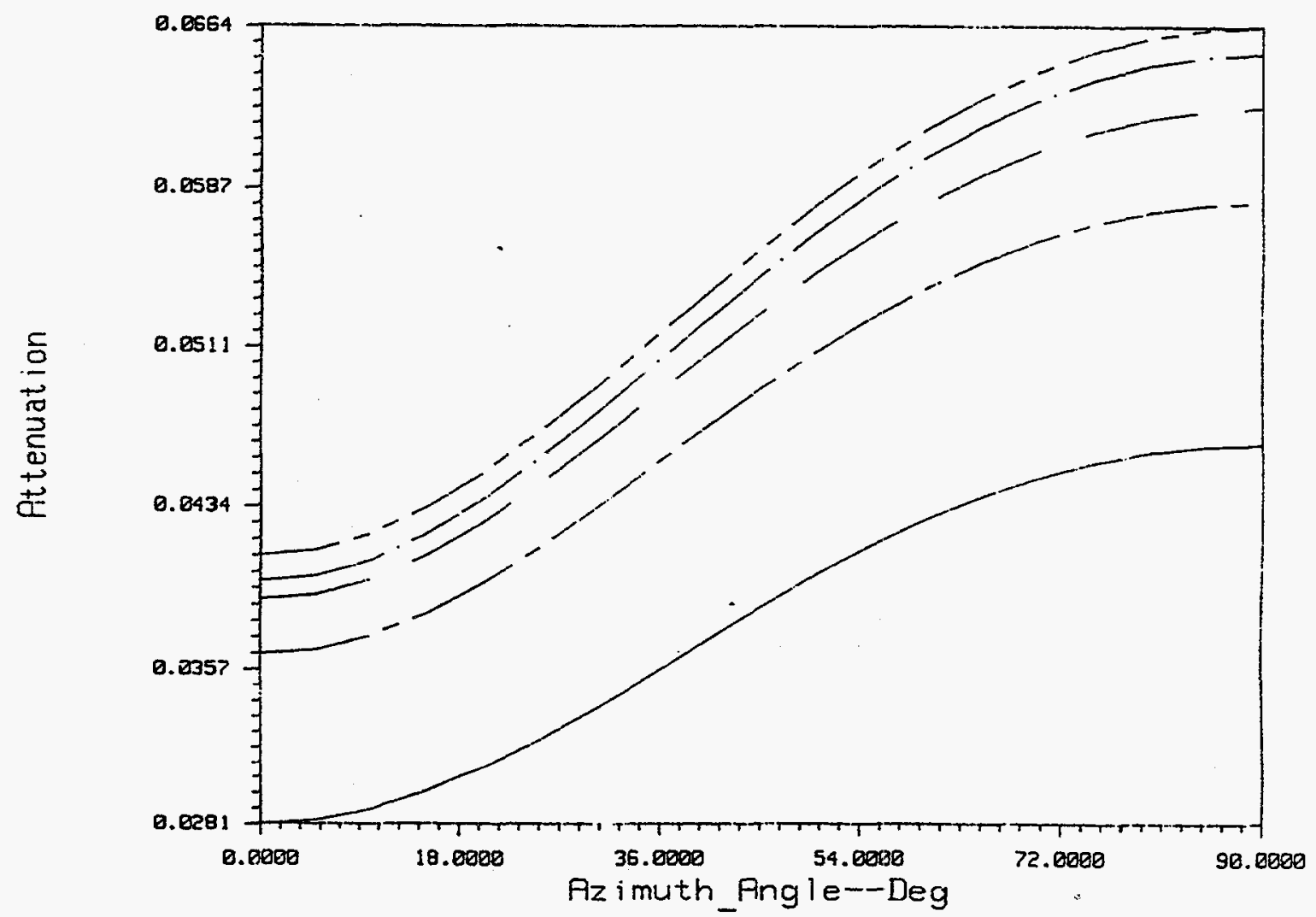


GT2_Fast_P_Wave

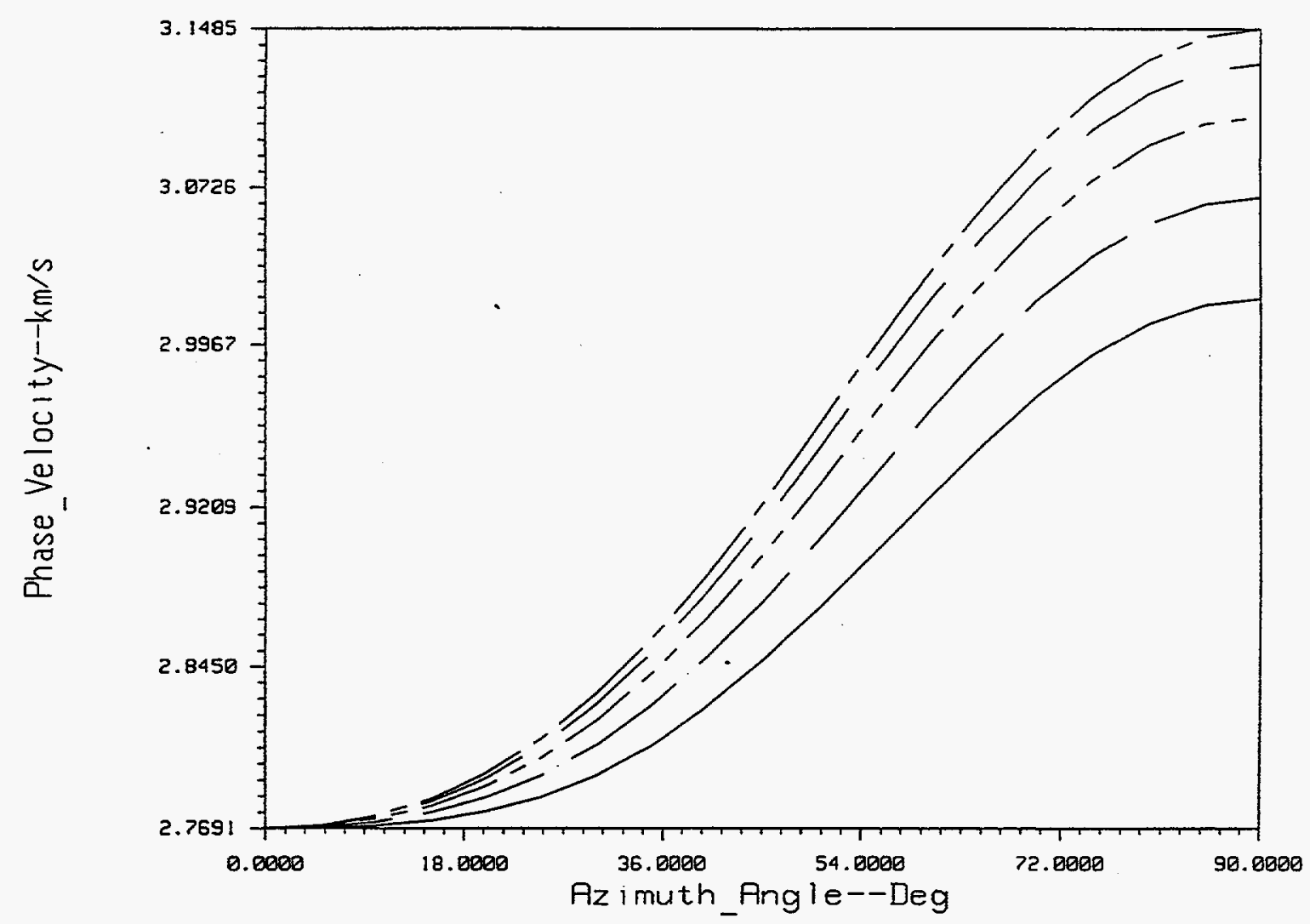

$\mathrm{PHI}=90$ and $F=5001000150020002500$ $k k=.8025$ and $k v=1$ Darcy

\section{GT2_Fast_P_Wave}

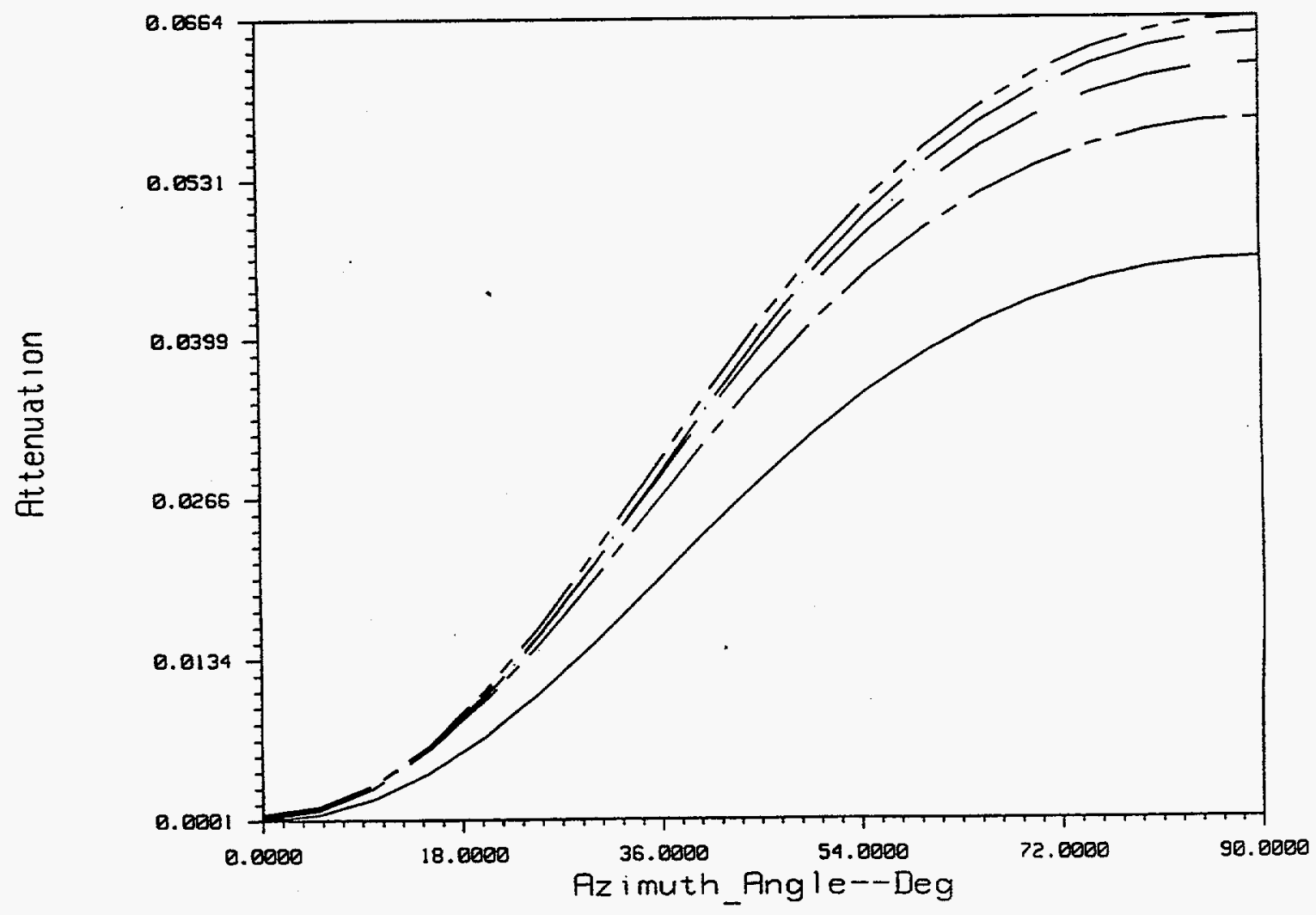

$P H I=90$. and $F=5801000158028002500$

$\mathrm{Kh}=.8025$ and $\mathrm{Kr}=1 \mathrm{D}$ 
TK2_Fast_P_Wave

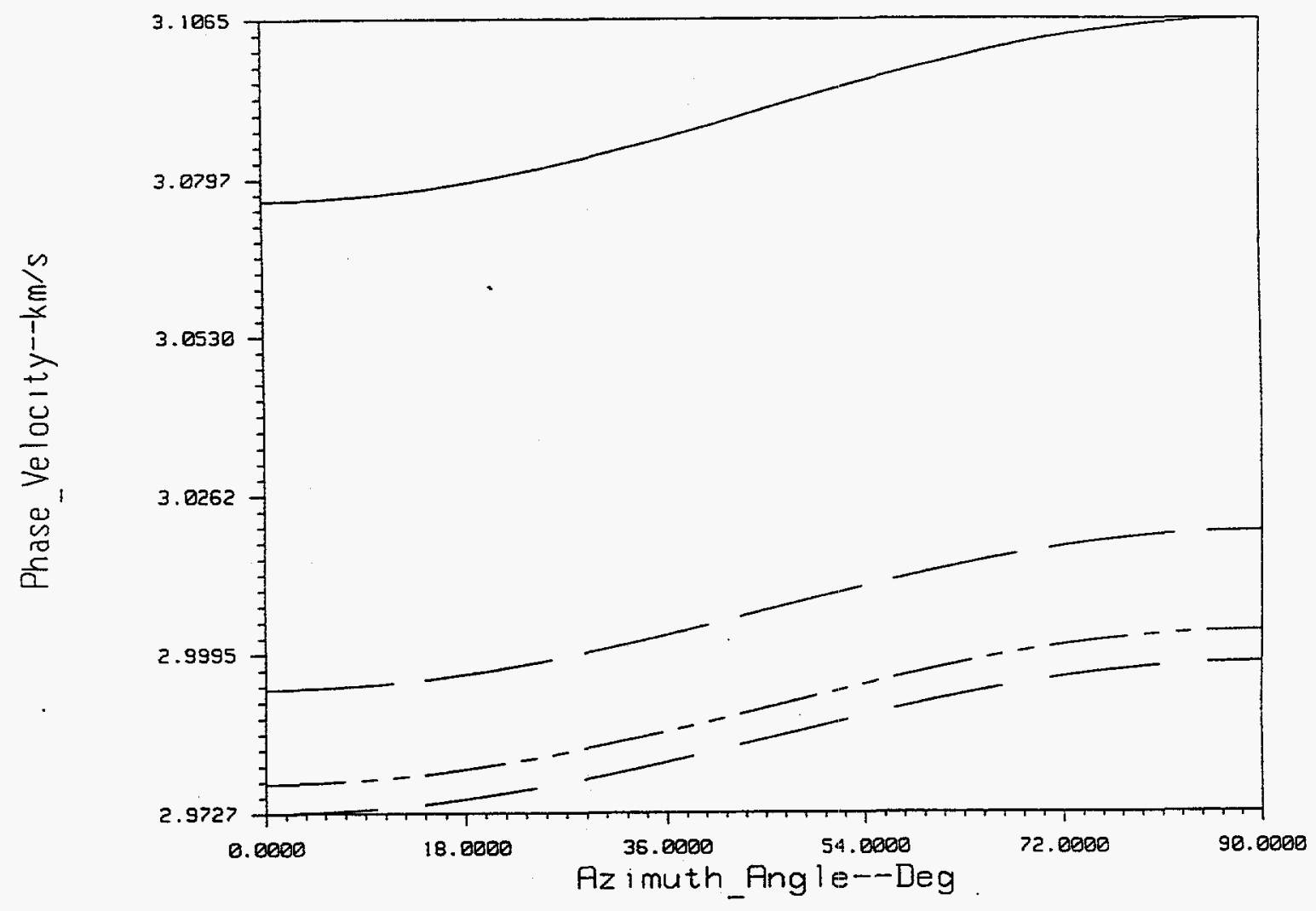

PHI $=15$ and $F=1500 \mathrm{~Hz}$

$k v=1$ and $k h=.0025 .005 .01 .02$

$$
\text { TK2_Fast_P_Wave }
$$

Figure 6a.

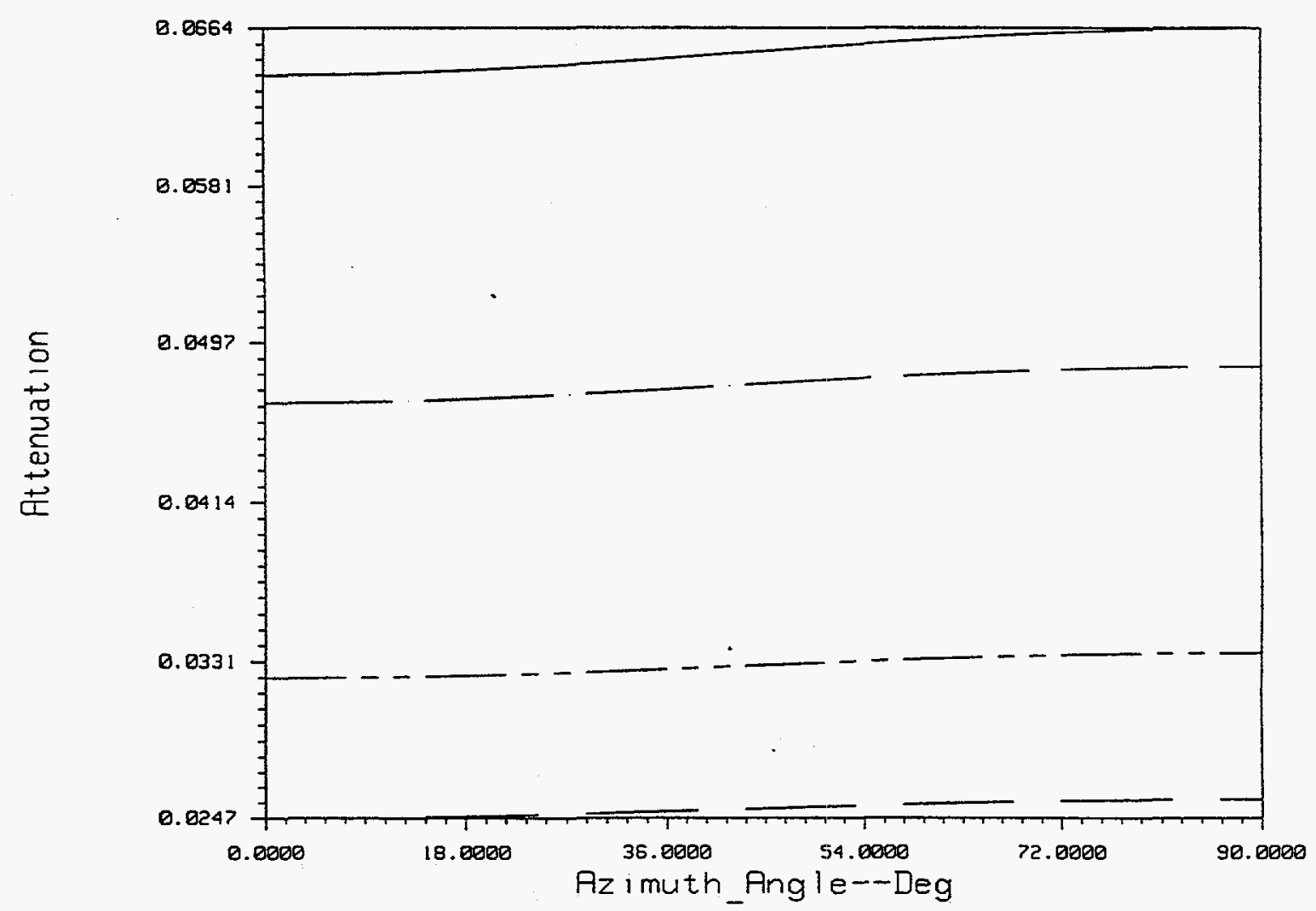


TK2_Fast_P_Wave

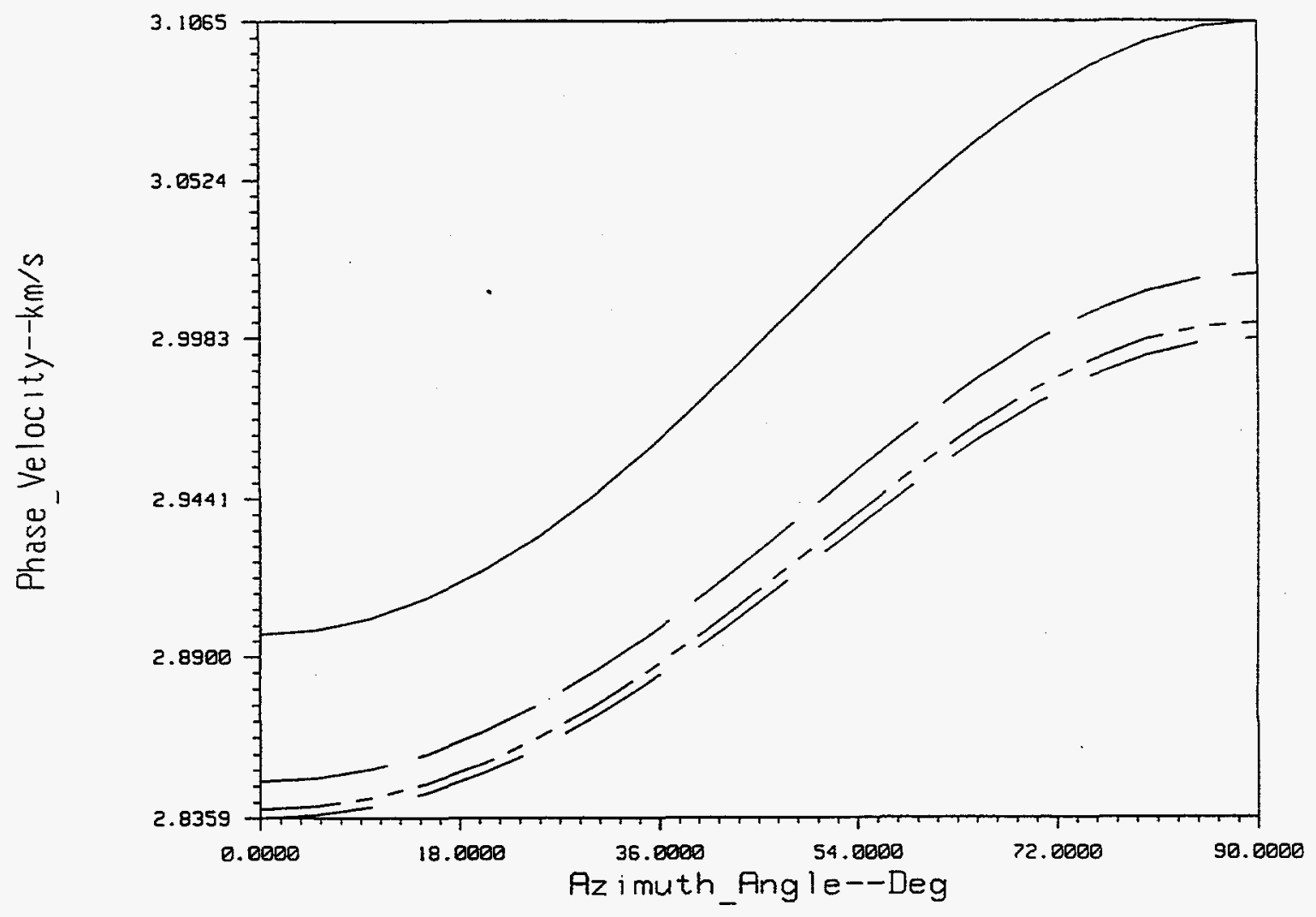

$\mathrm{PHI}=45$ and $F=1500 \mathrm{~Hz}$

$k v=1 \mathrm{D}$ and $k h=.0025 .005 .01 .02$

Figure $7 a$.

TK2 _Fast_P_Wave

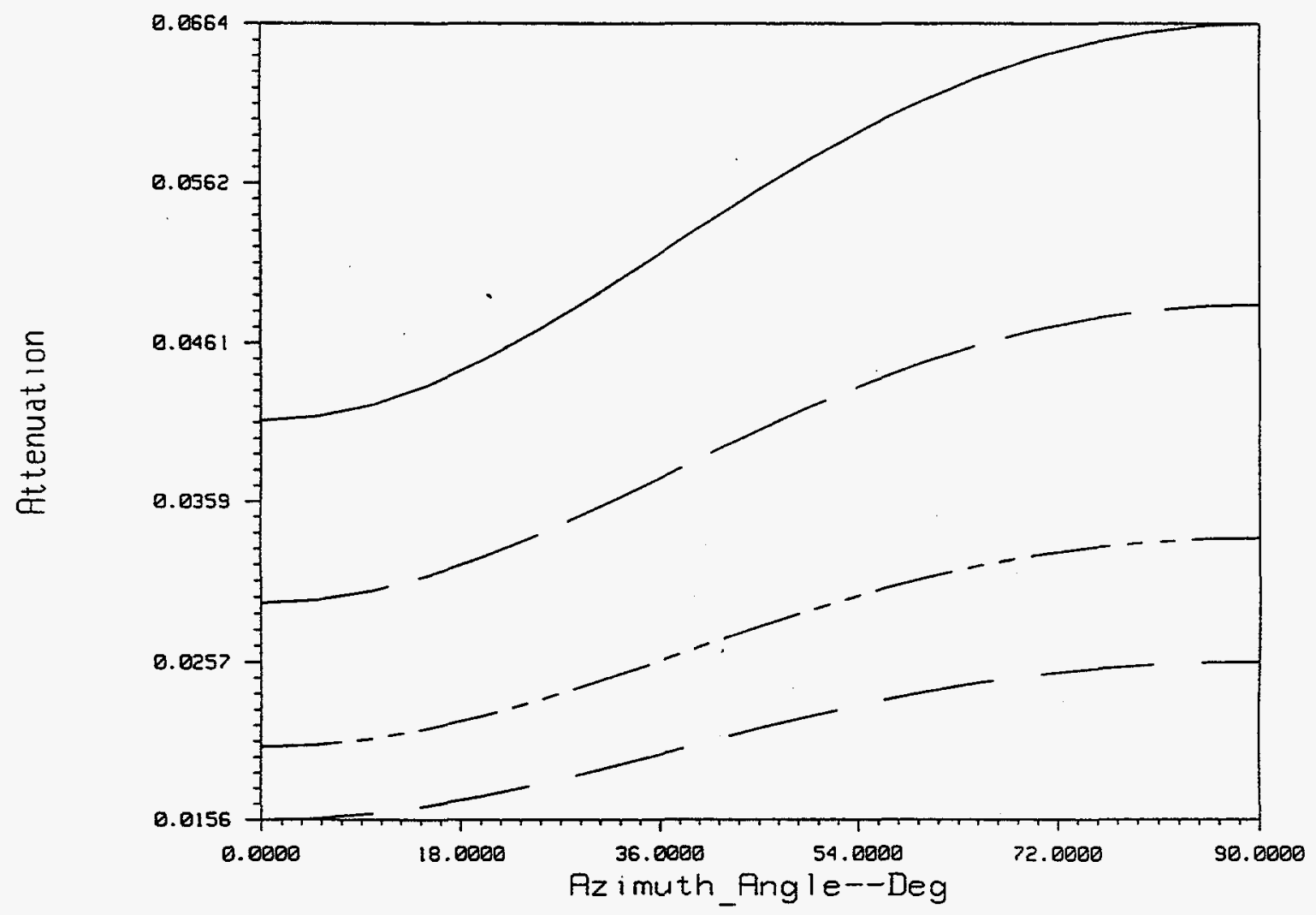

$P H I=45$ and $f=1580 \mathrm{~Hz}$

$k v=10$ and $k h=.0025 .005 .01 .02$ 
TK2 Fast_P_Wave

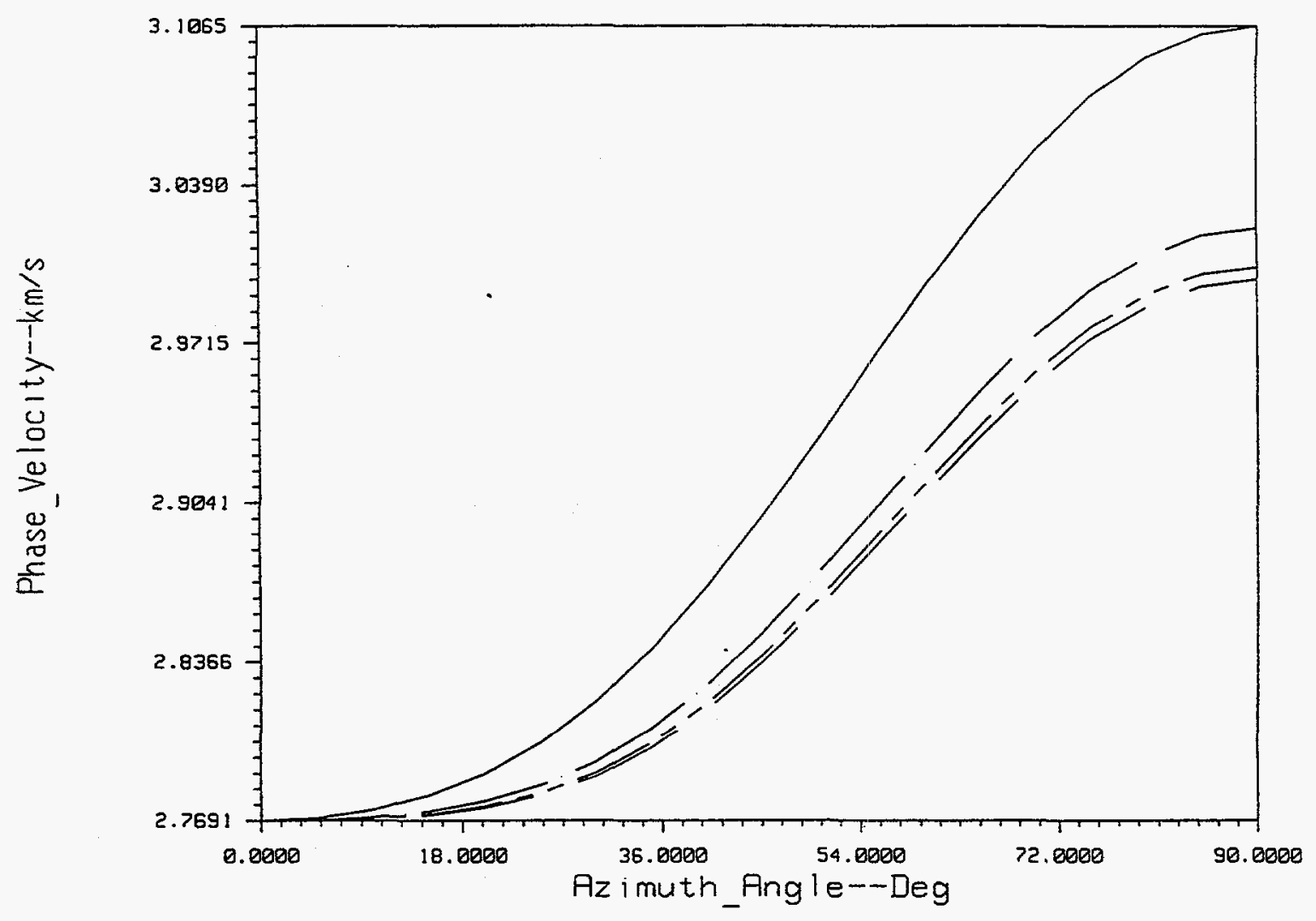

$\mathrm{PHI}=90$ and $\mathrm{F}=1500 \mathrm{~Hz}$

$k v=1 \mathrm{D}$ and $\mathrm{kh}=.0025 .005 .01 .02$

Figure $8 a$.

TK2_Fast_P_Wave

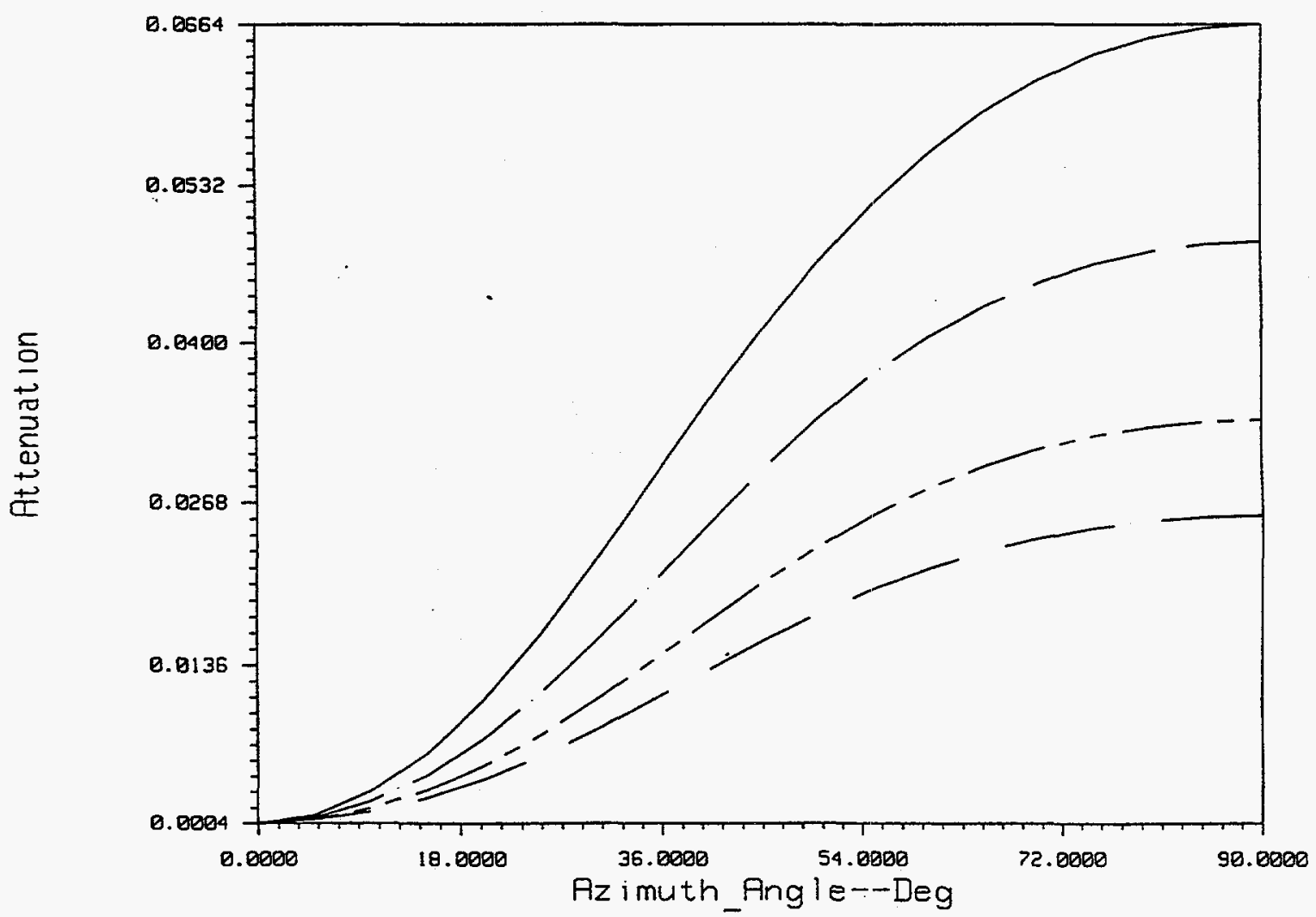

$P H I=90$ and $F=1500 \mathrm{~Hz}$

$k v=1$ and $k h=.0025 .005 .81 .82$ 


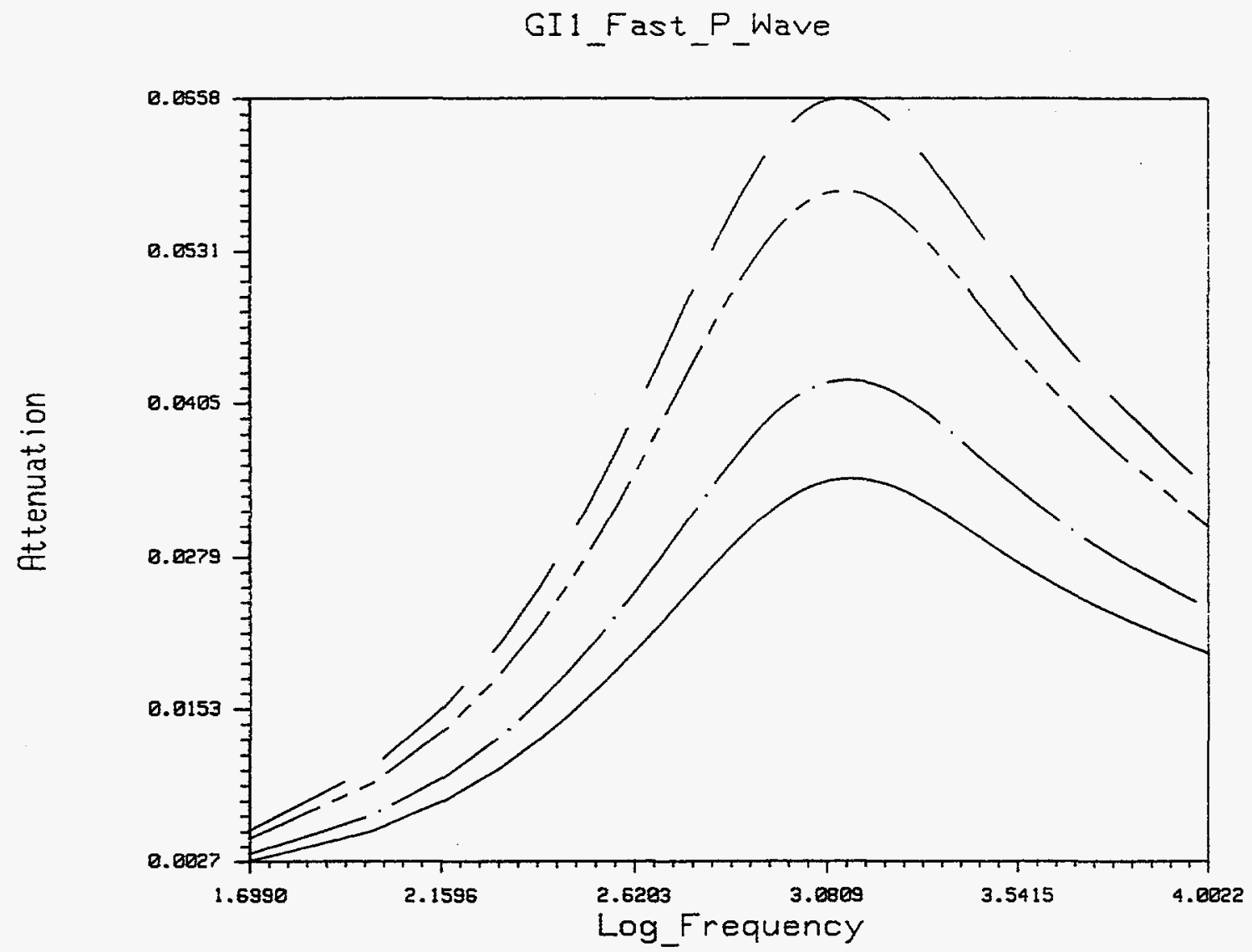

PHI $=45$ and THETANO 306898 $K h=.0025$ and $K_{V}=1$

Figure 9a.

GI1_Fast_P_Wave

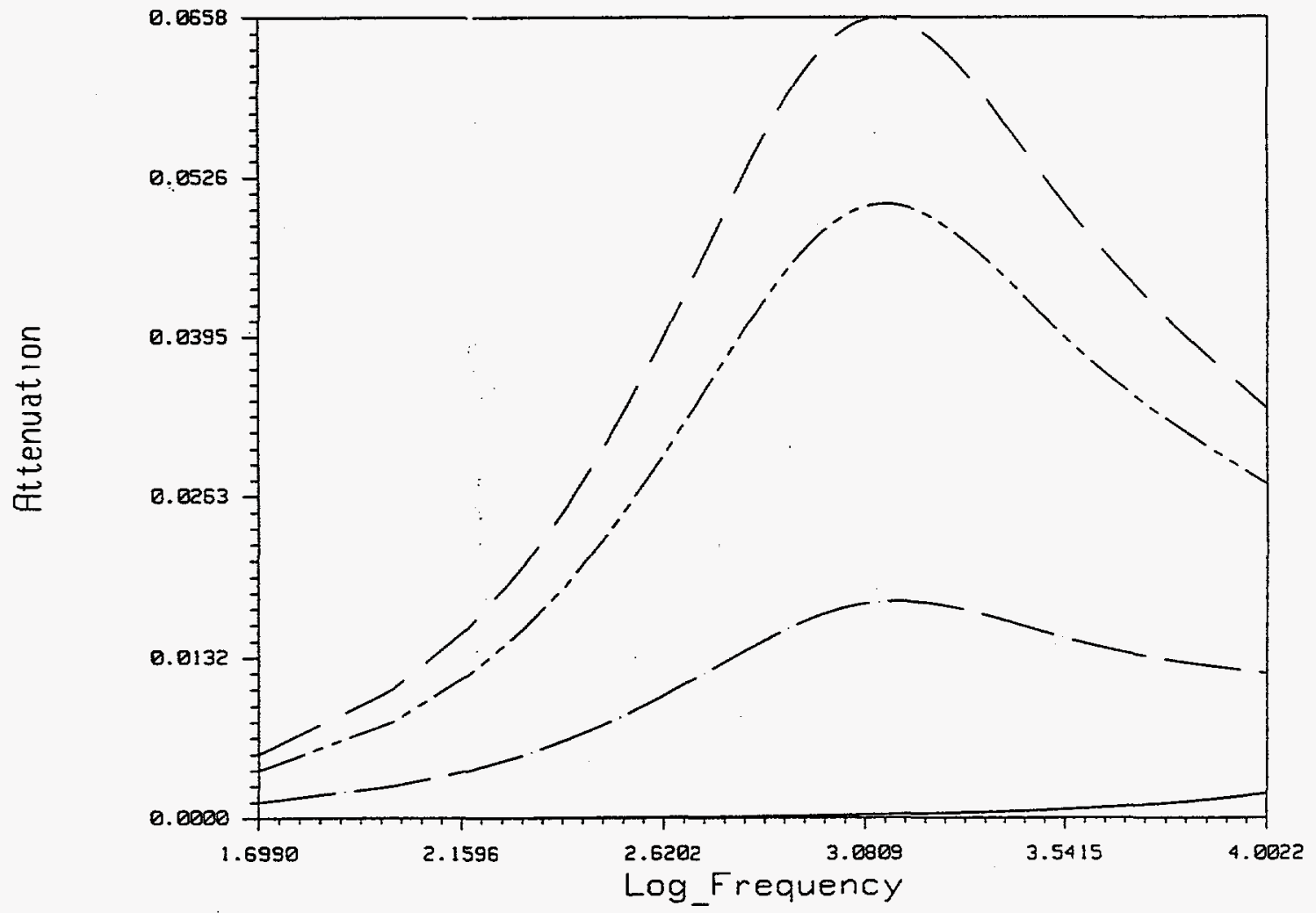

PHI $=90$ and Thet $a=0306090$

Figure $9 \mathrm{~b}$.

$K_{h}=.0025$ and $K_{v}=1$ Darcy 
III. ANALYSIS OF AN ACOUSTIC LOGGING SYSTEM IN A FLUID-FILLED BOREHOLE SOURROUNDED BY AN ANISOTROPIC POROELASTIC FORMATION

\section{A. The Response of an Acoustic Multipole in a Fluid-Filled Borehole Surrounded by a Poroelastic Formation}

This report presents a formulation for the acoustic wave due to a multipole in fluidfilled borehole surrounded by poroelastic formations. This approach obtains, in contrast to the approach of potentials ${ }^{[1]}$, a direct relationship between the displacement-stress-flux components of the formation and the coefficients of four types of waves, namely, fast quasi-P wave, slow quasi-P wave, SV wave, and SH wave, in terms of vertical wavenumber. As a result, the boundary conditions can be applied in a straightforward manner.

\section{Acoustic waves in the borehole}

Denote the radial, tangential, vertical displacements and pressure in the borehole fluid in cylindrical coordinates $(r, \vartheta, z)$ by $u_{r f}, u_{\vartheta \gamma}, u_{z f}$ and $p_{f}$, respectively. Using the Fourier transform with respect to time, $t$, we have

$$
\left\{\begin{array}{llll}
u_{r f} & u_{o f} & u_{z f} & p_{f}
\end{array}\right\}^{T}=\frac{1}{2 \pi} \int_{-\infty}^{\infty}\left\{\begin{array}{llll}
\left\{U_{r f}\right. & U_{o f} & U_{z f} & P_{f}
\end{array}\right\}^{T} e^{-i \omega t} d \omega
$$

Further, it can be shown by using integral transform with respect to $z$ that ${ }^{[2]}$

$$
\left\{\begin{array}{l}
U_{r f}(r, \mathfrak{v}, z) \\
U_{v f}(r, \tilde{v}, z) \\
U_{f f}(r, \tilde{v}, z) \\
P_{f}(r, \tilde{v}, z)
\end{array}\right\}=\int_{-\infty}^{\infty}\left\{\begin{array}{c}
\frac{\partial}{\partial r} \\
\frac{\partial}{r} \\
\frac{\partial}{\partial v} \\
\frac{\partial}{\partial z} \\
\omega^{2} \rho_{f}
\end{array}\right\}\left[\Phi_{f}^{0}(r, \tilde{v}, z, k)+\Phi_{\mathfrak{f}}^{R}(r, \tilde{v}, z, k)\right] d k
$$

where the frequency dependence has been suppressed. Superscript 0 represents the wave due to the source in an unbounded medium, $R$ represents the wave that is reflected from the borehole interface, and

$$
\Phi_{\mathrm{f}}^{0}(\mathrm{r}, \mathrm{U}, \mathrm{z}, \mathrm{k})=-\frac{\mathrm{i}}{4 \mathrm{n} !}\left(\frac{\xi_{\mathrm{f}} \mathrm{r} 0}{2}\right)^{\mathrm{n}} \epsilon_{\mathrm{n}} \mathrm{H}_{\mathrm{n}}\left(\xi_{\mathrm{f}} \mathrm{r}\right) \cos [\mathrm{n}(\boldsymbol{\mho}-\gamma)] \mathrm{e}^{\mathrm{ikz}}
$$




$$
\Phi_{f}^{R}(r, \boldsymbol{\mho}, z, k)=-\frac{i}{4 n !}\left(\frac{\xi_{f} r_{0}}{2}\right)^{n} \overline{A_{n}}(k) J_{n}\left(\xi_{f} r\right) \cos [n(\hat{\mho}-\gamma)] e^{i k z}
$$

where $n$ is the order of the multipole, $r_{0}$ and $\gamma$ its radius and azimuth angle, and $\epsilon_{0}=1 / 2 ; \epsilon_{n}=1, n$ $>0$. The vertical and radial wavenumbers, denoted by $k$ and $\xi_{f}$ are related through

$$
\begin{aligned}
\xi_{\mathrm{f}} & =\sqrt{\mathrm{k}_{\mathrm{f}}^{2}-\mathrm{k}^{2}} & \mathrm{k}_{\mathrm{f}} \geq \mathrm{k} \\
& =\mathrm{i} \sqrt{\mathrm{k}^{2}-\mathrm{k}_{\mathrm{f}}^{2}} & \mathrm{k}_{\mathrm{f}}<\mathrm{k} \\
\mathrm{k}_{\mathrm{f}} & =\frac{\omega}{V_{f}} &
\end{aligned}
$$

$V_{f}$ is the velocity of the acoustic wave in the fluid, and $\omega$ the circular frequency. $A_{n}$ in equation (4) is $k$-dependent and is determined from boundary conditions.

\section{Poroelastic waves in the formation}

Similarly to the acoustic waves in borehole fluid, denote the radial, tangential, vertical displacements and pressure in the formation and their Fourier transformed counterparts by $\left\{u_{r}, u_{\diamond}\right.$ $\left.u_{z}, p\right\}^{\mathrm{T}}$ and $\left\{U_{r}, U_{o}, U_{z}, P\right\}^{\mathrm{T}}$, respectively. The fast quasi-P wave, slow quasi-P wave, $\mathrm{SV}$-wave and $\mathrm{SH}$-wave in the formation can then be represented by their wave functions in the wavenumber domain $^{[1]}$ :

$$
\begin{aligned}
& \Phi_{1}(\mathrm{r}, \hat{\mathrm{v}}, \mathbf{z}, \mathrm{k})=\overline{\mathrm{B}}_{\mathrm{n}}(\mathrm{k}) \mathrm{H}_{\mathrm{n}}\left(\xi_{1} \mathrm{r}\right) \frac{1}{\mathrm{n} !}\left(\frac{\xi_{\mathrm{f}} \mathrm{r}_{0}}{2}\right)^{\mathrm{n}} \cos [\mathrm{n}(\mathfrak{\mho}-\gamma)] \mathrm{e}^{\mathrm{ikz}} \\
& \Phi_{2}(r, \hat{v}, z, k)=\bar{C}_{n}(k) H_{n}\left(\xi_{2} r\right) \frac{1}{n !}\left(\frac{\xi_{f} r_{0}}{2}\right)^{n} \cos [n(\hat{v}-\gamma)] e^{i k z} \\
& \Gamma(r, \delta, z, k)=\bar{D}_{n}(k) H_{n}\left(\xi_{3} r\right) \frac{1}{n !}\left(\frac{\xi_{f} r_{0}}{2}\right)^{n} \cos [n(\vartheta-\gamma)] e^{i k z} \\
& \Psi(r, \hat{v}, z, k)=\bar{E}_{n}(k) H_{n}\left(\xi_{4} r\right) \frac{1}{n !}\left(\frac{\xi_{f} r_{0}}{2}\right)^{n} \sin [n(\vartheta-\gamma)] e^{i k z}
\end{aligned}
$$

where $\xi_{j} j=1,2,3,4$ are radial wavenumbers corresponding to fast quasi-P wave, slow quasi-P wave, $\mathrm{SV}$ wave, and SH wave, respectively. These wavenumbers are given by ${ }^{[3]}$ 


$$
\begin{array}{rlr}
\xi_{\mathrm{j}} & =\sqrt{\lambda_{\mathrm{j}}^{2}-\mathrm{k}^{2}} \quad \lambda_{\mathrm{j}} \geq \mathrm{k} \\
& =\mathrm{i} \sqrt{\mathrm{k}^{2}-\lambda_{\mathrm{j}}^{2}} \quad \lambda_{\mathrm{j}}<\mathrm{k} \\
\lambda_{\mathrm{l}}^{2}+\lambda_{2}^{2} & =\mathrm{k}_{\mathrm{p}}^{2}-\frac{\frac{\alpha^{2}}{\theta(\lambda+2 \mu)}-\frac{\beta}{\theta}}{\lambda_{1}^{2} \lambda_{2}^{2}}=-\mathrm{k}_{\mathrm{p}}^{2} \frac{\beta}{\theta} \\
\lambda_{3}^{2} & =\lambda_{4}^{2}=\mathrm{k}_{\mathrm{s}}^{2} \\
\mathrm{k}_{\mathrm{p}}^{2} & =\frac{\omega^{2} \rho}{\lambda+2 \mu} \\
\mathrm{k}_{\mathrm{s}}^{2} & =\frac{\omega^{2} \rho}{\mu}
\end{array}
$$

where $\rho$ is the mass density, and $\alpha, \beta$ and $\theta$ are constants associated with the porosity and permeability. The overbar symbol indicates a parameter modified by the porosity and permeability. Since these components are linearly related to the wave functions in $(6)^{[1]}$, we can write

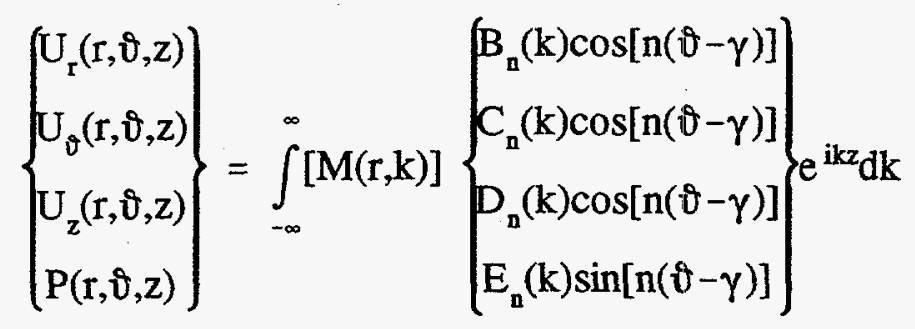

In (8), elements of the matrix, $M_{i j}, i, j=1,2,3,4$ are to be obtained from the relationships among $U_{r}, U_{\theta}$ $U_{z}$ and $P$, which are governed by the equations of motion. Coefficients $B_{n}, C_{n}, D_{n}$ and $E_{n}$, together with $A_{n}$ in equation (4), must be determined by the boundary conditions.

The equations of motion for poroelastic medium in cylindrical coordinates are given by ${ }^{[3]}$

$$
\begin{array}{r}
\mu\left[\nabla^{2} U_{r}-\frac{1}{r}\left(\frac{2}{r} \frac{\partial U_{\theta}}{\partial \theta}+\frac{U_{r}}{r}\right)\right]+(\lambda+\mu) \frac{\partial \Delta}{\partial r}+\omega^{2} \bar{\rho} U_{r}-\bar{\alpha} \frac{\partial P}{\partial r}=0 \\
\mu\left[\nabla^{2} U_{\theta}-\frac{1}{r}\left(-\frac{2}{r} \frac{\partial U_{r}}{\partial \theta}+\frac{U_{\theta}}{r}\right)\right]+(\lambda+\mu) \frac{1}{r} \frac{\partial \Delta}{\partial \theta}+\omega^{2} \bar{\rho} U_{\theta}-\frac{\bar{\alpha}}{r} \frac{\partial P}{\partial \theta}=0 \\
\mu \nabla^{2} U_{z}+(\lambda+\mu) \frac{\partial \Delta}{\partial z}+\omega^{2} \bar{\rho} U_{z}-\bar{\alpha} \frac{\partial P}{\partial z}=0 \\
-\bar{\alpha} \Delta+\theta \nabla^{2} \mathrm{P}-\beta P=0
\end{array}
$$

where 


$$
\begin{aligned}
\nabla^{2} & =\frac{\partial^{2}}{\partial r^{2}}+\frac{1}{r} \frac{\partial}{\partial r}+\frac{1}{r^{2}} \frac{\partial^{2}}{\partial \vartheta}+\frac{\partial^{2}}{\partial z^{2}} \\
\Delta & =\frac{\partial U_{r}}{\partial r}+\frac{U_{r}}{r}+\frac{1}{r} \frac{\partial U_{\vartheta}}{\partial \vartheta}+\frac{\partial U_{z}}{\partial z}
\end{aligned}
$$

Next, we introduce $\left\{U_{1 n} U_{2 n} U_{3 n} P_{n}\right\}^{\mathrm{T}}$ through the following expression

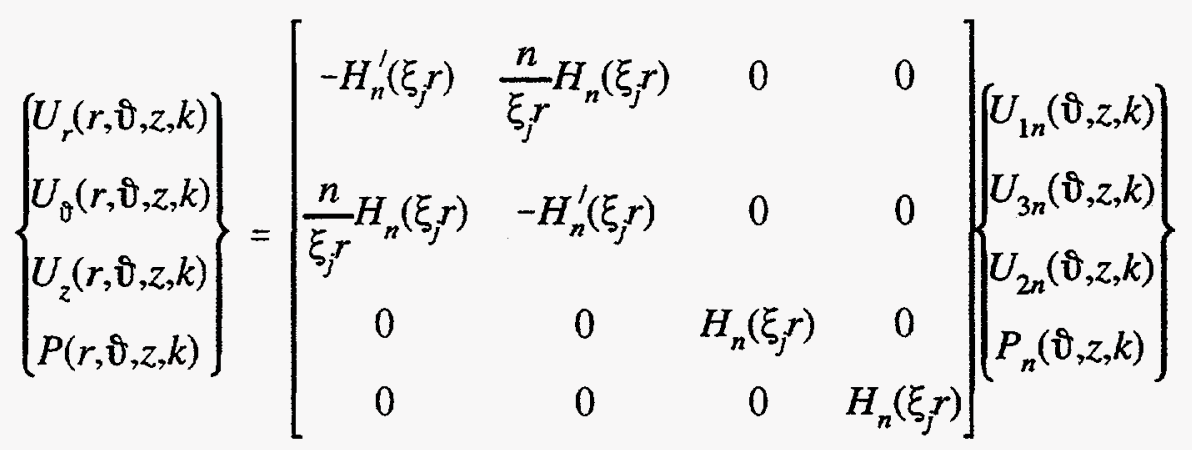

Upon applying the following differentiation,

$$
\begin{aligned}
\frac{\partial}{\partial z} & =i k \\
\frac{\partial^{2}}{\partial \theta^{2}} & =-n^{2} \\
\frac{\partial}{\partial \theta} & =-n \quad j=1,2,3 \\
& =n \quad j=4 \\
\frac{\partial^{2}}{\partial r^{2}}+\frac{1}{r} \frac{\partial}{\partial r} & =\frac{n^{2}}{r^{2}}-\xi_{j}^{2}
\end{aligned}
$$

Equation (9) can be rewritten as

$$
\begin{array}{r}
\mu \nabla_{n \pm 1}^{2}\left( \pm U_{1 n}+U_{3 n}\right)+(\lambda+\mu)\left(\frac{\partial \Delta_{n}}{\partial r} \mp \frac{n}{r} \Delta_{n}\right)+\omega^{2} \bar{\rho}\left( \pm U_{1 n}+U_{3 n}\right)-\bar{\alpha}\left(\frac{\partial}{\partial r} \mp \frac{n}{r}\right) P_{n}=0 \\
\mu \nabla^{2} U_{2 n}+(\lambda+\mu) \frac{\partial \Delta_{n}}{\partial z}+\omega^{2} \bar{\rho} U_{2 n}-\bar{\alpha} \frac{\partial P_{n}}{\partial z}=0 \\
-\bar{\alpha} \Delta_{n}+\theta \nabla^{2} P_{n}-\beta P_{n}=0
\end{array}
$$

where 


$$
\begin{aligned}
& \nabla_{n}^{2}=\frac{\partial^{2}}{\partial r^{2}}+\frac{1}{r} \frac{\partial}{\partial r}-\frac{n^{2}}{r^{2}}+\frac{\partial^{2}}{\partial z^{2}} \\
& \Delta_{n}=\frac{\partial U_{r}}{\partial r}+\frac{U_{r}}{r}+\frac{n}{r} U_{\vartheta}+\frac{\partial U_{z}}{\partial z}
\end{aligned}
$$

It can be seen that equation (13) yields

$$
[m]\left\{U_{1 n} U_{2 n} P_{n}\right\}^{T}=\left[\begin{array}{lll}
0 & 0 & 0
\end{array}\right\}^{T}
$$

where

$$
\begin{array}{ccc}
m_{11}=-(\lambda+2 \mu) \xi_{j}^{2}-\mu k^{2}+\omega^{2} \bar{\rho} & m_{12}=-i(\lambda+\mu) k \xi_{j} & m_{13}=\bar{\alpha} \xi_{j} \\
m_{21}=-i(\lambda+\mu) k \xi_{j} & m_{22}=(\lambda+2 \mu) k^{2}+\mu \xi_{j}^{2}-\omega^{2} \bar{\rho} & m_{23}=i k \bar{\alpha} \\
m_{31}=\bar{\alpha} \xi_{j} & m_{32}=i k \bar{\alpha} & m_{33}=\theta k^{2}+\theta \xi_{j}^{2}+\beta
\end{array}
$$

for $j=1,2,3$ and

$$
\left[\mu\left(k^{2}+\xi_{4}^{2}\right)-\omega^{2} \bar{\rho}\right] U_{3 n}=0
$$

Equations (15-17) can be used to obtain the radial wavenumbers given in (7). Equations (1516) also govern the relationship among $\left\{U_{1 n} U_{2 n} P_{n}\right\}^{\mathrm{T}}$. Denote $U_{1 n} / P_{n}$ and $U_{2 n} / P_{n}$ by $R_{j}$, and $Q_{j}$ for $j=1,2$, and $U_{1 n} / U_{2 n}$ and $P_{n} / U_{2 n}$ by $R_{j}$, and $Q_{j}$, for $j=3$, respectivelly. Then we have

$$
\begin{aligned}
& R_{j}(k)=-\frac{m_{13} m_{22}-m_{12} m_{23}}{m_{11} m_{22}-m_{12} m_{21}} \\
& Q_{j}(k)=-\frac{m_{11} m_{23}-m_{13} m_{21}}{m_{11} m_{22}-m_{12} m_{21}} \\
& R_{3}(k)=-\frac{m_{12} m_{33}-m_{13} m_{32}}{m_{11} m_{33}-m_{31} m_{13}} \\
& Q_{3}(k)=-\frac{m_{11} m_{32}-m_{31} m_{12}}{m_{11} m_{33}-m_{31} m_{13}}
\end{aligned}
$$

Equations (14) and (16) imply that 


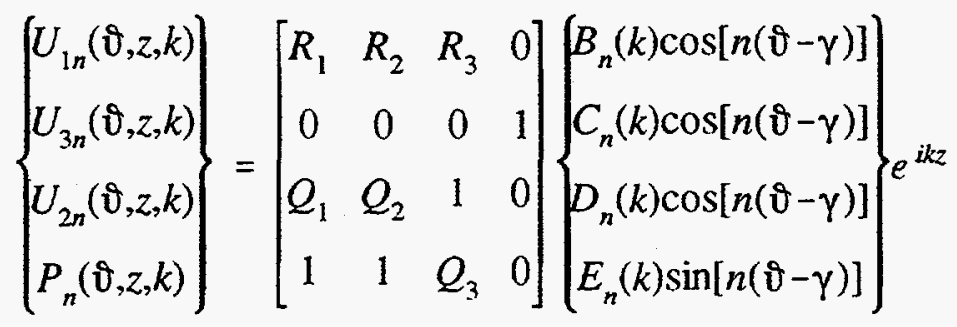

and, with the aid of equations (8) and (11),

$$
[M(r, k)]=\left[\begin{array}{cccc}
-H_{n}^{\prime}\left(\xi_{1} r\right) R_{1} & -H_{n}^{\prime}\left(\xi_{2} r\right) R_{2} & -H_{n}^{\prime}\left(\xi_{3} r\right) R_{3} & \frac{n}{\xi_{4} r} H_{n}\left(\xi_{4} r\right) \\
\frac{n}{\xi_{1} r} H_{n}\left(\xi_{1} r\right) R_{1} & \frac{n}{\xi_{2} r} H_{n}\left(\xi_{2} r\right) R_{2} & \frac{n}{\xi_{3} r} H_{n}\left(\xi_{3} r\right) R_{3} & -H_{n}^{\prime}\left(\xi_{4} r\right) \\
H_{n}\left(\xi_{1} r\right) Q_{1} & H_{n}\left(\xi_{2} r\right) Q_{2} & H_{n}\left(\xi_{3} r\right) & 0 \\
H_{n}\left(\xi_{1} r\right) & H_{n}\left(\xi_{2} r\right) & H_{n}\left(\xi_{3} r\right) Q_{3} & 0
\end{array}\right]
$$

Finally, the stress and volumetric flux components that appear in the boundary conditions include $\tau_{r r}, \tau_{r \theta} \tau_{r z}$ and $w_{r}$. Their Fourier transformed counterparts, $T_{r r}, T_{r \theta}, T_{r z}$ and $W_{r}$, are related to $U_{r}, U_{\theta}, U_{z}$ and $P$ through $^{[3]}$

$$
\begin{aligned}
T_{r r} & =2 \mu \frac{\partial U_{r}}{\partial r}+\lambda \Delta-\bar{\alpha} P \\
T_{r \vartheta} & =\mu\left(\frac{1}{r} \frac{\partial U_{r}}{\partial \vartheta}+\frac{\partial U_{\vartheta}}{\partial r}-\frac{U_{\vartheta}}{r}\right) \\
T_{r z} & =\mu\left(\frac{\partial U_{z}}{\partial r}+\frac{\partial U_{r}}{\partial z}\right) \\
W_{r} & =\theta\left(\omega^{2} \rho_{f} U_{r}-\frac{\partial P}{\partial r}\right)
\end{aligned}
$$

Denote $T_{r} / U_{r}, T_{r \theta} / U_{r}, T_{r z} / U_{r}$ and $W_{r} / U_{r}$ by $T_{j}, U_{j}, W_{j}$ and $X_{j}, j=1,2,3,4$. Then equations (11, $20,21)$ together provide the following relationships for these four ratios: 


$$
\begin{aligned}
T_{j}(r, k) & =(\lambda+2 \mu) \frac{\partial M_{1 j}}{\partial r}+\frac{\lambda}{r} M_{1 j} \mp \frac{n \lambda}{r} M_{2 j}+i \lambda k M_{3 j}-\bar{\alpha} M_{4 j} \\
U_{j}(r, k) & =\mp \frac{n \mu}{r} M_{1 j}-\frac{\mu}{r} M_{2 j}+\mu \frac{\partial M_{2 j}}{\partial r} \\
W_{j}(r, k) & =\mu \frac{\partial M_{3 j}}{\partial r}+i \mu k M_{1 j} \\
X_{j}(r, k) & =\theta \omega^{2} \rho_{f} M_{1 j}-\theta \frac{\partial M_{4 j}}{\partial r}
\end{aligned}
$$

where

$$
\begin{array}{rlrl}
\frac{\partial M_{1 j}}{\partial r} & =-R_{j}(k) \xi_{j} H_{n}^{\prime \prime}\left(\xi_{j} r\right) & j=1,2,3 \\
& =-\frac{n}{\xi_{4} r^{2}} H_{n}\left(\xi_{4} r\right)+\frac{n}{r} H_{n}^{\prime}\left(\xi_{4} r\right) & j=4 \\
\frac{\partial M_{2 j}}{\partial r} & =-\frac{n}{\xi_{j}{ }^{2}} R H_{n}\left(\xi_{j} r\right)+\frac{n}{r} R_{j}(k) H_{n}^{\prime}\left(\xi_{j} r\right) & j=1,2,3 \\
& =-\xi_{4} H_{n}^{\prime \prime}\left(\xi_{4} r\right) & & j=4 \\
\frac{\partial M_{3 j}}{\partial r} & =\xi_{j} H_{n}^{\prime}\left(\xi_{j} r\right) Q_{j} & & \\
& =\xi_{3} H_{n}^{\prime}\left(\xi_{3} r\right) & & \\
& =0 & & j=1,2 \\
\frac{\partial M_{4 j}}{\partial r} & =\xi_{j} H_{n}^{\prime}\left(\xi_{j} r\right) & & \\
& =\xi_{3} H_{n}^{\prime}\left(\xi_{3} r\right) Q_{3} & j=1,2 & \\
& =0 & j=3 & \\
& 0 & &
\end{array}
$$

Solution for the open-pore boundary conditions

The open-pore boundary conditions are defined as ${ }^{[4]}$

$$
\begin{aligned}
{\left[-U_{r r}(r, \theta, z)+U_{r}(r, \theta, z)+\right.} & \left.W_{r}(r, \theta, z)\right]_{r=a}=0 \\
{\left[(1-\phi) P_{f}(r, \theta, z)+T_{r r}(r, \theta, z)\right]_{r=a} } & =0 \\
{\left[-P_{f}(r, \theta, z)+P(r, \theta, z)\right]_{r=a} } & =0 \\
{\left[T_{r \theta}(r, \theta, z)\right]_{r=a} } & =0 \\
{\left[T_{r z}(r, \theta, z)\right]_{r=a} } & =0
\end{aligned}
$$


where $a$ is the radius of the borehole and $\phi$ the porosity of the formation.

Using notations $M_{i j}, T_{j}, U_{j}, W_{j}$ and $X_{j}, i, j=1,2,3,4$, Eqs. $(2-4,8,20,24)$ lead to

$$
\left[\begin{array}{ccccc}
-U_{r f}^{R} & M_{11}+X_{1} & M_{12}+X_{2} & M_{13}+X_{3} & M_{14}+X_{4} \\
(1-\phi) P_{f}^{R} & T_{1} & T_{2} & T_{3} & T_{4} \\
-P_{f}^{R} & M_{41} & M_{42} & M_{43} & M_{44} \\
0 & U_{1} & U_{2} & U_{3} & U_{4} \\
0 & W_{1} & W_{2} & W_{3} & W_{4}
\end{array}\right]_{=a} \quad\left\{\begin{array}{c}
A_{n}(k) \\
B_{n}(k) \\
C_{n}(k) \\
D_{n}(k) \\
E_{n}(k)
\end{array}\right\}=\left\{\begin{array}{c}
U_{r f}^{0} \\
-(1-\phi) P_{f}^{0} \\
P_{f}^{0} \\
0 \\
0
\end{array}\right\}_{r=a}
$$

where

$$
\begin{gathered}
U_{r f}^{0}=-\frac{i}{4 n !}\left(\frac{\xi_{f} r_{0}}{2}\right)^{n} \epsilon_{n} \xi_{f} H_{n}^{\prime}\left(\xi_{f} a\right) \\
U_{r f}^{R}=-\frac{i}{4 n !}\left(\frac{\xi_{f} f_{0}}{2}\right)^{n} \epsilon_{n} \xi_{f} J_{n}^{\prime}\left(\xi_{f} a\right) \\
P_{f}^{0}=-\frac{i}{4 n !}\left(\frac{\xi_{f} r_{0}}{2}\right)^{n} \epsilon_{n} \omega^{2} \rho_{f} H_{n}\left(\xi_{j} a\right) \\
P_{f}^{R}=-\frac{i}{4 n !}\left(\frac{\xi_{f} r_{0}}{2}\right)^{n} \epsilon_{n} \omega^{2} \rho_{f} J_{n}\left(\xi_{f} a\right)
\end{gathered}
$$

Upon solving equation (25) for $A_{n}, B_{n}, C_{n}, D_{n}$ and $E_{n}$, the responses in the borehole and formation can be obtained from equations (1) and (8).

Solution for the closed-pore boundary conditions

The closed-pore boundary conditions are defined as ${ }^{[4]}$

$$
\begin{aligned}
{\left[-U_{r r}(r, \theta, z)+U_{r}(r, \theta, z)\right]_{r=a} } & =0 \\
{\left[U_{f}(r, \theta, z)+U_{r r}(r, \theta, z)-\phi P(r, \theta, z)\right]_{r=a} } & =0 \\
{\left[W_{r}(r, \theta, z)\right]_{r=a} } & =0 \\
{\left[T_{r \theta \theta}(r, \theta, z)\right]_{r=a} } & =0 \\
{\left[T_{r z}(r, \theta, z)\right]_{r=a} } & =0
\end{aligned}
$$


Similar to the open-pore case, equations $(2-4,8,20,27)$ lead to

$$
\left[\begin{array}{ccccc}
-U_{r f}^{R} & M_{11} & M_{12} & M_{13} & M_{14} \\
P_{f}^{R} & T_{1}-\phi M_{41} & T_{2}-\phi M_{42} & T_{3}-\phi M_{43} & T_{4}-\phi M_{44} \\
0 & X_{1} & X_{2} & X_{3} & X_{4} \\
0 & U_{1} & U_{2} & U_{3} & U_{4} \\
0 & W_{1} & W_{2} & W_{3} & W_{4}
\end{array}\right]_{=a} \quad\left\{\begin{array}{c}
A_{n}(k) \\
B_{n}(k) \\
C_{n}(k) \\
D_{n}(k) \\
E_{n}(k)
\end{array}\right\}=\left\{\begin{array}{c}
U_{r f}^{0} \\
-P_{f}^{0} \\
0 \\
0 \\
0
\end{array}\right\}_{r=a}
$$

This concludes the formulation of the response to an acoustic multipole in fluid-filled borehole surrounded by poroelastic formations.

\section{REFERENCES}

[1] D. P. Schmitt, "Acoustic Multipole Logging in Transversely Isotropic Poroelastic Formations", J. Acoust. Soc. Am. 86(6), 2397-2471, 1989.

[2] A. L. Kurkjian and S.-K. Chang, "Acoustic Multipole Sources in Fluid-Filled Boreholes", Geophysics, 51(1), 148-163, 1986.

[3] J. O. Parra, "Analysis of Elastic Wave Propagation in Stratified Fluid-Filled Porous Media for Interwell Seismic Applications", J. Acoust. Soc. Am., 90(5), 2557-2575, 1991.

[4] D. P. Schmitt, "Effects of Radial Layering When Logging in Saturated Porous Formations", $J$. Acoust. Soc. Am., 46(6), 2200-2213, 1988. 
B. Acoustic Waves in a Fluid-Filled Borehole Surrounded by a Transversely Isotropic Formation with a Horizontal Axis of Symmetry

\section{Introduction}

The above-titled study concerns the simulation and interpretation of sonic logging data acquired in a vertical, cylindrical, fluid-filled borehole, $r=a, 0<\theta<360^{\circ},-\infty<z<\infty$, which is surrounded by a formation with layers and fractures parallel to $z$-axis. The main interest lies in the detection and characterization of these layers and fractures. Assuming that the number of layers or fractures is large and the thicknesses of the layer or fractures are small compared to the radius of the borehole, then the surrounding medium may be represented by a transversely isotropic, viscoelastic unbounded medium with a horizontal axis of symmetry.

This report presents a novel approach, the integral transformed boundary integral equation (ITBIE) method, to solve this boundary value problem. In a traditional BIE approach, the displacements and pressure on the entire borehole surface constitute the basic unknowns and are coupled in three-dimensional boundary integral equations. In order to solve the BIEs numerically, the infinite cylindrical surface is discretized by boundary nodes, resulting in a linear system of equations whose coefficient matrix is determined from the Green's functions and associated stresses for every pair of sources (nodes) and receivers (Gaussian points) on this surface. These pairs will be called SR pairs in this report. The total number of nodes can be written as $N_{\theta} \mathrm{x} N_{z}$, where $N_{\theta}$ and $N_{z}$ are the number of nodes in $\theta$ and $z$ directions, respectively. The number of SR pairs equals to $N_{\theta} \times N_{z} \mathrm{x} N_{g}^{2}$, where $N_{g}$ is the number of Gaussian points divided by the number of nodes in an element. In the ITBIE approach, the above BIEs are integral-transformed, with the $z$-dependence being changed to vertical wavenumber dependence. As a result, the solution of the original problem is given in the form of a wavenumber integral which requires a solution of the transformed BIEs for each vertical wavenumber being sampled in the integration. The surface integrals of the original BIEs are then degenerated to line integrals. The dimension of the transformed linear system is reduced from the original one $\left(N_{\theta} \mathrm{x} N_{z}\right)$ to $N_{\theta}$ while all the coefficients are obtained from the Green's functions and associated stresses through integration with respect to $z$ from negative to positive infinity.

Compared to the traditional BIE method, the ITBIE method reduces the number of nodes by a factor of $N_{z}$ and the number of SR pairs by a factor of $N_{z} \mathrm{x} N_{g}$. In addition, the number of unknowns per node is reduced from six to four. But the ITBIE method requires an infinite $z$-integral to obtain the matrix for each vertical wavenumber and an infinite vertical wavenumber integral to yield a solution in the frequency domain. Overall, the benefits of the greatly reduced degree of freedom (DOF) undoubtedly surpasses the drawback of the two-folded integrals.

The major challenge in the computational aspects of this approach is the evaluation of integrals. The above mentioned integrations in the $z$ and $k_{z}$ domain are both infinite and oscillatory. The kernel of the latter possess spikes and other irregularities. The ITBIEs themselves consist of irregularly oscillatory integrals in the $\theta$ domain. In addition, for anisotropic formations, the fundamental solutions are in general in the form of radial wavenumber $\left(k_{\rho}\right)$ integrals where the radial direction is perpendicular to the axis of symmetry of the anisotropy. We shall show that the evaluation of $k_{\rho}$-integrals, as well as the $z$-integrals and $k_{z}$-integrals can be carried out accurately and 
efficiently by an advanced quadrature technique, the Modified Clenshaw-Curtis (MCC) method ${ }^{[1-3]}$. The $\theta$-integrals of the ITBIE are handled by a standard Gaussian quadrature.

In what follows, the analytical developments and numerical considerations for the above outlined approach are provided in details.

\section{Definition of the Model}

The borehole is bounded by the surface $r=a$ in the cylindrical coordinate system $(r, \theta, z)$. The borehole is full of fluid. An acoustic source (monopole or dipole) is located at the origin of the system. The exterior domain (formation) is a unbounded, uniform, viscoelastic, medium. For the ITBIE approach, a Cartesian coordinate system $(x, y, z)$, with $x$-axis coinciding with the $r$-axis at $\theta=0$ is also needed:

$$
\begin{aligned}
& x=r \cos \theta \\
& y=r \sin \theta
\end{aligned}
$$

Clearly, when the exterior medium is isotropic, the boundary value problem has a symmetry about the $z$-axis. When layers or fractures are present and parallel to the $y z$ plane, the problem can be seen to have symmetries about the $x z$ and $y z$ planes.

The boundary conditions are written as follows:

$$
\begin{aligned}
& \text { (A) continuity of radial displacement; } \\
& u_{r}^{b}(a, \theta, z)=u_{r}(a, \theta, z)
\end{aligned}
$$

where superscripts $b$ has been used to denote displacement of the fluid in the borehole. Displacement components of the formation do not carry a superscript.

(B) continuity of radial normal stress;

$$
-p(a, \theta, z)=\tau_{r}(a, \theta, z)
$$

where $p$ and $\sigma$ represent the pressure of fluid in the borehole and that in the pores of the saturated solid, respectively.

(C) vertical shear stress free;

$$
\tau_{r z}(a, \theta, z)=0
$$

and,

(D) tangential shear stress free. 


$$
\tau_{r z}(a, \theta, z)=0
$$

In the above, the angle $\theta$ ranges from 0 to $2 \pi$ radians.

Since the formation has an axis of symmetry in a horizontal direction, it is necessary to introduce another cylindrical system, $(\rho, \psi, \zeta)$, with origin at a boundary point and $\zeta$ the symmetry axis of the exterior medium. For the model concerned, the $\zeta$-axis is parallel to $x$-axis, $\rho$ axis parallel to $y$-axis at $\psi=0$.

\section{The Boundary Integral Equation (BIE) for Acousto-Elastic Interaction}

boundary integral equations ${ }^{[4]}$.

A general acousto-elastic interaction problem is governed by the following

(1) For the elastic domain:

$$
C u_{k}(x)=\int_{S}\left[G_{i k}(\xi, x) \tau_{i j}(\xi)-u_{i}(\xi) T_{i j}^{k}(\xi, x)\right] n_{j} d S(\xi)
$$

where $i, j, k=1,2,3$ and repeated indices imply summation.

(2) For the acoustic domain:

$$
C_{f} p(x)=\int_{S}\left[Q(\xi, x) \omega^{2} \sigma_{f} \mu(\xi)-\frac{\partial Q(\xi, x)}{\partial n} p(\xi)\right] d S(\xi)+p^{0}(x)
$$

In both Eqs. (6) and (7) the integration surface $S$ is the interface between the two domains, $n_{j}$ the direction cosine of its norm, and $\sigma_{f}$ the mass density of borehole fluid. In Equation (6), $u_{k}(\xi)$ and $\tau_{i j}(\xi)$ are the unknown displacement and stress components at a boundary field point, $\xi$, respectively. $G_{i k}(\xi, x)$ is the Green's function in an unbounded elastic medium with the properties of the host domain, which is defined as the displacement in the $i$-direction at $\xi$ due to a force in $k$-direction at the point $x . T_{i j}^{k}(\xi, x)$ is the corresponding stress tensor. In Equation (7), $p(\xi)$ is the unknown pressure of fluid at $\xi$. Notation $Q(\xi, x)$ represents the pressure at $\xi$ due to a unit acoustic source at $\boldsymbol{x}$ in the fluid medium in the absence of the host medium. And $p^{0}(\boldsymbol{x})$ is used to denote the pressure at $x$ due to the given acoustic source in an unbounded medium with the properties of the fluid domain.

Finally, coefficients $C$ and $C_{b}$ are constants depending on the location of the field point $x$. It is noted that Eqs. (6) and (7) contain a singularity when $x$ and $\xi$ coincides. The singularity associated with $T_{i j}{ }^{k}(\xi, x)$ is of the $1 /|\xi-x|$ type and can be interpreted by the Cauchy principal value. As a result, $C(x)$ and $C_{f}(x)$ equal one when $x$ is off the boundary and $1 / 2$ when it is on a smooth part of the boundary. The singularity associated with $G_{i k}\left(\xi_{x} x\right)$ is of the logarithmic type and is integrable. 
The unknown $u_{k}(\zeta)$ and $\tau_{i j}(\zeta)$ on $S$ must always be obtained first by solving the BIEs, with $C$ and $C_{f}$ being $1 / 2$ for the current model. Then $u_{k}(x)$ and $p(x)$ at any given field point $\boldsymbol{x}$ outside the boundary can be obtained through direct evaluation of the integrals in Eqs. (6-7), with $C$ and $C_{f}$ being 1 and $u_{k}(\xi), \tau_{i j}(\xi), p(\xi), \partial p(\xi) / \partial \boldsymbol{n}$ given on $S$.

\section{The Green's functions and Associated Stresses}

$Q(\xi, x)$ and $\partial Q(\xi, x) / \partial n$ for the fluid in the borehole required by Eqs. (6-7) can be found in standard text $[4]$ :

$$
\begin{aligned}
Q(\xi, x) & =\frac{e^{i k_{f} R}}{4 \pi R} \\
\frac{\partial Q(\xi, x)}{\partial n} & =\left(-1+i k_{j} R\right)\left(\xi_{j}-x_{j}\right) n_{j} \frac{e^{i k_{j} R}}{4 \pi R^{3}}
\end{aligned}
$$

where $k_{f}=\omega / V_{f} \omega$ is the circular frequency, $V_{f}$ is the acoustic wave speed in water, and $R=$ $\left(x_{1}^{2}+x_{2}^{2}+x_{3}^{2}\right)^{1 / 2}$.

$$
G_{i k}(\xi, x) \text { and } T_{i j}^{k}(\xi, x) \text { for an isotropic formation can also be found in a standard text [4]: }
$$

$$
\begin{aligned}
G_{i j}(\xi, x) & =\frac{1}{4 \pi \sigma \omega^{2}}\left[k_{s}^{2} \frac{e^{i k_{s} R}}{R} \delta_{i j}-\frac{\partial^{2}}{\partial x_{i} \partial x_{j}} \frac{e^{i k_{p} R}-e^{i k_{s} R}}{R}\right] \\
T_{i j}^{k}(\xi, x) & =\lambda \delta_{i j} G_{m k, m}(\xi, x)+\mu\left[G_{i k, j}(\xi, x)+G_{j k, l}(\xi, x)\right]
\end{aligned}
$$

where $\lambda, \mu$ are Lamés elastic constants, $\sigma$ is the mass density, $k_{p}=\omega / V_{p}, k_{s}=\omega / V_{s}$, and $V_{p}$ and $V_{s}$ are the $P$ - and $S$-wave velocities in the formation.

isotropic (TI) formulation.

The above expressions are used as a special case to test the transversely

The response of a TI medium to a force is well established and documented ${ }^{[5]}$. $G_{i k}(\xi, x)$ and $T_{i j}{ }^{k}(\xi, x)$ are in general expressed in terms of the following wavenumber integral:

$$
I(\rho, \Psi, \zeta)=\int_{0}^{\infty} \sum_{m=0}^{2} F_{m}\left(k_{\rho}, \zeta\right) \Psi_{m}(\psi) J_{m}\left(k_{\rho} \rho\right) d k_{\rho}
$$

where $k_{\rho}$ is the wavenumber in $\rho$ direction, and $J_{m}\left(k_{\rho} \rho\right)$ is the Bessel function of the $m$-th order. $\Psi_{m}(\psi)$ denotes $\sin \psi$ or $\cos \psi$.

Specifically, the displacements and stresses in $(\rho, \psi, \zeta)$ are given by 


$$
\begin{aligned}
& \left\{\begin{array}{l}
u_{\rho} \\
u_{\phi} \\
u_{\zeta}
\end{array}\right\}=\int_{0}^{\infty}\left[\begin{array}{ccccc}
J_{1}\left(k_{\rho} \rho\right) & 0 & J_{1}^{\prime}\left(k_{\rho} \rho\right) \cos \phi & 0 & -J_{1}\left(k_{\rho} \rho\right) \cos \phi /\left(k_{\rho} \rho\right) \\
0 & 0 & -J_{1}\left(k_{\rho} \rho\right) \sin \phi /\left(k_{\rho} \rho\right) & 0 & \left.J_{1}^{\prime} k_{\rho} \rho\right) \sin \phi \\
0 & J_{0}\left(k_{\rho} \rho\right) & 0 & J_{1}\left(k_{\rho} \rho\right) \cos \phi & 0
\end{array}\right]
\end{aligned}
$$

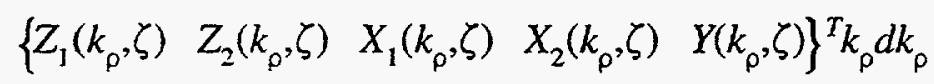

and

$$
\left\{\begin{array}{l}
\sigma_{\rho \rho} \\
\sigma_{\phi \phi} \\
\sigma_{\zeta \zeta} \\
\sigma_{\phi \zeta} \\
\sigma_{\rho \zeta} \\
\sigma_{\rho \phi}
\end{array}\right\}=\left[\begin{array}{ccc}
C_{11} \frac{\partial}{\partial \rho}+\frac{C_{12}}{\rho} & C_{12} \frac{1}{\rho} \frac{\partial}{\partial \phi} & C_{13} \frac{\partial}{\partial \zeta} \\
C_{12} \frac{\partial}{\partial \rho}+\frac{C_{11}}{\rho} & C_{11} \frac{1}{\rho} \frac{\partial}{\partial \phi} & C_{13} \frac{\partial}{\partial \zeta} \\
C_{13} \frac{\partial}{\partial \rho}+\frac{C_{13}}{\rho} & C_{13} \frac{1}{\rho} \frac{\partial}{\partial \phi} & C_{33} \frac{\partial}{\partial \zeta} \\
0 & C_{44} \frac{\partial}{\partial \zeta} & C_{44} \frac{1}{\rho} \frac{\partial}{\partial \phi} \\
C_{44} \frac{\partial}{\partial \zeta} & 0 & C_{44} \frac{\partial}{\partial \rho} \\
C_{66} \frac{1}{\rho} \frac{\partial}{\partial \phi} & C_{66}\left(\frac{\partial}{\partial \rho}-\frac{1}{\rho}\right) & 0
\end{array}\right]\left\{\begin{array}{l}
u_{\rho} \\
u_{\phi} \\
u_{\zeta}
\end{array}\right\}
$$

where $Z_{1}$ and $Z_{2}$ are the horizontal and vertical displacement kernels due to a unit vertical force in the P-SV part of the three-dimensional motion, $X_{1}$ and $X_{2}$ are the horizontal and vertical displacement kernels due to a unit horizontal force in the P-SV part, and $Y$ is the anti-plane displacement kernel due to a unit anti-plane force in the SH part. The expressions of $Z_{1}, Z_{2}, X_{1}, X_{2}$ and $Y$ are given in Appendix A.

After some algebra, we obtain the following expressions: 


$$
\begin{aligned}
u_{\rho}(\rho, \phi, \zeta)= & -\frac{\rho}{2}\left(I_{1}+I_{2}\right)+\frac{1}{2}\left(I_{7}-I_{8}-I_{16}-I_{17}\right) \cos \phi \\
u_{\phi}(\rho, \phi, \zeta)= & \frac{1}{2}\left(-I_{7}-I_{8}+I_{16}-I_{17}\right) \sin \phi \\
u_{\zeta}(\rho, \phi, \zeta)= & I_{4}+\frac{\rho}{2}\left(I_{12}+I_{13}\right) \cos \phi \\
\tau_{\rho \rho}(\rho, \phi, \zeta)= & C_{12} I_{1}+\frac{1}{2}\left(C_{11}-C_{12}\right)\left(I_{1}-I_{2}\right) \\
& +C_{13} I_{6}+\left[-C_{12} I_{9}+\left(C_{11}-C_{12}\right)\left(-I_{9}+I_{8} / \rho\right)+C_{13} I_{14}+\left(C_{11}-C_{12}\right) I_{17} / \rho\right] \cos \phi \\
\tau_{\phi \phi}(\rho, \phi, \zeta)= & C_{11} I_{1}+\frac{1}{2}\left(C_{12}-C_{11}\right)\left(I_{1}-I_{2}\right) \\
& +C_{13} I_{6}+\left[-C_{11} I_{9}+\left(C_{12}-C_{11}\right)\left(-I_{9}+I_{8} / \rho\right)+C_{13} I_{14}+\left(C_{12}-C_{11}\right) I_{17} / \rho\right] \cos \phi \\
\tau_{\zeta \zeta}(\rho, \phi, \zeta)= & C_{13} I_{1}+C_{33} I_{6}+\left(-C_{13} I_{9}+C_{33} I_{14}\right) \cos \phi \\
\tau_{\phi \zeta}(\rho, \phi, \zeta)= & \frac{C_{44}}{2}\left(-I_{10}-I_{11}-I_{12}-I_{13}+I_{18}-I_{19}\right) \sin \phi \\
\tau_{\rho \zeta}(\rho, \phi, \zeta)= & C_{44}\left(I_{3}-I_{5}\right)+\frac{C_{44}}{2}\left(I_{10}-I_{11}+I_{12}-I_{13}-I_{18}-I_{19}\right) \cos \phi \\
\tau_{\rho \phi}(\rho, \phi, \zeta)= & C_{66}\left(2 I_{8} / \rho+2 I_{17} / \rho-I_{15}\right) \sin \phi
\end{aligned}
$$

where $I_{m}(\rho, \zeta), m=1,2 \ldots .19$ are $k_{\rho}$-integrals. Their definitions are given in Appendix B.

Finally, in order to obtain the Green's functions and associated stresses in Cartesian coordinates $(x, y, z)$, we need the transformation defined below:

$$
\left\{\begin{array}{l}
u_{x} \\
u_{y} \\
u_{z}
\end{array}\right\}=\left[\begin{array}{ccc}
\cos \phi & -\sin \phi & 0 \\
\sin \phi & \cos \phi & 0 \\
0 & 0 & 1
\end{array}\right]\left\{\begin{array}{l}
u_{\rho} \\
u_{\phi} \\
u_{\zeta}
\end{array}\right\}
$$

$$
\left\{\begin{array}{l}
\tau_{x x} \\
\tau_{y y} \\
\tau_{z z} \\
\tau_{y z} \\
\tau_{x z} \\
\tau_{x y}
\end{array}\right\}\left\{\begin{array}{cccccc}
\cos ^{2} \phi & \sin ^{2} \phi & 0 & 0 & 0 & -\sin 2 \phi \\
\sin ^{2} \phi & \cos ^{2} \phi & 0 & 0 & 0 & \sin 2 \phi \\
0 & 0 & 1 & 0 & 0 & 0 \\
0 & 0 & 0 & \cos \phi & \sin \phi & 0 \\
0 & 0 & 0 & -\sin \phi & \cos \phi & 0 \\
\frac{1}{2} \sin 2 \phi & -\frac{1}{2} \sin 2 \phi & 0 & 0 & 0 & \cos 2 \phi
\end{array}\right]\left\{\begin{array}{c}
\tau_{\rho \rho} \\
\tau_{\phi \phi} \\
\tau_{\zeta \zeta} \\
\tau_{\phi \zeta} \\
\tau_{\rho \zeta} \\
\tau_{\rho \phi}
\end{array}\right\}
$$




\section{The Integral Transformed Boundary Integral Equation Approach}

First, the BIEs on the cylindrical surface, $r=a, 0^{\circ}<\theta<360^{\circ},-\infty<z<\infty$, are converted to the BIEs on a circle, $r=a, 0^{\circ}<\theta<360^{\circ}$ in the $k_{z}$ domain through an integral transform of the unknowns with respect to $z$. Its inverse transform is given by

$$
\left\{\begin{array}{l}
u_{1}(r, \theta, z) \\
u_{2}(r, \theta, z) \\
u_{3}(r, \theta, z) \\
p(r, \theta, z)
\end{array}\right\}=\int_{-\infty}^{\infty}\left\{\begin{array}{l}
U_{1}\left(r, \theta, k_{z}\right) \\
U_{2}\left(r, \theta, k_{z}\right) \\
U_{3}\left(r, \theta, k_{z}\right) \\
P\left(r, \theta, k_{z}\right)
\end{array}\right\} e^{i k z} d k_{z}
$$

where $k_{z}$ is the vertical wavenumber. The BIEs (6-7) have been integral transformed and rewritten as

$$
C U_{k}\left(\theta_{0}\right)=-\int_{0}^{2 \pi}\left[g_{k}\left(\theta, \theta_{0}\right) P(\theta)+t_{k i}\left(\theta, \theta_{0}\right) U_{i}(\theta)\right] d \theta
$$

and

$$
C_{b} P\left(\theta_{0}\right)=\int_{0}^{2 \pi}\left[q\left(\theta, \theta_{0}\right) U_{i}(\theta) n_{i}(\theta)-q^{\prime}\left(\theta, \theta_{0}\right) P(\theta)\right] d \theta+P^{0}\left(\theta_{0}\right)
$$

where $i, k=1,2,3$. Note that $U_{i}$ or $U_{k}$ and $P$ are the unknown functions to be solved and $g_{k}, t_{k i}, q$ and $q^{\prime}$ denote $z$-integrals of given fundamental solutions $G_{i k}\left(\theta, \theta_{0}, z-z_{0}\right), T_{i j}^{k}\left(\theta, \theta_{0}, z-z_{0}\right), Q\left(\theta, \theta_{0}, z-z_{0}\right)$ and $\partial Q\left(\theta, \theta_{0}, z-z_{0}\right) / \partial r$ (see Section 4 for details):

$$
\begin{aligned}
& g_{k}\left(\theta, \theta_{0}\right)=\int_{-\infty}^{\infty} e^{i k_{z}\left(z-z_{0}\right)} G_{i k}\left(\theta, \theta_{0}, z-z_{0}\right) n_{i} d\left(z-z_{0}\right) \\
& t_{k i}\left(\theta, \theta_{0}\right)=\int_{-\infty}^{\infty} e^{i k_{z}\left(z-z_{0}\right)} T_{i j}^{k}\left(\theta, \theta_{0}, z-z_{0}\right) n_{j} d\left(z-z_{0}\right) \\
& q\left(\theta, \theta_{0}\right)=\int_{-\infty}^{\infty} e^{i k_{z}\left(z-z_{0}\right)} \omega^{2} \sigma \rho\left(\theta, \theta_{0}, z-z_{0}\right) d\left(z-z_{0}\right) \\
& q^{\prime}\left(\theta, \theta_{0}\right)=\int_{-\infty}^{\infty} e^{i k_{2}\left(z-z_{0}\right)} \frac{\partial Q}{\partial n}\left(\theta, \theta_{0}, z-z_{0}\right) d\left(z-z_{0}\right)
\end{aligned}
$$


As a result, the number of SR pairs becomes $N_{\theta} \mathrm{x} N_{g}$ after integral transform, which is $N_{z} \mathrm{x} N_{g}$ smaller than the original.

\section{The MCC Integration Method}

The MCC integration technique, co-developed by one of the authors ${ }^{i 1-31}$, is aimed at integrals of the type

$$
I=\int_{a}^{b} f(x) e^{i p x} d x
$$

and the type

$$
I=\int_{a}^{b} f(x) J_{n}(p x) d x
$$

where $p$ is a constant and $J_{n}$ denotes the Bessel function of the $n$th order. In this method, the kernel $f(x), a<x<b$, is fitted by the Chebyschev polynomials $T_{k}(\tau),-1<\tau<1, k=1,2 \ldots N$, through a change of variable, $x=(b-a) \tau / 2+(a+b) / 2$. After some algebra, the following quadrature rule is obtained for the integral in (20).

$$
I=\sum_{k=0}^{N}{ }_{m=0}^{N} \sum_{m}^{\prime \prime} a_{m}^{k} f\left(\frac{b-a}{2} \cos \frac{m \pi}{N}+\frac{a+b}{2}\right)
$$

where

$$
\begin{aligned}
& a_{m}^{k}=\frac{b-a}{4 N} \sum_{k=0}^{N} \cos \frac{m k \pi}{N} I_{k}(p, a, b) \\
& I_{k}(p, a, b)=\int_{-1}^{1} T_{k}(\tau) e^{i p\left(\frac{b-a}{2} \tau+\frac{a+b}{2}\right)} d \tau
\end{aligned}
$$

and a double prime implies that the first and last term of the sum are to be halved. It should be pointed out that $I_{k}$ in (23) can be evaluated analytically. A quadrature rule for the integral in (21) can be derived in a similar manner ${ }^{[1-3]}$.

The above integration technique has several important features. First, only the kernel $f(x)$ is fitted while the regularly oscillatory part $\exp (i p x)$ is present solely in $I_{k}$ of (23). This undoubtedly results in much fewer sampling points. Second, for any values of the constant, $p$, the sampling points of $f(x)$ remain the same and the variation of $p$ affects $I_{k}$ only. This is especially beneficial when the calculation of $f(x)$ is demanding and when a large number of integrals (20) with the same $f(x)$ and different $p$ are needed. Third, the unique properties of the Chebyschev polynomials allow the 
implementation of an adaptive procedure such that the distribution of sampling points is properly adjusted according to the irregularity of the kernel and that none of the sampling points generated in any step is wasted ${ }^{[1]}$. Finally, the quadrature rule of (22) has been extended to the case that $a$ or $b$ becomes infinity ${ }^{[2]}$. Unlike most integration methods in which the integral is truncated at a finite value of $x$, the MCC technique perform the integration of the infinite part completely and analytically. Clearly, the above features make the MCC technique an ideal quadrature tool for wavenumber integrals.

\section{Advantages of Using MCC Integration Method in the ITBIE Solution}

The above sections have indicated that the ITBIE approach consists of the evaluation of three-field integrals (16), (19), (10). It can be seen that the quadrature of all three integrals can be improved with the MCC technique. In particular, although the integral in (16) requires a series values of $k_{z}$, each of which needs an evaluation of the integral in (19), the sampling of the kernel of (19) remains the same. Therefore, the effort to obtain the integral in (19) for a large number of $k_{z}$ is nearly the same as a single evaluation of the integral. Similarly, the effort to evaluate the integral in (16) for a number of detectors with different values of $z$ and the integral in (10) for a number of $\zeta$ is nearly the same as a single evaluation of the integrals. In view of all these facts, the three-fold integration can be made a feasible task by the use of the MCC technique.

\section{8. $\quad z$-Integration of the Transformed BIE}

Consider the $z$-integrals in Equation (19). The kernel as a function of $z$ has a peak at $z=0$ and decays monotonically as $|z|$ approaches infinity. The curve fitting of this kernel is quite easy, especially with the MCC adaptive scheme. The regularly oscillating factor has been taken care

of analytically by the MCC formula. The infinite tail is truncated at a large value of $z$ in the current version of the computer program. The following far-field expression of the Green's

functions in isotropic media indicate the behavior of the kernel at large $z\left(=x_{3}\right)$, which is believed to be similar in the anisotropic case.

$$
G_{i j}=\frac{1}{4 \pi \sigma R}\left[\frac{e^{i k_{s} R}}{V_{s}^{2}}\left(\delta_{i j}-\frac{x_{l} x_{j}}{R^{2}}\right)+\frac{e^{i k_{p} R}}{V_{p}^{2}} \frac{x_{i} x_{j}}{R^{2}}\right]
$$

\section{From ITBIE to Linear Equation System}

In order to solve the Boundary Integral Equations (BIE's) numerically, they are usually converted into a system of linear equations as follows.

First, BIEs of the form of Eqs. (6-7) hav been rewritten as 


$$
C U_{k}\left(\theta_{0}\right)=-\int_{0}^{2 \pi}\left[g_{k}\left(\theta, \theta_{0}\right) P(\theta)+t_{k i}\left(\theta, \theta_{0}\right) U_{i}(\theta)\right] d \theta
$$

and

$$
C_{b} P\left(\theta_{0}\right)=\int_{0}^{2 \pi}\left[q\left(\theta, \theta_{0}\right) U_{i}(\theta) n_{i}(\theta)-q^{\prime}\left(\theta, \theta_{0}\right) P(\theta)\right] d \theta+P^{0}\left(\theta_{0}\right)
$$

where $i, k=1,2,3, r_{0}=a$ is removed from the parameters in parentheses, and

$$
\begin{aligned}
& g_{k}\left(\theta, \theta_{0}\right)=\int_{-\infty}^{\infty} e^{i k_{z}\left(z-z_{0}\right)} G_{i k}\left(\theta, \theta_{0}, z-z_{0}\right) n_{i} d\left(z-z_{0}\right) \\
& t_{k i}\left(\theta, \theta_{0}\right)=\int_{-\infty}^{\infty} e^{i k_{z}\left(z-z_{0}\right)} T_{i j}^{k}\left(\theta, \theta_{0}, z-z_{0}\right) n_{j} d\left(z-z_{0}\right) \\
& q\left(\theta, \theta_{0}\right)=\int_{-\infty}^{\infty} e^{i k_{z}\left(z-z_{0}\right)} \omega^{2} \sigma_{f} Q\left(\theta, \theta_{0}, z-z_{0}\right) d\left(z-z_{0}\right) \\
& q^{\prime}\left(\theta, \theta_{0}\right)=\int_{-\infty}^{\infty} e^{i k_{z}\left(z-z_{0}\right)} \frac{\partial Q}{\partial n}\left(\theta, \theta_{0}, z-z_{0}\right) d\left(z-z_{0}\right)
\end{aligned}
$$

Note in the above that $g_{k}, t_{k i}, q$ and $q^{\prime}$ are given functions, and $U_{i}$ or $U_{k}$ and $P$ are unknown functions. Also note that index $i$ corresponds to $\theta$ and $k$ corresponds to $\theta_{0}$.

It can be shown that an integral of a product of two functions may be approximated by the sum of the products of (i) the value of the second function at $N$ sampling points and (ii) a weight coefficient depending on the first function over the integration interval such that

$$
\begin{aligned}
& \int_{a}^{b} f_{1}(x) f_{2}(x) d x=\sum_{n=1}^{N} \alpha_{n} f_{2}\left(x_{n}\right) \\
& \alpha_{n}=\alpha_{n}\left[f_{1}(x), n, a, b\right]
\end{aligned}
$$

The boundary is now discretized by $N$ sampling points. It should be noted that for each sampling point, four unknowns are involved, namely, $U_{i}, i=1,2,3$ and $P$. As a result, there are totally $4 N$ unknowns. We shall then replace $U_{k}\left(\theta_{m}\right), k=1,2,3, m=1,2, \ldots . N$ by $U_{\infty}, \alpha=1,2,3 \ldots .3 N$; $U_{i}\left(\theta_{l}\right), i=1,2,3, l=1,2, \ldots N$ by $U_{\beta}, \beta=1,2,3 \ldots . .3 N ; P\left(\theta_{l}\right)$ by $P_{l} \quad l=1,2,3 \ldots . . N ; P\left(\theta_{m}\right)$ by $P_{m}, P_{0}\left(\theta_{m}\right)$ by $P_{m}^{0}$, 
$m=1,2,3 \ldots . N$. In view of quadrature rule (28) and the above notation conventions, Eqs. (25-26) can be represented by

$$
\begin{gathered}
C U_{\alpha}=-\sum_{l=1}^{N} g_{\alpha l} P_{l}-\sum_{\beta=1}^{3 N} t_{\alpha \beta} U_{\beta} \\
C_{b} P_{m}=\sum_{\beta=1}^{3 N} q_{m l} U_{\beta} n_{l \beta}-\sum_{l=1}^{N} q_{m l}^{\prime} P_{l}
\end{gathered}
$$

or

$$
\begin{gathered}
\sum_{\beta=1}^{3 N}\left(C \delta_{\alpha \beta}+t_{\alpha \beta}\right) U_{\beta}+\sum_{l=0}^{N} g_{\alpha l} P_{l}=0 \\
-\sum_{l=1}^{3 N} q_{m l} n_{l \beta} U_{\beta}+\sum_{l=1}^{N}\left(C_{b} \delta_{m l}+q_{m l}^{\prime}\right) P_{l}=p^{0}{ }_{m}
\end{gathered}
$$

where $g_{\alpha l}, t_{\alpha \beta}, q_{m l}$ and $q_{m l}^{\prime}$ are weight coefficients corresponding to given functions $g_{k}, t_{k i}, q$ and $q^{\prime}$, respectively. It should be pointed out in Equation (30) that quantities with two indices represent matrices and are denoted by the same letters of boldface. Similarly, quantities with one index represent vectors and are denoted by the same letters of boldface. Thus Equation (30) can be written in the matrix form below.

$$
\left[\begin{array}{cc}
c I_{1}+t & g \\
-q & C_{b} I_{2}+q
\end{array}\right]\left\{\begin{array}{l}
U \\
P
\end{array}\right\}=\left\{\begin{array}{l}
0 \\
P_{0}
\end{array}\right\}
$$

where matrices $I_{1}, t$ have a dimension of $3 N \times 3 N, I_{2}, q^{\prime}$ of $N \times N, q$ of $3 N \times N$, and $g$ of $N \times 3 N$. The dimension of vector $\boldsymbol{P}$ and $\boldsymbol{P}^{0}$ is $N$ and that of $\boldsymbol{U}$ is $3 N$.

\section{The $\theta$-Integrals}

The $\theta$-Integrals are those in Eqs. (25-26). Equation (28) gives a general rule of the quadrature. For instance, a standard Gaussian quadrature can used.

\section{Solution of ITBIE}

In the numerical solution of the ITBIEs, Eqs. (25-26), the circular boundary is discretized by nodes. Each node has four unknowns: $U_{r}, U_{\theta}, U_{z}$ and $P$. These nodal unknowns form a vector $\{U\}$ with dimension $4 N \times 1$ where $N$ is the total number of nodes. The free term on the right hand side of Equation (26) at the nodes forms a given vector $\left\{U_{0}\right\}$. The application of a quadrature rule to the BIEs then leads to a linear system of equations ${ }^{[5]}$ : 


$$
[M]\{U\}=\left\{U_{0}\right\}
$$

where matrix $[M]$ is obtained from the integral transformations of fundamental solutions, $G_{i k}(\xi, x)$, $T_{i j}^{k}(\xi, x), Q(\xi, x)$ and $\partial Q(\xi, x) / \partial r$.

\section{The $k_{z}$-Integrals}

The above steps have lead to the solution of $\left[U_{r}, U_{\theta}, U_{z}, P\right]^{T}$ for a given vertical, $k_{z}$. In order to obtain the full frequency response, $\left[u_{*} u_{{ }_{\theta}} u{ }_{z} p\right]^{T}$, wavenumber integral Equation (8) must be evaluated over an adequate number of sampling points of $k_{z}$. This again uses the adaptive $\mathrm{MCC}$ integration technique.

\section{Summary of Solution Procedure}

as follows.

The proposed solution procedure of the ITBIE approach can be summarized

(i) Discretize the boundary, $r=a$, by uniformly distributed nodes whose number must increase with frequency;

(ii) Obtain $G_{i k}(\xi, x), T_{i j}{ }^{k}(\xi, x), Q(\xi, x)$ and $\partial Q(\xi, x) / \partial r$ of equation (13) for all SR pairs, leaving $z=\xi_{3}-x_{3}$ as a variable;

(iii) Evaluate the $z$-integrals defined in equation (14) over all integral transformed SR pairs and store the fitting information;

(iv) Use result from step (iii) and equation (28) to obtain a submatrix similar to equation (31) for each node;

(v) Assemble matrix $[M]$ from all submatrices to obtain the global matrix $[M]$ defined in equation (32);

(vi) Solve equation (32) for $\{U\}$ on the boundary;

(vii) Use equation (18) and the result of (vi) to evaluate the reflected part of the pressure in the $k_{z}$ domain, $P$ - $P^{\circ}$, at specified detectors;

(viii) Carry out adaptive integration in the $k_{z}$ domain, equation (16), using the fitting information stored in step (iii) and obtain the reflected part of the pressure at detectors in the frequency domain, $p-p^{\circ}$; 
(ix) Add the source term, $p^{\circ}$, given by the first equation of (8) to the reflected part of the pressure to obtain the total pressure, $p$.

\section{Computational Aspects}

There are two major issues in the numerical aspects of the ITBIE approach. The first issue is the computer time. It should be mentioned that because of the integral transform, the DOF of the final linear system of equations in this approach is quite small $(<100)$. Therefore the most time-consuming part in the computation is the evaluation of the coefficients of the matrix in equation (31) which involve double infinite integrals defined by Eqs. (10) and (19). In view of this, a number of steps have been taken to minimize the computer time while maintaining sufficient accuracy.

(i) The $k_{\rho}$-integrals in equation (10), which arise in the Green's functions and associated stresses, are evaluated using the MCC adaptive integration method. It is noted that one half of the node pairs shares the same $\zeta$ separation with the other half. Therefore, only one half of the node pairs need the curve fitting of the kernel. Since curve fitting takes $80-90 \%$ of the total time of MCC integration, this additional saving is significant.

(ii) The $z$-integrals in equation (19) whose kernel is the Green's functions and associated stresses, are also evaluated by the MCC adaptive integration method. For different values of vertical wavenumber, $k_{z}$, the results of these $z$-integrals are different. Fortunately, $k_{z}$ appears in the factor $\exp \left(i_{z} z\right)$ only and is absent in the kernel. Therefore, the curve fitting remains unchanged for any $k_{z}$. There are in general hundreds of $k_{z}$ used in a $k_{z}$-integration. This saving can be seen to be much greater than that in the $k_{\rho}$-integration. However, the fitting information of the $z$-integrals for all SR pairs of the mesh must be documented before the solution of ITBIE, which requires a significant amount of RAM spaces (about 30 megabytes for a six-element mesh).

(iii) The setup of the coefficient matrix [M] of equation (32) can take advantage of the symmetry of the geometry and the loading. It can be shown that the unknown field variables on the boundary have symmetry about both $x-z$ and $y-z$ planes. Then the original $N \mathrm{x} N$ matrix can be decomposed into two $(N / 2) \times(N / 2)$ matrices, one corresponding to symmetric loading and the other antisymmetric. In each case, only unknowns at one quarter of the nodes are independent. As a result, the total computational effort is reduced to its $50 \%$. The relationship between the original matrix and decomposed matrices is given in Appendix C.

(iv) For the $\theta$-integration in equations (25-26), a 4-point Gaussian quadrature is employed. In an effort to enhance sampling ability without increasing the number of unknowns, we use the quadratic fitting instead of linear fitting, thus yielding $N_{s}=8 / 2=4$.

(v) The $k_{z}$-integration in equation (16) is again performed by using the MCC adaptive integration method. As mentioned earlier, all the calculations for the $k_{\rho}$-integrals and $z$-integrals have been carried out in step (ii). As a result, this step involves mainly bookkeeping. 
(vi) The final target of the calculation is the pressure at specified detectors. After solving ITBIE equations (25-26) for unknown displacements and pressure on boundary, and using equations (25-26) again for the field points, the total pressure at these points in the $k_{z}$ domain can be obtained. In the actual calculation, it is more convenient to obtain the reflected part of the pressure by subtracting the incident part, $P^{\circ}$, in the $k_{z}$ domain. The total pressure can be obtained by adding the incident part back in the frequency domain. Note that the latter is given in closed form (Equation 8). Thus numerical difficulties in the calculation of the source term in the $k_{z}$ domain associated with the singularity at the center of the borehole are avoided.

The second issue is the RAM space. As discussed above, the fitting information of the $z$-integration must be stored for the efficient evaluation of $k_{z}$-integrals. If the available RAM space is insufficient, hard disk storage can be a substitute. This however will increase the computer time by a factor of 10 to 20 . An effort is being made to reduce the amount of information to be stored.

\section{Tests}

include:

Various tests have been done in many stages of the computer program. These

(1) Tests of the $k_{\rho}$-integration were conducted using integrals with known results given in Appendix D;

(2) Tests of the Green's functions for the TI medium were conducted using the Green's functions for the isotropic medium given in Section 4;

(3) Tests of the $z$-integration were done using integrals with known results given in Appendix D;

(4) Tests of the $\theta$-integration were done using integrals with known results given in Appendix D;

(5) Tests of the $k_{z}$-integration were done using integrals with known results given in Appendix D;

(6) Tests of the $k_{\rho}$-domain result were done using the "exact" solution for isotropic case which is obtained through a standard method ${ }^{[7]}$.

\section{Future Improvements of Efficiency through Approximations}

\section{(1) Far-field Approximation of the Green's functions}

The $z$-integration uses the Green's functions and associated stress functions for $z$ ranging from 0 to infinity. It is therefore desired their far-field expressions be obtained to reduce computational efforts, especially when the medium is TI for which these functions are in the form of $k_{\rho}$-integrals. Since the $z$-axis coincides with the direction of $\rho$ when $\psi=90^{\circ}$, we need far-field approximations for large $k_{\rho}^{(\mathrm{P})} \rho$ and $k_{\rho}^{(\mathrm{S})} \rho$, where $k_{\rho}^{(\mathrm{P})}$ and $k_{\rho}^{(\mathrm{S})}$ are the quasi-P and quasi-S 
wavenumbers in $\rho$ direction. A literature survey is being conducted in order to find work done in this area.

(2) Asymptotic expression for the integration of infinite tail of the $z$-integrals based on the far field expressions of the Green's functions when they are available.

\section{REFERENCES}

[1] P.-C. Xu and A. K. Mal, "An Adaptive Integration Scheme for Irregularly Oscillatory Functions", Wave Motion, Vol. 7, 235-243, 1985.

[2] P.-C. Xu and A. K. Mal, Calculation of the Inplane Green's Functions for Layered Solids, Bull. Seism. Soc. Am., 77(4), 1823-1837, 1987.

[3] J. O. Parra, V. R. Sturdivant, and P.-C. Xu, "Interwell Seismic Transmission and Reflection Through a Dipping Low-Velocity Layer", J. Acoust. Soc. Am. 93(4), 19541968, 1993.

[4] P. P. Goswami, T. J. Rudolphi, F.J. Rizzo, and D.J. Shippy, "A Boundary Element Model for Acoustic-Elastic Interaction with Applications in Ultrasonic NDE", $J$. Nondestr. Eval., 9(2/3), 101-112, 1990.

[5] J. O. Parra, "Analysis of Seismic Wave Propagation in Stratified Transversely Isotropic Media, Technical Report, SwrI, 1991.

[6] P.-C. Xu, " Boundary Integral Equation Method for P-SV Wave Interaction with Depositional Anomalies in an Unbounded Medium", Technical Report to SwRI, September 1995.

[7] A. L. Kurkjian and S.-K. Chang, "Acoustic Multipole Sources in Fluid-Filled Boreholes", Geophysics, 51(1), 148-163, 1986. 


\section{APPENDIX A}

EXPRESSIONS OF THE P-SV AND SH PARTS OF GREEN'S FUNCTIONS FOR TI MEDIA

$$
\begin{aligned}
& Z_{1}=C_{o}\left(C_{13}+C_{44}\right) k_{p}\left[-e^{-v|\zeta|}+e^{-x|\zeta|}\right] \\
& Z_{2}=C_{o} C_{44}\left[\frac{v^{2}-\beta^{2}}{v} e^{-v|\zeta|}-\frac{x^{2}-\beta^{2}}{x} e^{-x|\zeta|}\right] \\
& X_{1}=C_{o} C_{33}\left[\frac{v^{2}-\alpha^{2}}{v} e^{-v|\zeta|}-\frac{x^{2}-\alpha^{2}}{x} e^{-x|\zeta|}\right] \\
& Y=-\frac{1}{4 \pi v_{3} C_{44}} e^{-s|\zeta|}
\end{aligned}
$$

where $v, \chi, s$ are wavenumbers in $\zeta$-direction for quasi $\mathbf{p}, \mathbf{S V}$ and SH waves. Their definition can be found in Reference $C 7$. 


$$
\begin{aligned}
& I_{1}=\int_{0}^{\infty} Z_{1}\left(k_{\rho}, \zeta\right) k_{\rho} J_{0}\left(k_{\rho} \rho\right) d k_{\rho} \\
& \mathrm{I}_{2}=\int_{0}^{\infty} \mathrm{Z}_{1}\left(\mathrm{k}_{\rho}, \zeta\right) \mathrm{k}_{\rho} \mathrm{J}_{2}\left(\mathrm{k}_{\rho} \rho\right) d \mathrm{k}_{\rho} \\
& I_{3}=\int_{0}^{\infty} \frac{\partial z_{1}}{\partial \zeta}\left(k_{\rho}, \zeta\right) J_{1}\left(k_{\rho} \rho\right) d k_{\rho} \\
& I_{4}=\int_{0}^{\infty} Z_{2}\left(k_{\rho}, \zeta\right) J_{0}\left(k_{\rho} \rho\right) d k_{\rho} \\
& I_{5}=\int_{0} Z_{2}\left(k_{\rho}, \zeta\right) k_{\rho} J_{1}\left(k_{\rho} \rho\right) d k_{\rho} \\
& I_{6}=\int_{0}^{\infty} \frac{\partial Z_{2}}{\partial \zeta}\left(k_{\rho}, \zeta\right) J_{0}\left(k_{\rho}, \rho\right) d k_{\rho} \\
& I_{7}=\int_{0}^{\infty} X_{1}\left(k_{\rho}, \zeta\right) J_{0}\left(k_{\rho} \rho\right) d k_{\rho} \\
& \mathrm{I}_{8}=\int_{0}^{\infty} \mathrm{X}_{1}\left(\mathrm{k}_{\rho}, \zeta\right) \mathrm{J}_{2}\left(\mathrm{k}_{\rho} \rho\right) \mathrm{dk} \mathrm{k}_{\rho} \\
& \mathrm{I}_{9}=\int_{0}^{\infty} \mathrm{X}_{1}\left(\mathrm{k}_{\rho}, \zeta\right) \mathrm{k}_{\rho} \mathrm{J}_{1}\left(\mathrm{k}_{\rho} \rho\right) \mathrm{dk_{ \rho }} \\
& I_{10}=\int_{0}^{\infty} \frac{\partial X_{1}}{\partial \zeta}\left(k_{\rho}, \zeta\right) J_{0}\left(k_{\rho} \rho\right) d k_{\rho} \\
& I_{11}=\int_{0}^{\infty} \frac{\partial X_{1}}{\partial \zeta}\left(k_{\rho}, \zeta\right) J_{2}\left(k_{\rho} \rho\right) d k_{\rho} \\
& I_{12}=\int_{0}^{\infty} X_{2}\left(k_{\rho}, \zeta\right) k_{\rho} J_{0}\left(k_{\rho} \rho\right) d k_{\rho} \\
& I_{13}=\int_{0}^{\infty} X_{2}\left(k_{\rho}, \zeta\right) k_{\rho} J_{2}\left(k_{\rho} \rho\right) d k_{\rho}
\end{aligned}
$$




$$
\begin{aligned}
& I_{14}=\int_{0}^{\infty} \frac{\partial X_{2}}{\partial \zeta}\left(k_{\rho}, \zeta\right) J_{1}\left(k_{\rho} \rho\right) d k_{\rho} \\
& I_{15}=\int_{0}^{\infty} Y\left(k_{\rho}, \zeta\right) k_{\rho} J_{1}\left(k_{\rho} \rho\right) d k_{\rho} \\
& \left.I_{16}=\int_{0}^{\infty} Y\left(k_{\rho}, \zeta\right) J_{0}\left(k_{\rho} \rho\right) d k_{\rho}\right) \\
& I_{17}=\int_{0}^{\infty} Y\left(k_{\rho}, \zeta\right) J_{2}\left(k_{\rho} \rho\right) d k_{\rho} \\
& I_{18}=\int_{0}^{\infty} \frac{\partial Y}{\partial \zeta}\left(k_{\rho}, \zeta\right) J_{0}\left(k_{\rho} \rho\right) d k_{\rho} \\
& I_{19}=\int_{0}^{\infty} \frac{\partial Y}{\partial \zeta}\left(k_{\rho}, \zeta\right) J_{2}\left(k_{\rho} \rho\right) d k_{\rho}
\end{aligned}
$$




\section{APPENDIX C SYMMETRIC AND ANTISYMMETRIC DECOMPOSITION OF THE} COEFFICIENT MATRIX

Consider an 8-node mesh. Assuming each node has only one unknown: $U_{1}, U_{2},--U_{3}$. Because of the symmetrics with respect to $\mathrm{x}$-axis and $\mathrm{y}$ axis, the only independent unknowns are $\mathrm{U}_{1}$, $\mathrm{U}_{2}, \mathrm{U}_{3}$. In fact,

$$
\begin{aligned}
& U_{4}= \pm U_{2} \\
& U_{5}= \pm U_{1} \\
& U_{6}= \pm U_{2} \\
& U_{7}= \pm U_{3} \\
& U_{8}= \pm U_{2}
\end{aligned}
$$

original equation:

$$
\begin{aligned}
& {[M]\{U\}} \\
& 8 x 88 \times 1
\end{aligned}=\begin{gathered}
p^{0} \\
8 \times 1
\end{gathered}
$$

New equations:

$$
\left\{\begin{array}{c}
{[M]^{5}\left\{u^{(5)}\right\}=\left\{p^{0(5)}\right\}} \\
3 \times 3 \text { 3x1 } \\
3 \times 1 \\
{\left[M^{(A)}\right]\left\{u^{(A)}\right\}=\left\{p^{O(A)}\right\}} \\
3 \times 3 \quad 3 \times 1
\end{array}\right.
$$

where 


$$
\begin{aligned}
& M_{11}^{(\alpha)}=m_{11}+f_{1} m_{15} \\
& M_{12}^{(\alpha)}=m_{12}+f_{2} m_{14}+f_{3} m_{16}+f_{4} m_{18} \\
& M_{13}^{(\alpha)}=m_{13}+f_{5} m_{17} \\
& M_{21}^{(\alpha)}=m_{21}+f_{6} m_{25} \\
& M_{22}^{(\alpha)}=m_{22}+f_{7} m_{24}+f_{8} m_{26}+f_{9} m_{28} \\
& M_{23}^{(\alpha)}=m_{23}+f_{10} m_{27} \\
& M_{31}^{\alpha}=m_{31}+f_{11} m_{35} \\
& M_{32}^{\alpha}=m_{32}+f_{12} m_{34}+f_{13} m_{36}+f_{14} m_{38} \\
& M_{33}^{\alpha}=m_{33}+f_{15} m_{37} \\
& \mathrm{~A}=5 \text { or } \mathrm{A}
\end{aligned}
$$

$S$ denotes symmetric loading and $A$ antisymmetric loading. $f_{i}= \pm 1, i=1,2, \ldots 15$. The sign depends on the particular loading.

$f_{i}= \pm 1, i=1,2, \ldots 15$. The sign depends on the particular loading. 
1. $\mathrm{k} \rho$ - integrals

$$
\int_{0}^{\infty} \frac{k_{\rho} e-v z}{v} J_{0}\left(k_{\rho} \rho\right) d k \rho=\frac{e^{i k R}}{R}
$$

where

$$
\begin{aligned}
& R=\sqrt{\rho^{2}+z^{2}}, \quad \mathrm{v}=\sqrt{k_{\rho}^{2}-k^{2}} \quad k_{\rho}>k \\
& =i \sqrt{k^{2}-k_{\rho}^{2}} \quad k_{\rho}<k \\
& \int_{0}^{\infty} e^{-k_{p} z} J_{1}\left(k_{\rho} \rho\right) d k_{\rho}=\frac{R-Z}{\rho R} \\
& \int_{0}^{\infty} k_{\rho} e^{-k_{\rho} z} J_{0}\left(k_{\rho} \rho\right) d k_{\rho}=\frac{Z}{R^{3}} \\
& \int_{0}^{\infty} k_{\rho} e^{-k_{\rho} z} J_{1}\left(k_{\rho} \rho\right) d k_{\rho}=\frac{\rho}{R^{3}} \\
& \int_{0}^{\infty} k_{\rho} e^{-k_{\rho} z} J_{2}\left(k_{\rho} \rho\right) d k_{\rho}=\frac{2(R-Z)}{\rho^{2} R}-\frac{Z}{R^{3}}
\end{aligned}
$$

5. 0 - integrals

$$
\int_{0}^{2 \pi} e^{i z \cos ^{\frac{0}{2}}} \alpha \theta=2 \pi J_{0}(Z)
$$




$$
\begin{gathered}
\int_{0}^{2 \pi} e^{i z \cos \frac{\theta}{2}} \cos \frac{\theta}{2} d \theta=2 \pi i J_{1}(Z) \\
\int_{0}^{2 \pi} H_{0}\left(2 Z \sin \left|\frac{\theta}{2}\right|\right) d \theta=2 \pi J_{0}(Z) H_{0}(Z) \\
\int_{0}^{2 \pi} H_{0}\left(2 Z \sin \left|\frac{\theta}{2}\right|\right) \cos \theta d \theta=2 \pi J_{1}(Z) H_{1}(Z) \\
\int_{0}^{2 \pi} H_{0}\left(2 Z \sin \left|\frac{\theta}{2}\right|\right) \sin \frac{\theta}{2} d \theta=\pi\left[J_{0}(Z) H_{0}(Z)+J_{1}(Z) H_{0}(Z)\right]
\end{gathered}
$$

3. $\quad \mathrm{Z}$ - integrals and $\mathrm{k}_{2}$ - integrals

$$
\begin{gathered}
\int_{-\infty}^{\infty} \frac{e^{i k z}}{z^{2}+a^{2}} d z=\frac{\pi}{a} e^{-k a} \\
\int_{-\infty}^{\infty} \frac{z e^{i k z}}{z^{2}+a^{2}} d z=\pi i e^{-k a} \\
\int_{-\alpha}^{\alpha} e^{i b z} e^{i a z} d Z=\frac{2}{a+b} \sin \alpha(a+b) \\
\int_{-\infty}^{\infty} e^{i a t} H_{0}\left(b \sqrt{d^{2}-z^{2}}\right) d z=-2 i \frac{e^{i d \sqrt{b^{2}+a^{2}}}}{\sqrt{b^{2}+a^{2}}} \\
\int_{-\infty}^{\infty} \frac{e^{i p \sqrt{a^{2}+z^{2}} e^{i k z}}}{\sqrt{z^{2} e a^{2}}} d z=i \pi H_{0}(a p)
\end{gathered}
$$




\section{SEMIANALYTICAL APPROACH TO MULTIPHASE FLOW CALCULATIONS}

\section{A. Multiphase Streamline Modeling in Three-Dimensions}

Recently three dimensional streamline modeling of multiphase flow has gained increasing popularity ${ }^{14}$. It has also become a very important tool as a fast and reliable flow simulator for solving inverse problems in reservoir characterization ${ }^{5}$. Such streamline simulation decouples multidimensional problems into a series of one-dimensional problems which can then be solved analytically ${ }^{6}$ or numerically. ${ }^{7-11}$ Significant speed over traditional finite difference models has been shown ${ }^{4}$. However, such comparisons have been largely limited to simple single pattern configurations, e.g. 5-spots or line drives ${ }^{12}$. Field scale application of three dimensional streamline modeling has been very limited ${ }^{2}$. The primary limitations with regard to field applications have been changing well configurations due to infill drilling, zone isolations, recompletions, etc. A critical issue here is the remapping of streamlines and hence fluid saturations as the dynamics of field conditions dictate.

Past efforts to handle infill drilling during streamline simulation have been to average $^{13}$ streamlines over an underlying grid and then to proceed with numerical computations along streamlines. However, it is well known that such averaging of streamlines leads to numerical dispersion. Thus, such an approach undermines one of the major strengths of streamline modeling, which in addition to the fast solution, is to preserve the self-sharpening nature of the saturation fronts during waterflooding. Averaging of streamlines in conjunction with lower order numerical solution of multiphase flow equations along streamlines leads to a significant loss in accuracy.

We present two major improvements to the existing streamline modeling. First, instead of averaging streamlines during changing well conditions, we have used a 3-D mapping algorithm ${ }^{14-15}$ for streamlines. The algorithm uses a trivariate function to remap streamlines and saturations during infill driling using a modified quadratic shepard method. The 3-D mapping algorithm is robust and does not lead to smearing of saturation fronts. Second, along streamlines we have used a third-order total variation diminishing (TVD) ${ }^{7-9}$ scheme to solve the multiphase flow problem to prevent numerical truncation error. ${ }^{10-11}$ Comparison with the existing method shows significant improvement in accuracy without any loss in computational efficiency. We illustrate our approach using synthetic as well as field applications and comparing the results of streamline simulation with numerical simulation. We compare different numerical schemes for saturation solution along streamlines with the analytical solution using a synthetic example of a $2 \mathrm{D}$, homogeneous quarter 5 spot pattern under uniform initial saturation conditions. The extension to non-uniform initial conditions is validated using a numerical simulator for a synthetic example involving a 5-spot to 9spot pattern conversion. The approach is then applied to an example with multiwell configuration. Finally, a field application is presented. The field case involves waterflood predictions in a highly heterogeneous fractured carbonate reservoir -- North Robertson field in West Texas. Production performance from multiple patterns (simulation grid of $50 \times 25 \times 12$ ) consisting of 27 producers and 15 injectors have been simulated using the streamline model to demonstrate the power and versatility of the approach. 
The streamline approach for modeling multiphase flow in reservoirs relies on a unique transit time algorithm presented by Datta-Gupta and King and Peddibhotla et al $^{2}$. The application of streamline approach in three dimensions is discussed briefly in this section.

Streamline Generation: For incompressible flow in a non-deformable permeable media, the velocity will be driven by a pressure field and the total mobility is a function of position.

$$
\nabla .(\lambda, \nabla p)=q \delta_{i j}
$$

The pressure field is generated using a finite difference scheme that yields a symmetric and positive-definite system of equations which lend themselves to efficient solvers such as Cholesky decomposition or preconditioned conjugate gradient techniques. Once the pressure field is generated, the face velocities for grid blocks are obtained using Darcy's law. The velocity field within grid blocks is derived using the simplification that in the finite element representation of the standard lowest order finite difference scheme, each velocity varies linearly through a grid block and each velocity depends only on its own coordinate. Thus, the velocities at each of the six gridblock faces (x1 or west, $\mathrm{x} 2$ or east, y1 or south, y2 or north, $\mathrm{z} 1$ or top, $\mathrm{z} 2$ or bottom) are calculated as follows:

$$
\begin{aligned}
& v_{x}=a_{x}\left(x-x_{1}\right)+v_{x 1} \\
& v_{y}=a_{y}\left(y-y_{1}\right)+v_{y 1} \\
& v_{z}=a_{z}\left(z-z_{1}\right)+v_{z 1}
\end{aligned}
$$

For example, as shown in Fig.2, $v_{x 1}$ and $v_{x 2}$ are the $x$-velocities on the west and east faces of a gridblock. In this example gridblock, the streamline shown is entering through the west face and exiting through the north face. In the above equation, the slope of velocity, $a$, in each direction within a gridblock is

$$
\begin{aligned}
& a_{x}=\left(v_{x 2}-v_{x 1}\right) / \Delta x \\
& a_{y}=\left(v_{y 2}-v_{y 1}\right) / \Delta y \\
& a_{z}=\left(v_{z 2}-v_{z 1}\right) / \Delta z
\end{aligned}
$$

The particle travel time, $\tau$, within the gridblock can now be computed by direct integration as follows:

$$
d \tau=\frac{d t}{\phi}=\frac{d x}{v_{x}}=\frac{d y}{v_{y}}=\frac{d z}{v_{z}}
$$

The transit time of a tracer particle from an arbitrary location $\left(\mathrm{x}_{0}, \mathrm{y}_{0}, \mathrm{z}_{0}\right)$ within the gridblock to a face can thus be calculated using the slope of velocity in the direction of that face. For example in the x-direction: 


$$
\begin{aligned}
& \Delta t_{x i}=\left(1 / a_{x}\right) \ln \left(\frac{v_{x i}}{v_{x o}}\right), \quad i=1,2 \\
& \Delta t_{y i}=\left(1 / a_{y}\right) \ln \left(\frac{v_{y i}}{v_{y o}}\right), \quad i=1,2 \\
& \Delta t_{z i}=\left(1 / a_{z}\right) \ln \left(\frac{v_{z i}}{v_{z o}}\right), \quad i=1,2
\end{aligned}
$$

where $\mathrm{i}=1,2$ are the gridblock faces in each direction. Since the particle must exit through one of the faces, the actual transit time of the tracer particle will be given by the minimum $\Delta t$ over allowed edges.

$$
\Delta t_{x i}=\operatorname{MIN}\left(\Delta t_{x 1}, \Delta t_{x 2}, \Delta t_{y 1}, \Delta t_{y 2}, \Delta t_{z 1}, \Delta t_{z 2}\right)
$$

Once the particle travel time across the grid block is computed, its exit coordinates from the gridblock can then be calculated. Exit coordinates are specified by the $\mathrm{x}, \mathrm{y}$ and $\mathrm{z}$ location.

$$
\begin{aligned}
& x=x_{1}+\left(1 / a_{x}\right)\left[v_{x o} e^{a_{x} \Delta t}-v_{x 1}\right] \\
& y=y_{1}+\left(1 / a_{y}\right)\left[v_{y o} e^{a_{y} \Delta t}-v_{y 1}\right] \\
& z=z_{1}+\left(1 / a_{z}\right)\left[v_{z o} e^{a_{z} \Delta t}-v_{z 1}\right]
\end{aligned}
$$

The particle travel time, $\tau$, to a producer or any location in the flow domain can be obtained by starting the trajectory at the point of interest and following the streamline backwards in time until an injector is reached. The underlying assumption is that the streamlines do not shift significantly with time. Particularly for waterflood applications this is expected to be a good approximation since the total mobility is almost always stable.

One-dimensional Solution Along Streamlines: The Buckley-Leverett ${ }^{6}$ equation describing immiscible two-phase displacement can be written in terms of the travel time coordinates as follows:

$$
\frac{\partial S_{w}}{\partial t}+\frac{\partial F\left(S_{w}\right)}{\partial \tau}=0
$$

where $S_{w}$ is the water saturation, $t$ is actual time or simulation time, and $F\left(S_{w}\right)$ or $f_{w}$ is the fractional flow of water given by 


$$
f_{w}=\frac{\lambda_{w}}{\left[\lambda_{w}+\lambda_{o}\right]}
$$

In the above equation, $\lambda_{w}$ and $\lambda_{0}$ are phase mobilities which are functions of relative permeabilities, $\mathrm{k}_{\mathrm{r}}$, and viscosities, $\mu$.

$$
\begin{aligned}
& \lambda_{o}=\frac{k k_{r o}}{\mu_{o}} \\
& \lambda_{w}=\frac{k k_{r w}}{\mu_{w}}
\end{aligned}
$$

and

$$
\begin{aligned}
& k_{r o}=k_{r o n} *\left[\frac{1-S_{w}-S_{o r}}{1-S_{w c}-S_{o r}}\right]^{n_{o}} \\
& k_{r w}=k_{r w n} *\left[\frac{S_{w}-S_{w c}}{1 .-S_{w c}-S_{o r}}\right]^{n_{w}}
\end{aligned}
$$

From Eq. 8 along a streamline the solution for water saturation distribution, $S_{w}(x, y, z, t)$, will be given by:

$$
\frac{\tau(x, y, z, t)}{t}=\frac{d f_{w}}{d S_{w}}
$$

Note that in the above equation, the right hand side corresponds to the slope of the fractional flow curve and thus, is a single-valued function of water saturation. Finally, the oil rate of a producer, $\mathrm{q}_{\mathrm{o}}$, will be given by:

$$
q_{o}=q_{t}\left[\frac{\sum_{l=1}^{N_{s l}}\left[1-\left(f_{w}(x, y, z, t)\right)_{l}\right]}{N_{s l}}\right]
$$

where $q_{t}$ is the total specified production rate of the well, and $\mathrm{N}_{\mathrm{st}}$ is the number of streamlines converging to that producer. The individual well production history can be obtained by integrating over all the streamlines arriving at a given producer.

The analytical solution given by Eq. 12 holds good only under uniform initial conditions. However, typical waterflood involves drilling of infill injectors and producers over the life of the 
project. Clearly, the uniform initial conditions assumptions no longer holds good under such conditions and the 1D solution along each streamline is then obtained numerically. Eq.8 is discretized as follows:

$$
\frac{S_{w}{ }^{n+1}-S_{w}{ }^{n}}{\Delta t}+\frac{F_{i+1 / 2}-F_{i-1 / 2}}{\Delta \tau}=0
$$

As usual, $\mathrm{F}_{\mathrm{i}+1 / 2}$ is at the boundary between gridblock $\mathrm{i}$ and $\mathrm{i}+1$, and $\mathrm{n}$ is the timestep counter. Fig. 3 is an illustration of descretization of streamline in space. A high resolution technique called Total Variation Diminishing (TVD) ${ }^{7-9}$ is used for the numerical solution to minimize artifacts such as numerical dispersion.

The total variation is defined as follows:

$$
T V\left(F^{n+1}\right)=\sum_{i=1}^{N 1 D-1}\left|F_{i+1}^{n+1}-F_{i}^{n+1}\right|
$$

where NID is the number of gridblocks overlain on each streamline. To ensure monotonicity and to avoid spurious oscillations, we must satisfy the total variation diminishing criteria as suggested by Harten $^{8}$ :

$$
T V\left(F_{i}^{n+1}\right) \leq T V\left(F_{i}^{n}\right)
$$

As discussed by Sweby ${ }^{11}$, the flux term at the block interface is approximated as a first order flux term and an anti-diffusive corrective term:

$$
F_{i+1 / 2}=\left(f_{w}\right)_{i}^{n}+\varphi(r)\left[\frac{\left(f_{w}\right)_{i+1}{ }^{n}-\left(f_{w}\right)_{i}{ }^{n}}{2}\right]
$$

where $\varphi(r)$, the flux limiter, governs the order of discretization as follows:

$$
\begin{array}{rlrl}
\varphi(r) & =r & & , 2 \text { pt.upstream weighting } \\
& =1 \quad, \text { midpt.weighting } \\
& =\frac{2}{3}+\frac{1}{3} r, \text { weighted average }
\end{array}
$$

The measure of smoothness of the data, $r$, is determined as

$$
r=\left(\frac{\left(f_{w}\right)_{i}^{n}-\left(f_{w}\right)_{i-1}^{n}}{\left(f_{w}\right)_{i+1}^{n}-\left(f_{w}\right)_{i}^{n}}\right) *\left(\frac{\tau_{i+1}-\tau_{i}}{\tau_{i}-\tau_{i-1}}\right)
$$


Using a upstream weighted numerical formulation results in a stable but dispersed solution. Midpoint weighting results in a more accurate solution, but causes instability. The optimum choice lies somewhere in between, i.e. a weighted average as recommended by Leonard ${ }^{10}$ and . Fig. 4 is an comparison of the different mathematical formulations.

The limiting function, $\varphi(r)$, is chosen such that the limited anti-diffusive term is maximized in amplitude subject to the constraint that the resulting mathematical formulation is a Total Variation Diminishing (TVD) formulation. Sweby ${ }^{11}$ derived the algebraic conditions on the limiting function that guarantee this property.

$0 \leq \frac{\varphi(r)}{r}, \quad \varphi(r) \leq 2$

Eq.25 results in the following TVD limiters for the higher order mathematical formulations described above in Eq.23,

$$
\begin{array}{ll}
\varphi(r)=\operatorname{Max}(0, \operatorname{Min}(r, 2)) & 2 p t . \text { upstreamweighting } \\
\varphi(r)=\operatorname{Max}(0, \operatorname{Min}(r, 2)), & \text { Midpt.weighting } \\
\varphi(r)=\operatorname{Max}\left(0, \operatorname{Min}\left(2,2 r, \varphi^{L E}\right)\right) & \text { Third order Leonard }
\end{array}
$$

Finally, the water saturation is then determined as

$$
S_{w}{ }^{n+1}=S_{w}{ }^{n}-\frac{\Delta t}{\Delta \tau} *\left(F_{i+1 / 2}-F_{i-1 / 2}\right)
$$

where $\Delta \mathrm{t}$ is the timestep for numerical solution.

Mapping Streamlines to Streamlines at Infill: At infill time, the intersection of streamlines with gridblock faces, and the water saturation and travel time at these locations are known. With the change in well configuration, the streamlines are redefined in space to account for the new wells. As a result, the intersection points of streamlines with gridblock faces also change. The water saturation at these new intersection points are obtained using 3D interpolation from the original intersection-point water saturations. Renka ${ }^{14,15}$ presented the $3 \mathrm{D}$ interpolation algorithm, QSHEP3D. It is based on defining a smooth (once continuously differentiable) trivariate function, $\mathrm{I}(\mathrm{x}, \mathrm{y}, \mathrm{z})$, which interpolates data values $\mathrm{F}$ scattered at scattered nodes. The interpolation scheme is a Modified Quadratic Shepard Method. The interpolant, I, is defined as

$$
I=\frac{\sum_{m=1}^{N N} W(m)^{*} I(m)}{\sum_{m=1}^{N N} W(m)}
$$

where $\mathrm{m}$ is the node index and $\mathrm{NN}$ is the number of nodes and their associated data values, a minimum of 10 such nodes should be used. The nodal function, $\mathrm{I}(\mathrm{m})$, at node $\mathrm{m}$ is given by 


$$
\begin{aligned}
& I(m)(x, y, z)= \\
& \quad A(1, m) * D X^{2}+A(2, m) * D X * D Y \\
& +A(3, m) * D Y^{2}+A(4, m) * D X * D Z \\
& +A(5, m) * D Y * D Z+A(6, m) * D Z^{2} \\
& +A(7, m) * D X+A(8, m) * D Y \\
& +A(9, m) * D Z \\
& +C(m)
\end{aligned}
$$

where

$$
\begin{aligned}
& D X=x-x(m) \\
& D Y=y-y(m) \\
& D Z=z-z(m)
\end{aligned}
$$

Thus $\mathrm{I}(\mathrm{m})$ is a quadratic function which interpolates the data values at node $\mathrm{m}$. The coefficients $(\mathrm{A}(1, \mathrm{~m}), \mathrm{A}(2, \mathrm{~m}) \ldots, \mathrm{A}(9, \mathrm{~m}))$ are obtained by a weighted least squares fit to the closest NI data points with weights similar to $\mathrm{W}(\mathrm{m})$, and using Gaussian Elimination to solve the resulting system of simultaneous equations. The recommended range of $\mathrm{NI}$ is between 16 and $\operatorname{Min}(40, \mathrm{NN}-1)$. The radius of influence, $R(m)$, for the least squares fit is fixed for each node $m$ but varies with $m$, and is chosen such that NW node are within the radius. The recommended value of NW is between 32 and $\operatorname{Min}(40, N N-1)$. The weights are taken to be

$W(m)(x, y, z)=\left[\frac{(R(m)-D(m))+}{R(m) * D(m)}\right]^{2}$

where

$(R(m)-D(m))+=0 \quad$ if $\quad R(m) \leq D(m)$

$\mathrm{D}(\mathrm{m})$ is the euclidean distance between node $(\mathrm{x}(\mathrm{m}), \mathrm{y}(\mathrm{m}), \mathrm{z}(\mathrm{m}))$ and cartesian location $(\mathrm{x}, \mathrm{y}, \mathrm{z})$. At a node $(x(m), y(m), z(m))$, weight $W(m)$ is not defined, however, $I(m)$ has limit $C(m)$. The use of such streamline to streamline mapping over streamline to gridblock mapping used before prevents unnecessary smoothing of saturation distribution and numerical dispersion.

\section{Application}

Synthetic Example 1. The TVD modification was validated using a synthetic example of a 2D, rectangular, homogeneous, quarter 5 spot pattern. A simulation grid of $21 \times 21 \times 1$ was used. The well configuration consisted of 1 injector and 1 producer. The simulation time is 500 days The producers are pressure constrained while the injector is rate constrained. The objective of presenting this example is to show the marked improvement in using TVD over other mathematical formulations like single point upstream weighting for the numerical solution along streamlines. To validate the TVD modification, the results of analytical solution and numerical 
simulation are also presented. Parameters used for comparison include oil rate versus time, water cut versus time, and saturation distribution at a reference time.

Synthetic Example 2. The modification for non-uniform initial conditions was validated using a synthetic example of pattern conversion from 5 spot to 9 spot. The synthetic example consisted of a $2 D$, rectangular, heterogeneous pattern with a simulation grid of $21 \times 21 \times 1$. The initial well configuration consists of 4 corner producers and 1 central injector. The simulation time is 1000 days while infill wells are introduced at 500 days. The infill wells are the 4 side producers. Fig.5 shows the well configuration for this example before and after infill. The producers are pressure constrained while the injector is rate constrained. Results of streamline simulation were compared with that of a commercial numerical simulator. The objective of presenting this example is to show the application of 3D interpolation and numerical solution along streamlines to model nonuniform initial conditions (infill drilling). Results of streamline simulation were compared with that of a commercial numerical simulator. Parameters used for comparison include oil rate versus time, and saturation distribution at a reference time (both at infill time and at the end of simulation)

Synthetic Example 3. This is a large scale, 2D, homogeneous, example with multiwell configuration. A simulation grid of $100 \times 50 \times 1$ was used to model multiple patterns containing 20 original producers, 15 injectors and 7 infill producers. This well configuration belongs to the North Robertson Field in West Texas. The producers are pressure constrained while the injectors are rate constrained. The reservoir was assumed closed at all the external boundaries. The total simulation time was 1000 days, with infill drilling at 700 days. The objective for presenting this case is to exhibit the response of streamline simulation to a multiwell configuration. Results of streamline simulation were compared with that of a commercial numerical simulator. Parameters used for comparison include oil rate versus time, water cut versus time, and saturation distribution at a reference time.

The approach is currently being applied to a large scale field example - the North Robertson Field in West Texas. A simulation grid of $100 \times 100 \times 12$ is being used to model multiple patterns totaling 20 original producers, 15 injectors and 7 infill producers. The producers are pressure constrained while the injectors are rate constrained. The reservoir is assumed closed at all the external boundaries. The total simulation time is 1000 days, with infill drilling at 700 days. The results of streamline simulation are being compared with available production history.

\section{Results and Discussion}

Synthetic Example 1. Fig.6 is a plot of oil rate vs. time for the streamline simulator and the numerical simulator. A very good match is seen between the streamline TVD solution, streamline analytical solution and the numerical simulation. The streamline 1 point upstream solution predicts a very early breakthrough in contrast. Fig. 7 is a plot of water cut vs. time for all 4 approaches. Fig.8 shows the water saturation maps from the streamline TVD solution along streamlines, streamline 1 point upstream solution streamlies, streamline analytical solution streamlines, and from numerical simulation. There is excellent agreement between the streamline simulator and the numerical simulator, except for the 1 point upstream solution in streamline simulation. 
Synthetic Example 2. Fig.9 is a plot of oil rate vs time for the streamline simulator and the numerical simulator. The results of well 1 (original producer) and well 5 (infill producer) have been plotted. A very good match between both the simulators for both wells. The breakthrough time is about 200 days in both simulators for well 1. Fig.10 is a plot of total oil rate vs time for the streamline simulator and the numerical simulator. Total oil rate is the sum of all the 4 producers before infill and sum of all the 8 producers after infill. Fig.11 shows the permeability map, and water saturation maps at infill time (300 Days, 0.425 PV Injected) and at the end of simulation (500 Days, $0.708 \mathrm{PV}$ Injected) from both the streamline simulator and the numerical simulator. The streamline simulator water saturation profile honors the permeability distribution very well. In addition, there is agreement between the streamline simulator and the numerical simulator. However, the numerical simulator does show the effects of dispersion as evident from the smoothing of contours.

Synthetic Example 3. Fig.12 is a plot of oil rate vs time for the streamline simulator and the numerical simulator. The results of well 1 (original producer) and well 21 (infill producer) have been plotted. A very good match between both the simulators for both wells. The breakthrough time is about 500 days in both simulators for well 1. Fig.13 is a plot of total oil rate vs time for the streamline simulator and the numerical simulator. Total oil rate is the sum of all the 20 producers before infill and sum of all the 27 producers after infill. Fig.14 shows water saturation maps at infill time (700 days, 0.157 PV Injected) and at the end of simulation (1000 Days, 0.224 PV Injected) from both the streamline simulator and the numerical simulator. There is a good agreement between the streamline simulator and the numerical simulator.

\section{Summary and Conclusions}

1. A fully three-dimensional streamline simulator has been developed for modeling multiphase flow in heterogeneous permeable media. The model is very general and orders of magnitude faster compared to traditional numerical simulators without any loss in accuracy.

2. The Total Variation Diminishing technique for the water saturation solution along streamlines is very effective in minimizing numerical dispersion, as evident from the results of synthetic example 1. It agrees very well with the analytical solution. In comparison, the solution from 1 point upstream solution along streamlines predicts early breakthrough due to numerical dispersion.

3. The modification for non-uniform initial conditions has been validated against numerical solution as shown in the results from synthetic example 2. The streamline simulator produces similar results to the numerical simulation for the infill wells using a fraction of the computation time.

4. The streamline approach is applicable to multiwell configuration, as evident from the results of synthetic example 3. The streamline simulation results agree with the numerical simulation for both individual wells and total reservoir rate. 


\section{Nomenclature}

a = slope of velocity components, $T^{-1}, 1 / \mathrm{sec}$

$\mathrm{B}_{\mathrm{o}}=$ oil formation volume factor,dimensionless, $\mathrm{rcf} / \mathrm{scf}$

$\mathrm{B}_{\mathrm{w}}=$ water formation volume factor, dimensionless, $\mathrm{rcf} / \mathrm{scf}$

$\mathrm{C}(\mathrm{m})=$ limit on interpolating function, dimensionles

$\mathrm{D}(\mathrm{m})=$ Euclidiean distance, $\mathrm{L}, \mathrm{ft}$

$\mathrm{DX}=\mathrm{x}$-direction distance of arbitrary point from a node, $\mathrm{L}, \mathrm{ft}$

$\mathrm{DY}=\mathrm{y}$-direction distance of arbitrary point from a node, $\mathrm{L}, \mathrm{ft}$

$\mathrm{DZ}=\mathrm{z}$-direction distance of arbitrary point from a node, $\mathrm{L}, \mathrm{ft}$

$\mathrm{dx}=$ distance traveled in $\mathrm{x}$ direction, $\mathrm{L}, \mathrm{ft}$

$\mathrm{dy}=$ distance traveled in $\mathrm{y}$ direction, $\mathrm{L}, \mathrm{ft}$

$\mathrm{dz}=$ distance traveled in $\mathrm{z}$ direction, $\mathrm{L}, \mathrm{ft}$

$\Delta \mathrm{x} \quad=$ gridblock size in $\mathrm{x}$-direction, $\mathrm{L}, \mathrm{ft}$

$\Delta y \quad=$ gridblock size in $\mathrm{y}$-direction, $\mathrm{L}, \mathrm{ft}$

$\Delta \mathrm{z} \quad=$ gridblock size in $\mathrm{z}$-direction, $\mathrm{L}, \mathrm{ft}$

$\Delta \mathrm{t}=$ small timestep in numerical ID solution, $\mathrm{T}$, days

$\mathrm{F} \quad=$ third order term, dimensionless

$\mathrm{f}_{\mathrm{w}} \quad$ fractional flow of water, dimensionless, fraction

$\mathrm{i} \quad=$ gridblock index

$\mathrm{I}(\mathrm{m})=$ Interpolant, dimensionless

$\mathrm{k}_{\mathrm{ro}} \quad=$ oil relative permeablity, dimensionless, fraction

$\mathrm{k}_{\mathrm{rw}} \quad=$ water relative permeablity, dimensionless, fraction

$\mathrm{k}_{\mathrm{ron}}=$ endpt. oil relative permeablity, dimensionless, fraction

$\mathrm{k}_{\mathrm{rwn}}=$ =ndpt. water rel. permeablity, dimensionless, fraction

$\mathrm{kx}=$ absolute permeability in $\mathrm{x}$-direction, $\mathrm{L}^{2}, \mathrm{md}$

ky $\quad$ absolute permeability in $\mathrm{y}$-direction, $\mathrm{L}^{2}$, $\mathrm{md}$

$\mathrm{kz}=$ absolute permeability in $\mathrm{z}$-direction, $\mathrm{L}^{2}, \mathrm{md}$

$1=$ counter for streamlines

$\mathrm{m} \quad=$ node counter

$\mathrm{M}_{\text {end }}=$ End point mobility ratio, dimensionless

$\mathrm{n}=$ timestep counter

N1D = number of gridblocks overlaid on each streamline

ngr $=$ net-to-gross ratio, dimensionless, fraction

NI = number of nodes

$\mathrm{NI}=$ Number of nodes used for determining coefficients

$\mathrm{NW}=$ Number of nodes within radius of influence

$\mathrm{n}_{\mathrm{o}} \quad=$ oil relative permeability exponent, dimensionless

$\mathrm{n}_{\mathrm{w}}=$ water relative permeability exponent,dimensionless

$\mathrm{Np}=$ cumulative oil production, $\mathrm{L}^{3}, \mathrm{stb}$

$\mathrm{N}_{\mathrm{sl}}$ = number of streamlines to a producer, dimensionless

$\mathrm{p} \quad=$ pressure, $\mathrm{M} / \mathrm{L}^{2}, \mathrm{psi}$

$\mathrm{PV}=$ pore volume, $\mathrm{L}^{3}, \mathrm{rcf}$

$\mathrm{q}=$ specific flow rate, $\mathrm{T}^{-1}, 1 / \mathrm{d}$

$\mathrm{Q}=$ flow rate, $\mathrm{L}^{3}, \mathrm{stb} / \mathrm{d}$

$\mathrm{Q}_{\mathrm{t}}=$ total flow rate, $\mathrm{L}^{3}, \mathrm{stb} / \mathrm{d}$ 


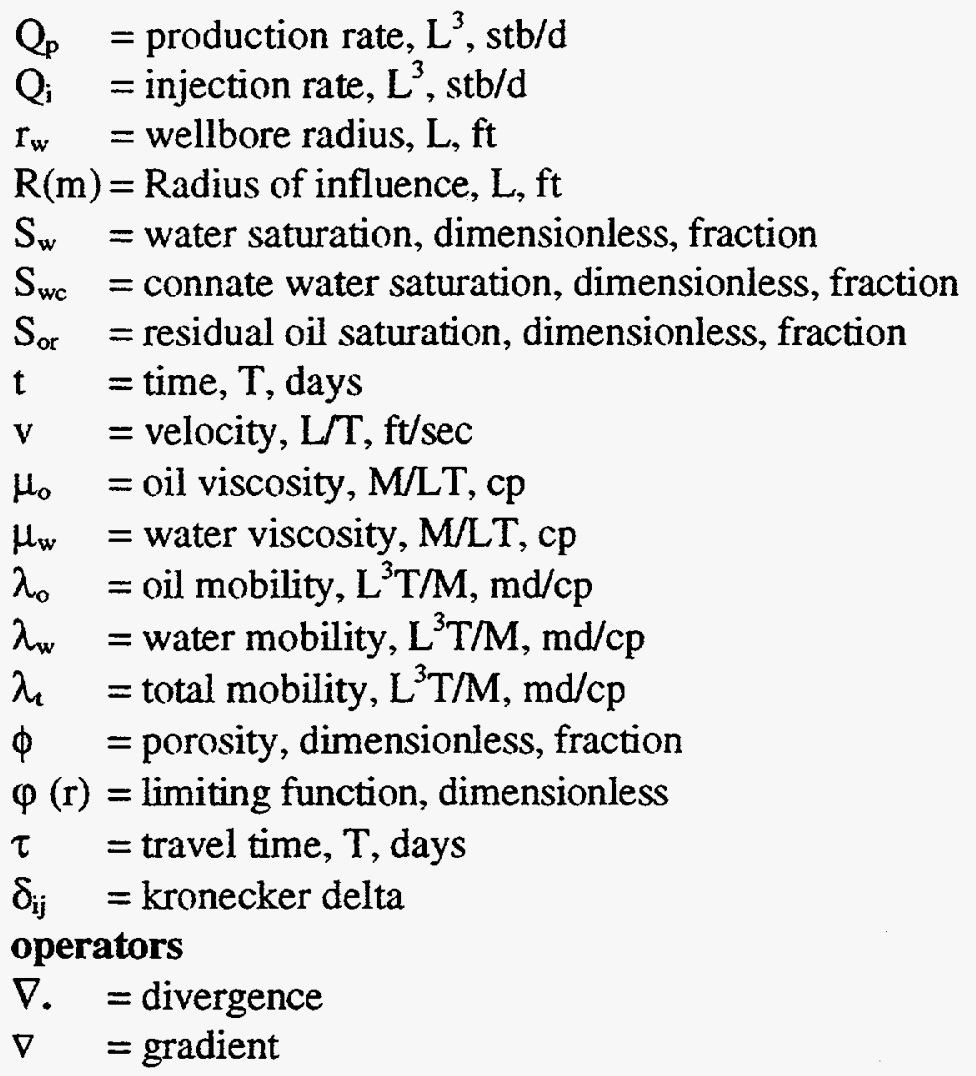

\section{References}

1. Datta-Gupta A. and King M.J.: "A Semianalytic Approach to tracer Flow Modeling in Heterogeneous Permeable Media," Advances in Water Resources 18 (1995) 9-24.

2. Peddibhotla S., Cubillos H.G., Datta-Gupta A., and Wu C.H.: "Rapid Simulation of Multiphase Flow Through Fine-Scale Geostatistical Realizations Using a New, 3D, Streamline Model: A Field Example", SPE 36008, Proceedings of the $11^{\text {th }}$ SPE Petroleum Computer Conference, Dallas (June 1996).

3. Peddibhotla S., Datta-Gupta A., and Xue G.: "Multiphase Streamline Modeling in Three Dimensions: Further Generalizations and a Large Scale Field Application", SPE 38003, Proceedings of the $14^{\text {th }}$ SPE Reservoir Simulation Symposium, Dallas (June 1997).

4. Peddibhotla S., Spath J.B., and Batycky R.P.: "An Efficient PC Based Streamline Simulator for Immiscible and Miscible Displacements", SPE 38129, Proceedings of the $12^{\text {th }}$ SPE Petroleum Computer Conference, Dallas (June 1997).

5. Vasco D.W., Datta-Gupta A. and Long J.C.S. "Integrating Field Production History in Stochastic Reservoir Characterization", SPE 36567, Proceedings of the $70^{\text {th }}$ SPE Annual Technical Conference \& Exhibition, Denver (October 1996).

6. Buckley S.E. and Leverett M.C.: "Mechanism of Fluid Displacement in Sands", Trans. AIME (1941), 249, Pg. 107.

7. Wang B., Lake L.W. and Pope G.A.: "Development and Application of a Streamline Miscellar Polymer Flooding", SPE 10290, Proceedings of $56^{\text {th }}$ Annual Technical Conference \& Exhibition, SanAntonio (October 1981).

8. Harten A.: "High Resolution Schemes for Hyperbolic Conservation Laws", Journal of Computational Physics, Vol.49, Pg.357 (1983).

9. Datta-Gupta A., Lake L.W., Pope G.A., Sepehrnoori K., and King M.J.: "High Resolution Monotonic 
Schemes for Reservoir Fluid Flow Simulation", IN SITU, Vol.15, No.3, Pg.289 (1991).

10. Leonard B.P.: "Universal Limiter for Transient Interpolation Modeling of the Advective Transport Equations: The ULTIMATE Conservative Difference Scheme", NASA TM 100916, ICOMP-88-11 (1988).

11. Sweby P.K.: "High Resolution Schemes using Flux Limiters for Hyperbolic Conservation Laws", SIAM Journal of Numerical Analysis, Vol.21, Pg.995 (1990).

12. Craig F. F. Jr.: "The Reservoir Engineering Aspects of Waterflooding", Monograph Series, SPE, Richardson, TX (1973).

13. Batycky R.P., Blunt M.J., and Thiele M.R.: “A 3D Field Scale Streamline Simulator With Gravity and Changing Well Conditions", SPE 36726, Proceedings of the SPE ATC\&E, Denver (October 1996).

14. Renka R.J.: "QSHEP3D: Quadratic Shepard Method for Traivariate Interpolation of Scattered Data", ACM Transactions on Mathematical Software, Vol.14, No.2, Pg.151 (June 1988).

15. Renka R.J.: "QSHEP2D: Quadratic Shepard Method for Traivariate Interpolation of Scattered Data", ACM Transactions on Mathematical Software, Vol.14, No.2, Pg.149 (June 1988).

16. Corey A.T.: "The Interrelation Between Gas and Oil Relative Permeabilities", Producers Monthly (1954) 19, No 1, Pg.38.

17. Martin J.C. and Wagner R.E.: "Numerical Solution of Multiphase Flow Using Stream-Tube Relationships", SPE 7140, Proceedings of the $49^{\text {th }}$ California Regional Meeting, San Fransisco (April 1978).

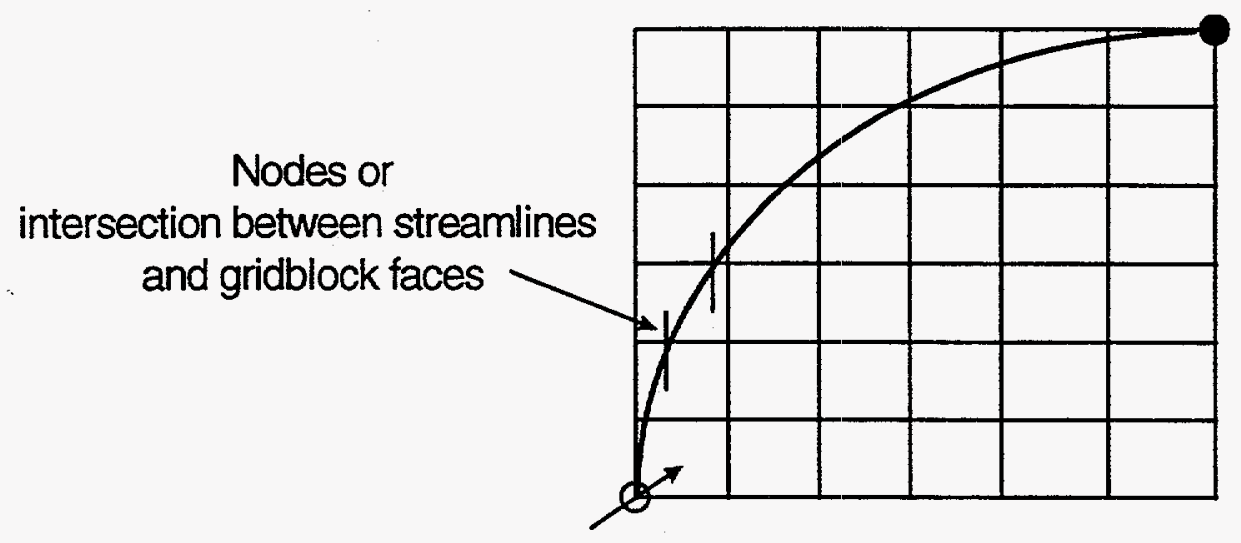

Fig.1 - Streamlines between injectors and producers. 


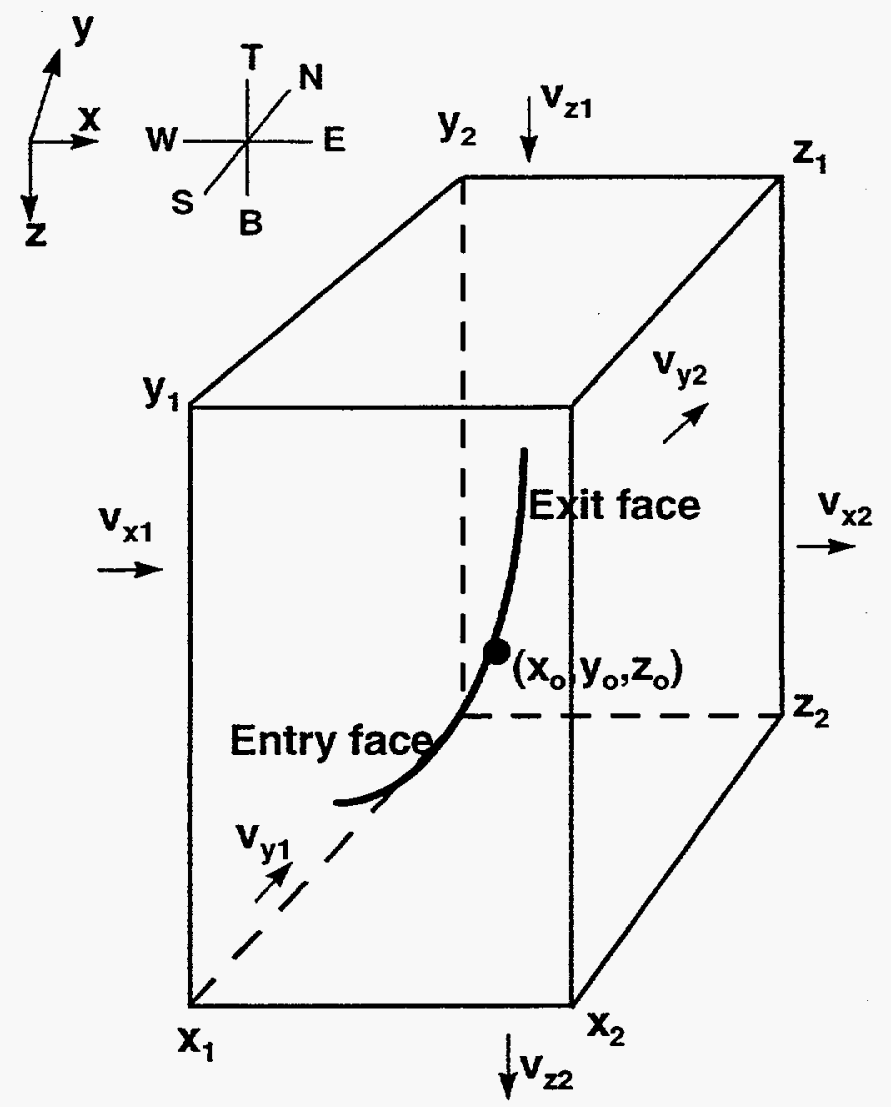

Fig.2 - Streamline passing through a gridblock.

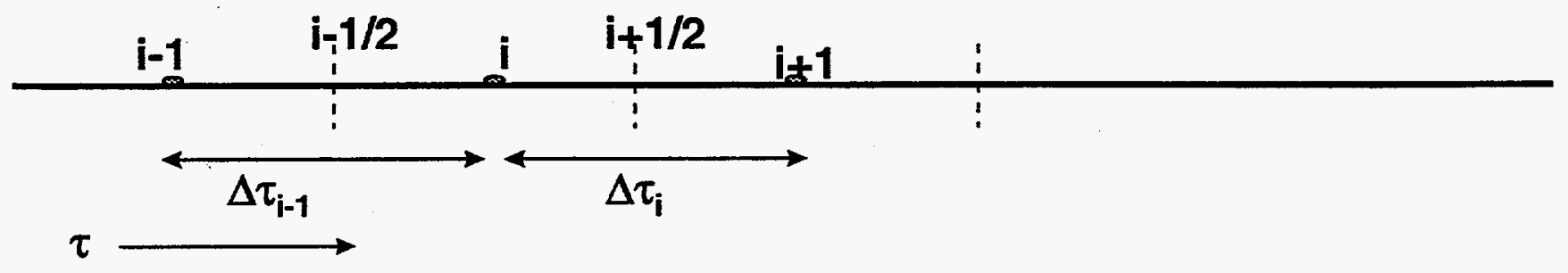

Fig.3 - 1-D solution along streamline. 


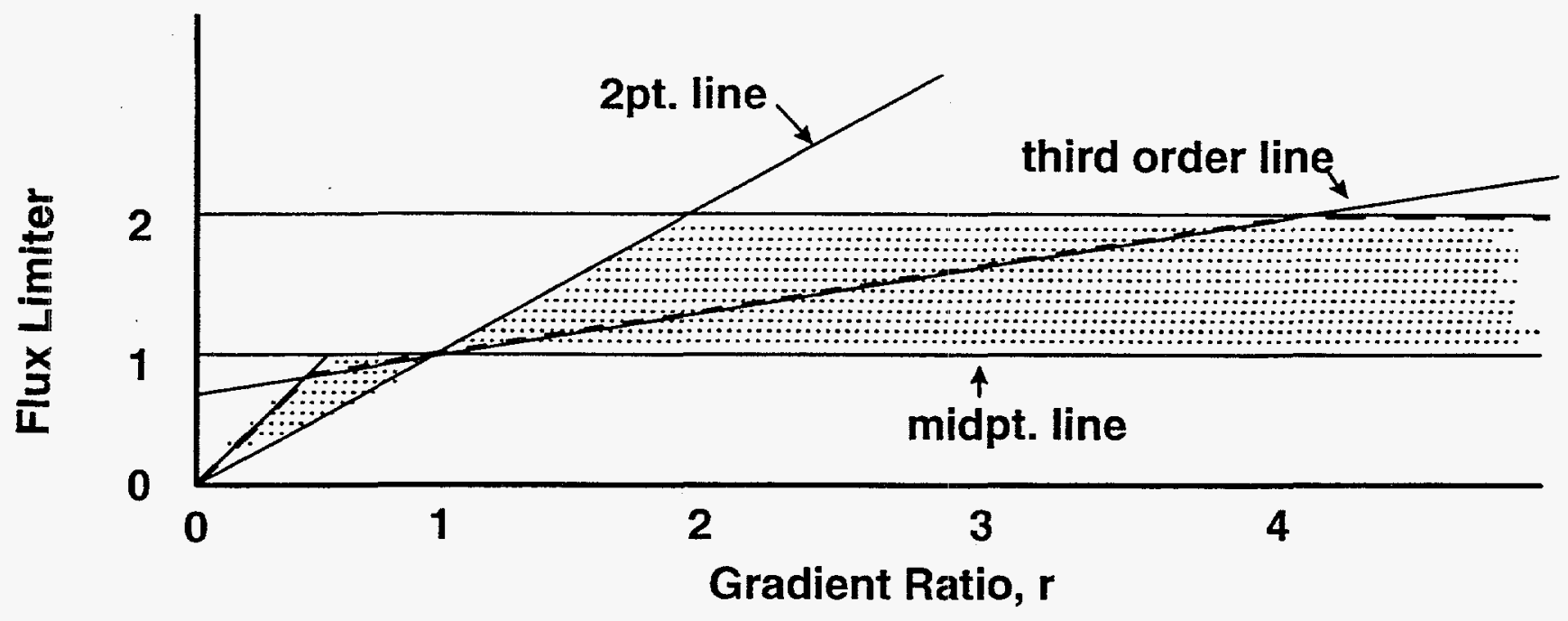

Fig.4 - Second order TVD region (shaded area) showing the third order lines for Leonard (solid line) and TVD Leonard (dashed bold line).

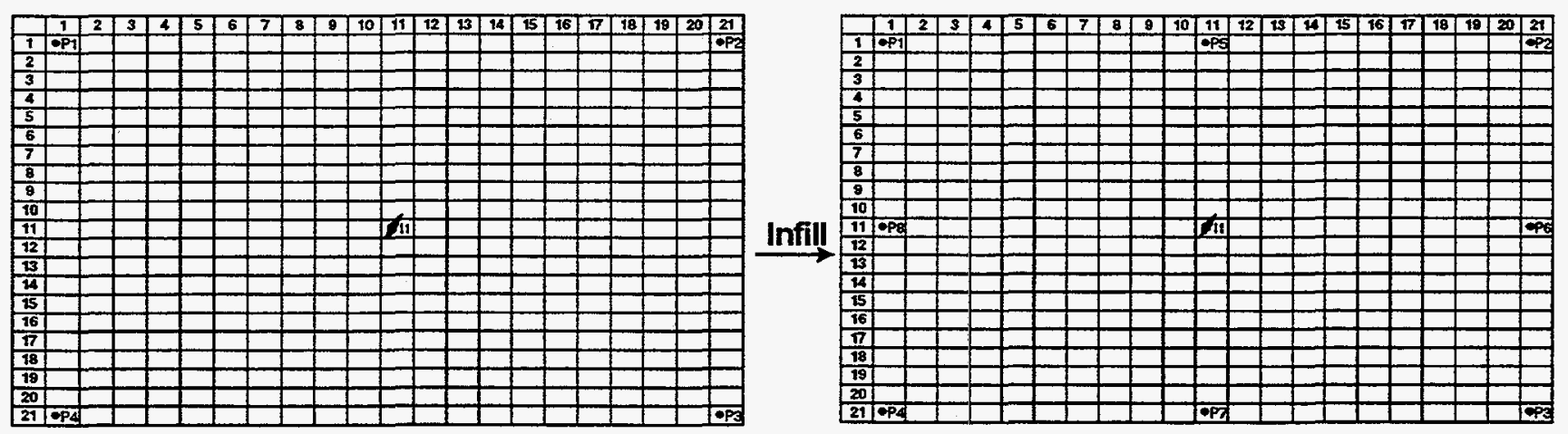

Fig.5 - Well Configuration for synthetic example 2.

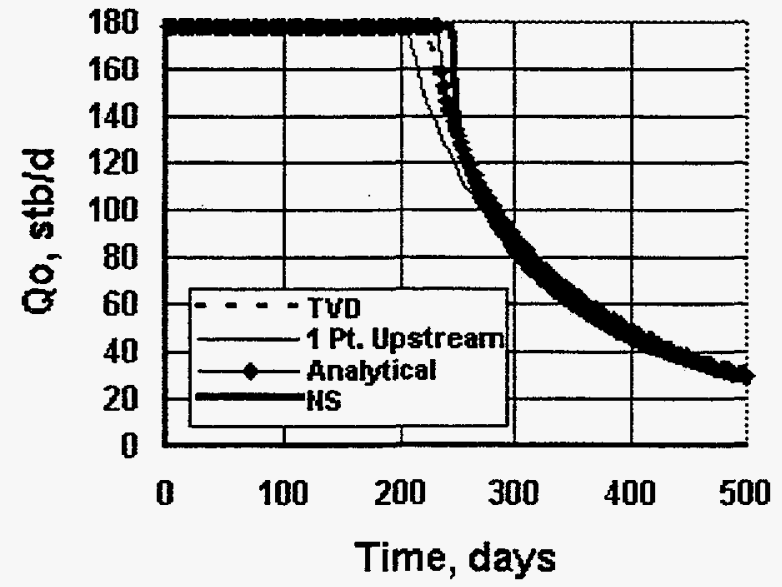

Fig.6-Oil rate vs. time for synthetic example 1.

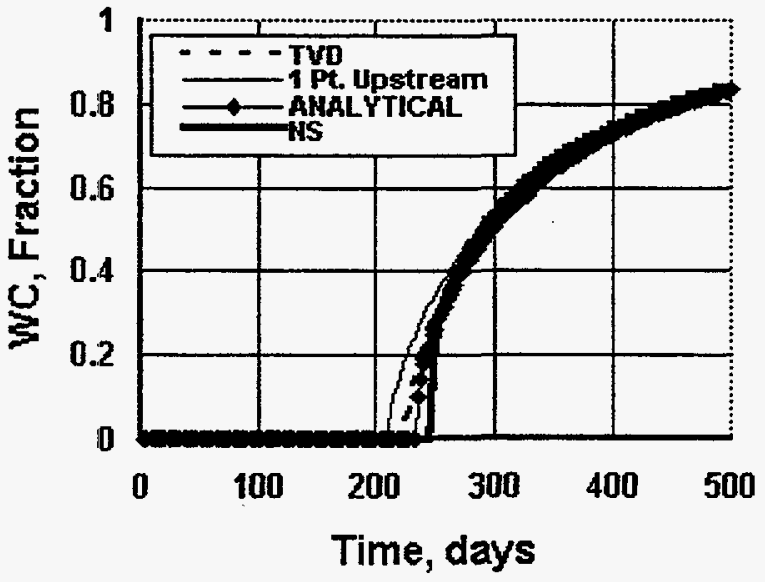

Fig.7-Water cut vs. time for synthetic example 1. 

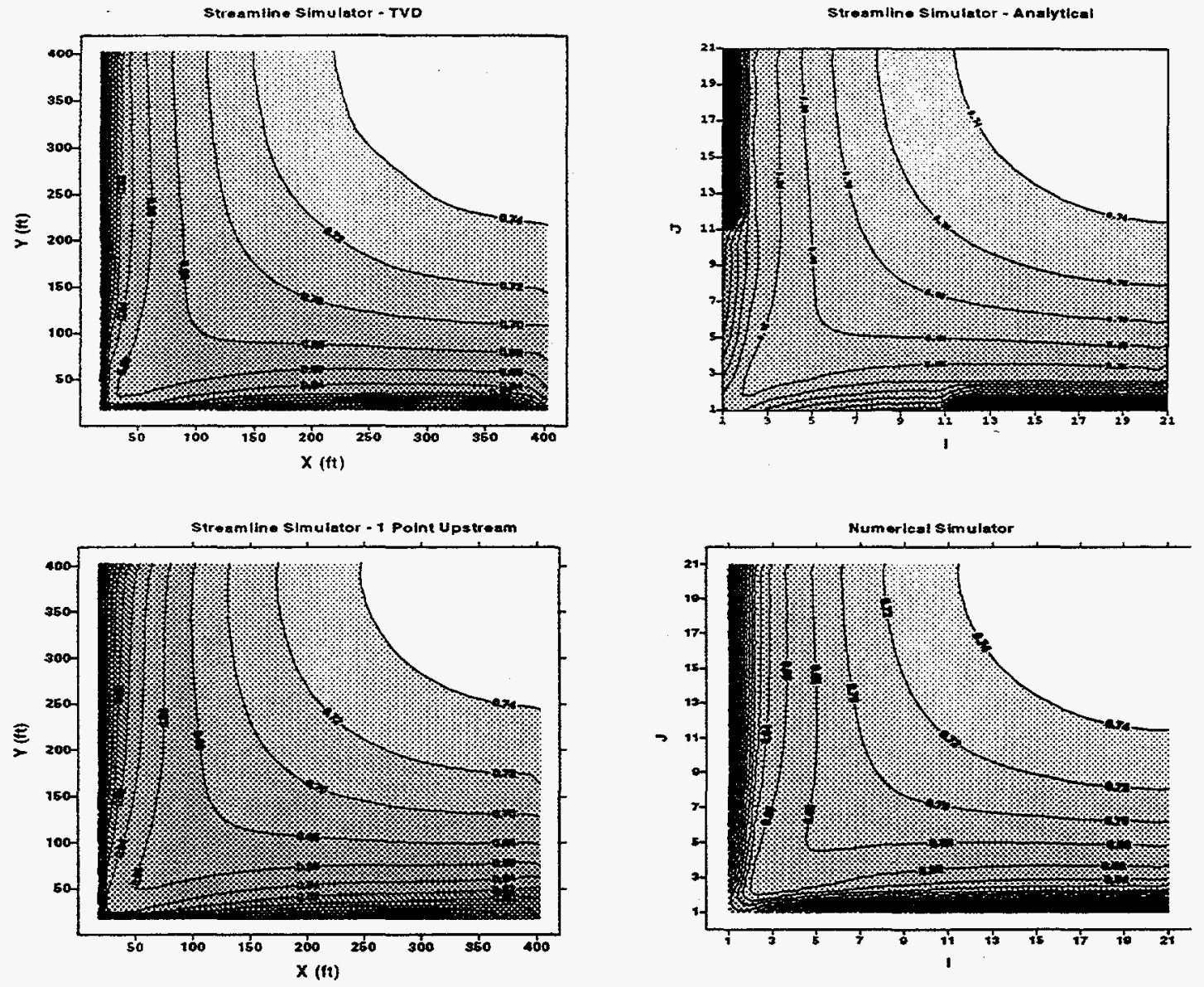

Fig.8 - Water saturation contour maps at $\mathbf{3 0 0}$ days for synthetic example 1.

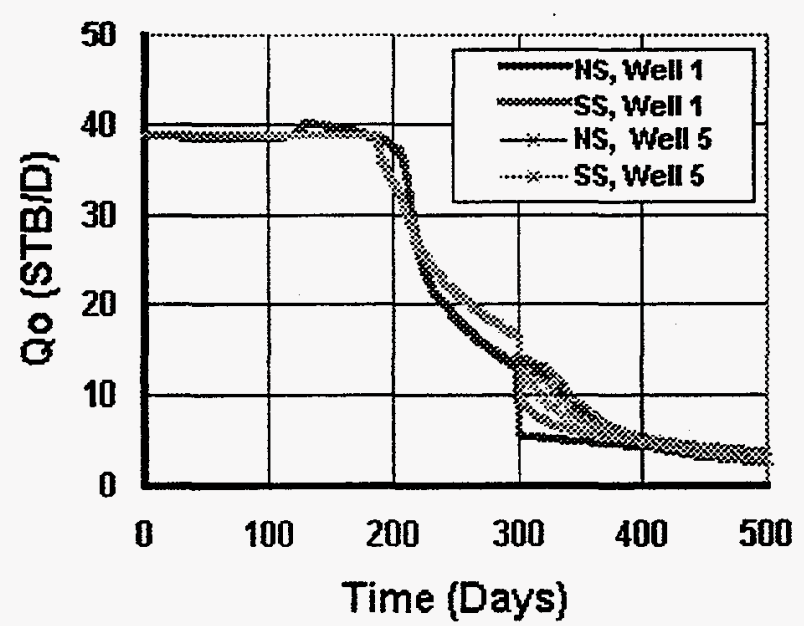

Fig.9-Oil rate vs. time for synthetic example 2.

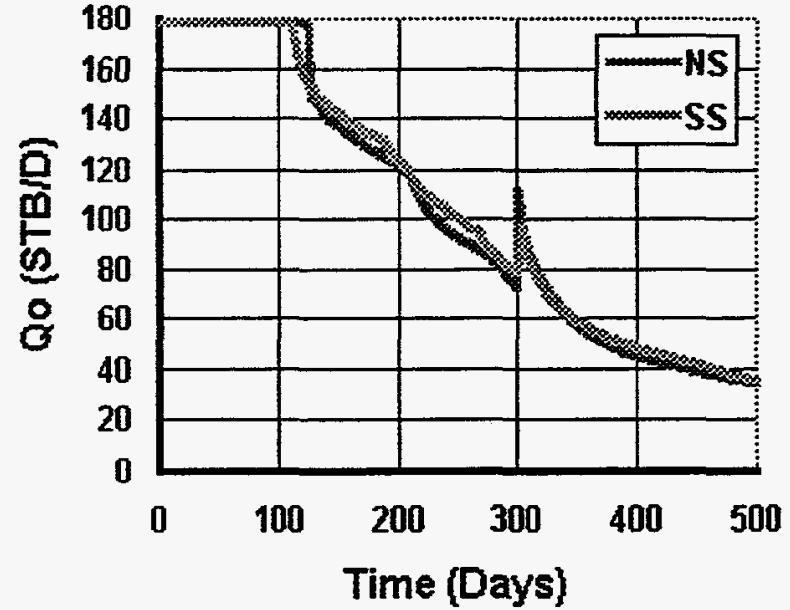

Fig.10-Total rate vs. time for synthetic example 2. 


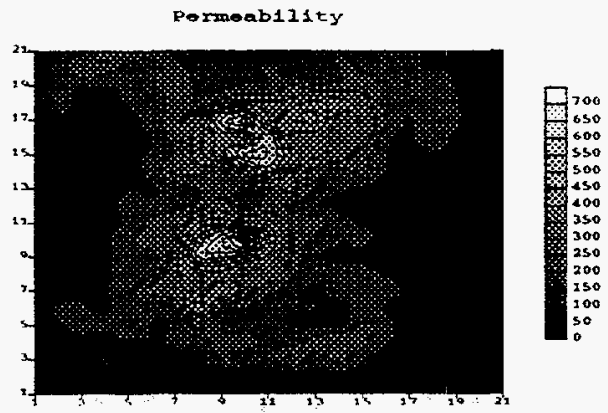

At Infill Time (300 Daye)

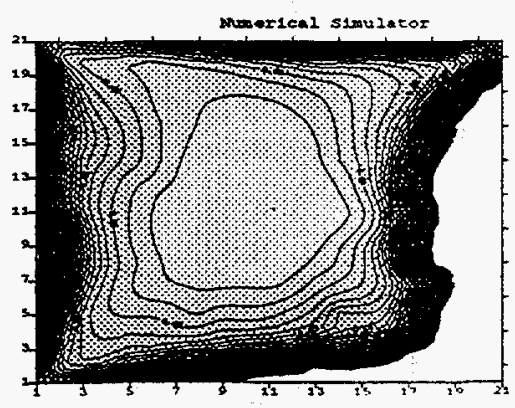

stremel10 stmulator

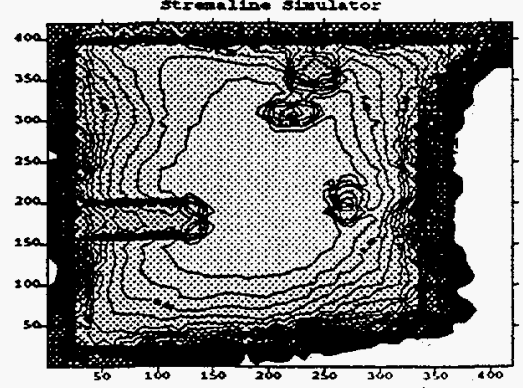

At End of stmulation (500 Deys)

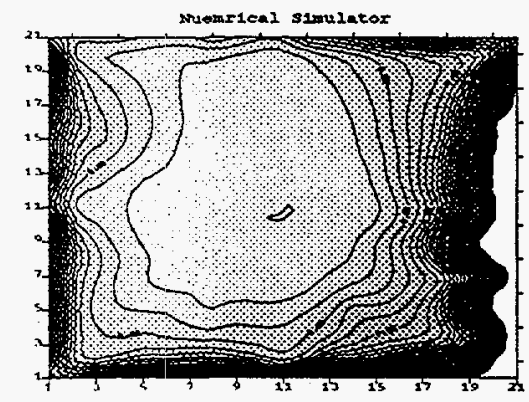

Strandine ssmulator

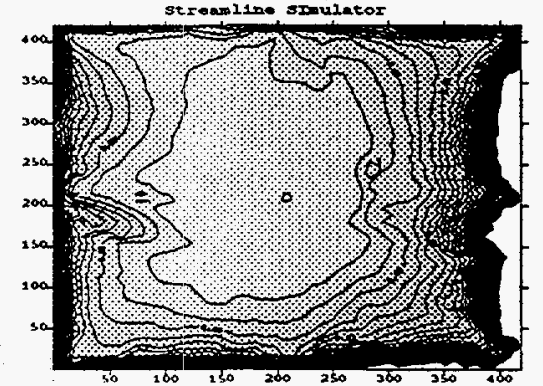

Fig.11 - Water saturation contour maps for synthetic example 2. 


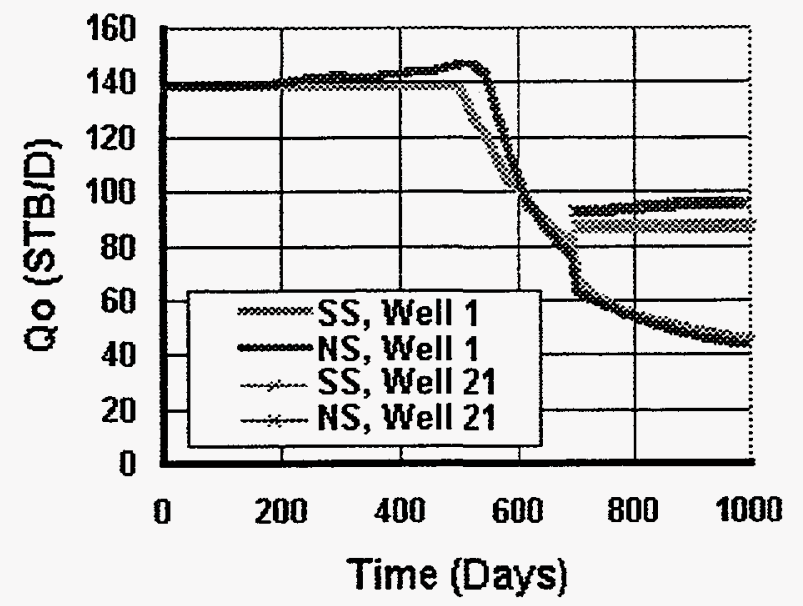

Fig.12-Oil rate vs. time for synthetic example 3.

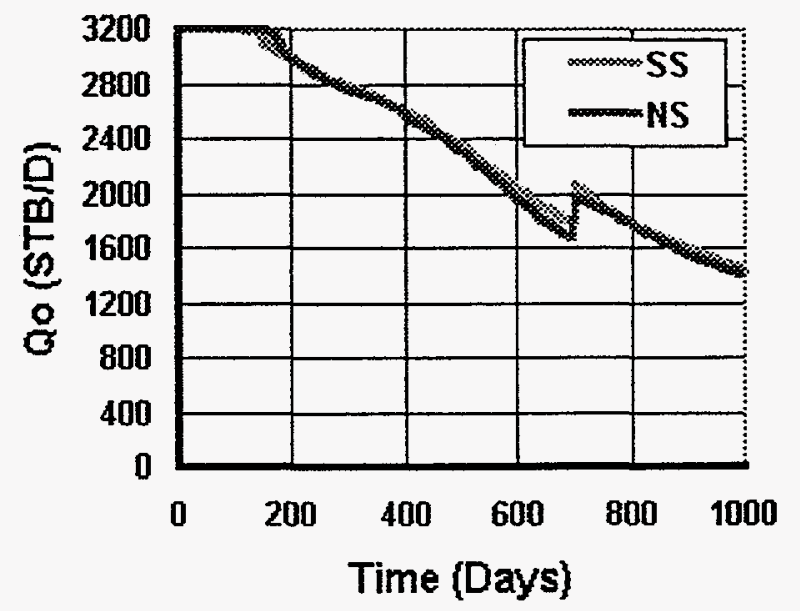

Fig.13-Total rate vs. time for synthetic example 3.

\section{At Infill Time (700 Days)}
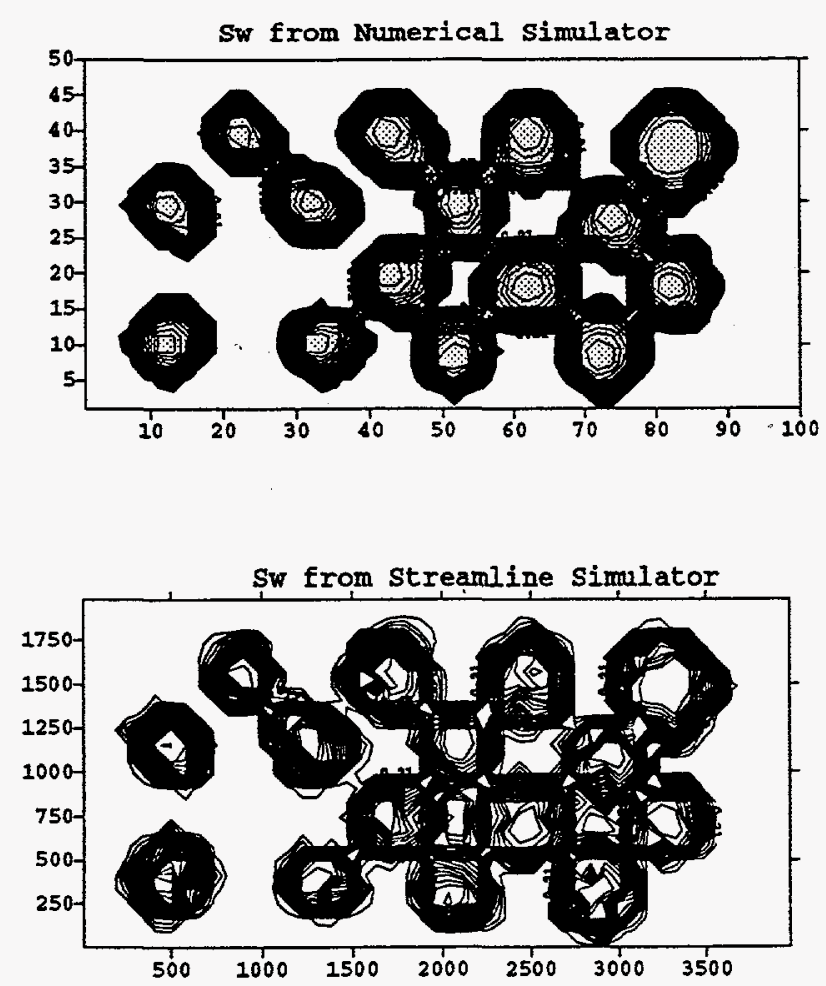

At End of Simulation (1000 Days)
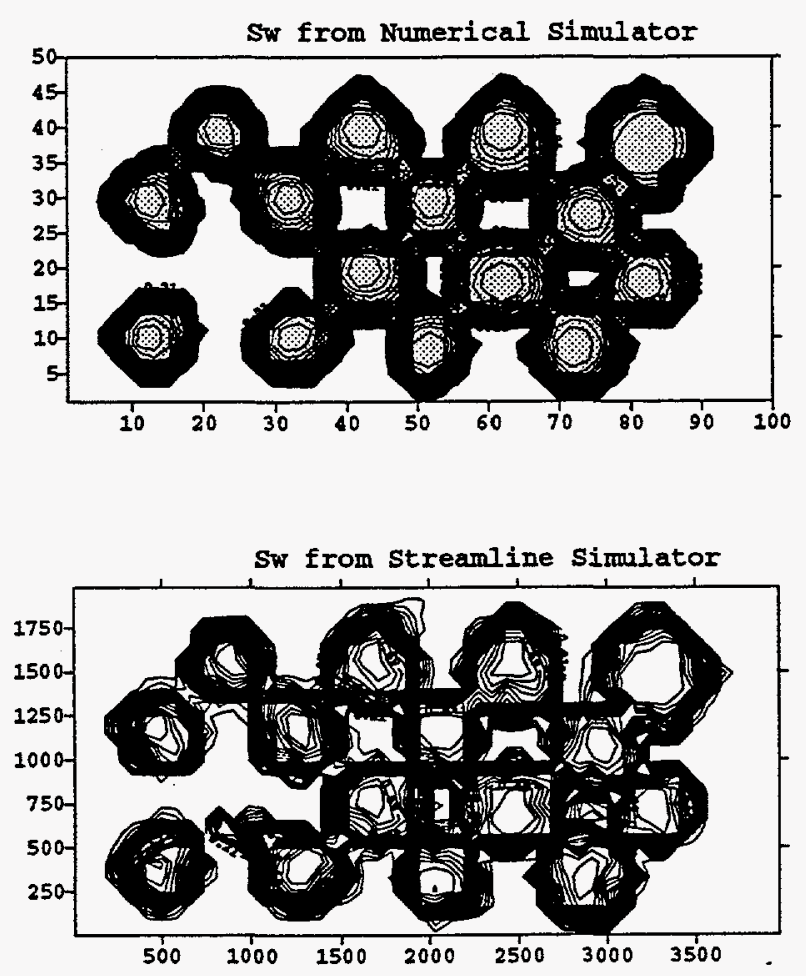

Fig.14 - Water saturation contour maps for synthetic example 3. 


\section{B. Application of the Theory to La Cira Field in Colombia}

A geostatistical approach is commonly used to reproduce reservoir heterogeneities. The objective is to generate a few "typical" descriptions incorporating heterogeneity elements that are difficult to include by conventional methods. Conditional simulation is used for creating property (permeability, porosity, etc.) distribution with a prescribed spatial correlation structure that honors measured data at well locations. Stochastic reservoir modeling provides multiple equiprobable, reservoir models, all data intensive, rather than a single, smooth usually data poor deterministic model. Experience has shown that these data intensive, stochastic reservoir models yield a better history match of production data, yet provide a measure of uncertainty in prediction of future performance.

Fine-scale realizations are the most detailed representation of the heterogeneities that exist in the petroleum reservoir. The ideal flow simulation process would be to input this fine-scale data in its entirety. However conventional numerical simulators do not allow this readily. Reservoir models built for conventional simulators using the fine-scale data are huge and unmanageable. The flow simulation process thus becomes very tedious, slow and expensive. This is in addition to any hardware limitations that may exist. Typically an upscaling algorithm is applied to obtain a coarsescale heterogeneity model. This coarse-scale model is then input into the conventional simulators. However, most of the upscaling algorithms are based on single phase pressure solution and are thus questionable at best for multiphase flow applications. Pseudo-relative permeabilities have often been used as a tool for multiphase flow upscaling. But such approaches are highly process dependent and have limited applicability. There is a definite need for a fast and powerful simulator that allows the easy use of fine-scale realizations as such without the need for any upscaling.

In this work, we describe application of the new, fully three-dimensional, multiphase, streamline simulator for modeling waterflood performance. The streamline simulator is orders of magnitude faster than traditional numerical simulators, and its performance is not affected by problems of numerical dispersion or instability. Unlike streamtube models, the proposed approach relies on the observation that in a velocity field derived by finite difference, streamlines can be approximated as piecewise hyperbolas within gridblocks. Thus the method can be easily applied in three-dimensions, and incorporated into conventional finite difference simulators. Once streamlines are generated in three dimensions, a variety of one dimensional problems can be solved analytically along the streamlines.

This is the first truly three dimensional streamline simulator applied to field level simulation. Till now, streamline simulation has been applied to three-dimensions by modeling the reservoir as a composite of two-dimensional layers or through hybrid approaches. The speed of the streamline simulator allows the dynamic flow simulation of multiple fine-scale realizations of reservoir properties and virtually eliminates the need for upscaling. The simulator can be used to assess the uncertainty associated with stochastic reservoir descriptions. The approach provides a lot of scope for future works, like combining dynamic field history data with static data for better reservoir description. 
The power and utility of the streamline simulator is demonstrated through application to a field example. The field example consists of a detailed characterization and waterflood performance prediction for the La Cira field, Colombia. Using core and log data, a fine-scale geostatistical description consisting of 100 layers was generated for a typical waterflood pattern. Waterflood performance prediction was carried out for 23 years of secondary production. The parameters used for comparison between the streamline simulator and commercial simulator are water cut vs. time, oil rate vs. time, and water saturation distribution. The streamline simulator is found to be orders of magnitude faster than the commercial simulator without any significant loss in accuracy.

The La Cira field is located in the Magdalena middle basin, Colombia, South America as illustrated in Fig.1. The field was discovered in 1926, and produced under the solution-gas drive mechanism until 1956 when a waterflooding project was started. The geological model was already available which describes the morphology of the sand bodies. The reservoir rock type is fluvial sandstone of the Eocene-Oligocene age. The sand bodies can be characterized as channel deposits of the jigsaw-puzzle reservoir type. The field structure is an anticline, nine kilometers by six kilometers, with the major axis N-S trending. The reservoir is classified as a low to medium permeability reservoir. The La Cira field produces from three zones - A, B and C. The C zone is the main producing zone yielding about $80 \%$ of the cumulative oil production. The field has been divided into eight areas for operations management. The OOIP was $1500 \mathrm{MMSTB}$, the primary recovery was about $11 \%$ and the current average recovery about $16 \%$. This analysis here will focus on a typical pattern (LC - 1210) in producing area 07.

In this section, we describe the distribution of permeability within the sand bodies. A conditional, geostatistical technique called Sequential Gaussian Simulation (SGS) was used to generate fine-scales realizations of permeability. The study was performed on a typical waterflood pattern (LC 1210) of production area 07, with an area of 43 acres, with 5 injectors and 1 central producer as illustrated in Fig.2. Conditional 3D permeability descriptions were generated for the same volume as in the dynamic simulation model. The origin, orientation, and external dimensions of the grid were tailored to the future fluid flow model grid constraints. In the study unit, the reservoir is composed of 6 layers, hydraulically separated but producing commingled; consequently all six layers have to be included in the fluid flow model. However, from the geostatistical point of view, the 6 pools (divided by impermeable shale) were simulated as 100 layers using a foot-by-foot description available from well logs and limited core data. The stochastic simulations were carried out for 100 layers, in a very detailed, orthogonal grid to obtain a fine-scale heterogeneity description. The grid dimensions for all layers in the fine scale simulation are $89 \mathrm{ft} . \mathrm{x} 94 \mathrm{ft}$. $\mathrm{x} 1 \mathrm{ft}$. Conditional simulation of permeability was carried out using an anisotropic semi-variogram model. Two nested structures and an isotropic nugget effect of 0.06 characterize the variogram model. The first structure is an exponential model, with a range in the direction of principal continuity (horizontal) of $6000 \mathrm{ft}$., with a contribution of 0.62 and an anisotropy factor of 0.0026 . The second structure is a Gaussian model, with a range in the direction of principal continuity (horizontal) of $4700 \mathrm{ft}$, with a contribution of 0.32 and an anisotropy factor of 0.0023 . The logarithm of permeability (conditioning data) is input foot-by-foot in all 6 well locations.

This fine-scale description can be used directly in the streamline simulator. Unfortunately, use of this reservoir model of 50400 gridblocks $(21 \times 24 \times 100)$ in a commercial 
numerical simulator turned out to be quite cumbersome because of the large storage and CPU time required. An upscaling procedure was therefore necessary to capture the effect of heterogeneities and to transfer it into a coarse grid for the commercial numerical simulation model. The upscaled permeability field is shown in Fig.3. The upscaling was necessary to be able to compare the streamline simulator with the numerical simulator. For permeability upscaling, the power averaging technique was used. This approach models the non-linear averaging of absolute permeabilities. The assumption is that the elementary permeability values average linearly after a non-linear power transformation. Multiple fine-scale stochastic realizations were generated by changing only the random seed number in the SGS.

La Cira field coarse-scale model. Further comparison between the streamline and commercial simulators was performed for a field case, the La Cira field in Colombia, South America. The course-scale permeability model obtained from upscaling was used here. The case consisted of five injectors and one central producer in a 3-D, rectangular, heterogeneous model $(21 \times 24 \times 6)$ of a typical waterflood pattern LC 1210, Area 07, La Cira field, Colombia. The upscaled permeability field consisting of 6 layers shown in Fig.4, was used with $\mathrm{kx}=\mathrm{ky}$ in each gridblock. Performance predictions for 23 years of secondary production was carried out using both the streamline simulator and the commercial simulator. The parameters used for comparing the streamline simulator with the commercial simulator are oil rate vs. time and spatial water saturation distribution at a particular time of reference.

La Cira field coarse-scale model. Fig.4 is a plot of oil rate vs. time for the streamline simulator, commercial simulator and actual production history. The match between the streamline simulator and the actual production history using the upscaled permeability description is not very satisfactory. The match between the commercial simulator and the actual production history is not good either. Both the simulators tend to under predict the oil rate at all times. There is an initial hump in oil rate predicted by the commercial simulator. This can be attributed to the fill-up volume associated with the free gas saturation in the reservoir. The streamline model is not able to model such fill-up period due to the incompressibility assumptions. After 8000 days of water injection $(0.59$ PV injected); both simulators gave a water cut of 0.98 . A comparison of CPU times between the streamline simulator and the commercial simulator shows that the streamline simulator is about 200 times faster than the commercial simulator.

1. We have presented a fully three dimensional streamline simulator for predicting field-scale waterflood performance.

2. The streamline simulator has been shown to be orders of magnitude faster compared to the commercial simulator. This allows for use of fine-scale description, virtually eliminating the need for any upscaling.

3. Comparisons with a commercial simulator have shown that the streamline simulator yields satisfactory results for typical waterflood conditions. Also, unlike the commercial simulator the streamline approach does not suffer from numerical dispersion or instabilities.

4. The power and utility of the streamline simulator has been demonstrated through application to waterflood performance for the La Cira field, Colombia. 


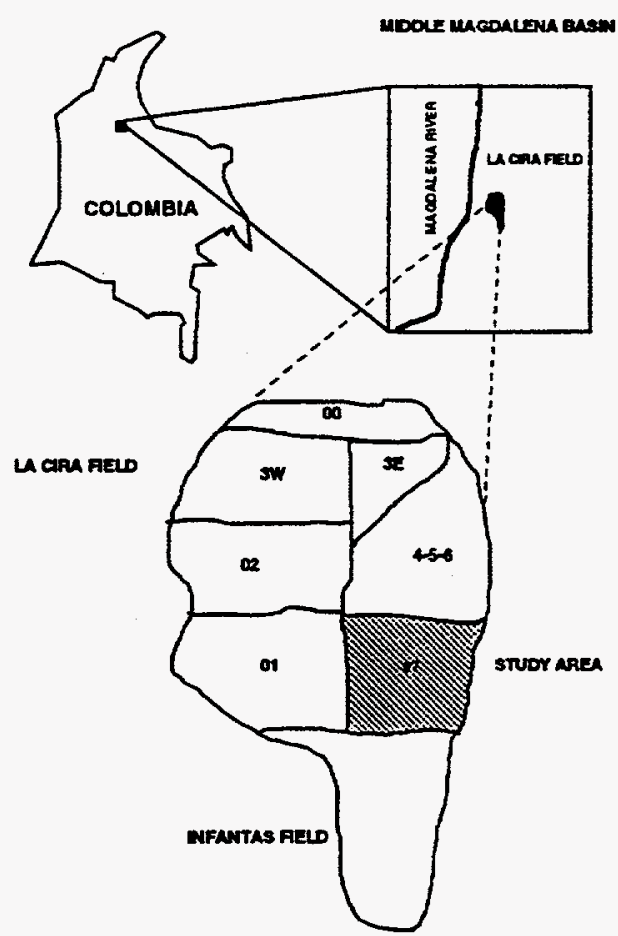

Fig. 1-Location of La Cira field.

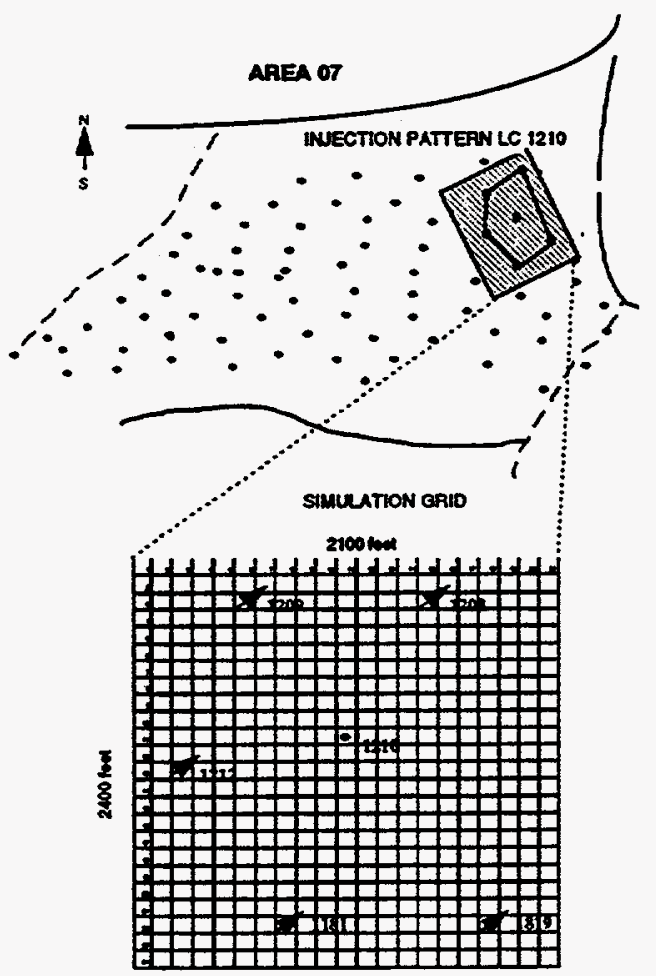

Fig. 2-Waterflood pattern. 


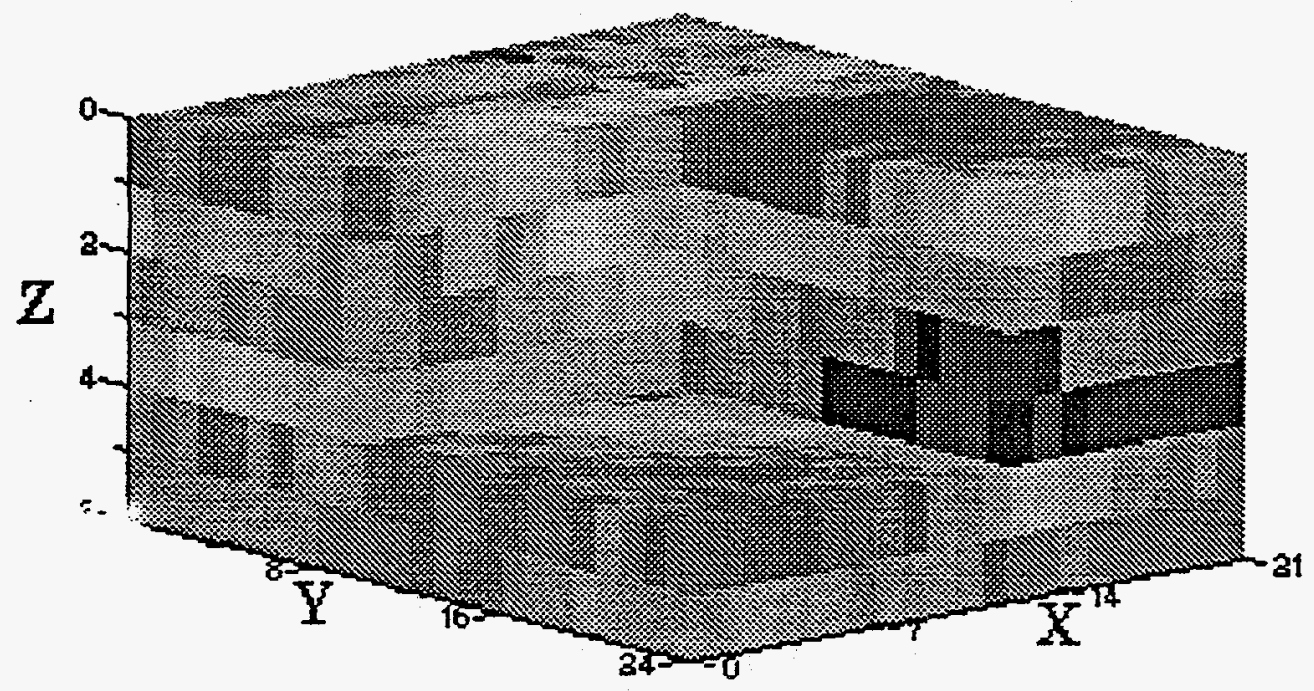

Color Mapping

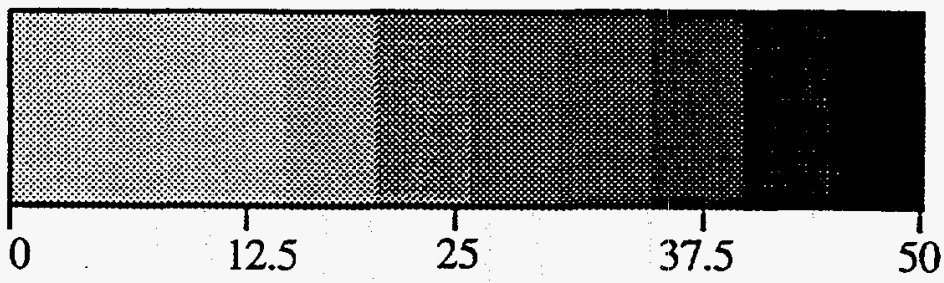

Fig. 3-Coarse-scale geostatistical permeability model.

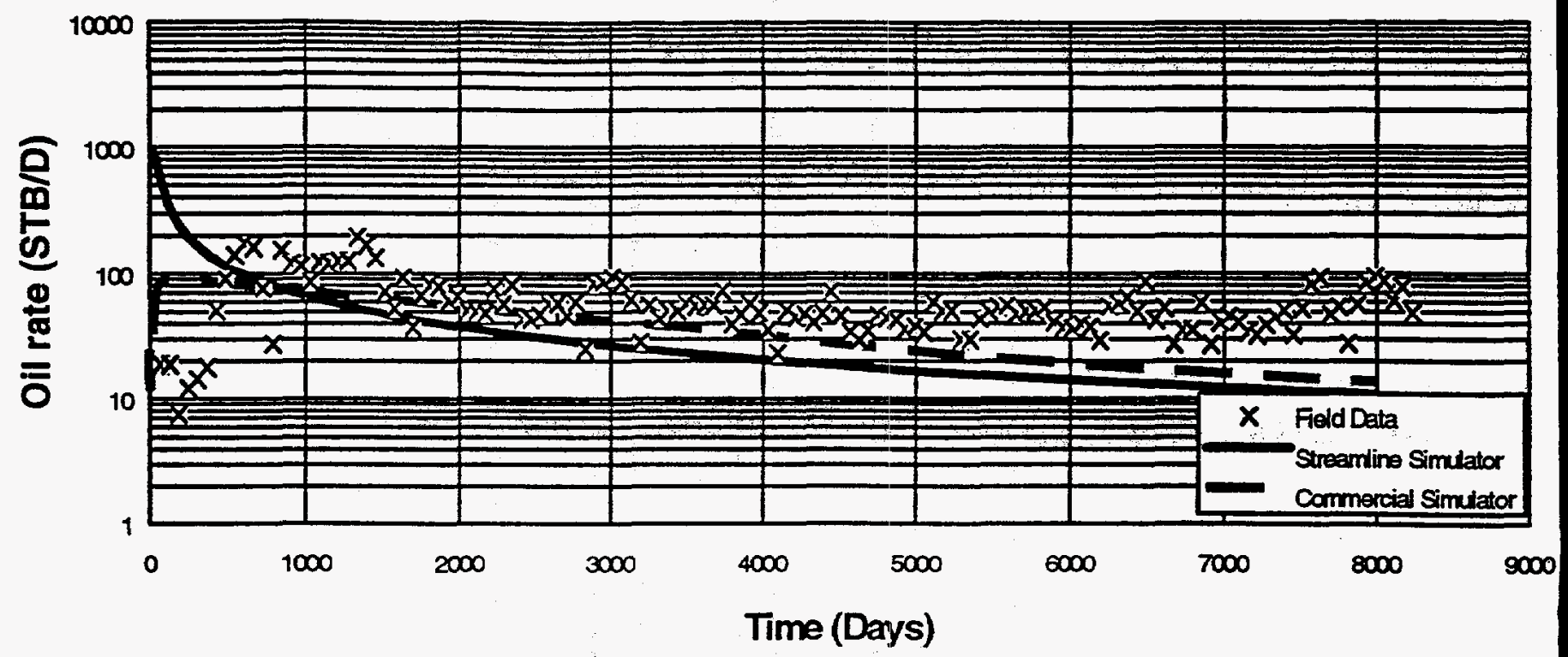

Fig. 4-Oil rate vs. time. 


\section{SEISMIC SIGNATURES OF THE FRACTURED TWIN CREEK RESERVOIR, LODGEPOLE FIELD, UTAH-WYOMING OVERTHRUST BELT}

\section{A. Introduction}

In low porosity, low permeability formations, natural fractures are the primary source of permeability, controlling both production and injection of fluids. The open fractures do not contribute significantly to total porosity, but they provide an increased drainage network for the matrix porosity. An important approach to characterizing the fracture orientation and fracture permeability of reservoirs is one based upon the effects of such conditions on the propagation of acoustic and seismic waves in the rock.

We present the feasibility of using seismic measurement techniques to map the fracture zones between wells spaced 2,400 feet apart at a depth of approximately 11,000 feet. To accomplish this, we used computer models of the Twin Creek reservoir to predict seismic signatures recorded at the crosswell and 3D seismic scales. Well logs and thin sections of cuttings were integrated with 2D seismic sections to produce petrophysical and geological cross sections, quantify fracture distribution, and determine the petrophysical parameters and reservoir geometries needed for the computer models.

The 2D seismic sections delineated the major boundary surfaces between members of the Twin Creek formation. These boundaries are the tops of the Twin Creek (Giraffe Creek Member), Leeds Creek, Watton Canyon, Boundary Ridge, and Rich. Integration of the surface seismic and well logs demonstrated that the fracture zones cannot be resolved by surface seismic measurements alone. Instead, it is more appropriate to use high-resolution cross-well seismic data. Therefore, we conducted model studies for planning high-resolution interwell seismic experiments to map the fracture zones in the Twin Creek at Lodgepole field.

The modeling results show that seismic waves transmitted between two wells will propagate in fractured carbonate reservoirs at a distance of up to 2,400 feet. Using wall-lock velocity detectors spaced $1.5 \mathrm{~m}$ apart and existing broadband seismic sources, the signal can be received above the noise level. In addition, the large velocity contrast between the main fracture zone and the underlying Boundary Ridge Member suggests that it may be possible to map variations in the geometry of the fracture zone with borehole reflection imaging and traveltime tomography. Finally, the trapped energy and normal modes that are excited in the waveguides will be useful to determine the degree of continuity of permeability barriers, layer thickness variations, inhomogeneities, and boundary surfaces.

\section{B. Geology}

Lodgepole field is the southernmost in a series of oilfields in the Overthrust Belt of southwestern Wyoming and neighboring Utah producing from the Jurassic Twin Creek Formation. Union Pacific Resources (UPRC) has three fields in the play in Summit County, Utah: Lodgepole, Elkhorn, and Pineview. These fields are shown in Figure 1. 
Lodgepole has produced 1,132 MBO. It is a depletion drive reservoir, but most of the wells have significant water production. Six horizontal and twelve vertical boreholes have been drilled in the field (see Figure 2). In particular, note the horizontal well path for the Judd 34-1H and seismic line CREA-25k. Both are used in the integration analysis.

Lodgepole was selected as the type field for this study because of continued horizontal drilling activity in the field and an extensive database:

a. Over 202D seismic lines are available. The lines are both parallel and perpendicular to the fracture orientation.

b. UPRC conducted an outcrop study of the fracture orientation in the Twin Creek formation.

c. Resistivity, density, neutron, sonic, caliper, and gamma ray logs are available for almost all the vertical wells. As an example Figure 3 shows a suite of logs from vertical well 34-2.

d. MWD gamma ray logs are available for every horizontal well and three formation microscanner (FMS) logs have been run $(4-2 \mathrm{H}, 34-1 \mathrm{H}$, and $35-2 \mathrm{H})$.

e. Cuttings are available for the horizontal wells.

C. Stratigraphy

The Twin Creek Formation is approximately 1,500 feet thick in Lodgepole Field and is divided into seven members. The following brief description of each member of the Twin Creek Formation is taken from Bruce (1988):

Gypsum Springs. Approximately 50 feet of sabkha evaporites, red beds, and minor carbonates. It serves as a detachment surface between the Nugget and Twin Creek in the thrusting process. It also created a barrier to hydrocarbon migration between the Nugget and Twin Creek Formations in most places.

Sliderock. Approximately 90 feet of micritic limestone with thin beds of oolitic grainstone and thin shaly zones. No primary porosity has been preserved, but calcite-filled fractures are present.

Rich. Approximately 250 feet of argillaceous limestone that cleans upward. The upper 20 to 50 feet has some intergranular porosity and may be dolomitized. The Rich was the target zone in the 34-1H Judd (30301-01) in Section 34.

Boundary Ridge. Approximately 50 feet of red siltstones and claystones with a sabkha character. It serves as a good marker bed in the Twin Creek Formation.

Watton Canyon. Approximately 250 feet of limestone with thin, tightly cemented oolitic zones. The limestones of the Watton Canyon are thinner bedded and more terrigenous than the Rich Member. An interval approximately 20 feet thick near the base of the Watton 
Canyon is the primary target for most of UPRC's horizontal wells, including the $34-1 \mathrm{H} \mathrm{Judd}$ in Section 34.

Leeds Creek. Approximately 300 feet of interbedded argillaceous and relatively clean limestones. A highly radioactive unit near the top of the member is a devitrified tuff.

Giraffe Creek. Approximately 450 feet of micritic and oolitic carbonates. It becomes sandy toward the top.

The Basal Preuss Silt, a 30 to 100 foot interval of tightly cemented quartz siltstone, overlies the Twin Creek Formation. The Preuss Salt overlies the siltstone. It varies from 20 to 300 feet in thickness.

The Twin Creek Formation overlies the Nugget Formation, which is eolian sandstones. The Nugget is one of the primary hydrocarbon producing reservoirs in the Overthrust Belt.

\section{Study Area}

Lodgepole field extends over six sections (see Figure 2). Section 34 in the center of the field was chosen as the initial study area for this project. The section contains three vertical and two horizontal well.

Structural cross sections were constructed with the logs from the vertical wells in Section 34. The cross sections center on the Watton Canyon and Boundary Ridge Members, which contain the fractured intervals in the Twin Creek Formation. The cross sections include the acoustic velocity, bulk density, gamma ray, caliper, and lithology curves. These cross-sections are given in Figures 6, 7, and 8 .

\section{E. Geologic Controls on Fracture Distribution}

In order to understand the nature of the fracturing in the Twin Creek, thin sections of cutting samples were made in the Judd $34-1 \mathrm{H}$ from 10,400 to $13,470^{\prime}$ and in the Judd 34-1H Redrill from 11,200-11,460' and 12,780-13,180'. These thin sections were impregnated with blue epoxy for porosity and open fracture identification. Selected thin sections were stained with Alizarin Red $\mathrm{S}$ for dolomite and calcite identification.

Only scattered traces of porosity were found in the thin sections, but fracturing was common although selectively controlled by lithology and facies. The significant fracturing was mainly confined to dolomitized mudstones where silt and sand were minor, see Figure 9. The favorable facies for fracturing was a backbank low energy brackish environment (based on the absence of pellets of fossils).

Appendix 1 and 2 summarize the sample descriptions. Appendices 3 and 4 are more detailed descriptions of the data in tabular form. All depths in these appendices are sample depths. 


\section{UPRC JUDD 34-1 H}

The Twin Creek Giraffe Member contains two distinct units, an upper siltstone dominated unit and a lower pellet packstone dominated unit. Clastics are dominate in the Twin Creek Giraffe member from 10,440 to. 10,600', while mudstone and fracturing are rare. The clastics are composed of argillaceous to calcareous pelletal siltstones with minor amounts of sandstones and mudstones. Only traces of vuggy, moldic intercrystalline and microporosity are present from 10,460 to 10,610 '. Only traces of fractures are found in the samples from this interval.

The lower part of this member consists of interbedded pelletal siltstones and silty pellet packstones with significant silty mudstones. The base of this member is marked by increasing amounts of interbedded mudstones. Fractures are very rare in the Giraffe Creek.

The Leeds Creek Member, picked at 10,921', can be divided into an upper oolitic to pellet packstone dominated facies and a lower slightly silty to slightly dolomitic mudstone (top at $11,180^{\prime}$ ). The mudstones at 11,180' are slightly silty and slightly dolomitic with increased fracturing. The fracture interval occurs between 11,180' and into the top of the Watton Canyon Member. An increase in fracturing to $5 \%$ occurs at 11,190 to $11,200^{\prime}$ in slightly to very dolomitic mudstone. Fracturing was very rare in the upper part of the Leeds member, increasing at the base of the Leeds Creek where dolomitization and mudstone content increase.

The Watton Canyon Member (top at 11,260') differs little from the base of the Leeds member. The upper part of the Watton Canyon is predominately dolomitic mudstone. Overall, the Watton Canyon is dominated by dolomitic mudstones with scattered zones of pellet packstone. The dolomitic mudstone rocks are the main host rock for the fracturing, Figure 9. The Watton Canyon mudstones range from slightly to very silty and slightly to very dolomitic. Some scattered slightly calcareous dolomite are interbedded with the very dolomitic mudstones. An increase in dolomitization and decrease in siltyness corresponds to an increase in fracturing. Where interbedded silty pellet packstones predominate, fracturing is rare to absent, Figure 10. Good fractured intervals in the Watton Canyon occur at $11,180-11,360^{\prime}, 11,400-11,470^{\prime}, 11,730-11,900^{\prime}, 11,990-12,540^{\prime}$, $12,960-13,050^{\prime}$, and 13,260 - 13,470' ( sample depths ).

The fracturing in the Watton Canyon is recognized on the basis of healed fractures in the mudstone (5 to $20 \%$ ) and a corresponding occurrence of free crystalline calcite (trace to $20 \%$ ). Most healed fractures show very little open porosity. The open fracture porosity may be represented by the free crystalline calcite. One Watton Canyon interval $\left(11,510\right.$ to $\left.11,610^{\prime}\right)$ contains 5 to $30 \%$ free crystalline calcite and only traces of healed fractures. The top of this interval is pellet packstone dominated while the base of the interval is mudstone dominated. This interval is the only packstone interval with fracturing.

Figure 11 shows a correlation between fracturing and the mudstone facies. In the figure mudstones are present in the intervals where pellets and ooids are absent. 
The fractured intervals (using sample depths) in the Watton Canyon are as follows:

TABLE I

\begin{tabular}{|l|l|}
\hline $11,180-11,360$ & tr $-5 \%$ healed fract. \\
\hline $11,400-11,470$ & tr $-1 \%$ calcite \\
\hline & tr $-20 \%$ healed fract. \\
\hline $11,730-11,900$ & tr $-5 \%$ calcite \\
\hline & $5-10 \%$ healed fract. \\
\hline $11,990-12,540$ & $5-10 \%$ calcite \\
\hline & $5-20 \%$ healed fract. \\
\hline $12,960-13,050$ & $5-15 \%$ calcite \\
\hline & tr $-10 \%$ healed fract. \\
\hline $13,260-13,470$ & $1-5 \%$ calcite \\
\hline & tr $-10 \%$ healed fract. \\
\hline & tr $-10 \%$ calcite \\
\hline & trace of porosity in calcite \\
\hline & trace of dolomite crystals \\
\hline
\end{tabular}

\section{UPRC JUDD 34-1 H Redrill}

Thin sections of cutting samples were made on the base of the Leeds Creek, Watton Canyon, and Rich Members of the UPRC Judd 34-1H redrill from 11,200 to $11,460^{\prime}$ and 12,780 to $13,180^{\prime}$. Because of lack of returns, no samples were collected from 11,460 to $12,780^{\prime}$.

Only three thin sections of Leeds Creek were available for examination. Thin section 11,200 to $11,220^{\prime}$ contained $30 \%$ argillaceous pellet siltstone with calcareous silty pelletal sandstone to siltstone while the two underlying thin sections contained silty to sandy mudstones. The Leeds Creek contained traces of fracturing in the thin section 11,200 to $11,229^{\prime}$ and increased to $10 \%$ at $11,220^{\prime}$. No porosity was present in these thin sections. Slightly silty to slightly sandy dolomitic mudstones were dominant in the base of the Leeds Creek.

The Watton Canyon was encountered at 11,263' in fractured slightly sandy, very to slightly dolomitic mudstones. The mudstones were well fractured with 10 to $20 \%$ healed fractures and 5 to $20 \%$ free crystalline calcite. Below $11,320^{\prime}$ the fracturing decreased to $5 \%$ healed fractures and traces to $5 \%$ crystalline calcite. Where dolomitic mudstones were interbedded with pellet siltstones to pellet packstones, pellets, silt, and sand content increased in the underlying interval. 
Thin sections of the Rich were made from 12,780 to $13,180^{\prime}$ from cutting samples caught after mud returns were established at 12,780'. From the available Rich samples two units were recognized, an upper pellet dominated packstone, and a lower ooid dominated packstone. Fracturing was rare to absent in both units and only occurred at 12,900 to $12,920^{\prime}$ and 12,960 to $12,980^{\prime}$ in the pellet dominated unit where mudstones were present. A zone of free crystalline calcite was observed at 12,920 to $12,980^{\prime}$, which may indicate a fractured interval in the base of the interbedded pellet and mudstone unit. No fracturing was found below $12,980^{\prime}$ or in the ooid dominated packstone.

\section{F. Geologic Controls on Hydrocarbon Production}

Virtually all the effective porosity in the Twin Creek Formation is from fractures. Existing vertical production comes from five members of the Twin Creek. However, horizontal wells produce from fractured intervals in only the Rich and Watton Canyon members. In the Rich the upper 30 feet is the fractured interval.

The Watton Canyon is the most prolific hydrocarbon-producing member of the Twin Creek and it is also the most intensely fractured. The bottom 100 feet of the Watton Canyon contains fractures. A zone approximately 20 feet thick near the base of the Watton Canyon is the primary target for most of UPRC's horizontal wells, including the 34-1H in Section 34. Based on outcrop studies, FMS logs, and production data, the fractures run NW-SE. Fracturing was produced by faulting and tight folding of the hanging wall of the Absaroka Thrust sheet (Bruce, 1988). The Gypsum Springs Member served as a detachment surface between the Nugget and Twin Creek in the thrusting process. The trapping mechanisms is structural closure on asymmetrical anticlines in the hanging wall. The geometry of the Watton Canyon member of the Twin Creek Formation is given by the structural map and the structural cross-section shown in Figures 4 and 5 . Figure 8 is an enlargement of the fractured intervals with the lithology added.

\section{G. Petrophysical Analysis}

Lithologic calculations from the logs, shown in Figure 8, confirm the mixture of lithologies seen in the cuttings and show that fracturing in the Rich Member is in the dolomitic facies. A similar pattern is seen in the Watton Canyon fractured interval in the 34-3 well.

Grading of the fractures on the FMS log, using UPRC's classification, shows two main fracture intervals along the horizontal path of the $34-1 \mathrm{H}$ well: $11,800-12,000^{\prime}$ and 12,300 $13,200^{\prime}$, see Figure 12. The fractured interval from 12,300-13,200' shows up as anomaly on the velocity inversion of the migrated seismic section as shown in Figure 13. Figure 14 shows an example of the FMS log over a fractured interval within the velocity inversion anomaly. In contrast, Figure 15 is the FMS log of an interval outside the velocity inversion anomaly that has little fracturing.

In the fractured interval within the Watton Canyon, sonic velocity ranges from 18,500 20,000 feet/second and bulk density ranges from 2.6 to $2.75 \mathrm{~g} / \mathrm{cm}^{3}$. The upper boundary does not appear to have a significant change in sonic velocity, but the lower boundary is a shale with a sonic velocity of 16,000-17,000 feet/second. Table II summarizes the petrophysical properties of the fractured intervals and the intervals that surround them. 
For most of the fractured interval in the Rich, sonic velocities range from 18,800 to $21,000 \mathrm{feet} / \mathrm{second}$ and bulk density ranges from 2.65 to $2.8 \mathrm{~g} / \mathrm{cm}^{3}$. The interval is bounded above by a shale with a sonic velocity of 16,000 feet/second and below by a shaly limestone with a velocity of approximately 18,000 feet/second. The fractured interval is relatively clean (i.e. shale-free). The upper 15 feet of the fractured interval contains a significant percent of dolomite, which may explain, at least in part, the higher sonic velocities. The fractured interval in the Rich has more shale than the fractured interval in the Watton Canyon.

\section{H. Integration of Surface Seismic and Well Log Data}

Seismic line CREA-25K was selected because it follows the horizontal path of well 34-1H Judd. This well and well 34-2 are plotted in the migrated seismic section given in Figure 16. A synthetic seismogram was produced using the compressional wave velocity log and the density $\log$ from well 34-2. The synthetic was superimposed on the migrated seismic line together with the geological boundaries defined by the well logs in Figure 9. Both Figures 16 and 17 show the horizontal path of well 34-1H intercepting the fracture zone in the Watton Canyon (refer to the crosssection in Figure 8). The Watton Canyon is the most prominent geologic unit in the seismic sections of Figures 16 and 17. Its upper and lower boundaries correlate with the sonic, density and gamma logs, and the seismic events on the seismic sections. In fact, the synthetic seismogram fits reasonably well at the position of the Judd 34-1H in " time-depth" on the seismic section. For example, the top of the Watton Canyon is at $1600 \mathrm{~ms}$ and the top of the Rich Member is at $1640 \mathrm{~ms}$.

In general, the petrophysical boundaries defined by the well logs correlate with the seismic events in the seismic section. The seismic signatures associated with the Watton Canyon suggest lateral velocity changes between wells $34-1 \mathrm{H}$ and $34-2$. There is a change on the seismic signature associated with this region of interest between the trace at position 2190 and well $34-1 \mathrm{H}$. This event may be associated with the petrophysical characteristics of the Rich Member in the region below the Watton Canyon

The fractured zones are located in the bottom part of the Watton Canyon Member and in the top of the Rich Member, where these fractured zones are intercepted by well 34-2. Both fractured zones are associated with low gamma ray counts. The fracture zone in the Watton Canyon is separated from the fracture zone in the Rich by the 50-foot thick Boundary Ridge Member, which consists of siltstones and claystones. A reflection event associated with this boundary is observed in the seismic sections of Figures 16 and 17 and is below the horizontal path of well $34-1 \mathrm{H}$. This analysis suggests that the Watton Canyon in the region between both wells under consideration is associated with the "trough"- reflection response , which may be caused by the presence of a fractured zone containing vertical fractures. This fractured zone is intercepted by the horizontal well in the bottom part of the Watton Canyon. The FMS logs recorded in the horizontal well intercepted vertical fractures having a variable fracture density distribution. 


\section{Interwell Seismic Modeling \\ Computer Models}

To evaluate transmission and detection of seismic waves in the Twin Creek Formation of the Utah-Wyoming Overthrust Belt, computer models were constructed using rock physical properties from the Lodgepole field. We have selected petrophysical parameters from a cross-section including the Watton Canyon, Boundary Ridge, and Rich Members between wells 34-2 and 34-3. The P-wave and S-wave velocities, densities, and thicknesses from these two wells are given in Table II. These parameters were used to construct a twelve-layer model to produce particle velocity and pressure seismograms to evaluate if the seismic energy in the formations will reach the desired interwell distance of about $1 / 2$ mile in Lodgepole field. The P-wave and S-wave velocities of the twelve-layer model are shown in Figure 18.

The seismic responses were calculated using a Ricker wavelet as a source pulse function for a pressure source placed at $31.5 \mathrm{~m}$ and $55 \mathrm{~m}$ below the top layer interface of the Watton Canyon Member and an array of 60 detectors placed at $800 \mathrm{~m}$ from the source well. Synthetic seismograms and spectral density plots of interwell seismic signatures were produced by taking into account the source signal strength of $10 \mathrm{~cm}^{3}$ and receiver noise levels using a buried point source mechanism [i.e, a point source in a fluid-filled borehole can be represented by an equivalent force system of a monopole and a dipole ( Parra et al., 1995, and Parra et al., 1993, )].

The spectral density plots and amplitude-depth distribution curves were produced to characterize interwell seismic signatures and to evaluate the propagation characteristics of seismic waves in the Twin Creek Formation for planning interwell seismic experiments.

\section{Synthetic Seismograms and Amplitude Depth Distribution Curves for a Source at $31.5 \mathrm{~m}$ Below Top Layer Interface}

Full waveform particle velocity seismograms given in Figures 19 and 20 show strong $P$ headwave events and reflections. The vertical component particle velocity captures most of the main boundaries such as the Watton Canyon fracture member/ heterogenous zone, and the shale I lower fracture interval. The heterogenous zone and the shale unit are part of the Boundary Ridge Member, where trapped waves are observed in the vertical particle velocity seismogram. On the other hand in the horizontal particle velocity and the pressure seismograms (given in Figure 20) it is difficult to correlate the reflections with the formation interfaces. However, amplitude-depth distribution curves of the horizontal component show a better correlation with the layer interfaces in Figure 21, in particular with the contact between the main fracture interval in the Watton Canyon Member and the heterogenous zone (formed by units having different rock physical properties) above the shale unit. These plots also show that most of the energy appears to be distributed in the main fracture interval at higher frequencies. This suggests that a source having a center frequency of about $400-500 \mathrm{~Hz}$ will be appropriate to resolve the main features in the Watton Canyon formation at the well separation of $800 \mathrm{~m}$. 


\section{Spectral Density for a Source at 31.5 m Below Top Layer Interface}

We have used computer modeling to analyze how far the source signal can travel in realistic media and still be received above the noise level at a desired interwell distance. The models were used to produce spectral densities of interwell seismic signatures by taking into account the source signal strength and receiver noise levels (see Figures 22 and 23). Seismic traces were selected for the pressure source at $31.5 \mathrm{~m}$ and detectors at $31.5 \mathrm{~m}$ and $22.5 \mathrm{~m}$ (both source and detectors are placed below top layer interface) to produce spectral density plots. These plots were compared with ambient seismic noise levels having RMS values of $1 \mathrm{mPa}$ and $100 \mathrm{mPa}$, respectively. The computed spectral density of vertical and horizontal particle velocity components are above the ambient seismic noise level in quiet and noisy environments. The spectral amplitude of the horizontal component is about 20-25 dB above the specified velocity noise level of $7.1 \mu \mathrm{cm} / \mathrm{s}$, which was based on the RMS pressure noise level of $100 \mathrm{mPa}$, and the spectral amplitude of the vertical component is about $12.5 \mathrm{~dB}$ above the same velocity noise level. Alternatively, the spectral density signatures of the pressure are below the specified ambient seismic noise level of $1 \mathrm{mPa}$ and the electronic noise level of the OAS hydrophone.

\section{Synthetic Seismograms and Amplitude Depth Distribution Curves for a Source at $55 \mathrm{~m}$ Below Top Layer Interface}

Full waveform synthetic seismograms given in Figures 24 and 25 show direct waves, head waves, trapped waves (leaky modes), reflections, and normal modes (Rayleigh-type of waves) for a source placed in the Boundary Ridge Member at $55 \mathrm{~m}$ below the top layer interface. The horizontal component particle velocity and the pressure seismograms show strong head waves and trapped waves (see Figure 24). In particular, the horizontal component particle velocity has head wave events followed by direct events, reflection, and leaky modes. This seismogram captures the boundary between the main fractured interval (top) and the heterogenous zone (bottom) which is formed by four petrophysical units having different rock physical properties. Also the interface between the shale and lower fractured zone can be detected by measuring the horizontal particle velocity. Similarly, the vertical component seismogram captures the same interfaces (Figure 25). However, it also shows Rayleigh-type of waves (normal modes) associated with the shale waveguide at about the source position of $55 \mathrm{~m}$. The modes are excited in the shale as well as in the heterogeneous zone below the main fracture interval. These results suggest that guided waves can be used to map the continuity of shales which are permeability barriers in the Twin Creek reservoir.

In addition, amplitude-depth distribution curves of the horizontal and vertical particle velocities were produced to evaluate the propagation frequency for resolving the features of interest in the formation at the well separation of $800 \mathrm{~m}$, when a source is placed in the low-velocity shale. A center frequency of $200 \mathrm{~Hz}$ can excite Rayleigh-type of waves in the shale waveguide as wells as waves in the low-velocity units within the heterogeneous zone (see Figure 26). On the other hand, a center frequency of about $400 \mathrm{~Hz}$ will resolve geological units of about 2-3 m thick. 


\section{Spectral Density for a Source at 55 m Below Top Layer Interface}

In a similar manner as the previous example, seismic traces were selected for the pressure source at $55 \mathrm{~m}$ and detectors at $55.5 \mathrm{~m}$ and $61.5 \mathrm{~m}$ below top layer interface to produce spectral density plots shown in Figures 27 and 28 . These plots were compared with ambient seismic noise levels having RMS values of $1 \mathrm{mPa}$ and $100 \mathrm{mPa}$, respectively. The spectral densities signatures of the pressure are below the specified ambient seismic noise level of $1 \mathrm{mPa}$ and the electronic noise level of the OAS hydrophone. Alternatively, the computed spectral density of vertical and horizontal particle velocity components are above the ambient seismic noise level in quiet and noisy environments. The spectral amplitude of the horizontal component is about $25 \mathrm{~dB}$ above the specified velocity noise level of $6.1 \mu \mathrm{cm} / \mathrm{s}$, which was based on the RMS pressure noise level of $100 \mathrm{mPa}$, and the spectral amplitude of the vertical component is about $20 \mathrm{~dB}$ above the noise level of $100 \mathrm{mPa}$. In this case, when the source is placed in the waveguide the spectral density is greater than when the source is placed in the main fracture interval of the Watton Canyon. The main reason is that energy can be trapped in the waveguide and it can travel for long distances in the low velocity zone. On the other hand the energy in high-velocity formations will leak as the wave propagates between wells at large well separations.

\section{DISCUSSIONS AND CONCLUSIONS}

Correlation between the lithology and the well logs and the migrated seismic data from the Lodgepole field served to identify seismic events associated with geological units of interest. The surface seismic delineates the major geological boundaries between members of the Twin Creek Formation. The fracture zones were not resolved directly by surface measurements techniques. The surface seismic section shows a reflection at about $1640 \mathrm{~ms}$ (at the bottom of the Watton Canyon) that was interpreted as a boundary surface between the Watton Canyon Member and the Rich Member. In this case, the fracture zones (in the Watton Canyon and the Rich Members) and the Boundary Ridge Member were interpreted to be part of this boundary surface. On the other hand, a velocity anomaly observed in the inversion plot correlates with the fractured density distribution which was determined from the FMS log.

In order to resolve the petrophysical units at the scale of the fracture zones and the Boundary Ridge Member, cross-well seismic measurements are more appropriate. As a result, we have conducted a model study for planning high-resolution interwell seismic experiments to map or delineate the fracture zones of interest in Lodgepole field. The models demonstrate that a source having a center frequency of $400-500 \mathrm{~Hz}$ can resolve the features of interest in Lodgepole between wells $800 \mathrm{~m}$ apart. We expect that if we can transmit energy in the frequency range of $1000 \mathrm{~Hz}$ we have the potential to map the fracture zones in the Watton Canyon and in the top of the Rich Member, as well as to properly identify the boundaries that were not resolve by the surface seismic. The surface seismic did not resolve the fracture zones and the heterogeneous Boundary Ridge Member, which is formed by four units with different petrophysical properties and a low-velocity shale with a thickness of approximately $9 \mathrm{~m}$.

The computer model study suggests that high-resolution cross-well measurements are necessary to map the fracture zones in Lodgepole field, in particular in the Watton Canyon Member 
and the upper part of the Rich Member. The model results also show that it is feasible to detect seismic waves propagating in the Twin Creek Formation above the ambient noise levels of a noisy environment using particle velocity detectors and existing broadband seismic sources at well separations exceeding $800 \mathrm{~m}$. In addition, the spacing between detectors should be about $1.5 \mathrm{~m}$ and a source frequency range about $1000 \mathrm{~Hz}$. We expect that the travel time tomography and the reflection imaging can be used to map the fracture boundary zones. The trapped energy and normal modes excited in waveguides can be useful information to determine the degree of continuity of zones of interest such as permeability barriers, layer thickness variations, inhomogeneities, and boundary surfaces. Polarization diagrams from three-component seismic data can be used to evaluate the anisotropy associated with the presence of vertical fractures in the formations. 
TABLE \|

PETROPHYSICAL PROPERTIES OF SELECTED INTERVALS IN THE TWIN CREEK FORMATION, LODGEPOLE FIELD, SUMMIT COUNTY, UTAH

Interval

Thickness
(feet)

Vc Range
(feet/second)

Vc Average
(feet/second)

pb Average
$\left(\mathrm{g} / \mathrm{cm}^{3}\right)$

pbRange

Watton Canyon

Above fractured interval

100

$17,000 *-19,700$

18,800

2.7

$2.6-2.8$

Shale $^{*}$ above the fractured interval $\quad 10$

$17,500-18,500$

18,200

2.68

2.6-2.7

Upper part of fractured interval

$19,000-20,000$

19,300

2.69

2.6-2.7

Main part of fractured interval

60

$18,700-20,000$

19,300

2.7

2.6-2.78

Below fractured interval

$$
1 \text { shale }
$$

10

$15,800-18,500$

17,500

2.68

2.6-2.7

$\stackrel{\leftarrow}{\circ}$

2

10

19,000

2.75

\section{Boundary Ridge Member}

1 shale

\section{2}

3 shale (above fractured interval)

\section{Rich Member}

$\begin{array}{lr}\text { Fractured interval } & 30 \\ \text { Shale* below fractured interval } & 5 \\ \text { Below fractured interval } & 145\end{array}$

10

25
$16,000-19,300$

19,000

16,000

$19,000 *-21,000$

20,000

17,300

18,200
2.8

2.68
2.8 (upper 10 feet)

2.71 (lower 20 feet)

2.75

2.65-2.8

2.71

Data came from a comparison of 3 vertical boreholes (34-1, 34-2, and 34-3). Compressional velocities (Vc) were obtained from sonic logs; bulk densities $(\rho b)$ were obtained from density logs. Sections of the borehole with washouts have reduced density values, and these sections were not factored into the density averages.

* These Ve values are from zones with increased gamma ray counts and are probably shaly. 


\section{References}

Bruce, C.L., 1988, Jurassic Twin Creek Formation: a fractured limestone reservoir in the Overthrust Belt, Wyoming and Utah: Rocky Mountain Association of Geologists, pp. 105-120.

Parra, J.O., Sturdivant, V.R., and Xu, P.-C., 1993, Interwell seismic transmission and reflections through a dipping low-velocity layers, Journal of the Acoustical Society of America, 93, 1954-1969.

Parra, J.O., 1995, A feasibility study of high-resolution reverse VSP and interwell seismic methods for hydrocarbon reservoir characterization: The Log Analysts, May-June, pp. 64-81.

Parra, J.O.,Zook,B.J., and Xu,P.-C., 1995, Transmission and detection of guided seismic waves in attenuating media: model study, paper to be published in Geophysics.

Parra, J.O., Zook, B.J., and Collier, H.A., 1996, Interwell seismic logging for formation continuity at the Gypsy test site, Oklahoma: Journal of Applied Geophysics. v. 35, pp. 45-62.

Parra, J.O., 1996, Characterization of fracture reservoirs using static and dynamic data: from sonic and 3D seismic to permeability distribution: September Quarterly Report, U.S. Department of Energy, Contract No. DE-AC22-94PCp1008; SwRI Project 15-7939.

Svor, T.R., and Meehan, D.N., 1991, Quantifying horizontal well logs in naturally fractured reservoirs: Society of Petroleum engineers, $66^{\text {th }}$ Annual Meeting, Dallas, Texas, 469-480.

\section{LIST OF CAPTIONS}

Figure 1. Regional map showing location of Overthrust Field.

Figure 2. Location map showing horizontal well path for the 35-1H Judd and seismic line CREA-25K

Figure 3. Well log display for well 34-2 Judd including gamma ray, deep lateral log, compressional wave velocity and density-neutron porosity.

Figure 4. Structural map on top of Watton Canyon member of the Twin Creek Formation.

Figure 5. Structural cross-section along traverse AA'.

Figure 6. A structural cross-section through the Wells 34-2 and 34-1 at the Lodgepole field.

Figure 7. A structural cross-section through the wells 34-2 and 34-3 at the Lodgepole field.

Figure 8. Stratigraphic cross-section of Lodgepole Field.

Figure $9 . \quad$ Fractured dolomitic mudstone facies.

Figure 10. Thin sections photomicrographs.

Figure 11. Relationship between fracturing and pellet/peloid/oolite facies in the 34-1H well.

Figure 12. Distribution of gradable fractures from the FMS log in the 34-1H well.

Figure 13. Velocity inversion of migrated seismic section CREA-25K along horizontal well path 34-1H.

Figure 14. FMS image of an interval with high-grade fractures within the inversion velocity anomaly.

Figure 15. FMS image of an interval with low-grade fractures outside of the velocity inversion anomaly.

Figure 16. Migrated seismic section CREA-25k along horizontal well path of well 34-1H Judd.

Figure 17. Migrated seismic section CREA-25k of Figure 8 with synthetic seismogram inserted at well location 34-2 Judd.

Figure 18. Compressional and shear wave velocity parameters for the twelve-layer model.

Figure 19. (a) Common source horizontal particle velocity seismogram with source at a depth of $31.5 \mathrm{~m}$ below top layer interface. Well separation $=800 \mathrm{~m}$; (b) Common source vertical particle velocity seismogram with source at a depth of $31.5 \mathrm{~m}$ below top layer interface. Well separation $=800 \mathrm{~m}$

Figure 20. Common source pressure velocity seismogram with source at a depth of $31.5 \mathrm{~m}$ below top layer interface. Well separation $=800 \mathrm{~m}$.

Figure 21. Amplitude-depth distribution of horizontal particle velocity produced by source at a depth of $31.5 \mathrm{~m}$ below top layer interface. 
Figure 22. (a) Comparison of spectral density of horizontal particle velocity with seismic noise levels in quiet and noisy environments. Source depth $=31.5 \mathrm{~m}$ below top layer interface; (b) Comparison of spectral density of vertical particle velocity with seismic noise levels in quiet and noisy environments. Source depth $=31.5 \mathrm{~m}$ below top layer interface.

Figure 23. Comparison of spectral density of pressure with electronic and seismic noise levels in quiet and noisy environments. Source depth $=31.5 \mathrm{~m}$ below top layer interface.

Figure 24. (a) Common source horizontal particle velocity seismogram with source at a depth of $55 \mathrm{~m}$ below top layer interface. Well separation $=800 \mathrm{~m}$.; (b) Common source pressure velocity seismogram with source at a depth of $55 \mathrm{~m}$ below top layer interface. Well separation $=800 \mathrm{~m}$.

Figure 25. Common source vertical particle velocity seismogram with source at a depth of $55 \mathrm{~m}$ below top layer interface. Well separation $=800 \mathrm{~m}$. (a) $100 \mathrm{~ms}$ time window; (b) $200 \mathrm{~ms}$ time window.

Figure 26. Amplitude-depth distribution of vertical particle velocity produced by source at a depth of $55 \mathrm{~m}$ below top layer interface. (a) Amplitudes of 100 and $200 \mathrm{~Hz}$., (b) Amplitudes of 200 and $400 \mathrm{~Hz}$.

Figure 27. (a) Comparison of spectral density of horizontal particle velocity with seismic noise levels in quiet and noisy environments. Source depth $=55 \mathrm{~m}$ below top layer interface.; (b) Comparison of spectral density of vertical particle velocity with seismic noise levels in quiet and noisy environments. Source depth $=55 \mathrm{~m}$ below top layer interface.

Figure 28. Comparison of spectral density of pressure with electronic and seismic noise levels in quiet and noisy environments. Source depth $=55 \mathrm{~m}$ below top layer interface. 


\section{Appendix 1}

\section{Lithologic Description of the Twin Creek Formation \\ UPRC Judd \# 34-1H}

Giraffe Creek Member (10,440' MD)

$10,400-10,460^{\prime}$

Pelletal siltstones with trace sandstone; some argillaceous; scattered porosity at base.

10,460-10,610 $\quad$ Predominately interbedded pellet siltstones \& pelletal packstones.

10,610-10,930 Interbedded packstones \& mudstones; some interbedded oolitic sandstones \& siltstones: only a trace of fractures in the sample.

\section{Leeds Creek Member (10,918' $\mathrm{MD})$}

Kick-off point 10,972' MD

10,930-11,020' Interbedded pelletal siltstone with minor pellet packstones \& mudstones.

11,020-11,060' Sandy oolitic packstones interbedded with sandstone; traces of interbedded oolitic sandstone; no fracturing.

11,060-11,180 $\quad$ Pelletal siltstones, pelletal packstones with minor interbedded mudstones; trace fracturing.

Watton Canyon Member (11,270' MD)

11,180-11,360 Slightly dolomitic silty mudstones; slight increase in fracturing (trace - 5\%); slight-very dolomitic mudstones; some very silty mudstone units.

$11,360-11,400^{\prime} \quad$ Slightly silty to very silty pelletal packstones to pelletal siltstones; decreasing fracturing (trace or less); increasing siltstones- sandstones.

$11,400-11,470^{\prime} \quad$ slight-very silty mudstone; increasing fracturing (10-20\%); slight increase in dolomite; slight decrease in silt \& siltstones.

11,470-11,550' Silty pelletal packstone with minor scattered mudstones; decrease in dolomite; decrease in fracturing (trace or less).

Top 30' target zone (11,590' MD)

11,550-11,730' Slightly dolomitic mudstone; trace very dolomitic mudstone; decreasing silt; increasing dolomite.

11,730-11,900' Slightly to very dolomitic mudstone; abundant dolomitic and dolomite at top; increasing dolomite; increasing fracturing (trace-10\%); 5-10\% healed fractures; 5-10\% crystal calcite. 
11,900-11,990 Slightly silty, slightly dolomitic mudstone; decrease fractures (1\% to trace); 5 $10 \%$ crystal calcite.

Fault 12,354' MD $\sim 94^{\prime}$ throw

$11,990-12,540^{\prime} \quad$ Very slightly silty, slightly dolomitic mudstone; trace to scattered ooids; increase fracturing (5-20\%); increasingly silty at base; 5-15\% crystal calcite.

12,540-12,690 Slightly dolomitic mudstone; decreasing silt; decreasing dolomite; decreased fracturing (trace); decreasing crystal calcite.

12,690-12,960' Very slightly dolomitic pellet packstone; pellet packstone with abundant pellets; trace fracturing; increasing silt.

12,960-13,050 $\quad$ Slightly dolomitic mudstone with minor interbedding pellet packstone; increasing fractures (trace-10\%); increasing crystal calcite.

13,050-13,260 $\quad$ Silty pellet packstone with minor slightly dolomitic mudstone; increasingly silty, decreased fracturing; slight increase in porosity 13,260-13,290.

\section{Fault 13,330' MD $\sim 9$ ' throw}

$13,260-13,470^{\prime}$

Slightly dolomitic to dolomitic mudstone with traces of dolomite beds; some pellet packstone; decreasing silt; 5-20\% dolomite at 13,260-13,350; increasing fractures (trace-10\%); traces of porosity at top. 
Appendix 2

\section{Lithologic Description of the Twin Creek Formation UPRC Judd \# 34-1H Redrill}

Leeds Creek Member (10,946' MD)

$11,200-11,220^{\prime}$

Silty sandstone-siltstone; $30 \%$ argillaceous pelletal siltstone; trace of fractures.

Watton Canyon Member (11,270' MD)

$11,220-11,460^{\prime} \quad$ Slightly silty to sandy dolomitic mudstone; increased fracturing (5-20\%); decreasing siltstone.

Watton Canyon target (11,436-11,480' MD)

$11,460-11,780^{\prime} \quad$ Missing

Rich target $\left(11, \mathbf{6 4 0}^{\prime} \mathrm{MD}\right)$

$11,780-13,020^{\prime} \quad$ Sandy to silty ooid pellet packstone; decreased fracturing (trace or less).

$13,020-13,180^{\prime} \quad$ End of samples; silty ooid packstone; decreasing fracturing (trace to none). 


\section{OVERTHRUST FIELDS \\ UTAH \& WYOMING}

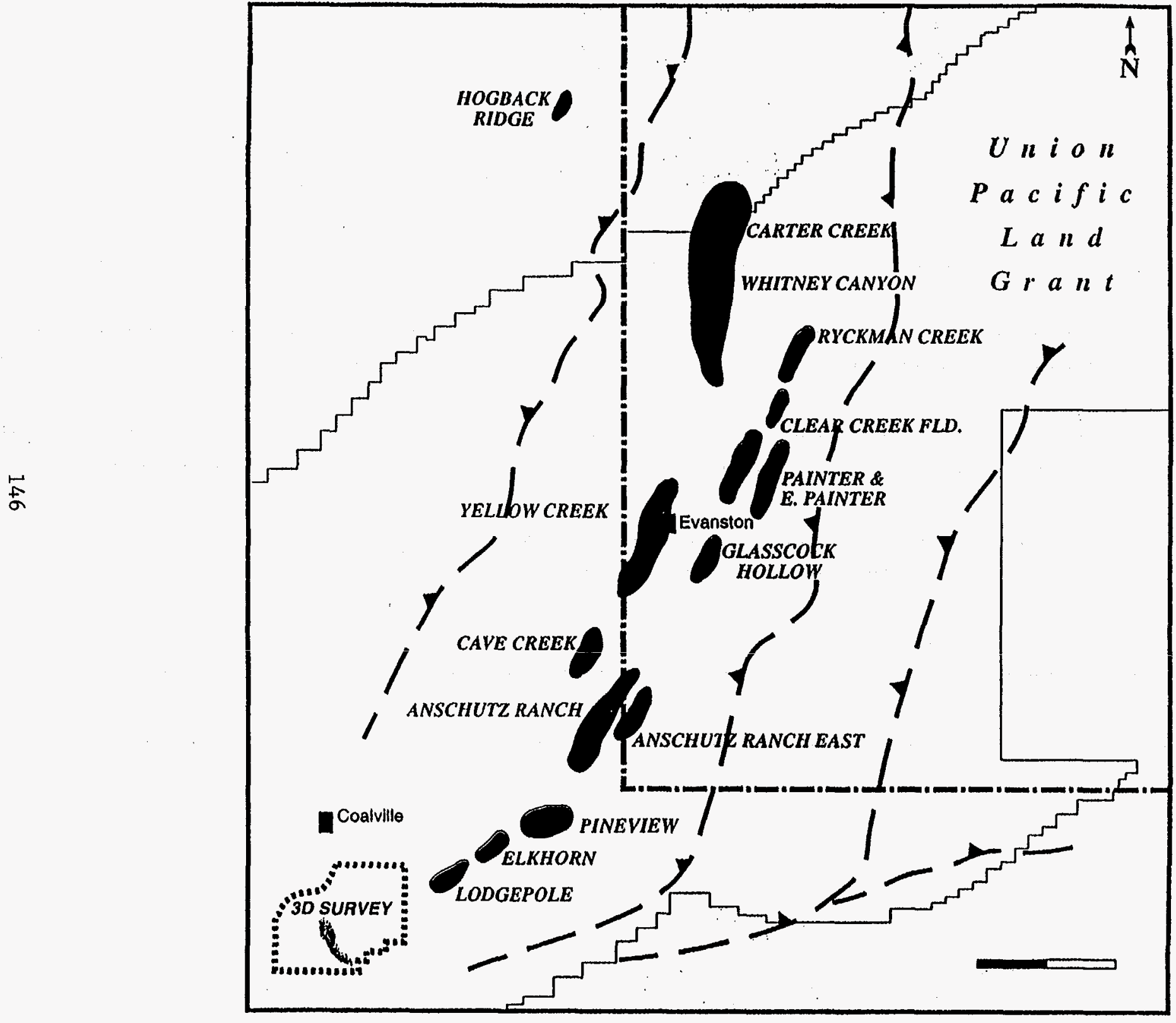




\section{Structure Map on Top of Watton Canyon Member of the Twin Creek Formation}

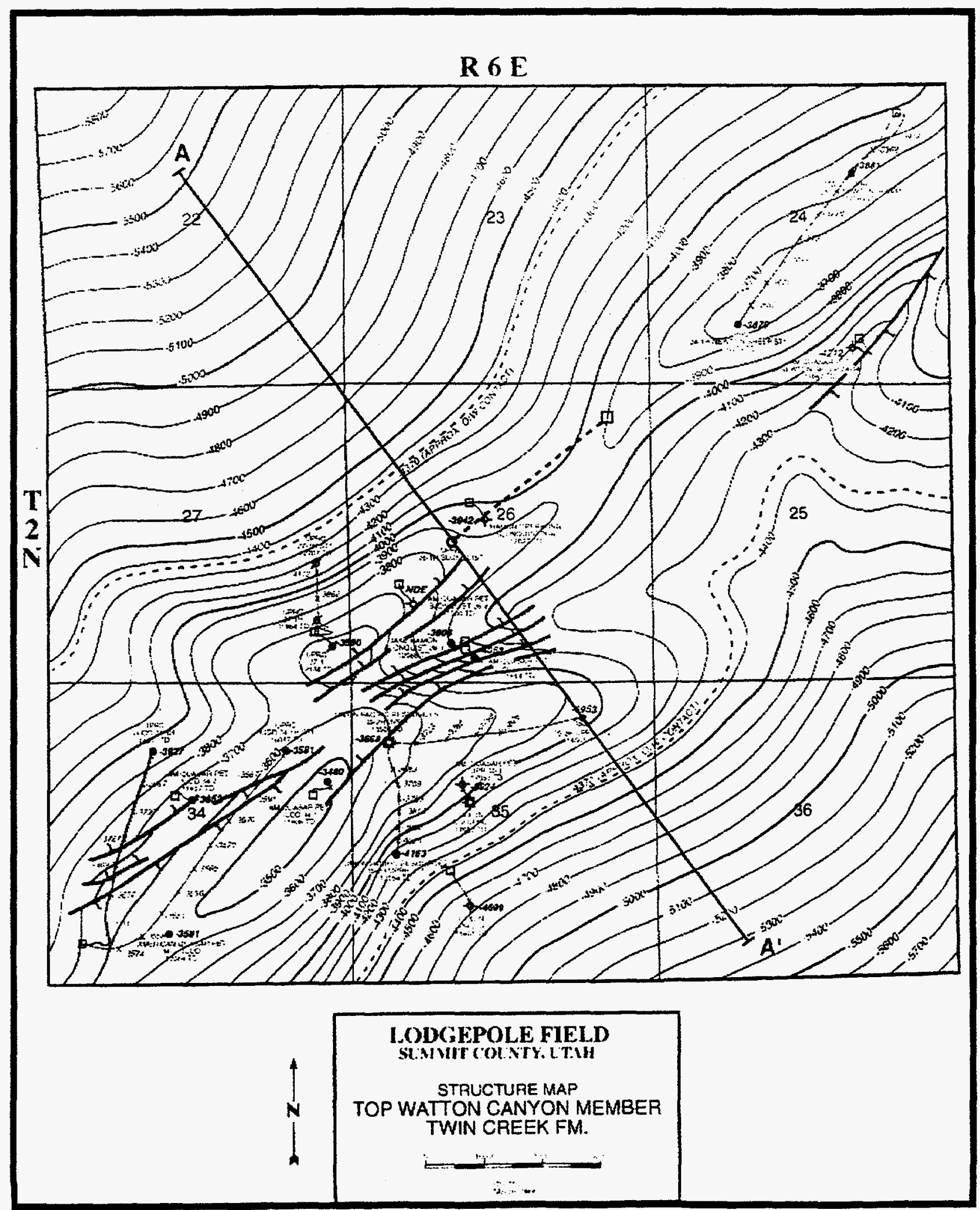




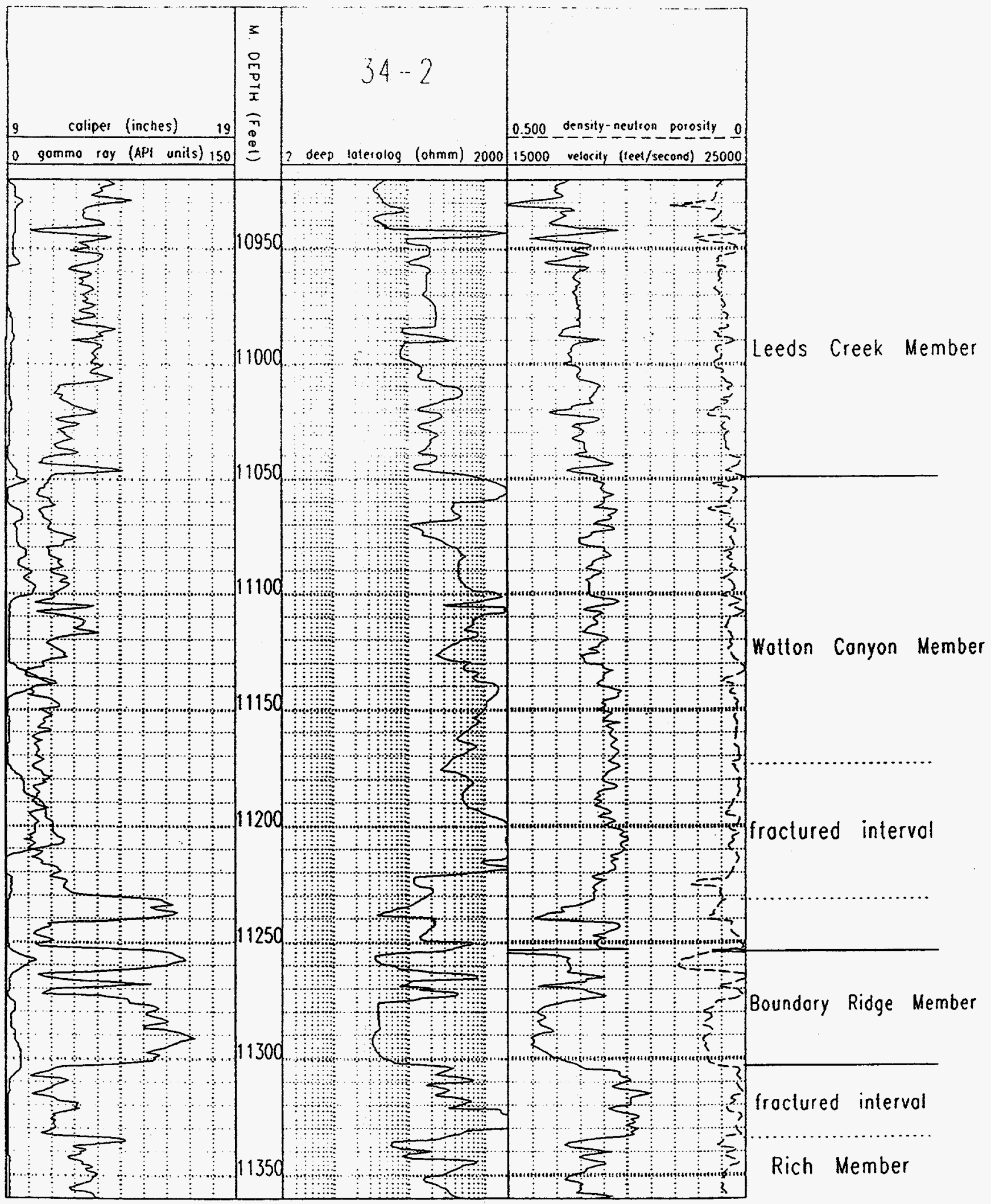

Figure 3. 
Location Map Showing Horizontal Well Path for the 35-1H Judd and Seismic Line CREA-25k

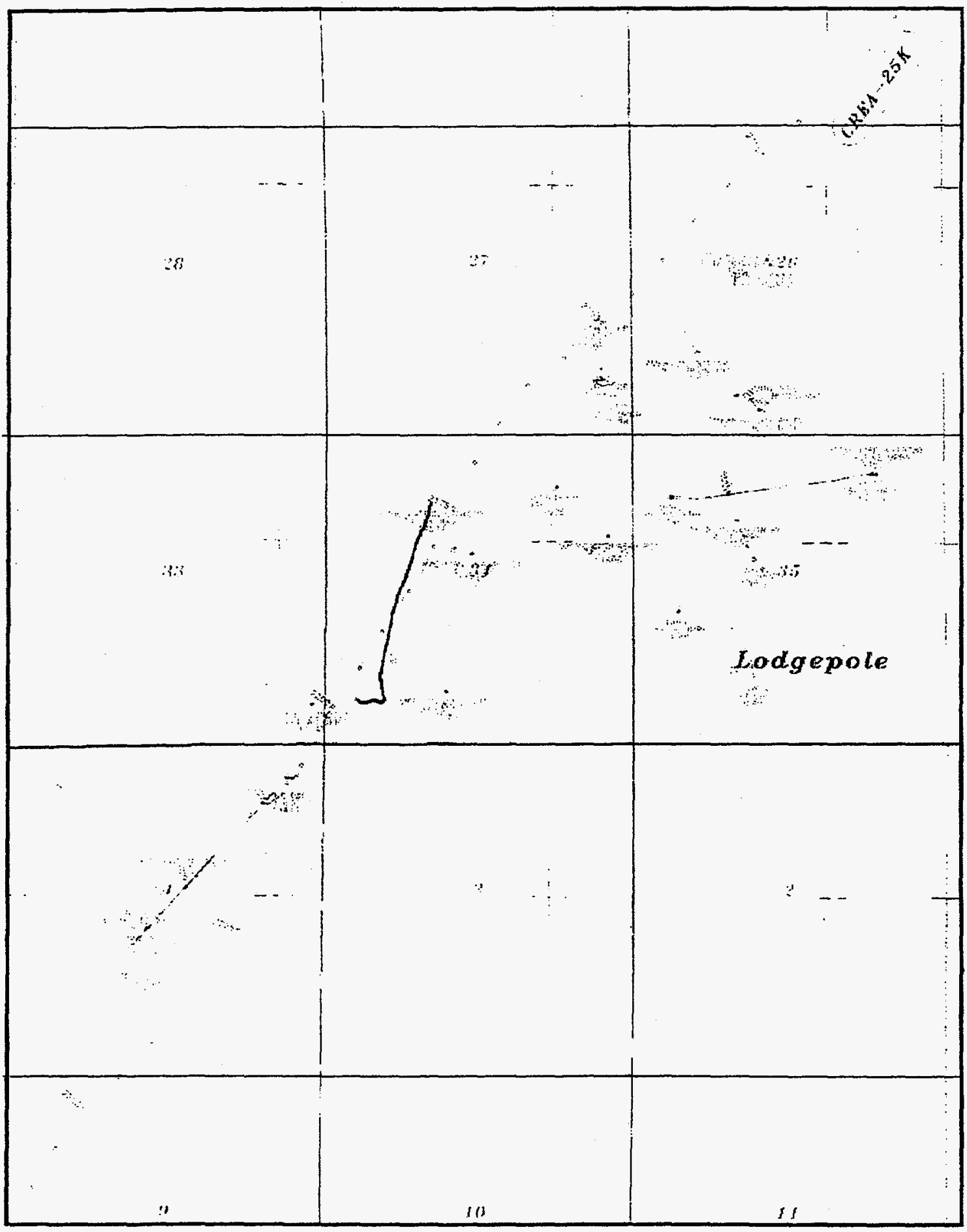

Figure 4 
STRUCTURAL CROSS-SECTION A-A'

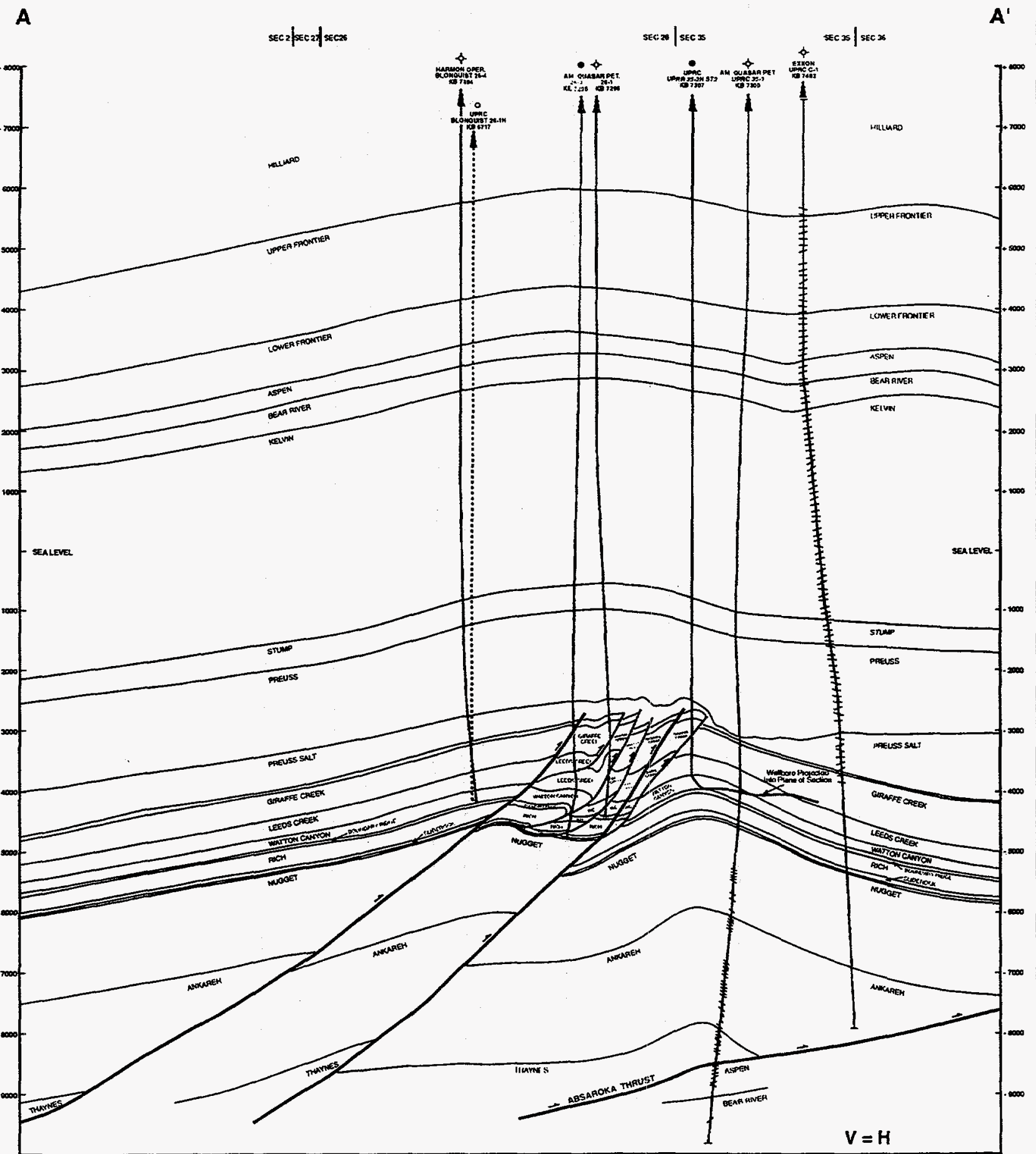

Figure 5

150

LODGEPOLE FIELD

SUMMIT COUNTY, UTAH

STRUCTURAL CROSS-SECTION 




Figure 6. 


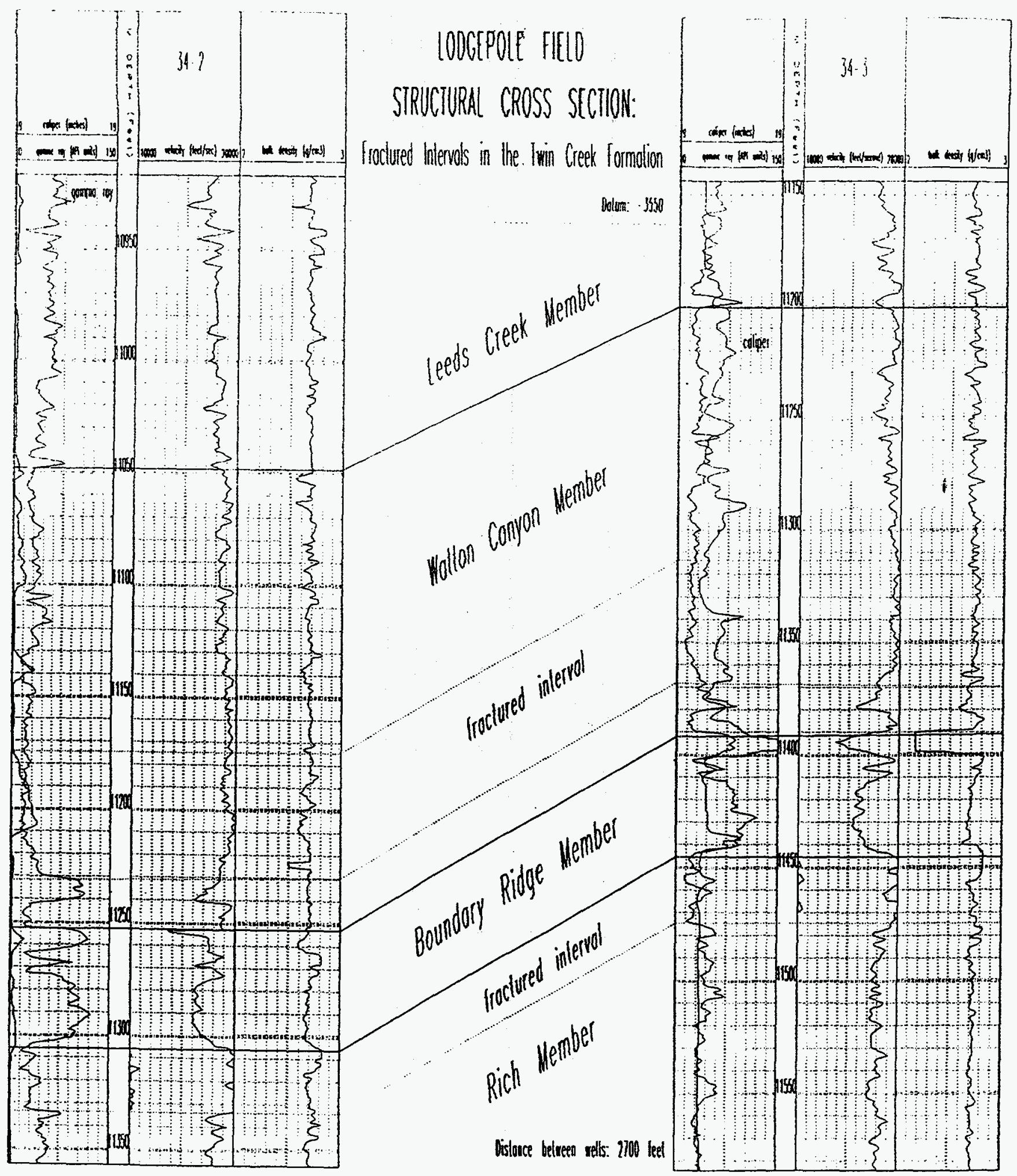

Figure 7 . 


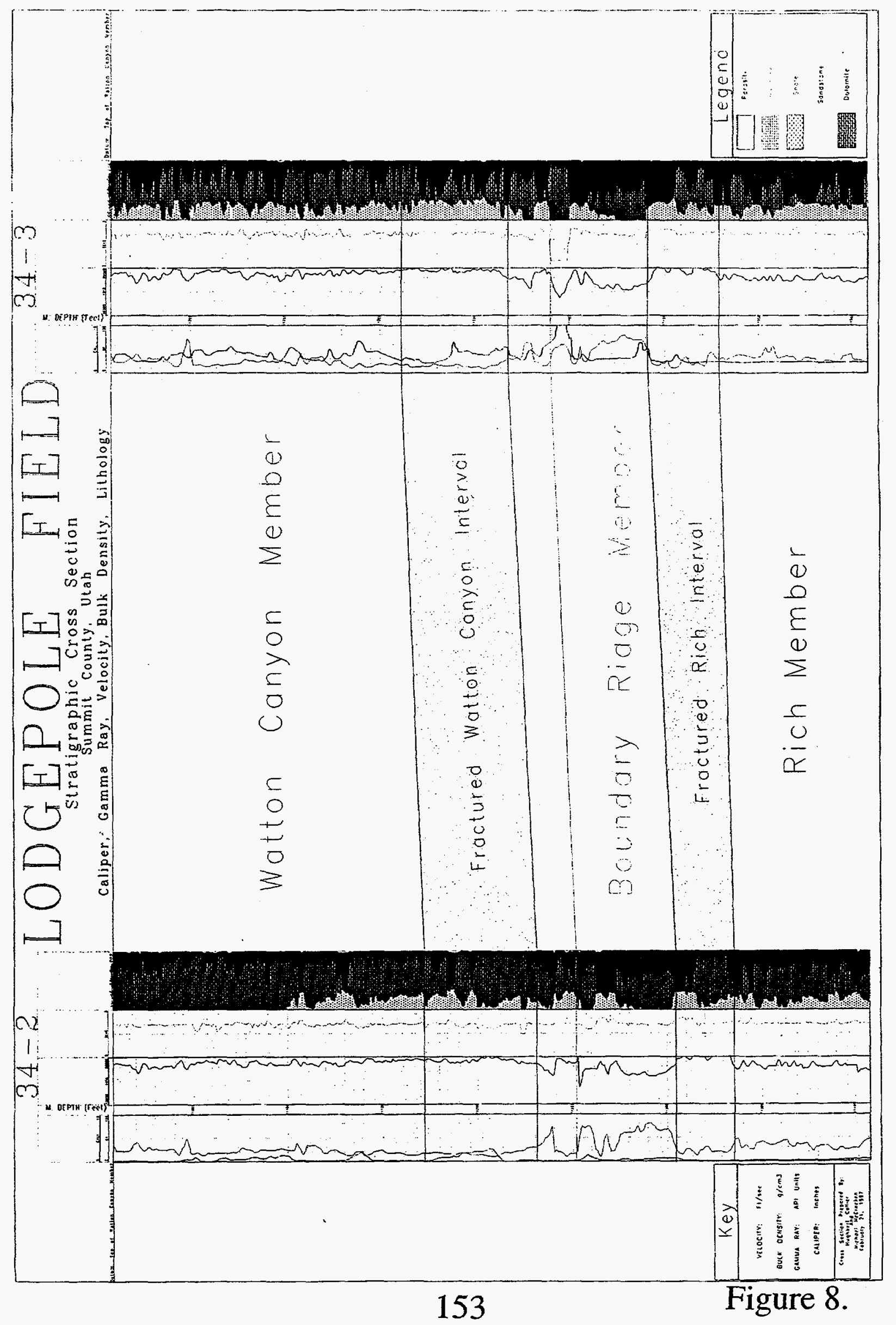



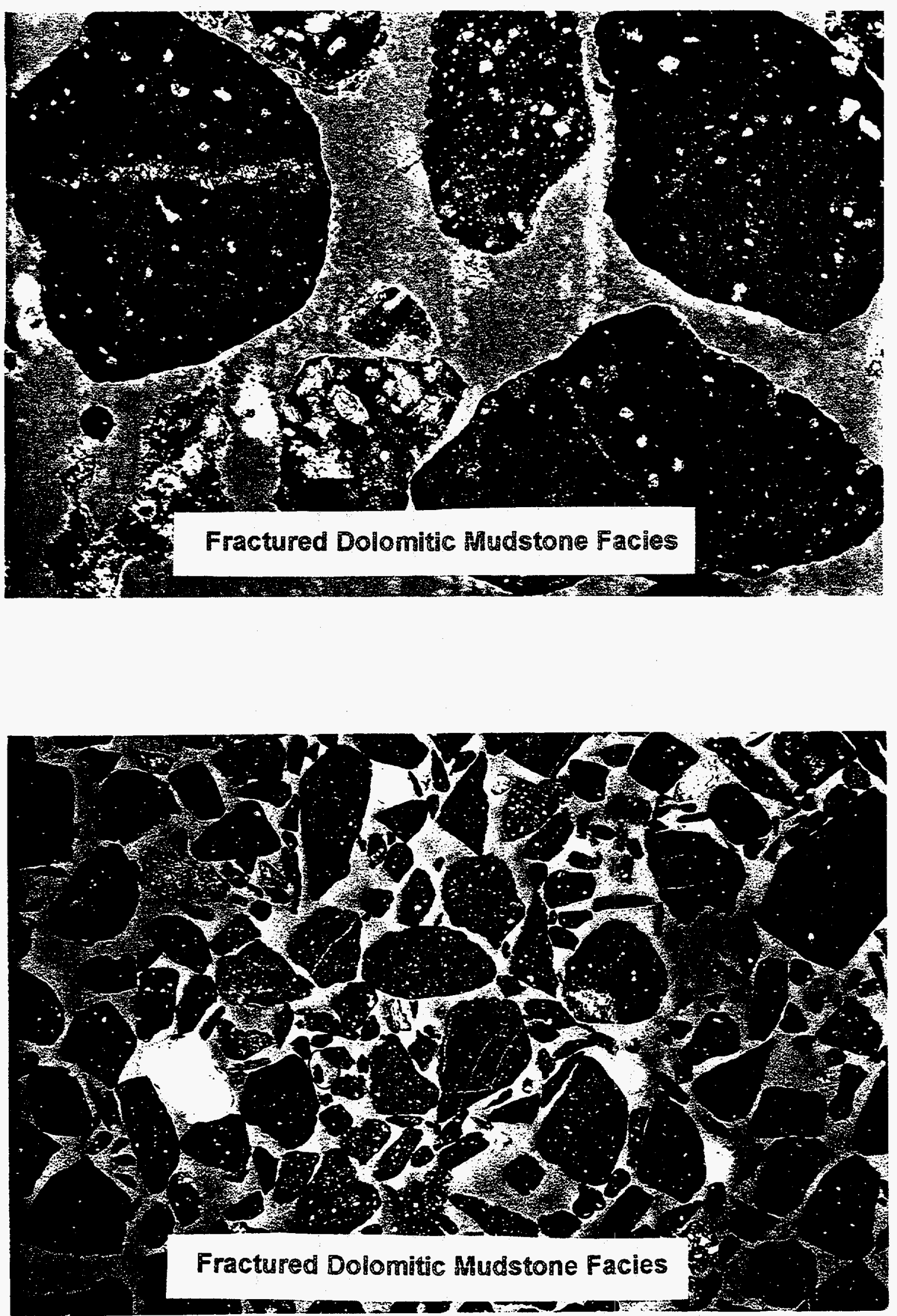

Figure 9 

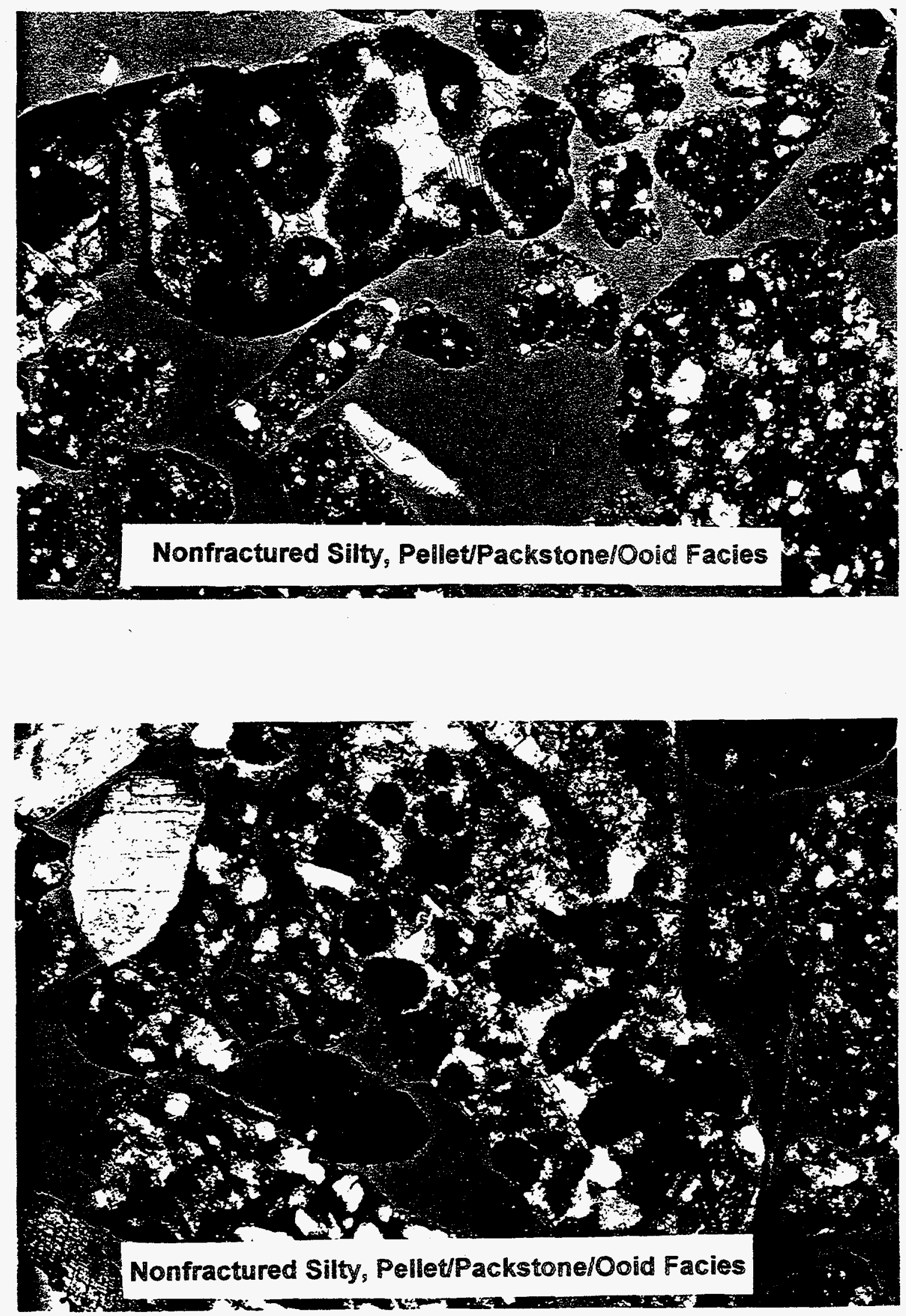

Figure 10 


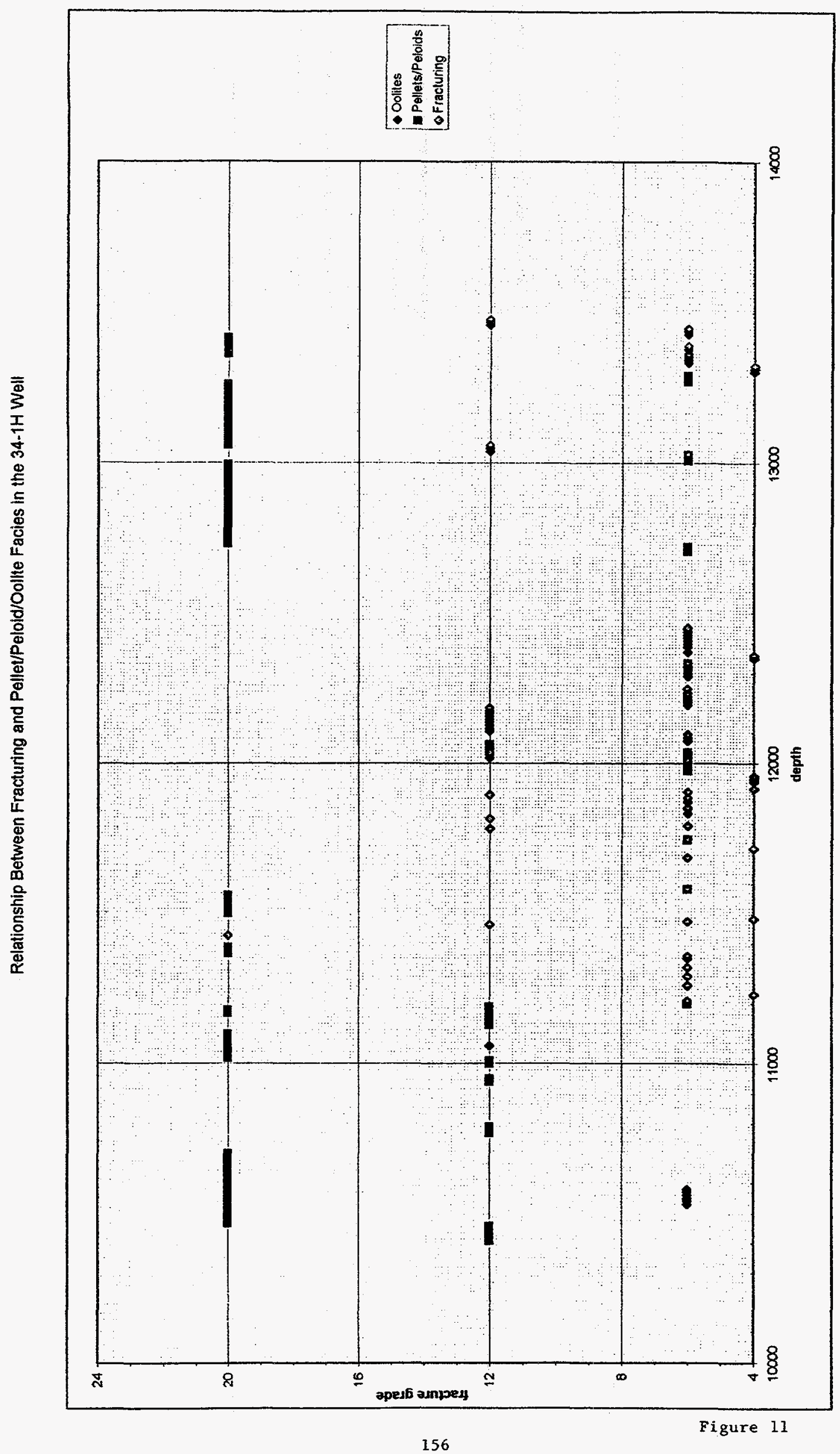




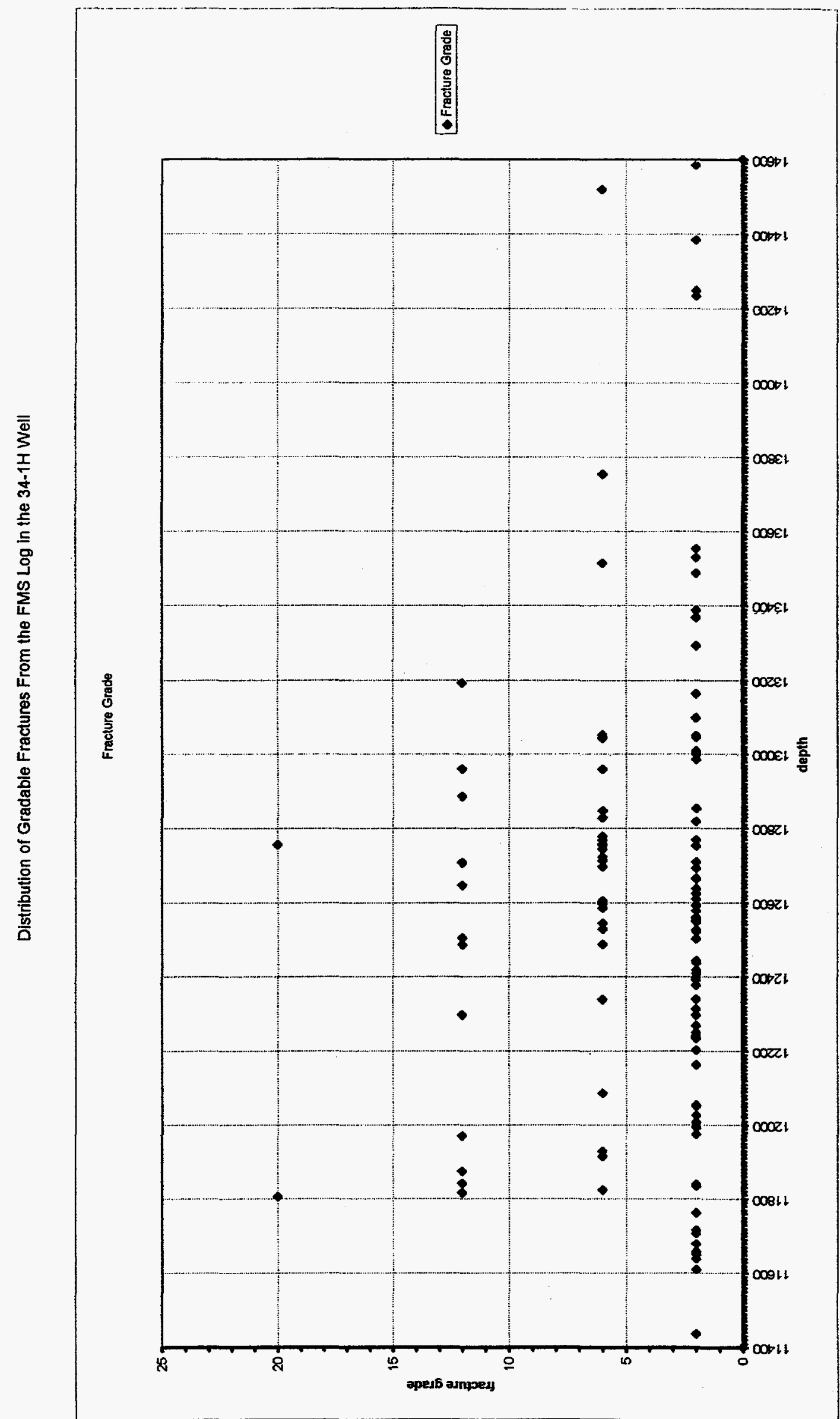


VELOCITY INVERSION OF MIGRATED SEISMIC SECTION CREA-25K ALONG HORIZONTAL WELL PATH 34-1H

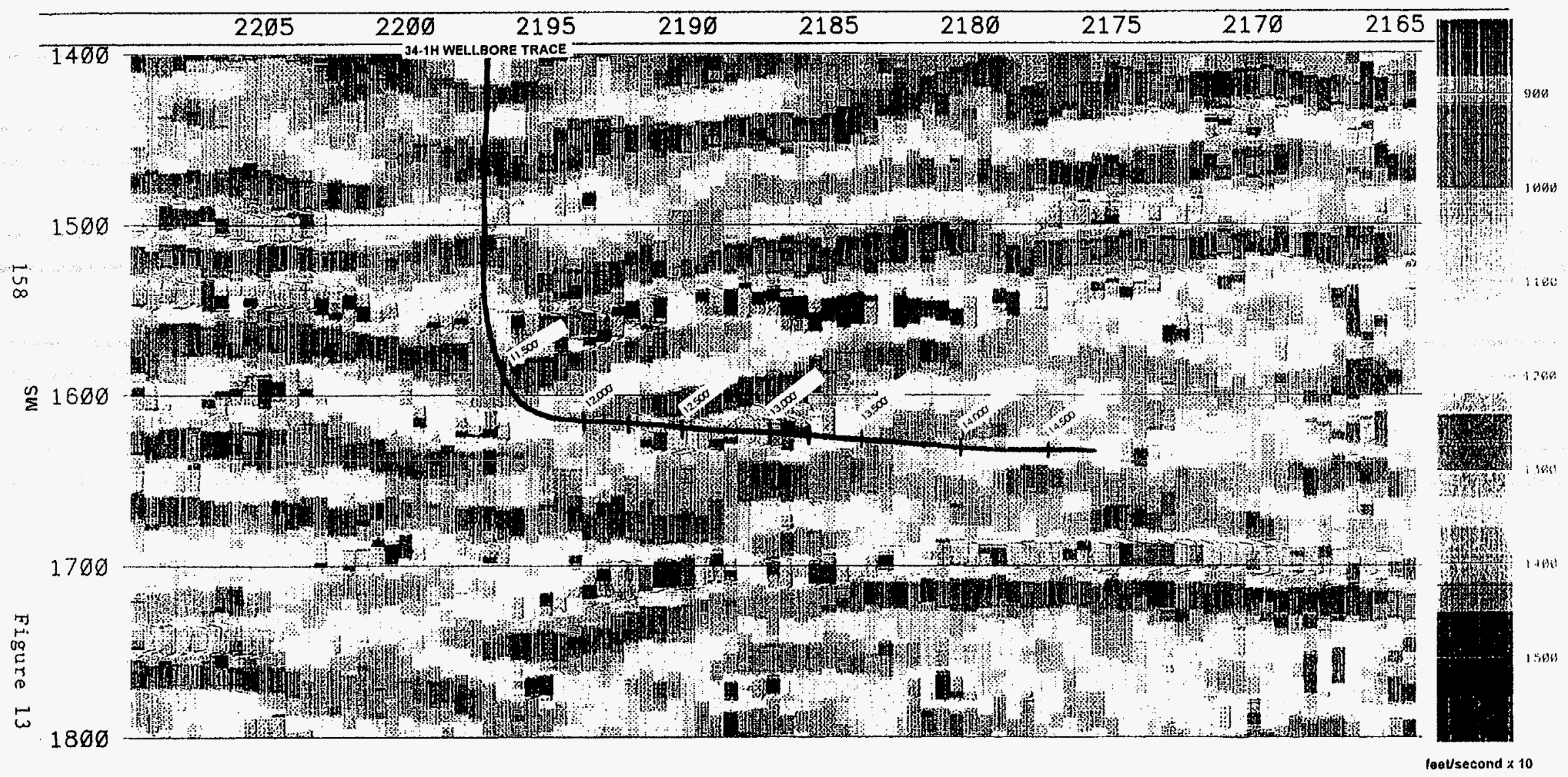




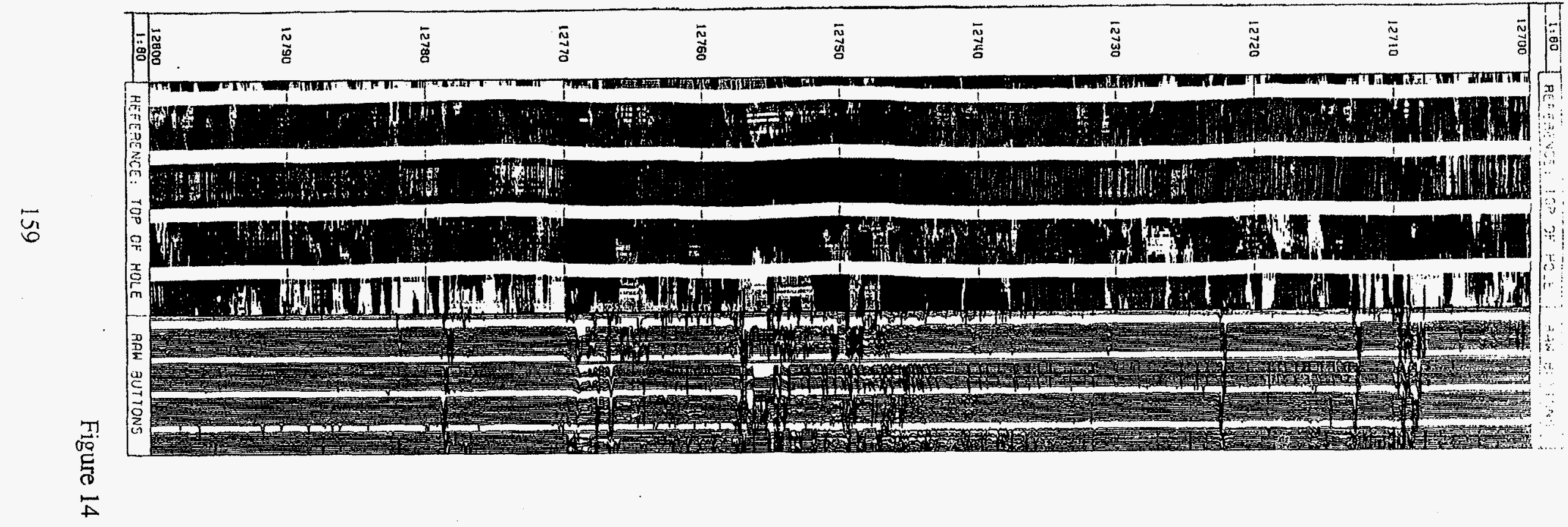




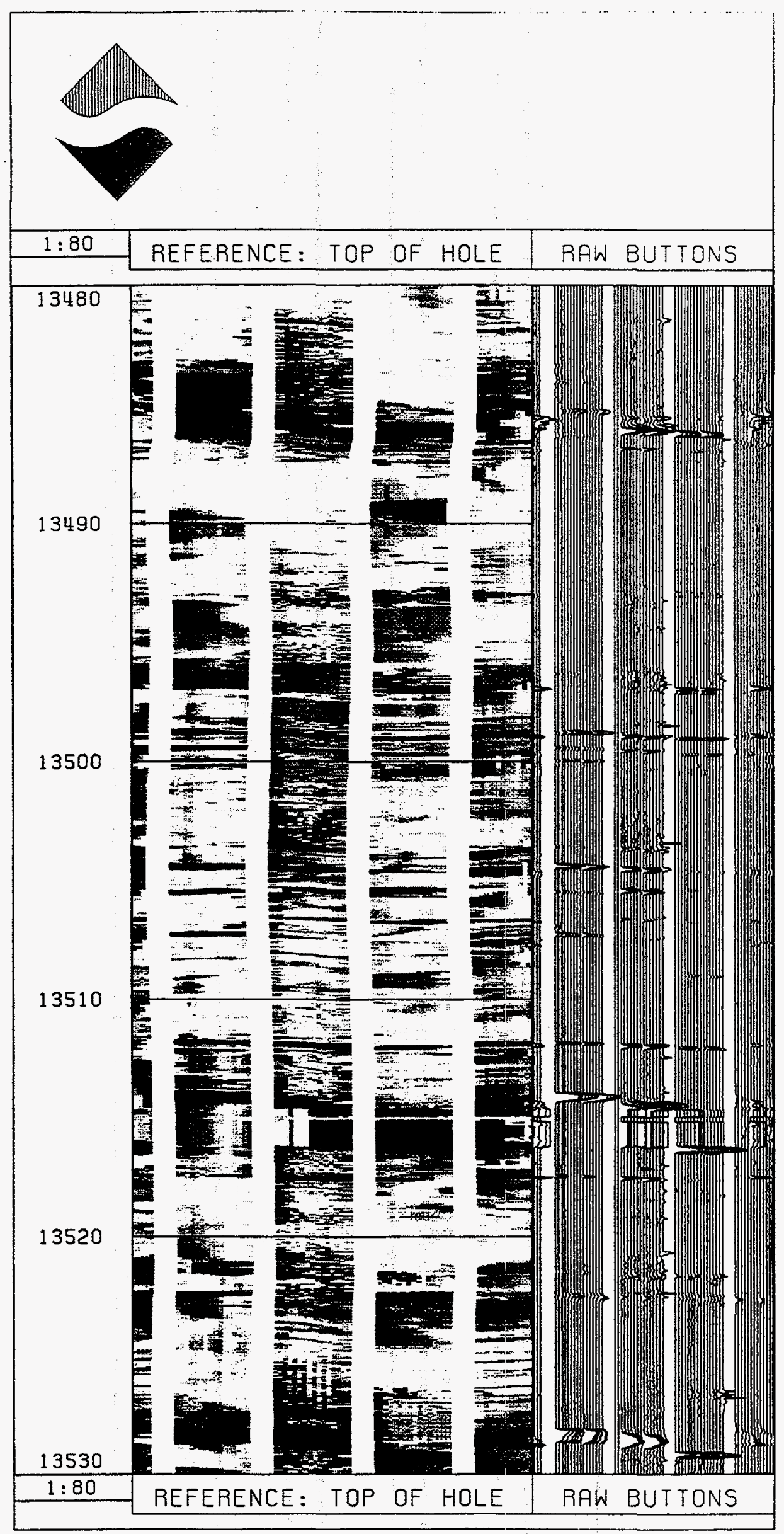

Figure 15 


\section{Migrated Seismic Section CREA-25K Along Horizontal Well Path of Well 34-IH Judd}

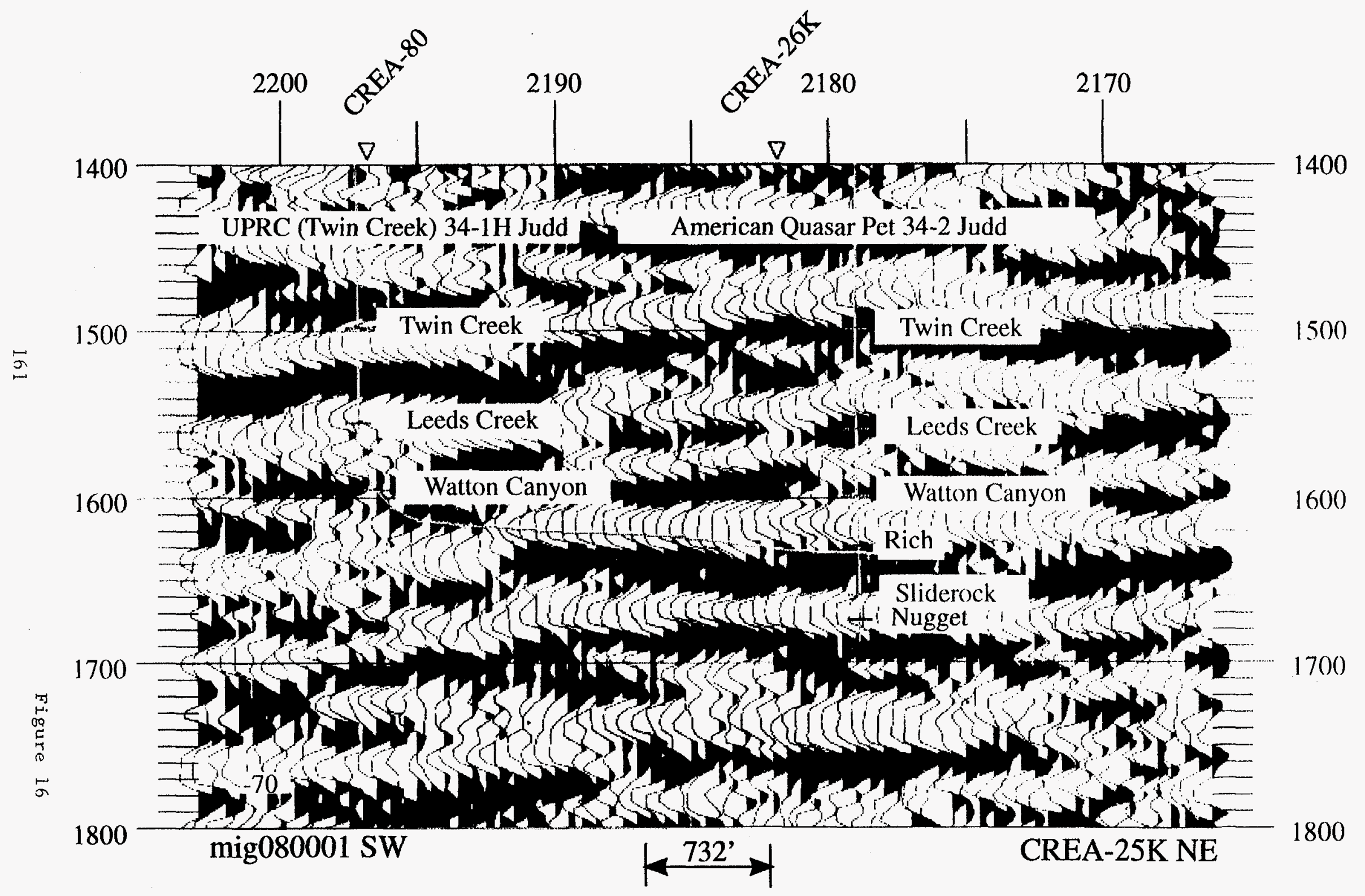




\section{Migrated Seismic Section CREA-25K with Synthetic Seismogram Inserted at Well Location 34-2 Judd}

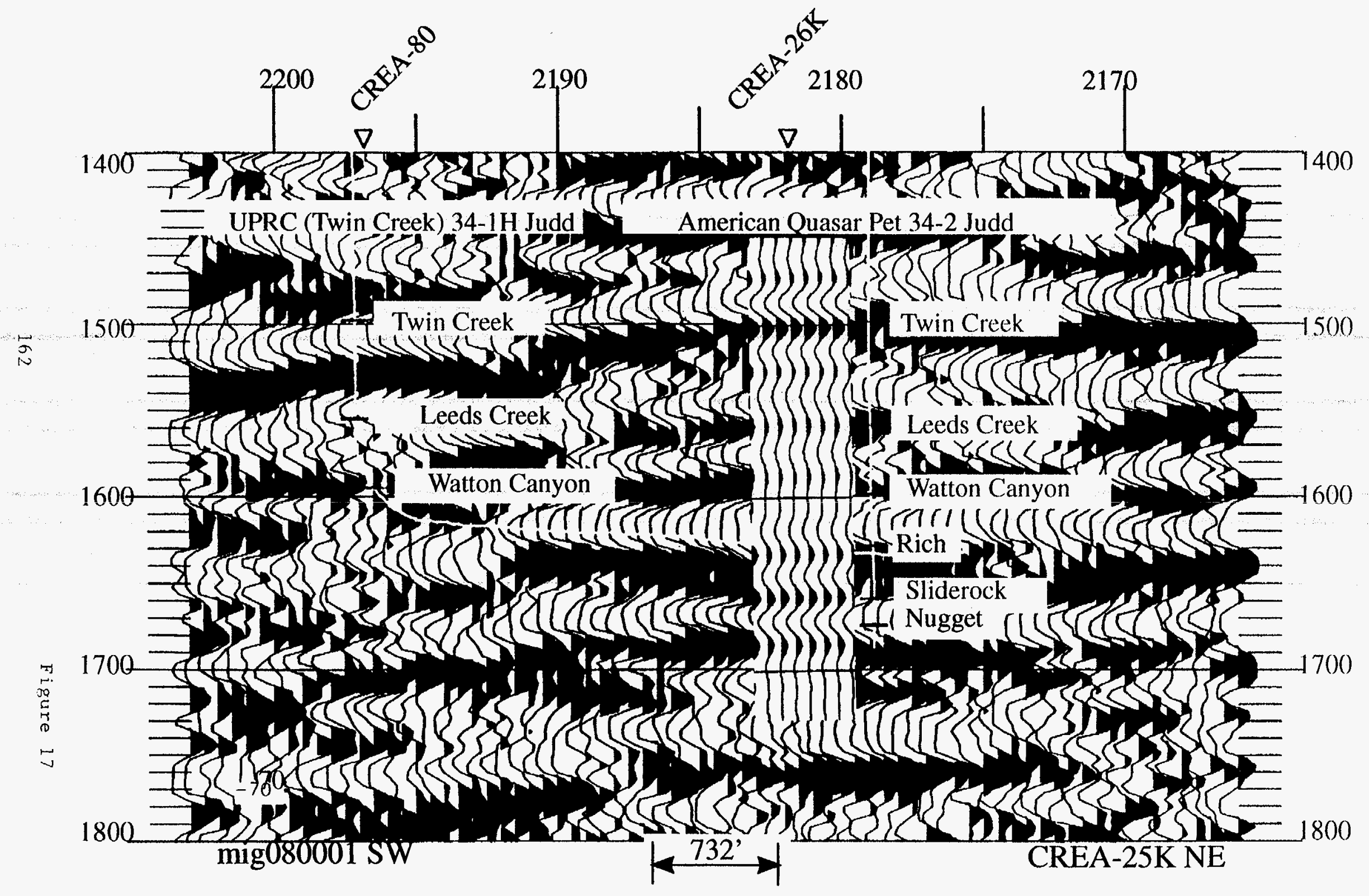




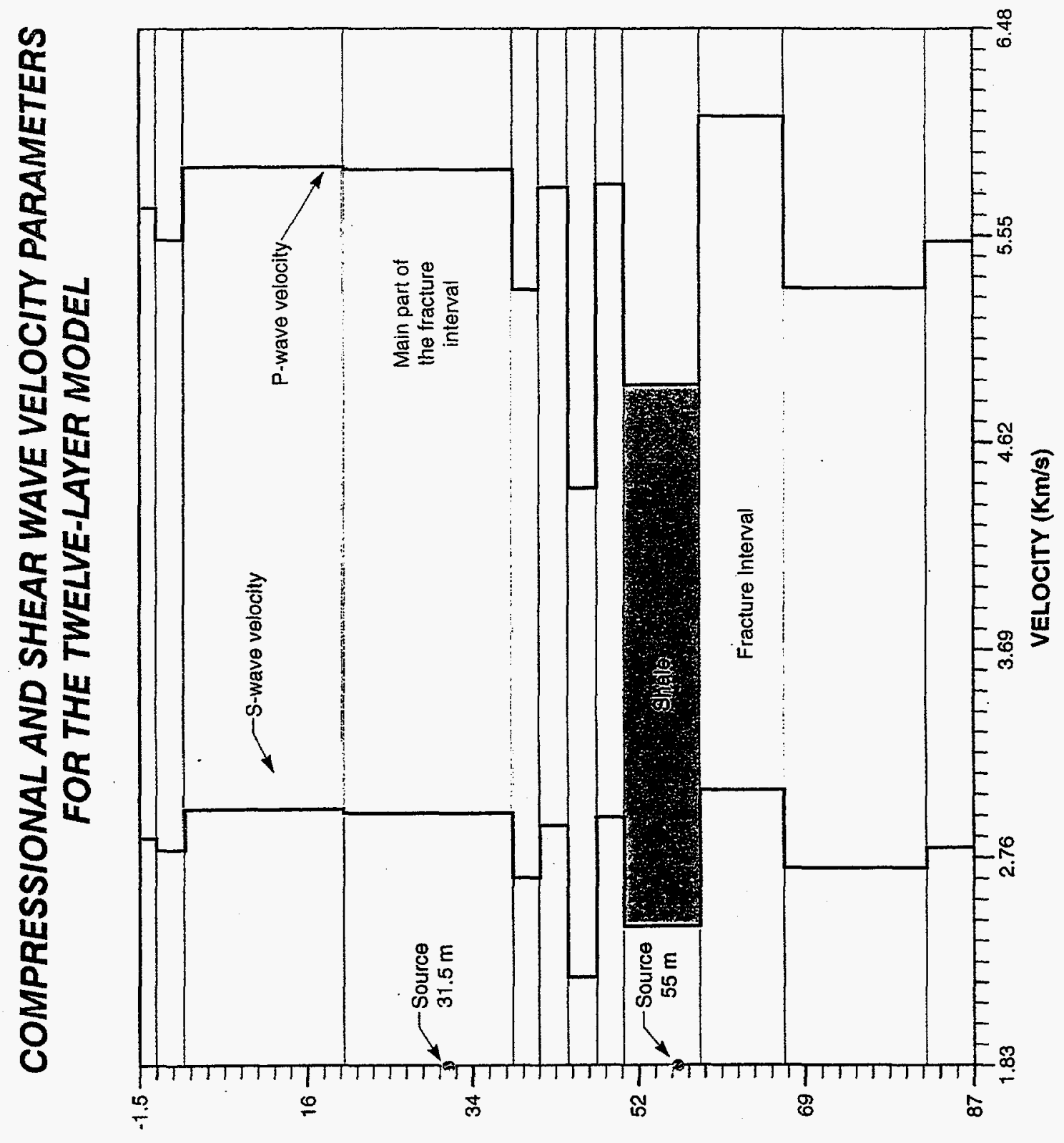

(ui) $H \perp d \exists G$

Figure 18 


\section{COMMON SOURCE PARTICLE VELOCITY SEISMOGRAMS WITH SOURCE AT A DEPTH OF $31.5 \mathrm{~m}$ BELOW TOP LAYER INTERFACE}

Well Separation $=800 \mathrm{~m}$

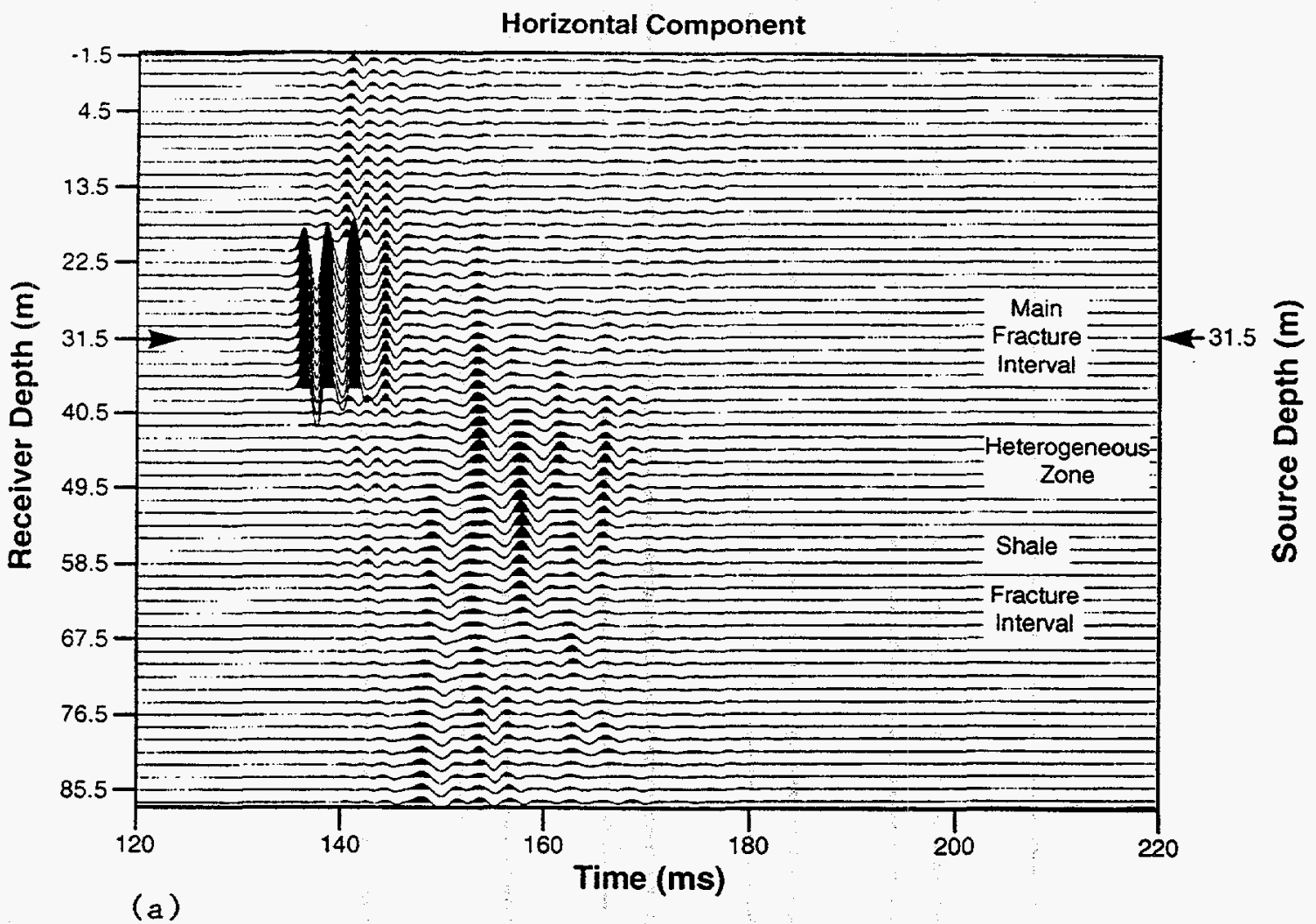

Vertical Component

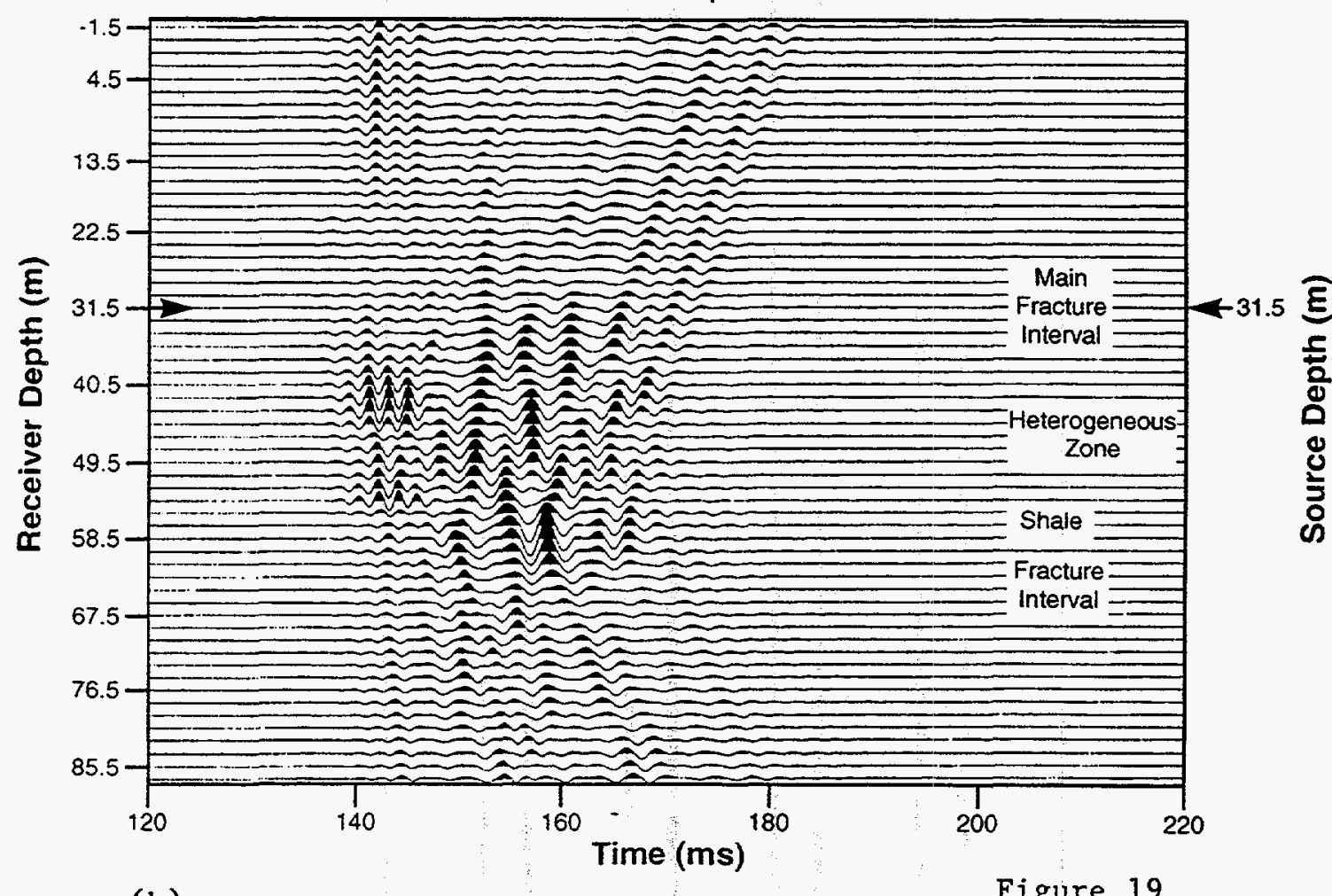

(b) 


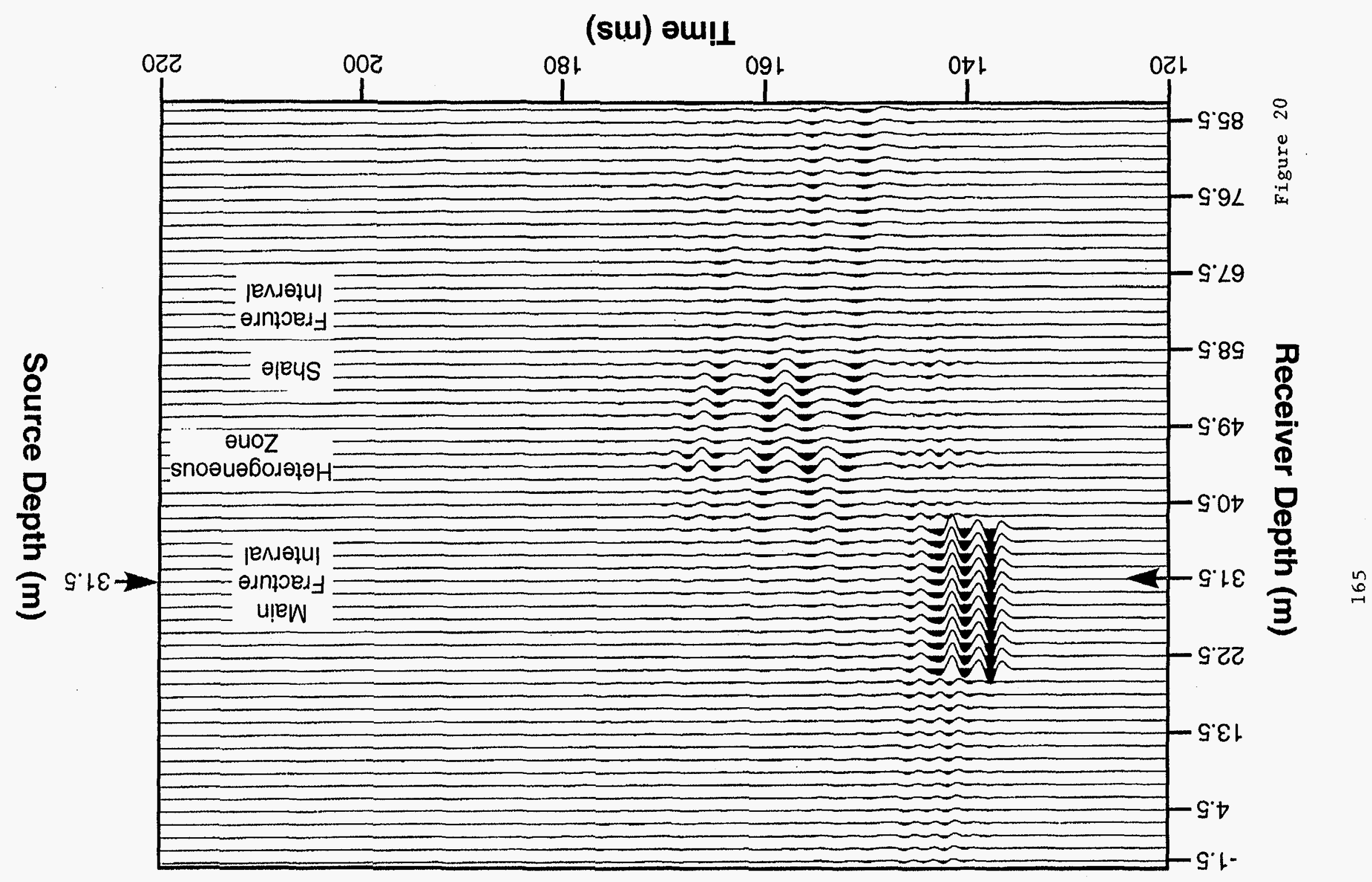

u $008=$ uo!łededəS $\|{ }^{\prime} M$

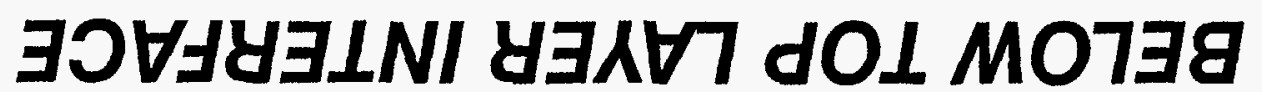

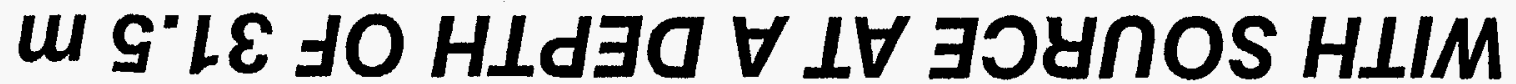

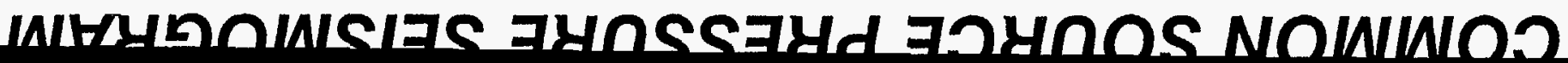


AMPLITUDE-DEPTH DISTRIBUTION OF HORIZONTAL PARTICLE VELOCITY PRODUCED BY A SOURCE AT A DEPTH OF $31.5 \mathrm{~m}$ BELOW TOP LAYER INTERFACE

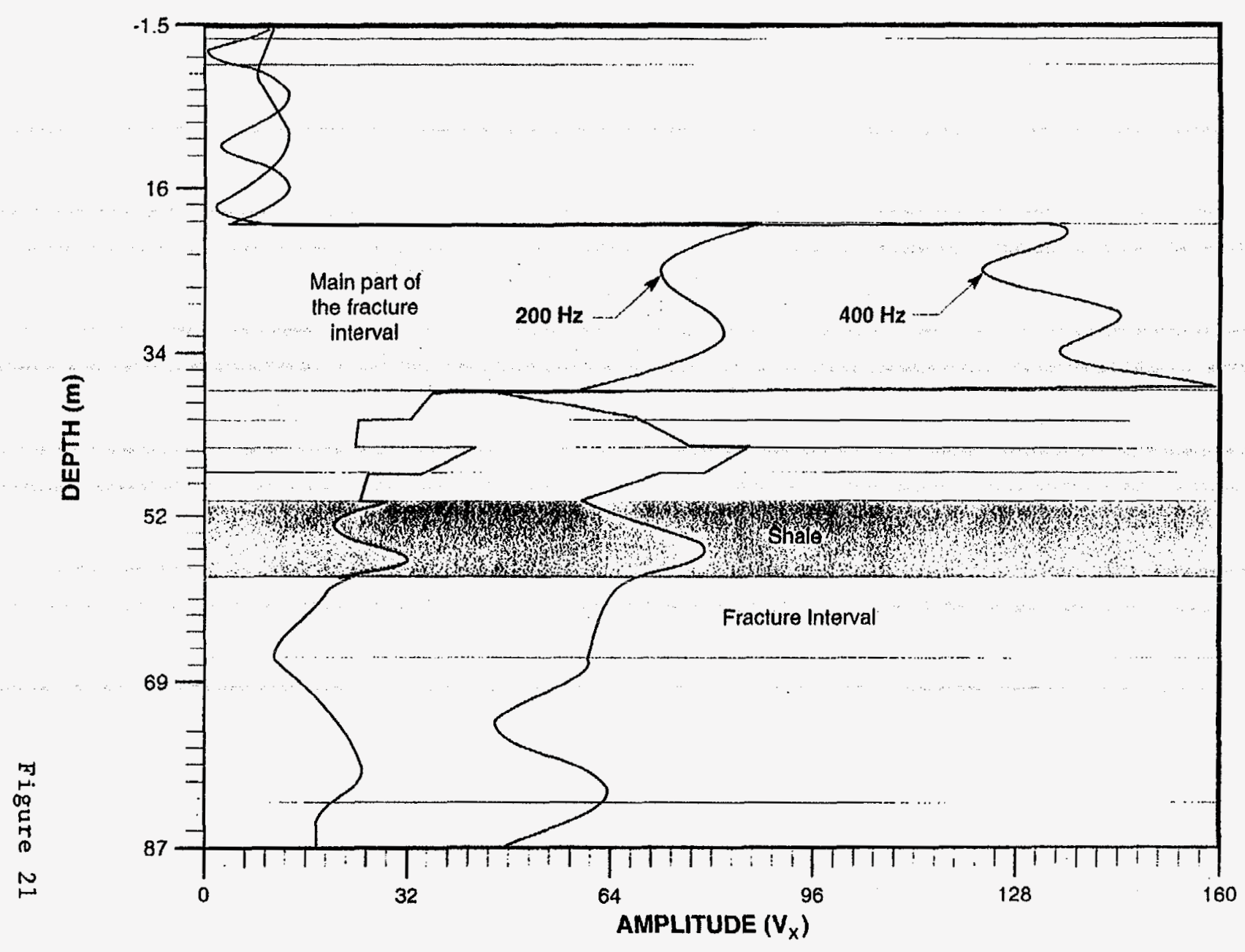


COMPARISON OF SPECTRAL DENSITY OF

HORIZONTAL \& VERTICAL PARTICLE VELOCITY WITH SEISMIC NOISE LEVELS IN NOISY \& QUIET ENVIRONMENTS

SOURCE DEPTH $=31.5 \mathrm{~m}$ BELOW TOP LAYER INTERFACE

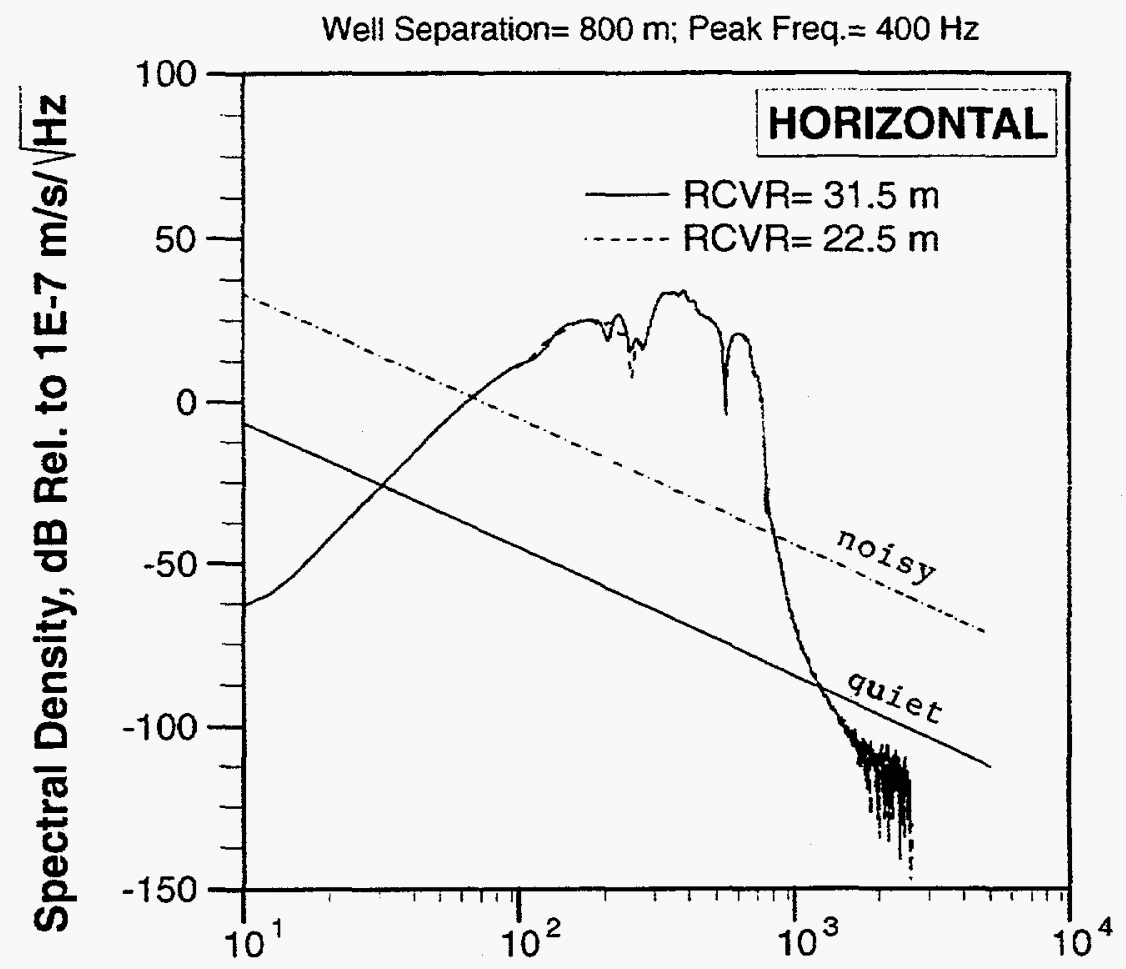

(a)

SOURCE DEPTH $=31.5 \mathrm{~m}$ BELOW TOP LAYER INTERFACE

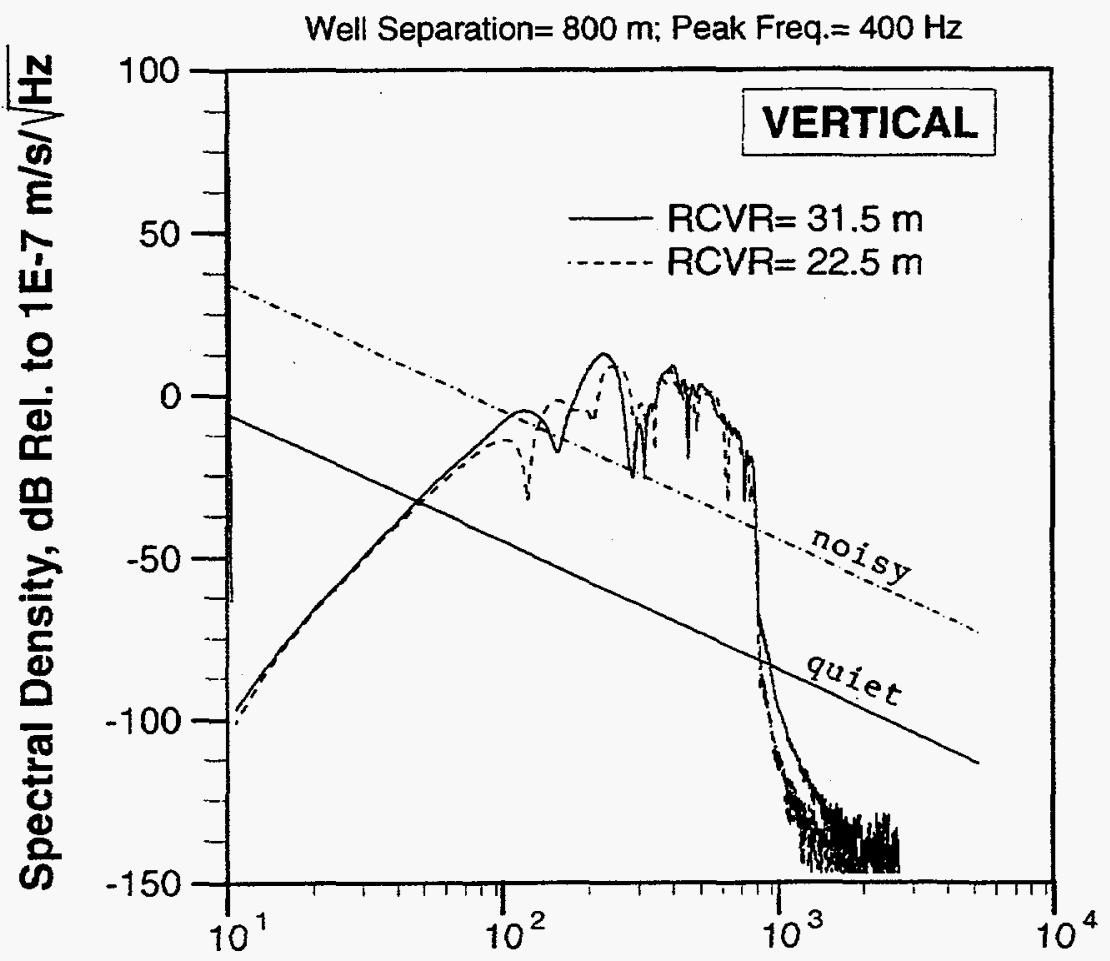

(b) Frequency $(\mathrm{Hz})$ 


\section{COMPARISON OF SPECTRAL DENSITY OF PRESSURE WITH ELECTRONIC SEISMIC NOISE LEVELS IN QUIET ENVIRONMENTS}

SOURCE DEPTH $=31.5 \mathrm{~m}$ BELOW TOP LAYER INTERFACE

Well Separation $=800 \mathrm{~m}$; Peak Freq $=400 \mathrm{~Hz}$

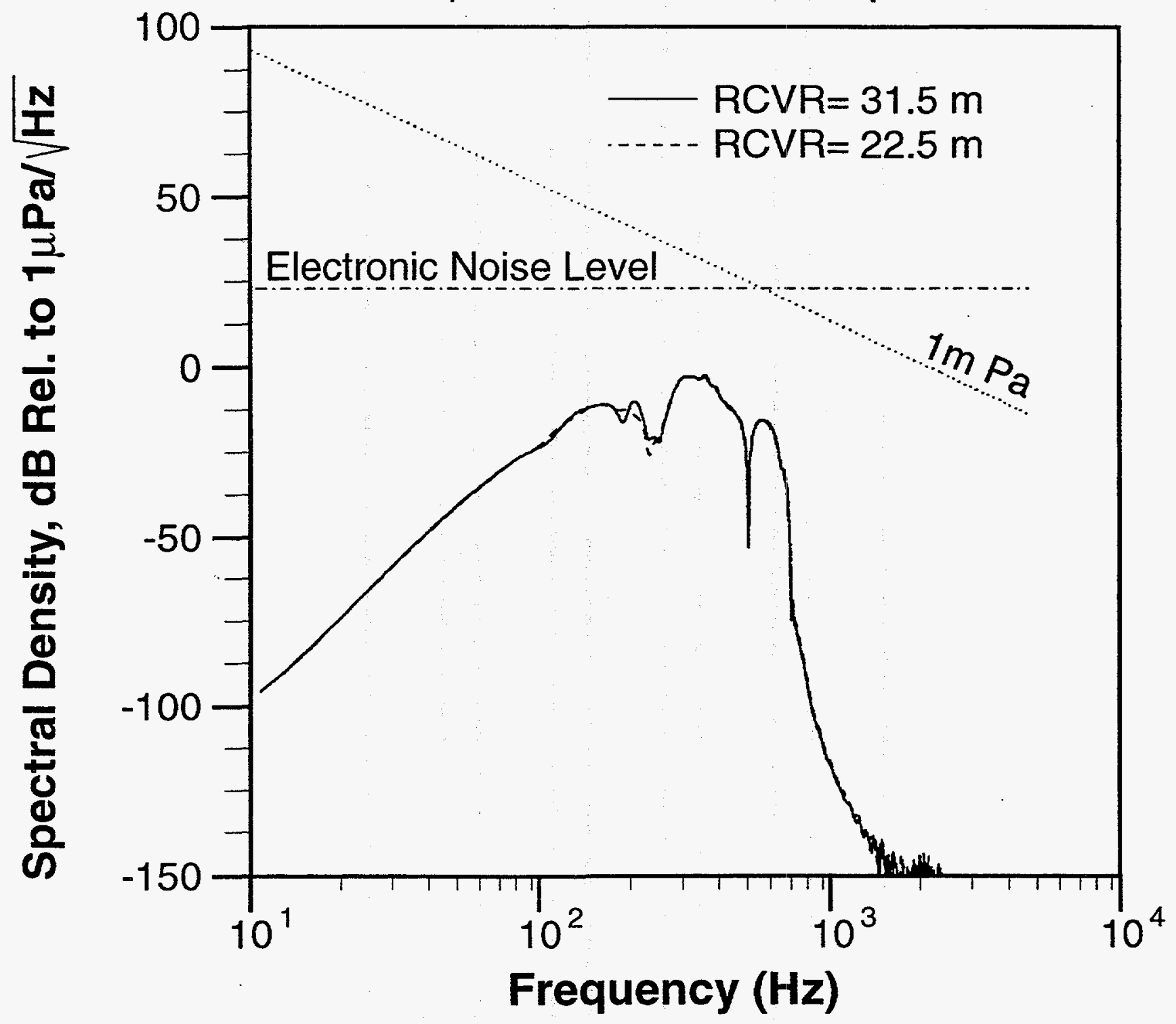

Figure 23 


\section{COMMON SOURCE SEISMOGRAMS WITH SOURCE AT A DEPTH OF $55 \mathrm{~m}$ BELOW TOP LAYER INTERFACE}

Well Separation $=800 \mathrm{~m}$

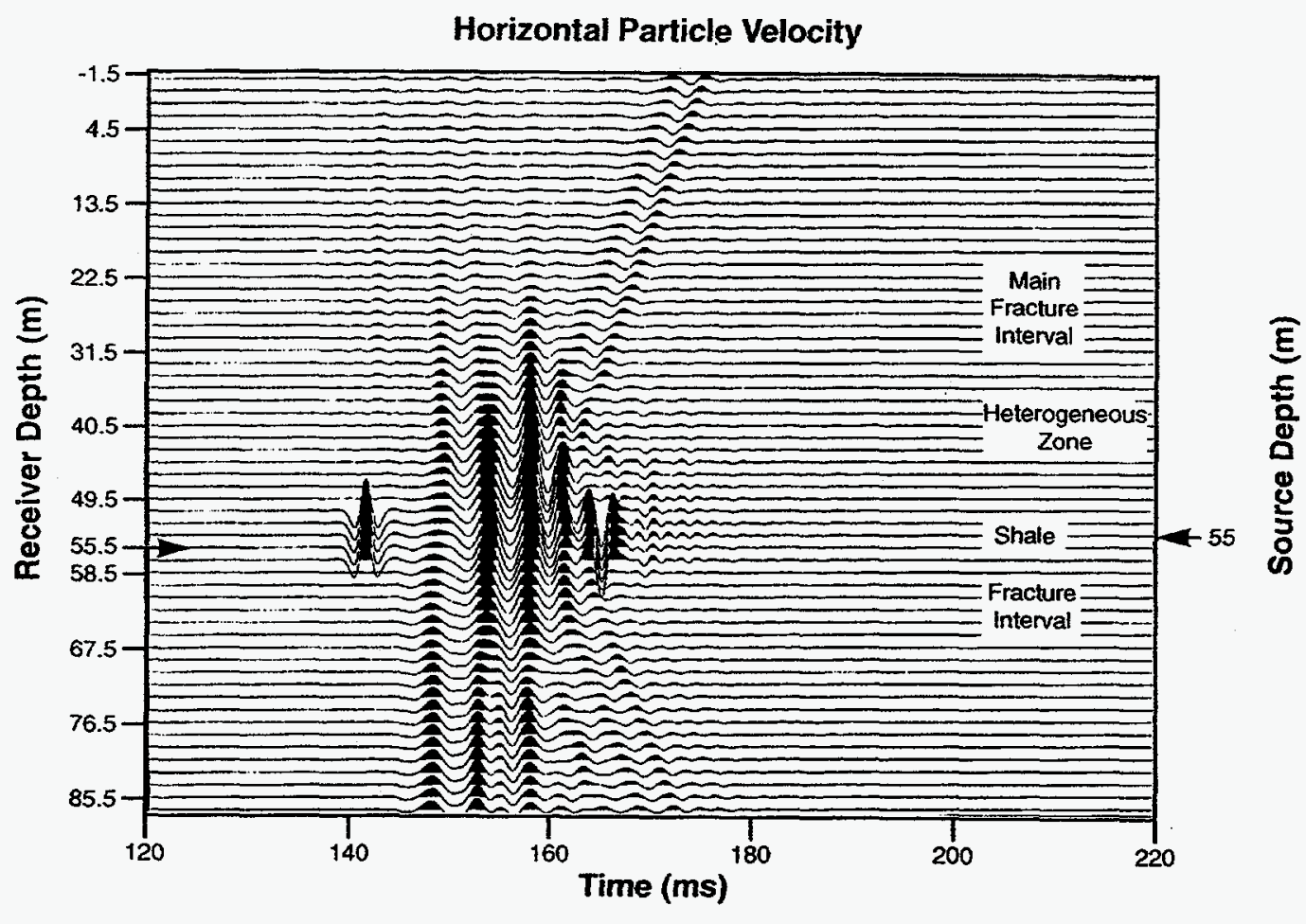

(a)

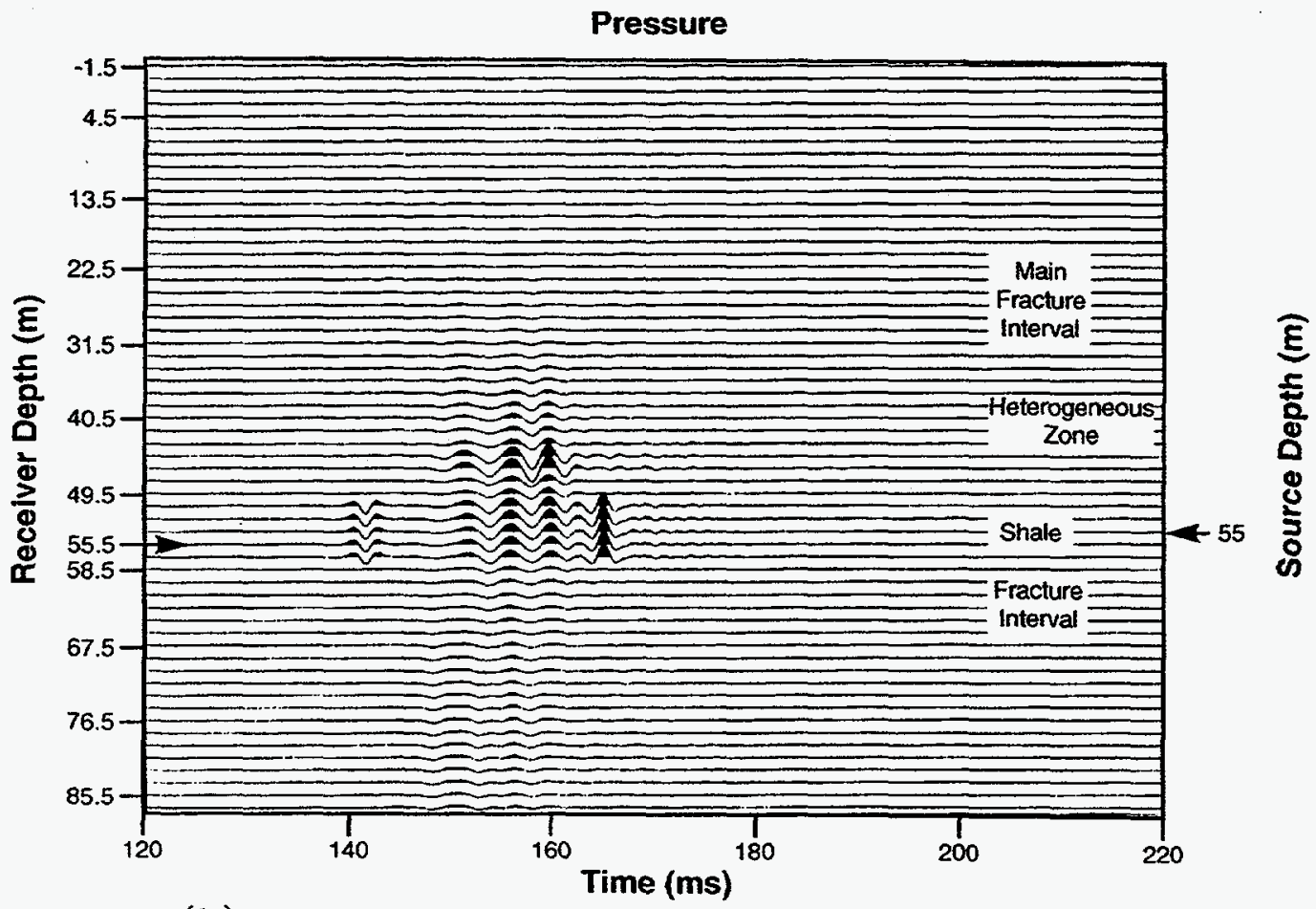

(b)

Figure 24 


\section{COMMON SOURCE VERTICAL VELOCITY SEISMOGRAMS WITH}

SOURCE AT A DEPTH OF $55 \mathrm{~m}$ BELOW TOP LAYER INTERFACE

Well Separation $=800 \mathrm{~m}$
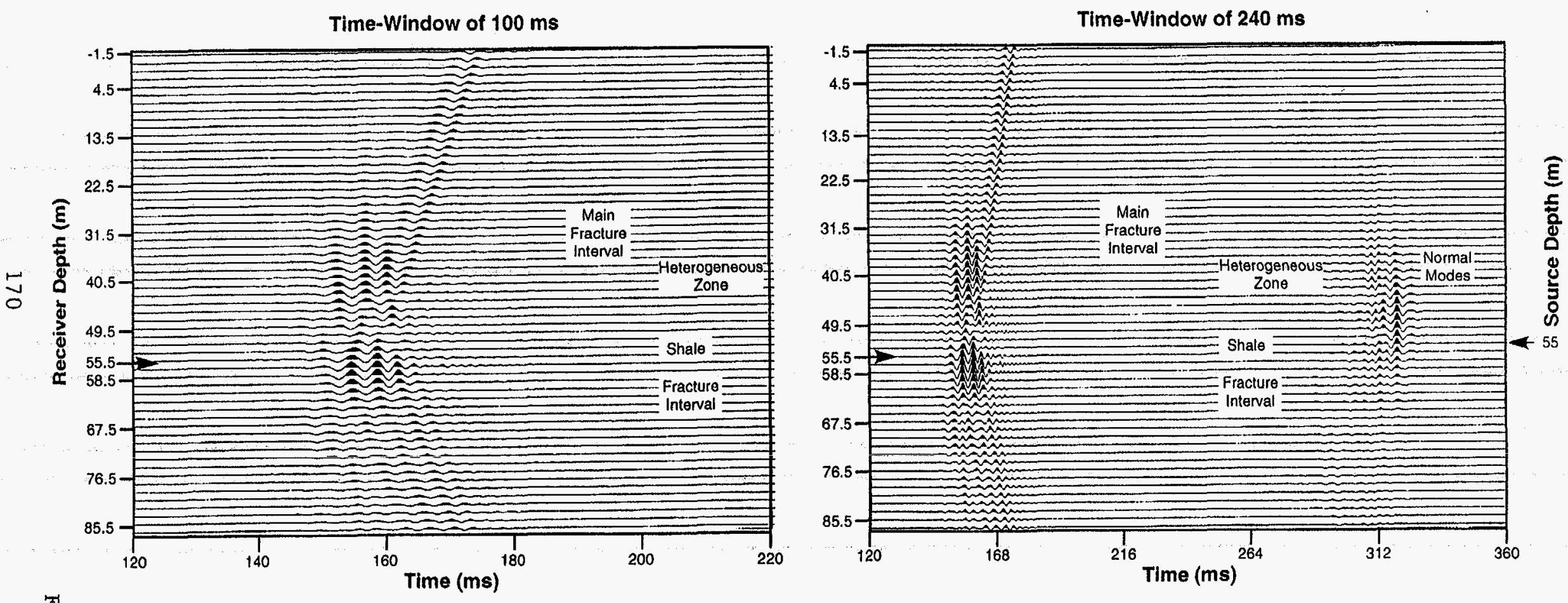

(a)

(b) 


\section{AMPLITUDE-DEPTH DISTRIBUTION OF VERTICAL PARTICLE VELOCITY PRODUCED BY A SOURCE AT A DEPTH OF $55 \mathrm{~m}$ BELOW TOP LAYER INTERFACE}

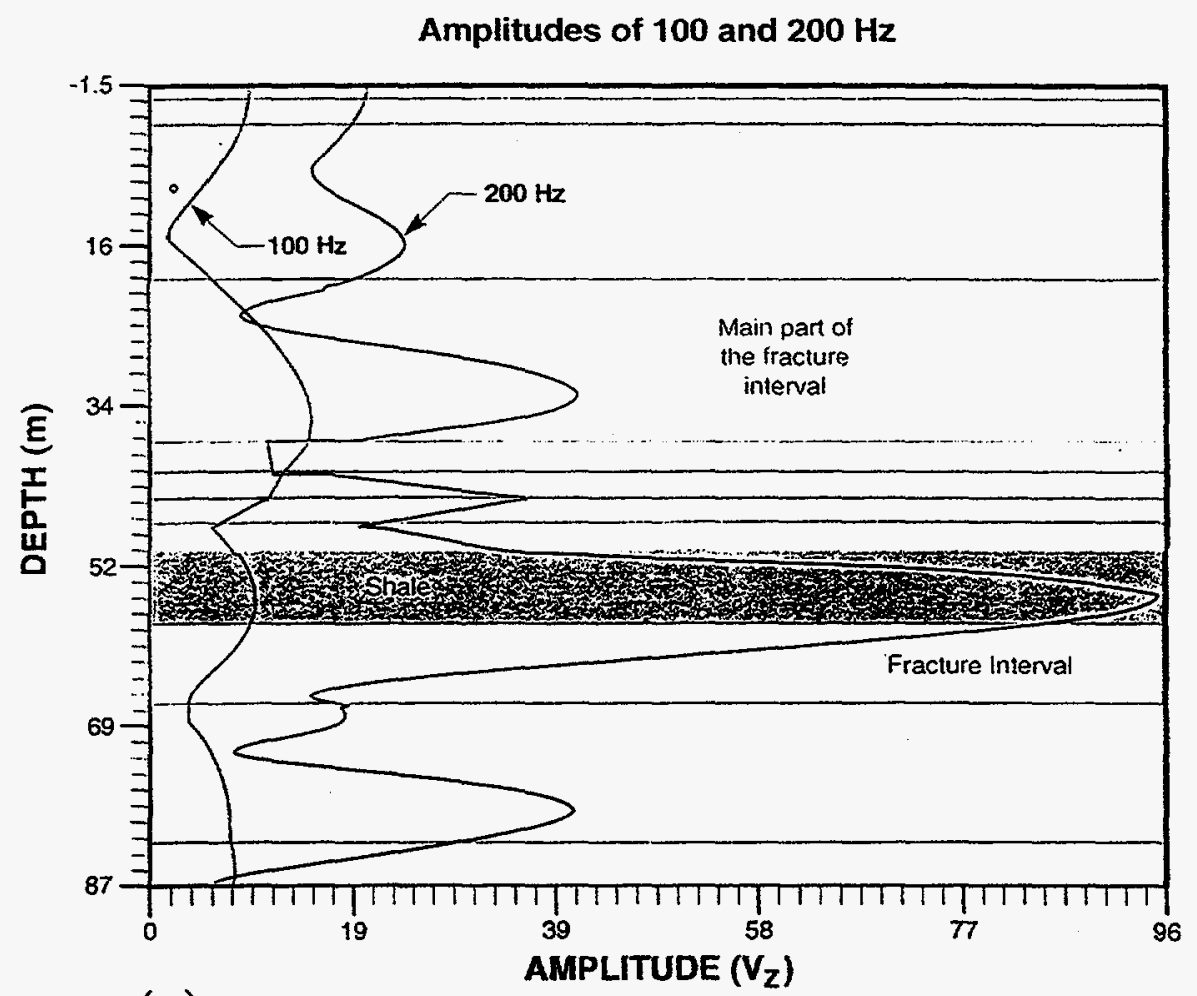

(a)

Amplitudes of 200 and $400 \mathrm{~Hz}$

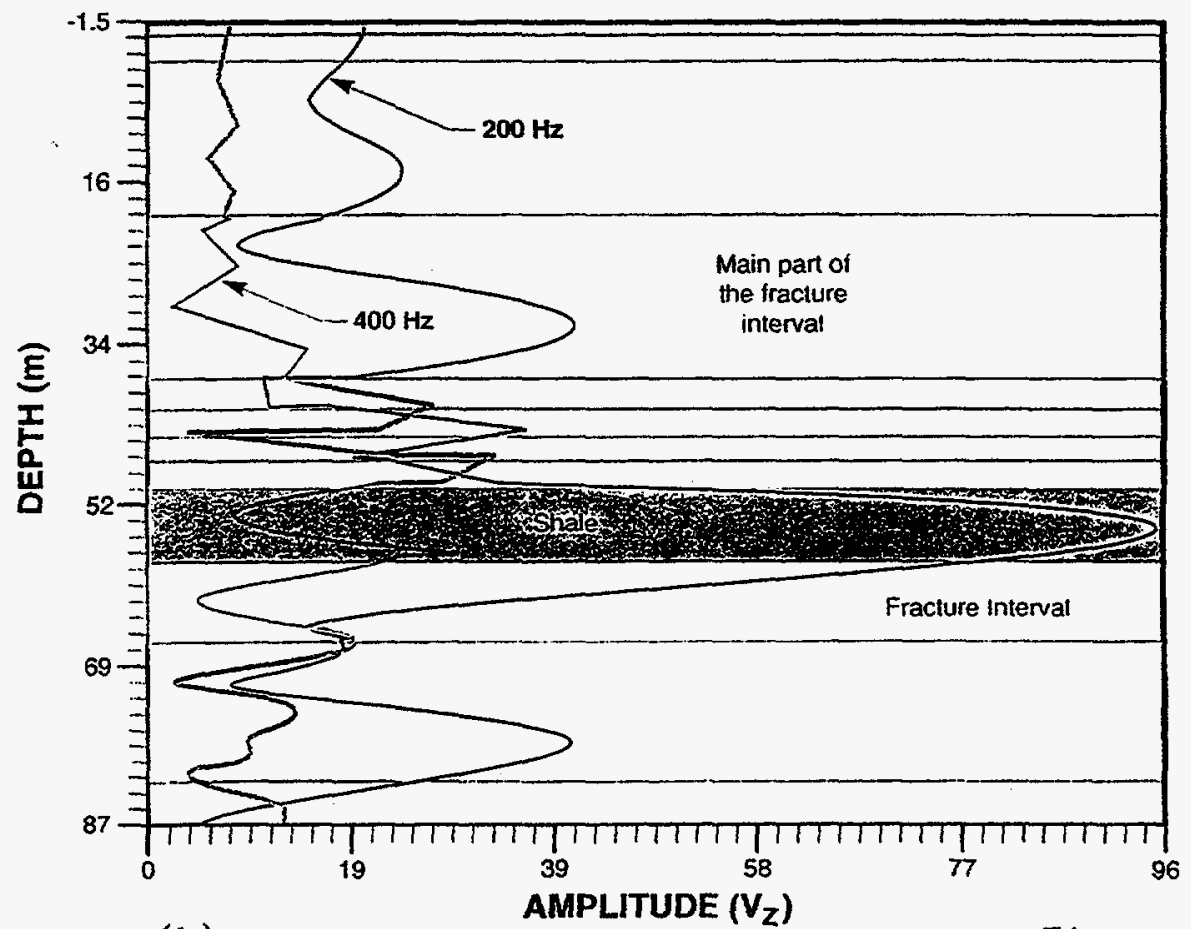

(b)

Figure 26 


\section{COMPARISON OF SPECTRAL DENSITY OF HORIZONTAL \& VERTICAL PARTICLE VELOCITY WITH SEISMIC NOISE LEVELS IN NOISY \& QUIET ENVIRONMENTS}

SOURCE DEPTH $=55 \mathrm{~m}$ BELOW TOP LAYER INTERFACE

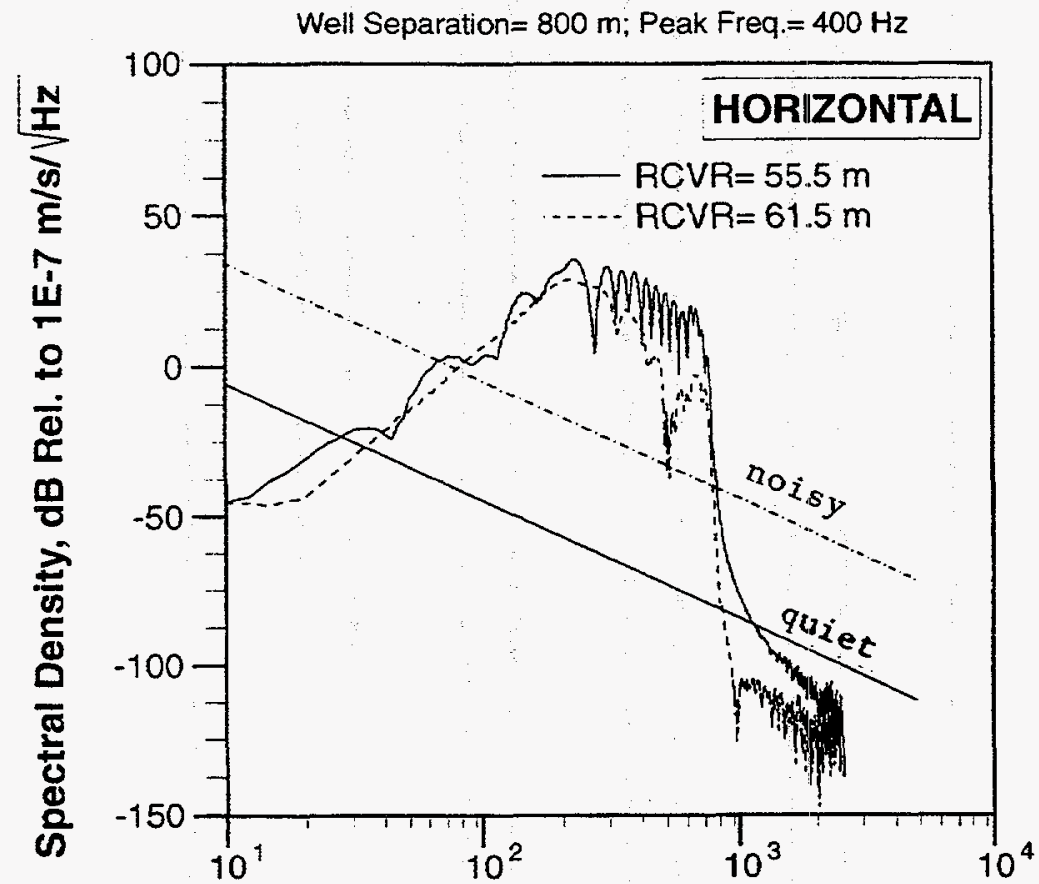

(a)

SOURCE DEPTH $=55 \mathrm{~m}$ BELOW TOP LAYER INTERFACE

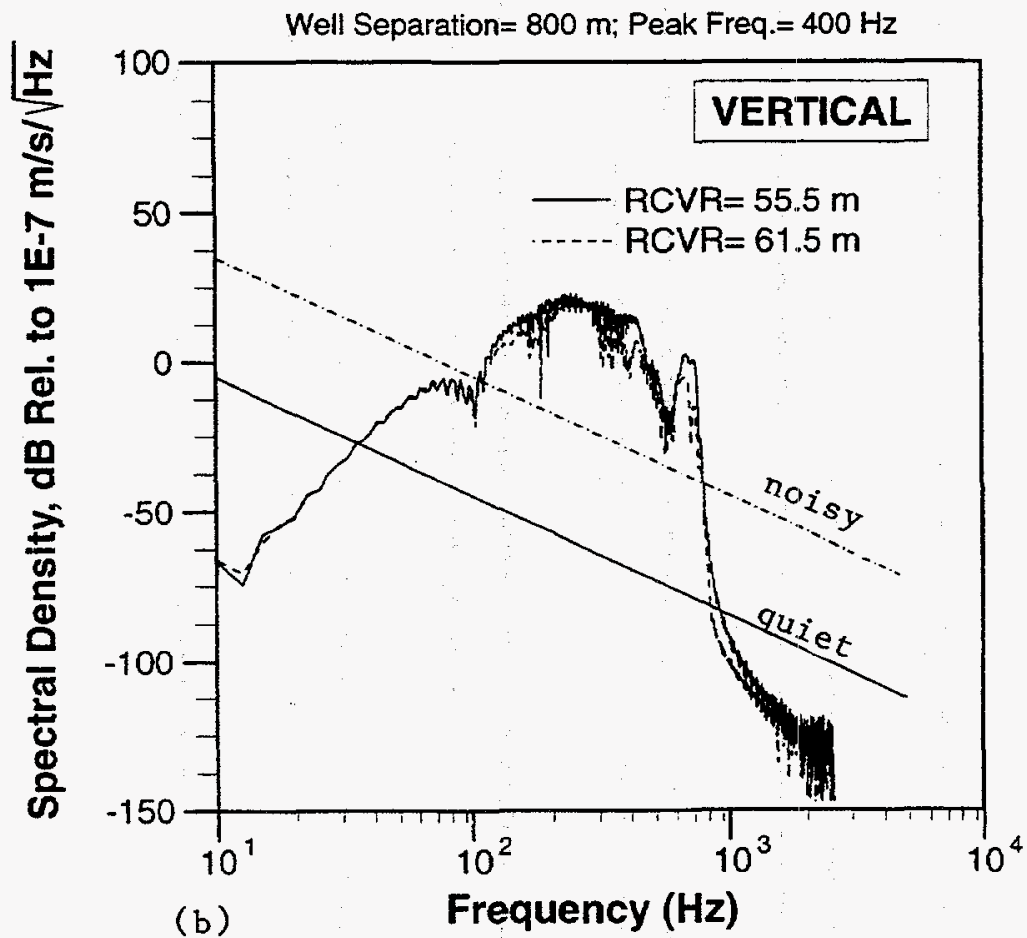




\section{COMPARISON OF SPECTRAL DENSITY OF PRESSURE WITH ELECTRONIC AND SEISMIC NOISE LEVELS IN QUIET ENVIRONMENTS}

SOURCE DEPTH $=55 \mathrm{~m}$ BELOW TOP LAYER INTERFACE

|c

Well Separation $=800 \mathrm{~m}$; Peak Freq. $=400 \mathrm{~Hz}$

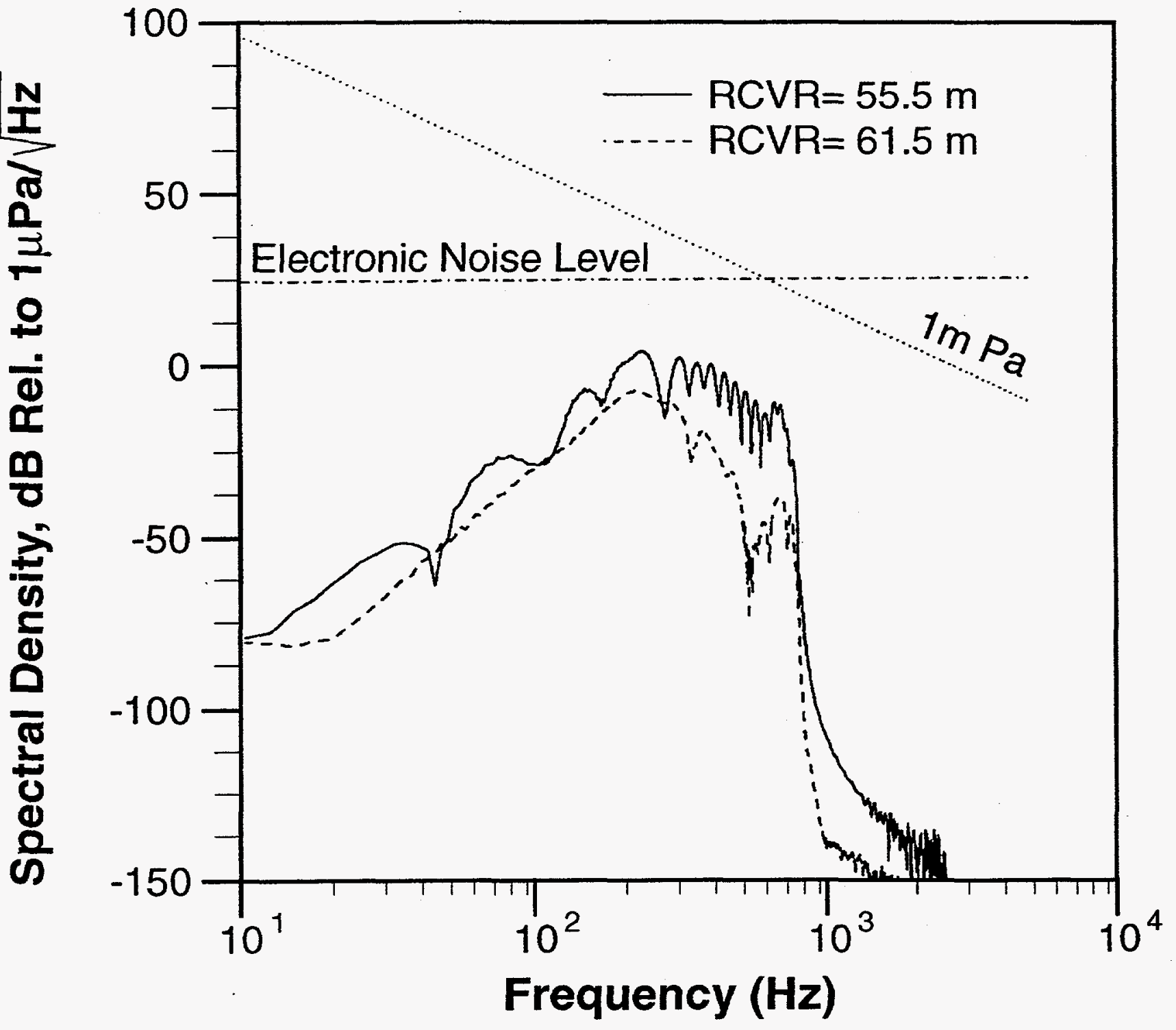

Figure 28 


\section{TECHNOLOGY TRANSFER}

\section{A. Technology Transfer Topics - Phase I}

Four technical research topics were studied in detail during the past year (Phase I) of the project. The work accomplished in these studies provides the basis for focused transfer of project milestone progress information on characterization of fractured reservoirs directly to the oil and gas industry. These accomplishments are also being widely disseminated to industry and to the scientific community through technical presentations at conferences and in peer-review publications. The four research topics are:

(1) Theoretical analysis of seismic wave propagation in poroelastic anisotropic formations, incorporating Biot and squirt-flow fluid permeability petrophysical characteristics in the medium. This analysis is based on plane harmonic compressional and shear waves propagating in a horizontally layered medium. Parametric studies are carried out using the derived theoretical models to determine the seismic dispersion and attenuation effects of permeability anisotropy.

(2) Theoretical analysis of monopole and horizontal dipole source logging tool responses (acoustic pressure waves and elastic wave displacements) in a poroelastic anisotropic formation. Parametric studies of fullwave sonic logs are carried out to indicate and aid in the interpretation of formation permeability anisotropy.

(3) Application of the above theoretical seismic and sonic log response models to geologic and petrophysical conditions in the Union Pacific Resources Company Twin Creek reservoir (a fractured reservoir in the Utah-Wyoming Overthrust Belt).

(4) Semi-analytical model of multiphase fluid flow in fractured rock reservoirs. Simulation of 3D multiphase flow distributions using the approximate 3D geometry of the Twin Creek fractured reservoir.

\section{B. Industry Contacts and Application Interests}

A formal letter of invitation was sent to reservoir geophysicists and engineers in the oil and gas industry and to scientists and engineers in the oil field services industry. This letter described the four primary projects being studied and announced plans for organizing and conducting technical workshops on the research project activities in the second year (Phase II) and on added topics in the third year (Phase III). More than 100 geologists, geophysicists, and engineers were invited to respond by indicating their interest in: (1) receiving project milestone technical newsletters; (2) participating in future project-oriented workshop activities; and (3) denoting their specific technical application areas of interest in reservation characterization. The letter was very successful in establishing contact with 62 professional individuals representing 30 oil and gas companies and their divisions. All of the responding individuals expressed strong interest in the project goals and projected research studies. Nearly all of these respondents also indicated their interest in participating in the technology workshops. Appendix E presents a specimen of the project announcement and letter of invitation sent to industry. 
[NOTE: Appendix E is contained in a separate WP6.1 file: niperann.app]

The oil and gas industry companies and the number of interested individuals in each company responding to the project announcement letter are:

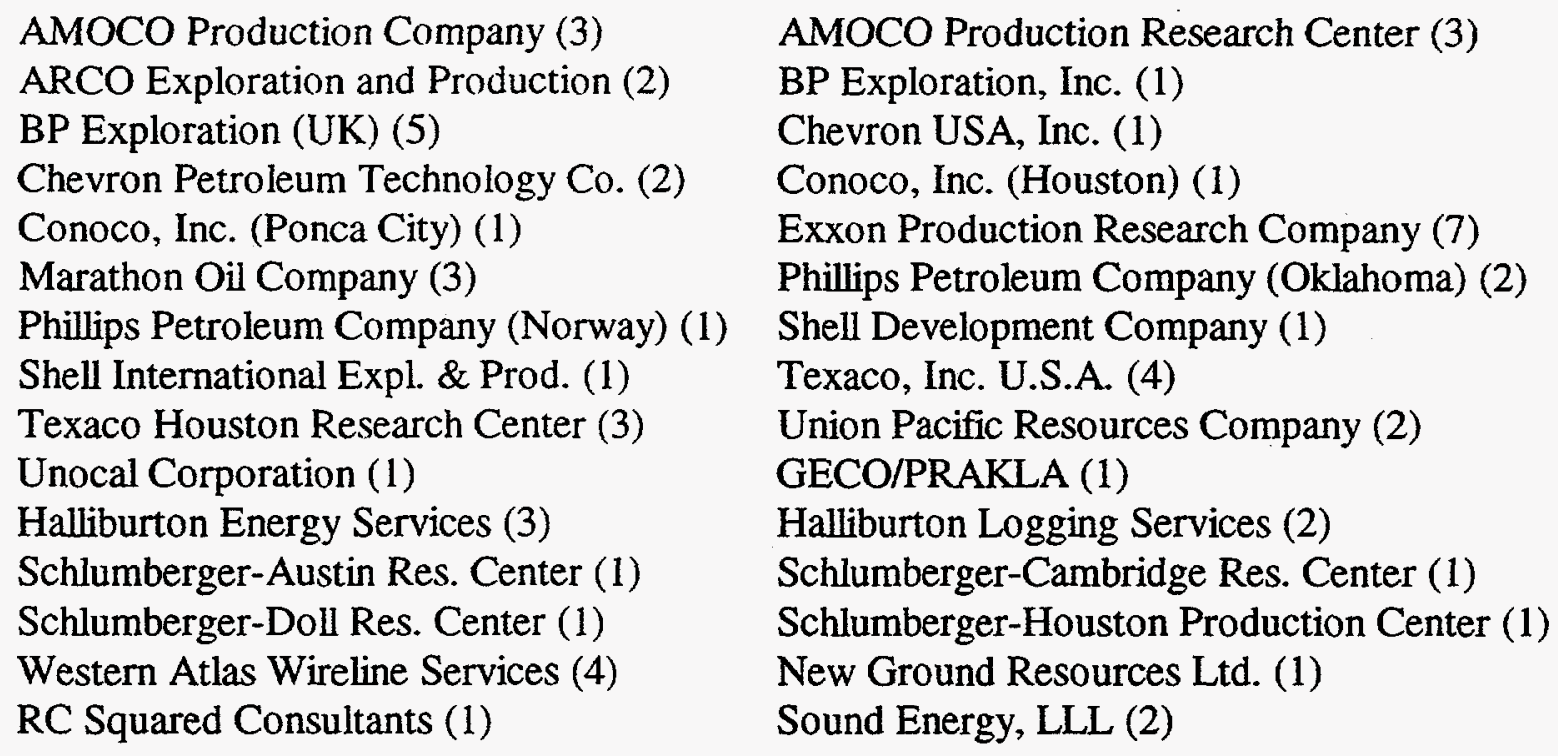

The application areas of interest indicated by the responding industry representatives are summarized as follows:

\begin{tabular}{cc} 
Reservoir Geophysics & \\
\hline Surface: & $75 \%$ \\
Downhole: & $85 \%$ \\
Technology: & $67 \%$
\end{tabular}

Reservoir Engineering

Production: $52 \%$

Development: $48 \%$

Modeling: $69 \%$

$\frac{\text { Other Topics: }}{4 \%}$

\section{Project Newsletter}

Project research studies conducted during the first year (Phase 1) have resulted in three technical papers scheduled to be published in the project newsletter during the first quarter of Phase II. These papers are entitled: 
(1) "The Transversely Isotropic Poroelastic Wave Equation Including the Biot and the Squirt Mechanisms: Theory and Application" by J.O. Parra (accepted for publication in Geophysics, 1997)

(2) "Seismic Signatures of the Lodgepole Fractured Reservoir in the Utah-Wyoming Overthrust Belt" by J.O. Parra, H.A. Collier, and B. Angstman

(3) "Semi-Analytic Approach to Multiphase Flow Computations" by A. Datta-Gupta and S. Peddibhotla

These research papers are the result of project accomplishments achieved during the past year and are considered ready to be communicated to industry for information and review. The essential aspect of this communication is to present, in an objective way, the project research studies at their earliest practical stages for industry peer review and to solicit possible guidance regarding industry requirements relevant to the research topics.

The strategy underlying the project newsletter is to present such technical milestones in summary report form, provide an avenue for industry response to those accomplishments, and, through follow-up contacts with industry, clarify or otherwise incorporate industry concerns into the research program wherever practical. The newsletter will also serve as a vehicle for soliciting broader industry interest and participation by requesting the recipients to recommend other individuals who may be interested in the work and to identify other potential field sites where the fractured reservoir technology might be evaluated. To facilitate this expansion of interest in the project, the newsletter format will include a convenient reader reply form for returning technical review comments and suggestions on the work.

Work anticipated in the second year (Phase II) will include plans for a project-oriented seminar and workshop designed to communicate the accomplishments of Phase I to industry and to host presentations on related topics by industry. The expected results of this mechanism of information exchange will provide timely industry review of the work being accomplished and a clearly defined consensus on the industry priorities for the fractured reservoir characterization technology under study and development. 


\section{APPENDIX E \\ Southwest Research Institute Letterhead and SwRI mailing labels}

ADDRESSEE

September 6, 1996

CCCCCCC Company

Dear MM. MMMMM:

This letter describes an active research project underway at Southwest Research Institute for

CHARACTERIZATION OF FRACTURED RESERVOIRS USING STATIC AND DYNAMIC DATA: FROM SONIC AND 3D-SEISMIC TO PERMEABILITY DISTRIBUTION

sponsored by the National Institute for Petroleum and Energy Research (NIPER), Bartlesville, Oklahoma. Our purpose in contacting you is to inform you of the project goals and activities and to enlist your interest in following the progress of this work and possibly participating in its industryoriented seminars and workshops beginning next Spring. Similar contacts are being made with scientists and engineers at more than 20 oil, gas, and field service companies to develop a strong and broadly based interest group.

Attached is a brief summary of the project describing its primary objectives and the research tasks for Phase I of the three year program. One of our primary goals is to disseminate the project information as it progresses and to transfer its useful results and technology related to characterization of fractured reservoirs to all interested parties. A reply form and return envelope is enclosed by which you can express your scientific and/or engineering interests in the project and be placed on the project information distribution list. Participation bears no obligations whatsoever.

We invite you to sign up to receive our research progress reports and workshop schedule on characterization of fractured reservoirs. If you should not wish to participate, we ask that you please pass this letter and reply form on to a colleague at CCCCCC Company who may be interested.

If there are any questions concerning our present project activities or the planned project seminars and workshops, please contact

Thomas E. Owen, Ph.D., P.E.

Task Leader, Technology Transfer - Proj. 15-17346

Southwest Research Institute

P.O. Box 28510

Ph. (210) 522-2715

San Antonio, TX 78228-0510

Fax (210) 647-4325 
or

Jorge O. Parra, Ph.D.

Project Leader, Proj. 15-17346

Southwest Research Institute

P.O. Box 28510

Ph. (210) 522-3284

San Antonio, TX 78228-0510

Fax (210) 647-4325

We look forward to receiving your reply soon.

Sincerely,

Thomas E. Owen

Task Leader, Technology Transfer

$\mathrm{TEO} / \mathrm{dl}$ 


\title{
CHARACTERIZATION OF FRACTURED RESERVOIRS USING STATIC AND DYNAMIC DATA: FROM SONIC AND 3D-SEISMIC TO PERMEABILITY DISTRIBUTION
}

\author{
A Research Project in Progress \\ Sponsored by \\ National Institute for Petroleum and Energy Research (NIPER) \\ Bartlesville, Oklahoma \\ (Contract No. G4S51731) \\ at \\ Southwest Research Institute \\ San Antonio, Texas \\ (SwRI Project 15-17346)
}

SOLICITING INDUSTRY INTEREST

AND

PARTICIPATION IN FUTURE SEMINARS AND WORKSHOPS 


\section{CHARACTERIZATION OF FRACTURED RESERVOIRS USING STATIC AND DYNAMIC DATA: FROM SONIC AND 3D-SEISMIC TO PERMEABILITY DISTRIBUTION}

A Research Project in Progress

Sponsored by

National Institute for Petroleum and Energy Research (NIPER)

Bartlesville, Oklahoma

(Contract No. G4S51731)

Performed by

Southwest Research Institute

San Antonio, Texas

(SwRI Project 15-17346)

|||||||||||||||||||||||||||||||||||||||||||||||||||||||||||||||||||||||||||||||||||||||||||||||||||||||||||||||||||||||||||||||||||||||||||||||||||||||||||||||-

Project Time Schedule: March 1996 through November 1998 (32 months)

Project Organization:

PHASE I (March 1996 - February 1997)

Theoretical Models Using Deterministic Interwell Seismic Solutions, Petrophysical Analysis, and Semi-Analytical Approaches to Multiphase Flow in Fractured Formations

PHASE II (March 1997 - December 1997)

Theoretical Models Using Stochastic Solutions, Fracture Connectivity Using Production Data, and Interwell Seismic/Sonic Log Signal Analysis

PHASE III (January 1998 - November 1998)

Numerical Modeling, Analysis, and Integration of Geophysical, Petrophysical, and Engineering Data to Characterize Fractured Reservoirs 


\section{PHASE I RESEARCH STUDIES AND INFORMATION DISSEMINATION TOPICS}

(1) Theoretical analysis of seismic wave propagation in poroelastic anisotropic formations, including Biot and squirt-flow fluid permeability petrophysical characteristics in the medium. Plane harmonic compressional and shear waves in a horizontally layered medium having azimuthal anisotropy. Parametric studies using the derived theoretical models to determine the seismic dispersion and attenuation effects caused by permeability anisotropy.

(2) Theoretical analysis of monopole and horizontal dipole source logging tool responses (acoustic pressure waves and elastic wave displacements) in a poroelastic anisotropic medium. Parametric studies of fullwave sonic logs to indicate and aid in the interpretation of formation permeability anisotropy.

(3) Apply theoretical seismic and sonic log response models to geologic and petrophysical conditions in the Union Pacific Resources Company Twin Creek Reservoir (a fractured rock reservoir in the Utah and Wyoming Overthrust).

(4) Semi-analytical model of multiphase fluid flow in fractured rock reservoirs. Simulation of 3D multiphase flow distributions using the approximate 3D geometry of the Twin Creek Reservoir.

KEY PROJECT PERSONNEL

Project Leader:

Signal Processing:

Petrophysics and

Reservoir Geology:

Reservoir Engineering:

Technology Transfer:
Jorge O. Parra, Ph.D.

Principal Scientist, SwRI

Brian J. Zook, Ph.D.

Research Scientist, SwRI

Hughbert A. Collier, Ph.D.

Asst. Prof., Geology

Tarlton State University

(Consultant to SwRI)

Akhil Datta-Gupta, Ph.D.

Asst. Prof., Petroleum Engineering

Texas A\&M University

(Consultant to SwRI)

Thomas E. Owen, Ph.D., P.E.

Institute for Research in Sciences and Engineering

The University of Texas at San Antonio

(Consultant to SwRI) 
RESEARCH PROJECT: Characterization of Fractured Reservoirs Using Static and Dynamic Data: From Sonic and 3D-seismic to Permeability Distribution

1. YES. I am interested in receiving the Project Progress Newsletter on the research project.

Name:

Position:

Company:

Mail Address:

Telephone:

Fax Number:

E-Mail:

2. My technical interests related to the project are (check all topics that apply):

$\begin{array}{cc}\text { Reservoir Geophysics } & \text { Reservoir Engineering } \\ \square \text { Surface } & \square \text { Production } \\ \square \text { Downhole/Interwell } & \square \text { Development } \\ \square \text { Technology } & \square \text { Modeling } \\ \text { Reservoir Geology } & \text { Formation Evaluation } \\ \square \text { Structure } & \square \text { Log Analysis/Fullwave Sonic } \\ \square \text { Petrophysics } & \square \text { Fracture Interpretation }\end{array}$

3. I am interested in the future seminars and workshops on characterization of fractured reservoirs. $\quad \square$ Yes $\square$ No

4. Others in my company who may be interested in this project and its newsletter are:

NAME____ADDRESS (if different from mine)____




\section{CONCLUSIONS AND ACCOMPLISHMENTS}

The basic theoretical analysis in geophysics and petroleum engineering were completed. The geophysical techniques included the theoretical development of plane-harmonic seismic waves in poroelastic and anisotropic layered media, and the theoretical development of a multipole acoustic logging system in a fluid-filled borehole surrounded by an anisotropic poroelastic and anisotropic viscoelastic formation. The petroleum engineering techniques included the development of a semianalytical approach to multiphase flow calculations and applications.

The feasibility of using seismic measurement techniques to map fracture zones in the Twin Creek reservoir (between wells spaced 2,400 feet apart at a depth of 11,000 feet) was completed. To determine the rock physical properties and the reservoir geometries for the computer models, well logs and thin sections of cutting were integrated with 2D seismic sections. The results of the integration delineated the major boundary surfaces between members of the Twin Creek formation. The integration of the surface seismic and well logs demonstrates that the fracture zones cannot be resolved by surface seismic measurements alone. Instead, it is more appropriate to use highresolution cross-well seismic data. The results of the feasibility study suggests that it may be possible to map variations in the geometry of the fracture zone with borehole reflection imaging and traveltime tomography. In addition, the modeling results suggests that the trapped energy and normal modes excited in the low-velocity shales will be useful to determine the degree of continuity of permeability barriers, layer thickness variations, inhomogeneities, and boundary surfaces.

The boundaries delineated with the geophysical and petrophysical data will be used as input parameters together with the Twin Creek production data to test the multiphase flow model based on the semi-analytical approach.

The manuscript titled "The transversely isotropic poroelastic wave equation: theory and application," was published in the January-February issues of Geophysics, Vol. 62, p. 309-318.

The poster paper titled "Seismic signatures of the fractured Twin Creek Reservoir in Lodgepole field, Utah-Wyoming overthrust belt," was presented at the $4^{\text {th }}$ International Characterization Technical Conference, Advances in Reservoir Characterization for Effective Reservoir Management. This conference was co-sponsored by the U.S. Department of Energy and BDM-Oklahoma, Inc.

A manuscript titled "The analysis and seismic responses of wave propagation in poroelastic media having azimuthal anisotropy" (given in this report) will be submitted to Geophysics in the first quarter of the second year project efforts.

Acoustic logging responses for a multipole source in a fluid-filled borehole surrounded by an anisotropic viscoelastic medium will be calculated in the second year project. The theory and application will be submitted for publication in a peer review journal. 
Seismic responses will be calculated for plane-harmonic waves propagating in poroelastic and anisotropic layered media. This theory and application will be submitted for publication in a peer review journal.

The manuscript titled, "Rapid simulation of multiphase flow through fine-scale geostatistical realizations using a new 3D streamline model: A field example," was published in the Proceedings of the $11^{\text {th }}$ SPE Petroleum Computer Conference held in Dallas, Texas, June 1996.

The manuscript titled "Multiphase streamline modeling in three dimensions: Further generalizations and a large-scale field example," was published in the Proceedings of the $14^{\text {th }}$ SPE Reservoir Simulation Symposium held in Dallas, Texas, June 1997. 